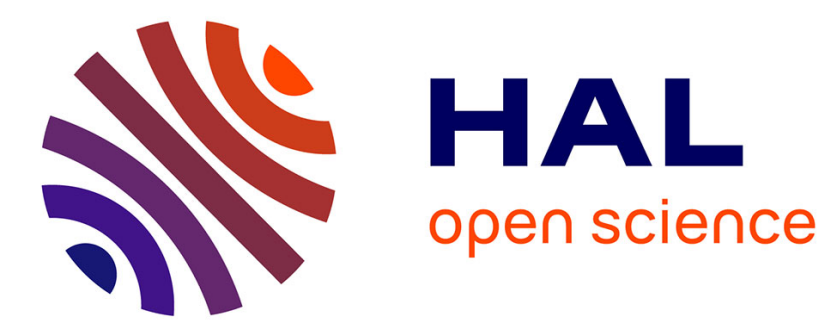

\title{
Fonction zêta des hauteurs des variétés toriques non déployées
}

David Bourqui

\section{To cite this version:}

David Bourqui. Fonction zêta des hauteurs des variétés toriques non déployées. American Mathematical Society, pp.vii-151, 2011, Memoirs of the American Mathematical Society volume $211 \mathrm{n}^{\circ} 994$, 978-0-8218-4936-1. 10.1090/S0065-9266-2010-00609-4 . hal-00004037v2

\section{HAL Id: hal-00004037 https://hal.science/hal-00004037v2}

Submitted on 17 Jul 2007

HAL is a multi-disciplinary open access archive for the deposit and dissemination of scientific research documents, whether they are published or not. The documents may come from teaching and research institutions in France or abroad, or from public or private research centers.
L'archive ouverte pluridisciplinaire HAL, est destinée au dépôt et à la diffusion de documents scientifiques de niveau recherche, publiés ou non, émanant des établissements d'enseignement et de recherche français ou étrangers, des laboratoires publics ou privés. 


\title{
Fonction zêta des hauteurs des variétés toriques non déployées \\ Height zeta functions of nonsplit toric varieties
}

\author{
David BOURQUI
}

\begin{abstract}
We investigate the anticanonical height zeta function of a (non necessarily split) toric variety defined over a global field of positive characteristic, drawing our inspiration from the method used by Batyrev and Tschinkel to deal with the analogous problem over a number field. By the way, we give a detailed account of their method.

Résumé : Nous étudions la fonction zêta des hauteurs anticanonique d'une variété torique (non nécessairement déployée) définie sur un corps global de caractéristique positive. Nous nous inspirons pour cela de la méthode utilisée par Batyrev et Tschinkel pour traiter la situation analogue en caractéristique zéro, méthode que nous rappelons d'ailleurs en détail.
\end{abstract}

AMS Classification : 11G35, 11G50, 14M25, 11M41

\section{Table des matières}

1 Introduction 4

1.1 Position et origine du problème . . . . . . . . . . . . . . . 4

1.2 L'adaptation de la méthode de Batyrev et Tschinkel en caractéristique positive . . . . . . . . . . . . 8

2 Rappels et notations $\quad 11$

2.1 Quelques notations ................... 11

2.2 Rappels sur les corps globaux . . . . . . . . . . . . . . 12

2.3 Hauteurs d'Arakelov, mesure de Tamagawa et constante de Peyre ....................... 16

3 Tores algébriques 20

3.1 Quelques rappels ... . . . . . . . . . . . . 20

3.2 L'espace adélique associé à un tore algébrique . . . . . . . . . 22 


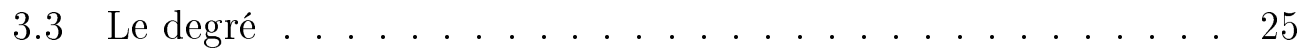

3.3.1 Définitions . . . . . . . . . . . . . . . . . . . 25

3.3.2 Propriétés du degré local dans le cas d'une place finie 32

3.3.3 Propriétés du degré dans le cas arithmétique . . . . . . 32

3.3.4 Propriétés du degré dans le cas fonctionnel . . . . . . . 34

3.4 Groupe de classes . . . . . . . . . . . . . . . . . . . . . . . . . 37

3.5 La dualité de Nakayama . . . . . . . . . . . . . . . . . . 38

3.5.1 Les groupes de cohomologie à la Tate . . . . . . . . . 38

3.5.2 Énoncé de la dualité de Nakayama . . . . . . . . . . 40

3.6 Cocompacité . . . . . . . . . . . . . . . . . . . 40

3.7 Résultats locaux . . . . . . . . . . . . . . . . . . 43

3.8 Résolution flasque d'un tore algébrique et applications _ . . . 46

3.8.1 Rappels et notations . . . . . . . . . . . . . . 46

3.8 .2 Un résultat local . . . . . . . . . . . . . . . . . . . . 47

3.8.3 Approximation faible . . . . . . . . . . . . . . 48

3.8.4 Un invariant des tores algébriques définis sur les corps de fonctions . . . . . . . . . . . . . 53

3.9 Mesure adélique et nombre de Tamagawa d'un tore algébrique 58

4 Hauteurs sur une variété torique et fonction zêta associée 60

4.1 Géométrie des variétés toriques . . . . . . . . . . . . 60

4.1.1 Variétés toriques déployées . . . . . . . . . . . . 6 60

4.1.2 Variétés toriques non déployées . . . . . . . . . . . . 62

4.2 Hauteurs sur une variété torique . . . . . . . . . . . . . . 64

4.2 .1 Hauteurs locales . . . . . . . . . . . . . . . . . . 64

4.2.2 Remarques sur le cas fonctionnel . . . . . . . . . . 69

4.2.3 Hauteurs globales et fonction zêta des hauteurs . . . . 71

4.3 Mesure et nombre de Tamagawa d'une variété torique . . . . . 71

4.4 Le résultat . . . . . . . . . . . . . . . . . . . . 75

4.5 Stratégie de Batyrev et Tschinkel . . . . . . . . . . . . 76

4.5.1 Un peu d'analyse harmonique . . . . . . . . . . . 76

4.5.2 Application à la fonction zêta des hauteurs . . . . . . . 78

5 Calcul des transformées de Fourier et expression intégrale de la fonction zêta des hauteurs $\quad 80$

5.1 Caractères du groupe des idèles . . . . . . . . . . . . . . 80

5.2 Caractères de $T\left(\mathbf{A}_{K}\right) \ldots \ldots \ldots \ldots$. . . . . . . . 81

5.2 .1 Cas arithmétique . . . . . . . . . . . . . . 81

5.2 .2 Cas fonctionnel . . . . . . . . . . . . . . . . 82

5.3 Préliminaires au calcul des transformées de Fourier . . . . . 84

5.4 Les transformées de Fourier locales . . . . . . . . . . . 86 
5.4.1 Cas d'une place finie quelconque . . . . . . . . . 86

5.4 .2 Cas arithmétique . . . . . . . . . . . . 88

5.4 .3 Cas fonctionnel . . . . . . . . . . . . . 95

5.4.4 Cas des places non ramifiées . . . . . . . . . . . 101

5.5 Propriétés analytiques de la transformée de Fourier globale . . 103

5.5.1 Cas arithmétique . . . . . . . . . . 105

5.5.2 Cas fonctionnel . . . . . . . . . . . . 106

5.6 L'expression intégrale de la fonction zêta des hauteurs . . . . . 109

5.6.1 Cas arithmétique . . . . . . . . . . . 110

5.6.2 Cas fonctionnel . . . . . . . . . . . . . 121

6 Évaluation de l'intégrale : le cas arithmétique 127

7 Évaluation de l'intégrale : le cas fonctionnel 129

7.1 Définition d'une certaine classe de fonctions . . . . . . . . . 129

7.2 Un premier exemple . . . . . . . . . . . . . . . . . 130

7.3 Encore quelques définitions . . . . . . . . . . . . . . . . . . 132

7.4 Avertissement au lecteur . . . . . . . . . . . . . . . . 133

7.5 Un lemme de décomposition . . . . . . . . . . . . . . . . . . . . 134

7.5.1 Version simple................. 134

7.5.2 Version générale . . . . . . . . . . . . . 137

7.6 Comportement des fonctions étudiées par intégration . . . . . 143

7.6.1 Le lemme technique : forme dépouillée . . . . . . . . . 144

7.6.2 Le lemme technique : forme simple . . . . . . . . . . 144

7.6.3 Le lemme technique : forme générale . . . . . . . . 146

8 Application aux fonctions zêta des hauteurs 152

8.1 Préliminaires . . . . . . . . . . . . . . . . . . . . . 152

8.2 Application du lemme technique dans le cas arithmétique . . . 153

8.3 Application du lemme technique dans le cas fonctionnel . . . . 154

8.3.1 Le cas d'une extension de déploiement non ramifiée . . 154

8.3.2 Un cas plus général . . . . . . . . . . . . . . . 158

8.4 Calcul du terme principal de la fonction zêta des hauteurs . . 163

8.4.1 Calcul de $C_{0} \ldots \ldots \ldots \ldots$. . . . . . . . 163

8.4 .2 Cas arithmétique . . . . . . . . . . . 167

8.4.3 Cas fonctionnel . . . . . . . . . . . . 167

A Appendice : le cas où l'hypothèse ?? n'est pas vérifiée 168 


\section{Introduction}

\subsection{Position et origine du problème}

Soit $V$ une variété projective définie sur un corps global $K$, i.e. un corps de nombres ou le corps de fonctions d'une courbe projective, lisse et géométriquement intègre, définie sur un corps fini. Soit $H$ une hauteur exponentielle relative à un fibré en droites ample. Alors pour tout réel $B$ le nombre

$$
n_{V, H}(B)=\#\{x \in V(K), H(x) \leqslant B\}
$$

est fini. Si l'ensemble $V(K)$ est dense pour la topologie de Zariski, la quantité $n_{V, H}(B)$ tend donc vers l'infini quand $B$ tend vers l'infini. Une question naturelle est alors d'essayer de décrire le comportement asymptotique de la quantité $n_{V, H}(B)$, en d'autres termes le comportement asymptotique du nombre de points de hauteur bornée. On cherche notamment à interpréter cette description en termes de la géométrie de la variété $V$. C'est l'objet d'un programme initié par Manin et ses collaborateurs, qui s'est révélé extrêmement riche et ouvert : pour la vérification des prédictions de Manin pour des classes particulières de variétés, des techniques très diverses ont pu être employées. Ces prédictions (raffinées par Peyre puis Batyrev et Tschinkel) sont maintenant établies pour plusieurs classes de variétés. Nous renvoyons le lecteur aux textes [Pe5] et [Pe6] pour un état général de la question aux alentours de 2003 et les références de nombreux travaux sur le sujet. On pourra également consulter [Bro] pour un état des lieux récent concernant le cas des surfaces.

Soulignons que la très grande majorité de ces travaux se placent dans le cas où $K$ est un corps de nombres. Ici nous nous intéressons au cas où $K$ est de caractéristique non nulle, cas encore peu exploré dans la littérature. Avant toute chose, nous allons préciser l'une des prédictions de Manin concernant le comportement asymptotique de $n_{V, H}(B)$, dans le cas où le corps de base est un corps de nombres. Elle peut s'énoncer de la manière suivante.

\section{Question 1.1}

Soit $V$ une variété projective et lisse définie sur un corps de nombres $K$. On suppose que la classe du faisceau anticanonique est à l'intérieur du cône effectif, et que l'ensemble $V(K)$ des points rationnels de $V$ est dense pour la topologie de Zariski. Soit $t$ le rang du groupe de Néron-Séveri de V. Soit $H$ une hauteur relative au faisceau anticanonique. Existe-t-il un ouvert de Zariski non vide $U$ de $V$ et une constante $C>0$ tels qu'on ait

$$
n_{U, H}(B) \underset{B \rightarrow+\infty}{\sim} C B \log (B)^{t-1} \quad ?
$$


La restriction à un ouvert $U$ éventuellement strict de $V$ est nécessaire en raison de l'existence possible de fermés acccumulateurs, dont un prototype est donné par les diviseurs exceptionnels sur les surfaces de del Pezzo.

Soulignons que bien qu'il ait été démontré que la question 1.1 avait une réponse positive pour de large classes de variétés, un contre-exemple dû à Batyrev et Tschinkel montre que la réponse à cette question est négative en général (le contre-exemple porte sur la puissance du logarithme apparaissant dans la formule (1.2), cf. [BaTs3]).

Il existe une version fonctionnelle immédiate de la question 1.1 : il suffit de remplacer dans l'énoncé l'hypothèse «K est un corps de nombres» par $\ll K$ est un corps global de caractéristique positive». Cependant, la nature «dispersée» de l'ensemble des valeurs prises par les fonctions hauteurs dans le cas fonctionnel entraîne qu'une formule du type (1.2) ne pourra jamais être vérifiée. Plus précisément, cet ensemble de valeurs sera typiquement inclus dans $q^{\mathbf{Z}}$ où $q$ est le cardinal du corps des constantes. On a donc dans ce cas

$$
\forall n \in \mathbf{N}, \quad n_{V, H}\left(q^{n+\frac{1}{2}}\right)=n_{V, H}\left(q^{n}\right)
$$

et une formule du type (1.2) entraînerait alors aussitôt la contradiction $\sqrt{q}=$ 1 .

Pour obtenir une version fonctionnelle satisfaisante de la question 1.1, on remarque que le comportement asymptotique de $n_{U, H}(B)$ est étroitement lié, par des théorèmes taubériens, au comportement analytique de la série génératrice

$$
\zeta_{U, H}(s)=\sum_{x \in U(K)} H(x)^{-s}
$$

( $s$ désignant une variable complexe), que l'on baptise fonction zêta des hauteurs. Un des moyens couramment utilisés pour obtenir une formule du type (1.2) est d'ailleurs d'étudier d'abord le comportement analytique de cette fonction, puis d'appliquer un théorème taubérien adéquat, tel que le résultat suivant.

\section{Théorème 1.2}

$S$ 'il existe un ouvert $U$ non vide tel que $\zeta_{U, H}(s)$ converge absolument pour $\Re(s)>1$ et un nombre réel $\varepsilon>0$ tels que la fonction

$$
s \longmapsto(s-1)^{t} \zeta_{U, H}(s)
$$

se prolonge en une fonction $g$ holomorphe sur l'ouvert $\{\Re(s)>1-\varepsilon\}$, et vérifiant $g(1) \neq 0$ alors la formule (1.2) est vérifiée pour cet ouvert $U$ avec $C=\frac{g(1)}{(t-1) !}$. 
La question qui suit peut alors être vue comme une version fonctionnelle de la question 1.1.

\section{Question 1.3}

Soit $V$ une variété projective et lisse définie sur un corps global $K$ de caractéristique positive. On suppose que la classe du faisceau anticanonique est à l'intérieur du cône effectif, et que l'ensemble $V(K)$ des points rationnels de $V$ est dense pour la topologie de Zariski. Soit $t$ le rang du groupe de Néron-Séveri de $V$. Soit $H$ une hauteur relative au faisceau anticanonique.

Existe-t-il un ouvert de Zariski non vide $U$ de $V$ tel que la série

$$
\zeta_{U, H}(s)=\sum_{x \in U(K)} H(x)^{-s}
$$

converge absolument pour $\Re(s)>1$ et, pour un certain $\varepsilon>0$, se prolonge en une fonction méromorphe sur l'ouvert $\{\Re(s)>1-\varepsilon\}$, qui a un pôle d'ordre $t$ en $s=1$ ?

Naturellement, et en accord avec les remarques déjà faites, même si cette question admet une réponse positive, on ne pourra pas appliquer le théorème 1.2. En caractéristique non nulle, la fonction zêta des hauteurs a d'autres pôles que 1 sur la droite $\Re(s)=1$, ne serait-ce que ceux provenant de la périodicité de $H$.

Dans le cas des corps de nombres, Peyre a été le premier dans [Pe1] à proposer (moyennant quelques hypothèses supplémentaires sur la variété $V$ ) une expression conjecurale de la constante $C$ apparaissant dans la formule (1.2). Cette expression conjecturale dépend d'invariants géométriques et arithmétiques de la variété $V$, ainsi que du choix de la hauteur. Elle a ensuite été raffinée par Batyrev et Tschinkel, et adaptée au cas fonctionnel par Peyre dans [Pe3]. Nous rappelons la définition de la constante de Peyre raffinée à la section 2.3. Nous la noterons $C_{V, H}^{*}$.

On a ainsi des versions raffinées des questions 1.1 et 1.3 .

\section{Question 1.4}

Soit $V$ une variété projective et lisse définie sur un corps de nombres $K$. On suppose que la classe du faisceau anticanonique est à l'intérieur du cône effectif, et que l'ensemble $V(K)$ des points rationnels de $V$ est dense pour la topologie de Zariski. Soit $t$ le rang du groupe de Néron-Séveri de $V$. Soit $H$ une hauteur relative au faisceau anticanonique. On suppose en outre que $V$ vérifie les hypothèses nécessaires pour que la constante de Peyre raffinée $C_{V, H}^{*}$ soit définie.

Existe-t-il un ouvert de Zariski non vide $U$ de $V$ tel qu'on ait

$$
n_{U, H}(B) \underset{B \rightarrow+\infty}{\sim} C_{V, H}^{*} B \log (B)^{t-1} \quad ?
$$


Remarque 1.5 : À la connaissance de l'auteur, dans tous les cas où on sait montrer que la réponse à la question 1.1 est positive, on sait également montrer que la réponse à la question 1.4 est positive.

\section{Question 1.6}

Soit $V$ une variété projective et lisse définie sur un corps global $K$ de caractéristique positive. On suppose que la classe du faisceau anticanonique est à l'intérieur du cône effectif, et que l'ensemble $V(K)$ des points rationnels de $V$ est dense pour la topologie de Zariski. Soit $t$ le rang du groupe de Néron-Séveri de $V$. Soit $H$ une hauteur relative au faisceau anticanonique, On suppose en outre que $V$ vérifie les hypothèses nécessaires pour que la constante de Peyre raffinée $C_{V, H}^{*}$ soit définie.

Existe-t-il un ouvert de Zariski non vide $U$ de $V$ tel que la série

$$
\zeta_{U, H}(s)=\sum_{x \in U(K)} H(x)^{-s}
$$

converge absolument pour $\Re(s)>1$ et, pour un certain $\varepsilon>0$, se prolonge en une fonction méromorphe sur l'ouvert $\{\Re(s)>1-\varepsilon\}$, qui a un pôle d'ordre $t$ en $s=1$, et vérifiant

$$
\lim _{s \rightarrow 1}(s-1)^{t} \zeta_{U, H}(s)=(t-1) ! C_{V, H}^{*} \quad ?
$$

Concernant la question 1.6, le cas des espaces projectifs est traité par Wan dans [Wa], montrant ainsi une formule figurant déjà dans [Se2]. Le cas des variétés de drapeaux, qui englobe le précédent, a été traité indépendamment par Peyre dans [Pe3], et Lai et Yeung dans [LaYe] (sans interprétation de la constante dans ce dernier cas, c'est-à-dire que seule la question 1.3 est considérée).

Dans ce texte, on étudie la question 1.6 pour une variété torique projective et lisse définie sur un corps global de caractéristique positive, non nécessairement déployée.

Une de motivations de ce travail est que le problème analogue sur les corps de nombres a déjà été traité avec succès ${ }^{1}$, qui plus est de deux manière différentes : Batyrev et Tschinkel ont démontré dans [BaTs1] et [BaTs2] que la réponse à la question 1.4 était positive pour les variétés toriques, en exploitant la structure de groupe du tore pour utiliser des techniques d'analyse harmonique. Par la suite Salberger a redémontré dans [Sa] le résultat dans un cadre plus restreint (variétés toriques déployées, définies sur $\mathbf{Q}$, de faisceau anticanonique globalement engendré) mais par une méthode complètement

\footnotetext{
${ }^{1}$ C'était également le cas pour les variétés de drapeaux.
} 
différente basée sur l'usage de la description explicite des torseurs universels au-dessus des variétés toriques.

Dans [Bo1] et [Bo2], nous avons montré comment, en s'inspirant de la méthode de Salberger, on pouvait montrer que la réponse à la question 1.6 était positive pour les variétés toriques déployées définies sur un corps de fonctions quelconque (sans hypothèse sur le faisceau anticanonique).

Dans ce texte, nous adaptons au cas fonctionnel l'approche utilisée par Batyrev et Tschinkel dans [BaTs1] et [BaTs2], pour étendre le résultat aux variétés toriques non nécessairement déployées. La sous-section suivante détaille cette adaptation.

Ce texte contient également une présentation détaillée de la démonstration du résultat de Batyrev et Tschinkel, les deux démonstrations étant présentées en parallèle. La raison de ce choix est au moins double : tout d'abord, il permet de bien mettre en évidence les analogies et les différences qui existent dans le traitement du calcul de la fonction zêta des hauteurs des variétés toriques entre le cas des corps de nombres et le cas des corps de fonctions. Ensuite, pour autant qu'il nous soit permis d'en juger, ce choix peut s'avérer utile à ceux qui désirent comprendre en détail la démarche de Batyrev et Tschinkel, les articles [BaTs1] et [BaTs2] pouvant s'avérer d'un abord un peu ardu et elliptique pour le lecteur non averti.

\section{Remerciements}

Je remercie chaleureusement Antoine Chambert-Loir, Jean-Louis ColliotThélène et Emmanuel Peyre pour leurs remarques, corrections et suggestions concernant ce texte. J'ai envers le rapporteur une reconnaissance tout particulière pour sa lecture minutieuse et ses innombrables corrections et suggestions.

\subsection{L'adaptation de la méthode de Batyrev et Tschinkel en caractéristique positive}

Dans cette section, nous résumons brièvement la méthode utilisée dans [BaTs2] et [BaTs1], en expliquant quelles parties de la démonstration nécessitent une modification en caractéristique non nulle.

La première étape consiste à définir explicitement un système de hauteurs puis à l'étendre à l'espace adélique associé au tore. La construction est strictement la même dans le cas fonctionnel. Elle est rappelée dans les sections 4.2 (nous corrigeons au passage une erreur de Batyrev et Tschinkel dans la définition des hauteurs locales pour les places ramifiées) et 4.5. 
À ce stade, il faut déjà noter que la topologie de l'espace adélique associé au tore a des propriétés différentes dans chacune des deux situation. Moralement, en fait, la situation est plus agréable en caractéristique positive : beaucoup des groupes topologiques mis en jeu sont compacts (notamment, le point 3 de la proposition 3.24 n'est valable qu'en caractéristique positive).

Disposant des fonctions hauteurs sur l'espace adélique associé au tore, lequel est un groupe abélien localement compact, l'idée cruciale de Batyrev et Tschinkel est d'appliquer la formule de Poisson afin d'obtenir une représentation intégrale de la fonction zêta des hauteurs. Pour ce faire, il faut établir l'intégrabilité de la transformée de Fourier de la hauteur, laquelle se décompose en produit de transformées de Fourier locales. On utilise dans le cas des corps de nombres une expression explicite pour presque toutes les transformées de Fourier locales (cf. le théorème 5.27), et des majorations adéquates pour les transformées de Fourier restantes. La formule explicite décrivant presque toutes les transformées de Fourier locales est la même dans le cas fonctionnel. En ce qui concerne les transformées de Fourier locales aux places restantes, leur continuité suffit pour assurer la convergence dans le cas fonctionnel, cependant nous avons besoin de quelques renseignements sur la forme des fonctions obtenues (cf. la sous-section 5.4.3).

Le choix d'un scindage du groupe des caractères du tore permet alors de montrer que la fonction zêta des hauteurs s'obtient par intégration (sur un espace vectoriel réel dans le cas arithmétique, sur un produit de cercles dans le cas fonctionnel) d'une fonction qui possède une expression en terme de produit de fonctions $L$ de Hecke; cf. le corollaire 5.48 pour le cas des corps de nombres (cf. également [BaTs1, Theorem 3.1.3] et [BaTs2, page 46]), et le corollaire 5.54 pour le cas fonctionnel. Il faut «maîtriser» le comportement analytique de le fonction sous l'intégrale, et dans le cas des corps de nombres, on a besoin pour cela d'un contrôle uniforme sur les bandes verticales des fonctions $L$, obtenu par Rademacher via le principe de Phragmen-Lindelöf (proposition 5.43 et [BaTs1, Theorem 3.2.3]). Dans le cas fonctionnel, l'holomorphie de la fonction $L(., \chi)$ quand le caractère $\chi$ est non trivial est suffisante.

Pour déterminer les propriétés analytiques la fonction zêta des hauteurs, il s'agit maintenant de comprendre comment l'intégration modifie le comportement analytique de la fonction sous l'intégrale (cet étape n'apparaît d'ailleurs pas dans le cas des tores anisotropes). C'est l'objet de la proposition technique de Batyrev et Tschinkel ([BaTs2, Theorem 6.19]). La démonstration procède par des applications successives du théorème des résidus. Dans ce texte, nous utilisons une version raffiné du résultat dûe à Chambert-Loir et Tschinkel (théorème 6.2).

La transposition directe du lemme technique et de sa démonstration en 
caractéristique non nulle s'avère difficile à mettre en œuvre, car bien que la compacité des espaces topologiques mis en jeu simplifie un peu les choses, rendant inutiles des hypothèses du type contrôle uniforme sur les bandes verticales (indispensables en caractéristique zéro), les fonctions zêtas des hauteurs en caractéristique non nulle s'avèrent posséder plus de pôles sur la droite $\Re(s)=1$ que ceux provenant de la périodicité de la hauteur. Ce phénomène est même déjà visible dans le cas des variétés toriques déployées. Prenons en effet l'exemple du plan projectif éclaté en un point : en notant $q$ le cardinal du corps des constantes, la formule de la page 355 de [Bo1] montre que la fonction zêta des hauteurs anticanonique s'écrit dans ce cas

$$
\zeta_{H}(s)=f_{1}\left(q^{-s}\right) \zeta_{\mathcal{C}}(3 s-2) \zeta_{\mathcal{C}}(2 s-1)+f_{2}\left(q^{-s}\right) \zeta_{\mathcal{C}}(2 s-1)+f_{3}\left(q^{-s}\right),
$$

où, pour $i=1,2,3, s \mapsto f_{i}\left(q^{-s}\right)$ est holomorphe sur le domaine $\Re(s)>\frac{1}{2}$ et $s \mapsto f_{1}\left(q^{-s}\right)$ ne s'annule pas sur ce domaine. Ainsi l'ensemble des pôles situés sur la droite $\Re(s)=1$ contient $\left\{1+\frac{2 i k \pi}{3 \log q}\right\}_{k \in \mathbf{Z}}$, bien qu'on puisse vérifier que la fonction zêta des hauteurs n'est pas $\frac{2 i \pi}{3 \log q}$-périodique. Rappelons que dans le cas de la caractéristique zéro, Batyrev et Tschinkel montrent que le seul pôle de la fonction zêta des hauteurs des variétés toriques sur la droite $\Re(s)=1$ est $s=1$. La gestion des pôles supplémentaires en caractéristique non nulle se révèle vite être très délicate (voire ingérable...) si on veut suivre «au plus près» la méthode de Batyrev et Tschinkel.

C'est pourquoi, pour aboutir à une version fonctionnelle du résultat technique utilisé dans le cas des corps de nombres, nous exploitons la périodicité des fonctions mises en jeu pour les exprimer en terme de séries de type combinatoire. Les techniques utilisées pour évaluer le comportement de ces séries par intégration sont alors similaires à celles employées dans [Bo2]. Nous aboutissons ainsi aux lemmes 7.10, 7.12 et 7.14 qui sont le pendant en caractéristique positive du lemme technique de Batyrev et Tschinkel.

Le comportement analytique de la fonction obtenue après intégration est essentiellement décrit par une suite exacte de $\mathbf{Z}$-modules libres de rang fini (cf. les énoncés du théorème 6.2 et du lemme 7.8). La suite exacte mise en jeu n'est pas exactement la même dans le cas des corps de nombres ou dans le cas fonctionnel. Dans les deux cas, elle provient de la construction suivante : la résolution flasque du groupe des caractères du tore par le groupe de Picard de la variété torique (i.e. la suite exacte (4.11)). induit par dualité une suite exacte de tores algébriques; on considère alors l'image de cette suite exacte par le morphisme degré (définie à la section 3.3). La différence essentielle vient alors du fait que le morphisme degré est surjectif dans le cas des corps de nombres mais pas dans le cas fonctionnel (cf. le lemme 3.15) où il est seulement de conoyau fini. 
La dernière étape de la démonstration consiste à calculer explicitement le terme principal de la fonction zêta des hauteurs au point critique $s=1$, et à vérifier s'il coïncide avec la prédiction de Peyre. On a besoin d'un théorème d'Ono sur le nombre de Tamagawa d'un tore algébrique (théorème 3.41), lequel théorème a été démontré dans [On3] pour tout corps global, mais a cependant dû être corrigé par Oesterlé dans le cas de la caractéristique non nulle, en introduisant un facteur correctif dans la définition du nombre de Tamagawa (ce facteur correctif provient de la non-surjectivité du degré en caractéristique non nulle). On a besoin également de résultats de ColliotThélène et Sansuc, démontrés pour tout corps global également, et permettant d'obtenir le lemme 3.32. On peut donc ici reprendre la ligne de calcul de Batyrev et Tschinkel, ce qui est fait dans la partie 8. Notons que dans le cas fonctionnel, le défaut de surjectivité du degré fait intervenir au cours du calcul des termes non triviaux (correspondant à des cardinaux de groupes finis) qui n'apparaissent pas dans le cas des corps de nombres. Dans une version précédente de ce texte, l'auteur affirmait que l'un de ces termes non triviaux subsistait dans l'expression finale du terme principal de la fonction zêta des hauteurs (il s'agit de l'invariant $\mathcal{K}_{T}$ défini à la sous-section 3.8.4), «montrant» ainsi que la constante prédite par Peyre et Batyrev-Tschinkel n'était pas la bonne dans ce cas. Les calculs avaient été menés en supposant à tort que le groupe intervenant dans le point 3 du lemme 5.41 était trivial. Une fois ce point corrigé, on s'aperçoit que tous les termes supplémentaires intervenant en caractéristique non nulle dans le calcul du terme principal de la fonction zêta des hauteurs se simplifient, et que l'expression obtenue est bien celle attendue.

\section{Rappels et notations}

\subsection{Quelques notations}

Nous fixons ici quelques notations utilisées dans l'ensemble du texte.

On note $[E]$ le cardinal d'un ensemble fini $E$.

Pour tout réel $\alpha$, on note $\mathbf{R}_{>\alpha}$ l'ensemble $\{x \in \mathbf{R}, x>\alpha\}$. On définit de même de manière évidente les ensembles $\mathbf{R}_{\geqslant \alpha}, \mathbf{R}_{<\alpha}$ et $\mathbf{R}_{\leqslant \alpha}$.

On note, pour tout corps $K, \bar{K}$ une clôture algébrique de $K$ et $K^{\text {s }}$ la clôture séparable de $K$ dans $\bar{K}$.

Soit $N$ un groupe abélien. On note $N^{\vee}$ le dual algébrique de $N$, c'està-dire le groupe abélien $\operatorname{Hom}(N, \mathbf{Z})$. L'accouplement naturel entre $N$ et $N^{\vee}$ sera noté $\langle.,$.$\rangle .$

Soit $M$ un autre groupe abélien et $f: M \rightarrow N$ un morphisme de groupes 
Pour tout anneau commutatif unitaire $A$, on note $N_{A}$ le $A$-module $N \otimes_{\mathbf{z}} A$ et $f_{A}$ le morphisme de $A$-module $f \otimes$ Id $: M_{A} \rightarrow N_{A}$ induit par $f$.

Pour tout anneau commutatif unitaire $A$, on désigne par $A^{\times}$le groupe des éléments inversibles de $A$.

Si $N$ est un Z-module libre de rang fini, et $U$ est une partie de $N_{\mathbf{R}}$, on note

$$
\mathcal{T}(U) \stackrel{\text { déf }}{=}\left\{s \in N_{\mathbf{C}}, \Re(s) \in U\right\}
$$

le domaine tubulaire au-dessus de $U$.

\subsection{Rappels sur les corps globaux}

Dans tout ce texte, on appellera corps de fonctions un corps global de caractéristique positive, i.e. une extension $K$ de type fini et de degré de transcendance 1 d'un corps fini. Le corps des constantes de $K$ est la clôture algébrique du sous-corps premier de $K$ dans $K$. Il sera noté $\mathbf{F}_{K}$. Le choix d'une base de transcendance séparable de $K$ sur $\mathbf{F}_{K}$ permet alors d'identifier $K$ à une extension finie séparable du corps des fractions rationnelles en une indéterminée $\mathbf{F}_{K}(T)$. Il existe en outre une courbe projective, lisse et géométriquement intègre définie sur $\mathbf{F}_{K}$, unique à isomorphisme près, et notée $\mathcal{C}_{K}$, telle que $K$ s'identifie au corps de fonctions de $\mathcal{C}_{K}$.

On adopte dans ce texte la convention suivante : on fixe un corps fini $k$ dont on note $q$ le cardinal. On supposera alors, dans tout ce texte, que les corps de fonctions considérés ont un corps des constantes qui contient $k$. Pour un tel corps de fonctions $K$ on note $q_{K}$ le cardinal du corps des constantes, et $d_{K}$ son degré absolu, c'est-à-dire que $d_{K}$ vérifie $q_{K}=q^{d_{K}}$.

Soit $K$ un corps global, i.e. un corps de nombres ou un corps de fonctions. Dans tout ce texte, on dira être dans le cas arithmétique si le corps de base est un corps de nombres et dans le cas fonctionnel si c'est un corps de fonctions. Dans le cas arithmétique, on note $\operatorname{disc}(K)$ le discriminant absolu de $K$ et dans le cas fonctionnel on note $g_{K}$ le genre de $K$, c'est-à-dire le genre de $\mathcal{C}_{K}$.

On note $\mathcal{P}_{K}$ l'ensemble des places de $K$, et $\mathcal{P}_{K, f}$ (respectivement $\mathcal{P}_{K, \infty}$ ) l'ensemble des places finies (respectivement archimédiennes) de $K$. On identifiera toujours un élément $v$ de $\mathcal{P}_{K, f}$ à l'unique valuation normalisée qui le représente, i.e. l'unique élément de $v$ dont le groupe de valeurs est $\mathbf{Z}$. Dans le cas fonctionnel, on a $\mathcal{P}_{K}=\mathcal{P}_{K, f}$ et $\mathcal{P}_{K}$ s'identifie à l'ensemble des points fermés de la courbe $\mathcal{C}_{K}$.

Pour $v \in \mathcal{P}_{K}$, on note $K_{v}$ le complété de $K$ en $v$. Pour $v \in \mathcal{P}_{K, f}$, on note également $\mathcal{O}_{v}$ l'anneau de valuation de $v$, et $k_{v}$ le corps résiduel de $v$.

Pour $v \in \mathcal{P}_{K, \infty}$, on désignera abusivement par $\mathcal{O}_{v}^{\times}$le sous-groupe de $K_{v}^{\times}$ constitué des éléments de valeur absolue 1. 
Dans le cas fonctionnel, on note aussi

$$
f_{v}=\left[k_{v}: \mathbf{F}_{K}\right]
$$

le degré résiduel «absolu».

Le cardinal de $k_{v}$ sera noté $q_{v}$. Dans le cas fonctionnel, il est égal à $q_{K}^{f_{v}}$.

On normalise la mesure de Haar $d x_{v}$ sur $K_{v}$ de la manière suivante : si $v$ est finie, on choisit $d x_{v}$ de sorte qu'on ait la relation $\int_{\mathcal{O}_{v}} d x_{v}=1$, si $v$ est archimédienne et $K_{v}=\mathbf{R}, d x_{v}$ est la mesure de Lebesgue usuelle et si $K_{v}$ est isomorphe à $\mathbf{C}$ on prend $d x_{v}=i d z d \bar{z}$.

Si $S$ est un sous-ensemble fini de $\mathcal{P}_{K}$ contenant $\mathcal{P}_{K, \infty}$, on note

$$
\mathcal{O}_{S}=\bigcap_{v \notin S} \mathcal{O}_{v}
$$

l'anneau des $S$-entiers de $K$.

Soit $v \in \mathcal{P}_{K, f}$. Pour $x \in K_{v}$ on note

$$
|x|_{v}=q_{v}^{-v(x)} .
$$

Si $v \in \mathcal{P}_{K} \backslash \mathcal{P}_{K, f},|.|_{v}$ désigne la valeur absolue usuelle sur $\mathbf{R}$ si $K_{v}=\mathbf{R}$, et le carré de la valeur absolue usuelle sur $\mathbf{C}$ si $K_{v}$ est isomorphe à $\mathbf{C}$.

Avec ce choix de valeur absolues, on a, pour tout $v \in \mathcal{P}_{K}$ et pour tout $a$ de $K_{v}, d(a x)_{v}=|a|_{v} d x_{v}$. Pour tout $x \in K^{\times}$on a $|x|_{v}=1$ pour tout $v \in \mathcal{P}_{K}$ sauf un nombre fini et la formule du produit

$$
\prod_{v \in \mathcal{P}_{K}}|x|_{v}=1
$$

La fonction zêta de Dedekind de $K$, notée $\zeta_{K}$, est définie par la formule

$$
\zeta_{K}(s)=\prod_{v \in \mathcal{P}_{K, f}}\left(1-q_{v}^{-s}\right)^{-1}
$$

où $s$ est une variable complexe. On dispose des résultats classiques suivants sur le comportement analytique de $\zeta_{K}$.

\section{Proposition 2.1}

1. Dans le cas arithmétique, la série définissant $\zeta_{K}(s)$ converge absolument pour $\Re(s)>1$ et se prolonge en une fonction méromorphe sur $\boldsymbol{C}$ tout entier, avec un pôle simple en $s=1$. La fonction $(s-1) \zeta_{K}(s)$ se prolonge en une fonction holomorphe sur $\mathcal{T}\left(\boldsymbol{R}_{>0}\right)$. 
2. Dans le cas fonctionnel, la série définissant $\zeta_{K}(s)$ converge absolument pour $\Re(s)>1$ et se prolonge en une fonction méromorphe sur $\boldsymbol{C}$ tout entier, avec un pôle simple en $s=1$, et sans zéro pour $\Re(s) \neq \frac{1}{2}$. De plus $\zeta_{K}(s)$ est une fraction rationnelle en $q_{K}^{-s}$, plus précisément on a

$$
\zeta_{K}(s)=\frac{P\left(q_{K}^{-s}\right)}{\left(1-q_{K}^{-s}\right)\left(1-q_{K}^{1-s}\right)}
$$

où $P$ est un polynôme.

Dans le cas fonctionnel, on note $Z_{K}$ la fraction rationnelle vérifiant

$$
\forall s \in \mathcal{T}\left(R_{>1}\right), \quad Z_{K}\left(q_{K}^{-s}\right)=\zeta_{K}(s) .
$$

Nous faisons à présent quelques rappels sur une généralisation naturelle de la fonction zêta de Dedekind : les fonctions $L$ d'Artin.

Soit $K$ un corps global et $M$ un $\mathbf{Z}$-module de rang fini muni d'une structure de $\operatorname{Gal}\left(K^{\mathrm{s}} / K\right)$-module discret, c'est-à-dire une représentation

$$
\rho: \operatorname{Gal}\left(K^{\mathrm{s}} / K\right) \longrightarrow \operatorname{Aut}(M)
$$

se factorisant à travers un quotient fini de $\operatorname{Gal}\left(K^{\mathrm{s}} / K\right)$.

Soit $G$ un tel quotient, qui est donc le groupe de Galois d'une extension finie galoisienne $L$ de $K$. On a une représentation

$$
\rho: G \longrightarrow \operatorname{Aut}(M) \text {. }
$$

Soit $v$ une place finie de $K, G_{v}$ un groupe de décomposition au-dessus de $v$ et $I_{v}$ le groupe d'inertie correspondant. Soit

$$
\rho_{v}: G_{v} / I_{v} \rightarrow \operatorname{Aut}\left(M^{I_{v}}\right)
$$

la représentation déduite de $\rho$. Soit $\operatorname{Fr}_{v} \in G_{v} / I_{v}$ le frobenius. On pose, pour tout nombre complexe $s$ tel que $\Re(s)>0$,

$$
L_{v}(s, M, \rho)=\frac{1}{\operatorname{det}\left(1-\rho_{v}\left(\operatorname{Fr}_{v}\right) q_{v}^{-s}\right)},
$$

ce qui est bien défini et ne dépend ni du choix de $G_{v}$, ni du choix de $G$. Si $v$ est une place archimédienne, par commodité d'écriture, on notera $L_{v}(s, M)$ la fonction constante égale à 1 .

Exemple 2.2 : Si $M$ est de rang 1 et $\rho$ est la représentation triviale, alors pour toute place $v$ finie on a $L_{v}(s, M, \rho)=\left(1-q_{v}^{-s}\right)^{-1}$, en d'autres termes $L_{v}(s, M, \rho)$ coïncide avec le facteur local en $v$ du produit eulérien définissant la fonction $\zeta_{K}$. 


\section{Proposition 2.3}

1. Le produit eulérien

$$
s \mapsto \prod_{v \in \mathcal{P}_{K}} L_{v}(s, M, \rho)
$$

converge normalement sur tout compact de $\mathcal{T}\left(\boldsymbol{R}_{>1}\right)$, et définit donc une fonction holomorphe sur $\mathcal{T}\left(\boldsymbol{R}_{>} 1\right)$, notée $L(s, M, \rho)$.

2. La fonction $L(., M, \rho)$ se prolonge en une fonction méromorphe sur $\boldsymbol{C}$, ayant un pôle d'ordre $\operatorname{rg}\left(M^{\rho\left(\operatorname{Gal}\left(K^{s} / K\right)\right)}\right)$ en $s=1$.

Démonstration: Le résultat est vrai si $\rho$ est la représentation triviale, d'après l'exemple 2.2 et la proposition 2.1. Si $\rho$ est irréductible et $M$ est de rang supérieur à 2 , d'après [Ar, Satz 3] le résultat est encore vrai et $L(., M, \rho)$ se prolonge en fait en une fonction méromorphe sur tout le plan complexe qui est holomorphe et inversible au voisinage de 1 .

Dans le cas général, soit $G$ un quotient fini de $\operatorname{Gal}\left(K^{\mathrm{s}} / K\right)$ à travers lequel $\rho$ se factorise. On a une décomposition

$$
M_{\mathbf{C}}=M_{\mathbf{C}}^{\rho(G)} \bigoplus \underset{i \in I}{\oplus} M_{i}
$$

où, pour $i \in I, M_{i}$ est un sous-espace $\rho(G)$-stable de dimension supérieur à 2 et la représentation $\rho_{i}: G \rightarrow \operatorname{Aut}\left(M_{i}\right)$ est irréductible. On a alors pour toute place finie $v$

$$
L_{v}(s, M, \rho)=\left(\frac{1}{1-q_{v}^{-s}}\right)^{\mathrm{rg}\left(M^{\rho(G)}\right)} \prod_{i \in I} L_{v}\left(s, M_{i}, \rho_{i}\right) .
$$

Le résultat en découle.

On pose

$$
\ell(M, \rho)=\lim _{s \rightarrow 1}(s-1)^{\operatorname{rg}\left(M^{\rho\left(\operatorname{Gal}\left(K^{\mathrm{s}} / K\right)\right)}\right)} L(s, M, \rho)
$$

Par la suite on notera très souvent $L_{v}(s, M)$ (respectivement $L(s, M)$, respectivement $\ell(M)$ ) en lieu et place de $L_{v}(s, M, \rho)$ (respectivement $L(s, M, \rho)$, respectivement $\ell(M, \rho))$ lorsque la représentation $\rho$ sera clairement indiquée par le contexte.

On énonce à présent deux lemmes élémentaires sur ces fonctions $L$.

\section{Lemme 2.4}

Soit

$$
0 \longrightarrow M_{1} \longrightarrow M_{2} \longrightarrow M_{3} \longrightarrow 0
$$


une suite exacte de $\operatorname{Gal}\left(K^{\mathrm{s}} / K\right)$-modules discrets qui sont libres de rang fini en tant que $\mathbf{Z}$-modules. On a alors pour toute place $v$ de $K$

$$
\forall s \in \mathcal{T}\left(\boldsymbol{R}_{>0}\right), \quad L_{v}\left(s, M_{2}\right)=L_{v}\left(s, M_{1}\right) L_{v}\left(s, M_{3}\right)
$$

En particulier, on a

$$
\forall s \in \mathcal{T}\left(\boldsymbol{R}_{>1}\right), \quad L\left(s, M_{2}\right)=L\left(s, M_{1}\right) L\left(s, M_{3}\right)
$$

et

$$
\ell\left(M_{2}\right)=\ell\left(M_{1}\right) \ell\left(M_{3}\right)
$$

\section{Lemme 2.5}

Soit $L / K$ une extension finie galoisienne de groupe $G, H$ un sous-groupe de $G, K^{\prime}$ le corps global $K^{H}$. Alors, pour toute place $v$ de $K$ et tout $s \in \mathcal{T}\left(\boldsymbol{R}_{>0}\right)$, on a

$$
L_{v}(s, \boldsymbol{Z}[G / H])=\prod_{\substack{w \in \mathcal{P}_{K^{\prime}} \\ w \mid v}} \frac{1}{1-q_{w}^{-s}}
$$

En particulier, on a

$$
L(., Z[G / H])=\zeta_{K^{\prime}}
$$

et

$$
\ell(\boldsymbol{Z}[G, H])=\operatorname{Res}_{s=1} \zeta_{K^{\prime}}(s)
$$

\subsection{Hauteurs d'Arakelov, mesure de Tamagawa et con- stante de Peyre}

Dans cette section, nous rappelons brièvement la construction des hauteurs d'Arakelov sur une variété projective définie sur un corps global à partir de métriques adéliques sur les fibrés en droites, renvoyant à [Pe4, chapitre 2] pour plus de détails, notamment les preuves omises. Suivant Peyre ([Pe1] et [Pe3]), nous expliquons ensuite comment une métrique adélique sur le faisceau anticanonique d'une variété projective lisse $V$ définie sur un corps global $K$ induit une mesure sur l'espace adélique $V\left(\mathbf{A}_{K}\right)$ associé à $V$. La définition générale de l'espace adélique associé à une variété algébrique est traitée dans [We2, I.2] et [Oe, I.3]. Ici, comme $V$ est projective, $V\left(\mathbf{A}_{K}\right)$ est l'espace

$$
\prod_{v \in \mathcal{P}_{K}} V\left(K_{v}\right)
$$

muni de la topologie produit. 
Enfin nous rappelons comment la mesure ainsi construite sur $V\left(\mathbf{A}_{K}\right)$ permet alors, si $V$ vérifie des hypothèses supplémentaires, de définir la constante $C_{V, H}^{*}$ apparaissant dans les questions 1.4 et 1.6. Là encore, nous renvoyons à [Pe1] et [Pe3] pour plus de détails.

Soit $V$ une variété projective, lisse et géométriquement intègre définie sur un corps global $K$, et soit $\mathcal{L}$ un fibré en droites sur $V$. Si $v$ est une place de $K$, une métrique $v$-adique sur $\mathcal{L}$ est la donnée, pour tout point $K_{v}$-rationnel $x: \operatorname{Spec}\left(K_{v}\right) \rightarrow V$, d'une norme $v$-adique $\|.\|_{v, x}$ sur $\mathcal{L}(x)=x^{*} \mathcal{L}$ (rappelons que l'on a en particulier $\|\left. a s\right|_{v, x}=\left.|a|_{v}|| s\right|_{v, x}$ pour tout $s$ de $\mathcal{L}(x)$ et tout $a$ de $K_{v}$ ), telle que pour tout ouvert de Zariski $U$ de $V$ et toute section $s$ de $\mathcal{L}$ sur $U$, l'application

$$
x \mapsto\|s(x)\|_{v, x}
$$

est continue sur $U\left(K_{v}\right)$ pour la topologie $v$-adique.

Remarque 2.6 : Si $v$ est une place finie de $K$, la donnée d'un modèle projectif $\mathcal{V}$ de $V \operatorname{sur} \operatorname{Spec}\left(\mathcal{O}_{v}\right)$ et d'un modèle $\mathcal{L}$ de $\mathcal{L}$ sur $\mathcal{V}$ définit de manière naturelle une telle métrique. Soit en effet $x \in V\left(K_{v}\right)$. Par le critère valuatif de propreté, $x$ définit un point $\widetilde{x}: \operatorname{Spec}\left(\mathcal{O}_{v}\right) \rightarrow \mathcal{V}$. Le $\mathcal{O}_{v}$-module $\mathcal{L}_{\widetilde{x}}=\widetilde{x}^{*} \mathcal{L}$ est alors libre de rang 1. Soit $s_{0}$ un générateur. Le changement de base $\mathcal{O}_{v} \rightarrow K_{v}$ induit un isomorphisme naturel de $\mathcal{L}(x)$ sur $\mathcal{L}_{\widetilde{x}} \otimes_{\mathcal{O}_{v}} K_{v}$, qui permet de définir une norme $v$-adique sur $\mathcal{L}(x)$ par la formule

$$
\forall s \in \mathcal{L}(x), \quad\|s\|_{v}=\left|\frac{s}{s_{0}}\right|_{v} .
$$

Comme deux générateurs de $\mathcal{L}_{\widetilde{x}}$ se déduisent l'un de l'autre par multiplication par un élément inversible de $\mathcal{O}_{v}$, cette définition est bien indépendante du choix de $s_{0}$.

Une métrique adélique sur $\mathcal{L}$ est alors la donnée d'une famille $\left(\|.\|_{v}\right)_{v \in \mathcal{P}_{K}}$ de métriques $v$-adiques sur $\mathcal{L}$ vérifiant la condition suivante : il existe un ensemble fini $S$ de places de $K$ contenant les places archimédiennes, un modèle projectif $\mathcal{V}$ de $V$ sur $\operatorname{Spec}\left(\mathcal{O}_{S}\right)$, et un modèle $\mathcal{L}$ de $\mathcal{L}$ sur $\mathcal{V}$, tels que pour presque tout $v$ de $\mathcal{P}_{K} \backslash S$ la métrique $\|.\|_{v}$ est définie par le couple $\left(\mathcal{V} \times_{\operatorname{Spec}\left(\mathcal{O}_{S}\right)} \operatorname{Spec}\left(\mathcal{O}_{v}\right), \mathcal{L} \times_{\operatorname{Spec}\left(\mathcal{O}_{S}\right)} \operatorname{Spec}\left(\mathcal{O}_{v}\right)\right)$.

Donnons un exemple d'une métrique adélique sur $\mathcal{L}$ lorsque $\mathcal{L}$ est engendré par ses sections globales. On fixe une base $\left\{s_{0}, \ldots, s_{r}\right\}$ du $K$-espace vectoriel $H^{0}(X, \mathcal{L})$. On pose alors, pour tout $v \in \mathcal{P}_{K}$, pour tout $x \in V\left(K_{v}\right)$ et toute section locale $s$ de $\mathcal{L}$ ne s'annulant pas en $x$

$$
\|s(x)\|_{v, x}^{-1}=\operatorname{Max}_{i=0, \ldots, r}\left(\left|\frac{s_{i}(x)}{s(x)}\right|_{v}\right) .
$$

Il est à noter que cette définition ne dépend pas du choix de la base de $H^{0}(X, \mathcal{L})$. 


\section{Lemme 2.7}

La famille de métriques $v$-adiques ainsi définie est une métrique adélique sur $\mathcal{L}$.

\section{Définition 2.8}

Cette métrique adélique sera appelée métrique adélique standard sur $\mathcal{L}$.

Soit à présent $\mathcal{L}$ et $\mathcal{L}^{\prime}$ deux fibrés en droites sur $V$, et $\left(\|.\| \|_{v}\right)_{v \in \mathcal{P}_{K}}($ respectivement $\left.\left(\|.\|_{v}^{\prime}\right)_{v \in \mathcal{P}_{K}}\right)$ une métrique adélique sur $\mathcal{L}$ (respectivement $\mathcal{L}^{\prime}$ ).

Pour toute place $v$ de $K$, on définit ainsi la métrique $v$-adique produit $\|.\|_{v} \otimes\|.\|_{v}^{\prime}$ sur $\mathcal{L} \otimes \mathcal{L}^{\prime}:$ pour tout $x \in V\left(K_{v}\right)$, tout $s \in \mathcal{L}(x)$ et tout $s^{\prime} \in \mathcal{L}^{\prime}(x)$, on pose

$$
\left(\|\cdot\|_{v} \otimes\|\cdot\|_{v}^{\prime}\right)\left(s \otimes s^{\prime}\right)=\|s\|_{v, x}\left\|s^{\prime}\right\|_{v, x}^{\prime}
$$

On définit également la métrique $v$-adique duale $\|.\|_{v}^{\vee}$ sur $\mathcal{L}^{-1}$ : pour tout $x \in V\left(K_{v}\right)$ et tout $s^{\vee} \in \mathcal{L}^{-1}(x)$, on pose

$$
\left\|s^{\vee}\right\|_{v, x}^{\vee}=\frac{\left|\left\langle s^{\vee}, s\right\rangle\right|_{v}}{\|s\|_{v, x}}
$$

où $s$ est un élément quelconque de $\mathcal{L}(x) \backslash\{0\}$.

\section{Lemme 2.9}

$\left(\|.\|_{v} \otimes\|.\|_{v}^{\prime}\right)_{v \in \mathcal{P}_{K}}$ est une métrique adélique sur $\mathcal{L} \otimes \mathcal{L}^{\prime}$, et $\left(\|.\|_{v}^{\vee}\right)_{v \in \mathcal{P}_{K}}$ est une métrique adélique sur $\mathcal{L}^{-1}$.

Soit à présent $L$ une extension finie de $K$. On suppose donnée une métrique adélique $\left(\|.\|_{\mathcal{V}}\right)_{\mathcal{V} \in \mathcal{P}_{L}}$ sur $\mathcal{L}_{L}$. Pour tout $v \in \mathcal{P}_{K}$, soit $\mathcal{V}$ une place de $L$ divisant $v$. On identifie $V\left(K_{v}\right)$ à un sous-ensemble de $V\left(L_{\mathcal{V}}\right)$ Soit $x$ un élément de $V\left(K_{v}\right)$. On pose alors, pour tout élément $s$ de $\mathcal{L}(x)$,

$$
\|s\|_{v, x}=\|s\|_{\mathcal{V}, x}^{\frac{1}{\left.L_{\mathcal{V}}: K_{v}\right]}}
$$

\section{Lemme 2.10}

La métrique $\left(\|.\|_{v}\right)_{v \in \mathcal{P}_{K}}$ ainsi définie est une métrique adélique sur $\mathcal{L}$.

Une hauteur d'Arakelov sur $V$ est un couple $\left(\mathcal{L},\left(\|.\|_{v}\right)\right)$ où $\mathcal{L}$ est un fibré en droites sur $V$ et $\left(\|.\|_{v}\right)$ une métrique adélique sur $\mathcal{L}$.

Soit $\left(\mathcal{L},\left(\|.\|_{v}\right)\right)$ une hauteur d'Arakelov sur $V$. Soit $x \in V(K)$ et $s$ une section locale de $\mathcal{L}$ qui ne s'annule pas en $x$. Presque tous les facteurs du produit infini

$$
\prod_{v \in \mathcal{P}_{K}}\|s(x)\|_{v, x}^{-1}
$$


sont égaux à 1 ; le produit infini converge donc et sa valeur ne dépend pas du choix de $s$, d'après la formule du produit. On la note $H_{\mathcal{L}}(x)$ : c'est la hauteur (exponentielle) du point $x$ associée à la hauteur d'Arakelov $\left(\mathcal{L},\left(\|.\|_{v}\right)\right)$.

Soit $V$ une variété algébrique projective et lisse de dimension $d$ définie sur $K$, et $\left(\|.\|_{v}\right)_{v \in \mathcal{P}_{K}}$ une métrique adélique sur le faisceau anticanonique. On note $H$ la hauteur associée.

Pour tout $v \in \mathcal{P}_{K}$, une telle métrique définit une mesure $\omega_{V, v}$ sur $V\left(K_{v}\right)$ (on rappelle qu'on a choisi une normalisation de la mesure de Haar sur $K_{v}$, cf. la section 2.2).

Nous supposons à présent que que la variété $V$ vérifie les hypothèses suivantes (ce sont les hypothéses énoncées dans [Pe3, 2.1]) :

1. la classe du faisceau anticanonique de $V$ appartient à l'intérieur du cône effectif de $V$;

2. le groupe $\operatorname{Pic}\left(V^{\mathrm{s}}\right)$ (où $V^{\mathrm{s}}=V \times_{K} K^{\mathrm{s}}$ ) est un Z-module libre de rang fini, et coïncide avec $\operatorname{Pic}(\bar{V})$ (où $\left.\bar{V}=V \times_{K} \bar{K}\right)$;

3. les groupes de cohomologie $H^{1}\left(V, \mathcal{O}_{V}\right)$ et $H^{2}\left(V, \mathcal{O}_{V}\right)$ sont nuls ;

4. si $\ell$ est un nombre premier distinct de la caractéristique, la partie $p$ primaire de $\operatorname{Br}(\bar{V})$ est finie.

De telles hypothèses sont vérifiées en particulier par les variétés toriques projectives et lisses (cf. [Pe3, Remarque 2.1.1.] et [Pe2, Exemple 2.1.4]).

Pour des variétés vérifiant ces hypothèses, la mesure de Tamagawa sur l'espace adélique $V\left(\mathbf{A}_{K}\right)$ est donnée par la formule ${ }^{2}$

$$
\omega_{V}=c_{K, \operatorname{dim}(V)} \ell\left(\operatorname{Pic}\left(V^{\mathrm{s}}\right)\right) \prod_{v \in \mathcal{P}_{K}} L_{v}\left(1, \operatorname{Pic}\left(V^{\mathrm{s}}\right)\right)^{-1} \omega_{V, v}
$$

où l'on a posé, pour tout entier $d \geqslant 0$,

$$
c_{K, d}= \begin{cases}\operatorname{disc}(K)^{-\frac{d}{2}} & \text { dans le cas arithmétique, } \\ q_{K}^{\left(1-g_{K}\right) d} & \text { dans le cas fonctionnel. }\end{cases}
$$

Nous sommes à présent en mesure de définir la constante $C_{V, H}^{*}$ apparaissant dans les questions 1.4 et 1.6.

On définit en fait trois constantes à partir des données précédentes.

L'invariant $\alpha^{*}(V)$ est défini comme

$$
\alpha^{*}(V)=\int_{C_{\mathrm{eff}}(V)^{\vee}} e^{-\left\langle y, \omega_{V}^{-1}\right\rangle} d y
$$

\footnotetext{
${ }^{2}$ Un des points délicats de la construction est de montrer la convergence du produit; ceci nécessite d'une part la formule de Weil reliant volume $v$-adique et nombre de points sur le corps résiduel, d'autre part les conjectures de Weil prouvées par Deligne.
} 
où $C_{\text {eff }}(V) \subset \operatorname{Pic}(V)_{\mathbf{R}}$ est le cône effectif de $V$,

$$
C_{\text {eff }}(V)^{\vee}=\left\{y \in \operatorname{Pic}(V)_{\mathbf{R}}^{\vee}, \quad \forall x \in C_{\text {eff }}(V), \quad\langle y, x\rangle \geqslant 0\right\}
$$

et $d y$ est la mesure de Lebesgue sur $\operatorname{Pic}(V)_{\mathbf{R}}^{\vee}$ normalisée par le réseau $\operatorname{Pic}(V)^{\vee}$.

L'invariant $\beta(V)$ est défini comme

$$
\beta(V)=\left[H^{1}\left(K, \operatorname{Pic}\left(V^{\mathrm{s}}\right)\right)\right] .
$$

Enfin on définit

$$
\tau_{H}(V)=\omega_{V}(\overline{V(K)}),
$$

en d'autre termes $\tau_{H}(V)$ est le volume pour la mesure $\omega_{V}$ de l'adhérence de l'ensemble des points rationnels de $V$ dans l'espace adélique $V\left(\mathbf{A}_{K}\right)$.

La constante $C_{V, H}^{*}$ est alors égale par définition à $\frac{1}{(\operatorname{rg}(\operatorname{Pic}(V))-1) !} C_{V, H}$ où

$$
C_{V, H}=\alpha^{*}(V) \beta(V) \tau_{H}(V) .
$$

Soulignons que la nécessité d'introduire la constante $\beta(V)$ a été mise en évidence par le résultat de Batyrev et Tschinkel sur les variétés toriques.

\section{Tores algébriques}

\subsection{Quelques rappels}

Soit $K$ un corps. Un tore algébrique défini sur $K$ (de dimension $d$ ) est un groupe algébrique $T$ défini sur $K$ tel qu'il existe un isomorphisme de $\bar{K}$-groupes algébriques

$$
T_{\bar{K}} \stackrel{\sim}{\longrightarrow}\left(\mathbf{G}_{m, \bar{K}}\right)^{d} .
$$

Si $T$ est un tore algébrique défini sur $K$, on dit qu'une extension $L$ de $K$ déploie $T$ s'il existe un isomorphisme de $L$-groupes algébriques

$$
T_{L} \stackrel{\sim}{\longrightarrow}\left(\mathbf{G}_{m, L}\right)^{d} .
$$

Par [On2, Proposition 1.2.1], si $T$ est un tore algébrique défini sur $K$ il existe une extension séparable finie $L$ de $K$ qui déploie $T$. En particulier il existe une extension galoisienne finie $L$ de $K$ qui déploie $T$.

Soit $T$ un tore algébrique défini sur $K$ de dimension $d$. On note $X(T)$ le groupe des caractères de $T$, i.e. le groupe des morphismes de $K^{\mathrm{s}}$-groupes algébriques de $T_{K^{\text {s }}}$ vers $\mathbf{G}_{m, K^{\text {s }}}$. C'est un $\mathbf{Z}$-module libre de rang $d$, sur lequel le groupe $\operatorname{Gal}\left(K^{\mathrm{s}} / K\right)$ agit continûment, et qui dépend fonctoriellement de $T$. Si $L$ est une extension galoisienne de $K$ qui déploie $T$, l'action de $\operatorname{Gal}\left(K^{\mathrm{s}} / K\right)$ 
sur $X(T)$ se factorise à travers $\operatorname{Gal}(L / K)$. En outre le foncteur qui à un tore algébrique $T$ associe $X(T)$ définit une équivalence entre la catégorie des tores algébriques définis sur $K$ et la catégorie des Z-modules libres de rang fini muni d'une action continue de $\operatorname{Gal}\left(K^{\mathrm{s}} / K\right)$. Si $L / K$ est un extension finie galoisienne, cette équivalence induit une équivalence entre la catégorie des tores algébriques défini sur $K$ et déployés par $L$ et la catégorie des $\operatorname{Gal}(L / K)$ modules qui sont libres de rang fini comme $\mathbf{Z}$-modules.

Soit $T$ un tore algébrique défini sur $K$ et déployé par une extension finie galoisienne $L$ de $K$, de groupe de Galois $G$. Alors pour toute $K$-algèbre $K^{\prime}$ le groupe $T\left(K^{\prime}\right)$ des $K^{\prime}$-points de $T$ s'identifie canoniquement à

$$
\operatorname{Hom}_{G}\left(X(T),\left(L \otimes_{K} K^{\prime}\right)^{\times}\right)=\left(X(T)^{\vee} \otimes\left(L \otimes_{K} K^{\prime}\right)^{\times}\right)^{G} .
$$

En particulier, le groupe $T(K)$ des points $K$-rationnels de $T$ s'identifie canoniquement à

$$
\operatorname{Hom}_{G}\left(X(T), L^{\times}\right)=\left(X(T)^{\vee} \otimes L^{\times}\right)^{G} .
$$

Exemples 3.1: Un exemple immédiat de tore algébrique est fourni par les tores déployés, c'est-à-dire les groupes algébriques $K$-isomorphes à un produit de copies de $\mathbf{G}_{m, K}$.

Un autre exemple, important pour la suite, est donné par la situation suivante : soit $K_{0} / K$ une extension finie séparable, et $L / K$ une extension finie galoisienne de groupe $G$ contenant $K_{0}$. Soit $G_{0}$ le groupe de Galois de $L / K_{0}$. Au $G$-module $\mathbf{Z}\left[G / G_{0}\right]$ correspond, par l'équivalence de catégories cidessus, la restriction à la Weil de $K_{0}$ à $K$ de $\mathbf{G}_{m}$, notée $\operatorname{Res}_{K_{0} / K} \mathbf{G}_{m}$. En particulier on a la propriété

$$
\left(\operatorname{Res}_{K_{0} / K} \mathbf{G}_{m}\right)(K)=\left(K_{0}\right)^{\times} .
$$

Un tore algébrique sur $K$ est dit quasi-déployé s'il est isomorphe sur $K$ à un produit de tores du type $\operatorname{Res}_{K_{0} / K} \mathbf{G}_{m}$. Un tore algébrique sur $K$ (respectivement un tore algébrique sur $K$ déployé par une extension galoisienne finie de groupe $G$ ) est quasi-déployé si et seulement si son groupe des caractères est un $\operatorname{Gal}\left(K^{\mathrm{s}} / K\right)$-module de permutation (respectivement un $G$-module de permutation), c'est-à-dire possède une Z Z-base stable sous l'action de $\operatorname{Gal}\left(K^{\mathrm{s}} / K\right)$ respectivement $G$ ).

En fait dans la situation ci-dessus, pour tout tore algébrique $T$ défini sur $K_{0}$, on peut définir la restriction à la Weil de $K_{0}$ à $K$ de $T$ (cf. [On2, §1.4]), qui est un tore algébrique défini sur $K$ vérifiant en particulier

$$
\left(\operatorname{Res}_{K_{0} / K} T\right)(K)=T\left(K_{0}\right) .
$$

Par la suite seul le cas $T=\mathbf{G}_{m}$ nous sera utile. 


\section{Notations 3.2}

Soit $f: T_{1} \rightarrow T_{2}$ un morphisme de $K$-tores algébriques. Le morphisme de $\operatorname{Gal}\left(K^{\mathrm{s}} / K\right)$-modules $X\left(T_{2}\right) \rightarrow X\left(T_{1}\right)$ induit fonctoriellement par $f$ sera alors encore noté $f$. Le morphisme de groupes $T_{1}(K) \rightarrow T_{2}(K)$ induit par $f$ sera noté $f_{K}$.

\section{Lemme 3.3}

Soit $G$ un groupe fini et $M$ un $\mathbf{Z}$-module libre de rang fini, muni d'une action de $G$. Il existe une suite exacte de $G$-modules

$$
0 \rightarrow M \rightarrow P \rightarrow Q \rightarrow 0
$$

où $P$ et $Q$ sont libres de rang fini en tant que $\boldsymbol{Z}$-modules, et $P$ est un $G$ module de permutation.

Remarque 3.4: Ce lemme élémentaire nous sera utile dans la preuve des propositions 3.17 et 3.24 . Une version plus fine sera utilisée dans la section 3.8 .

Démonstration : Le dual $P^{\vee}$ d'un $G$-module de permutation $P$ étant encore un $G$-module de permutation, il suffit de construire un morphisme de $G$-modules surjectif $P^{\prime} \rightarrow M^{\vee}$, avec $P^{\prime}$ un $G$-module de permutation. Ceci peut se faire de la manière suivante : soit $\left(e_{1}, \ldots, e_{d}\right)$ une $\mathbf{Z}$-base de $M^{\vee}$ et pour $i=1, \ldots, d, G_{i}$ le stabilisateur de $e_{i}$ pour l'action de $G$. Pour $i=$ $1, \ldots, d$, il existe alors un unique morphisme de $G$-module $\mathbf{Z}\left[G / G_{i}\right] \rightarrow M^{\vee}$ envoyant $G_{i}$ sur $e_{i}$. Le morphisme somme $\oplus_{i=1}^{d} \mathbf{Z}\left[G / G_{i}\right] \rightarrow M^{\vee}$ répond à la question.

\subsection{L'espace adélique associé à un tore algébrique}

Soit $K$ un corps global. On note $\mathbf{G}_{m}\left(\mathbf{A}_{K}\right)$ le groupe des idèles de $K$, muni de la topologie adélique classique, qui en fait un groupe topologique abélien localement compact. L'injection diagonale $\mathbf{G}_{m}(K) \hookrightarrow \mathbf{G}_{m}\left(\mathbf{A}_{K}\right)$ identifie $\mathbf{G}_{m}(K)$ à un sous-groupe discret de $\mathbf{G}_{m}\left(\mathbf{A}_{K}\right)$.

Pour tout $v \in \mathcal{P}_{K}$, on note $\mathbf{G}_{m}\left(\mathcal{O}_{v}\right)=\mathcal{O}_{v}^{\times}$le sous-groupe compact maximal de $\mathbf{G}_{m}\left(K_{v}\right)=K_{v}^{\times}$. Soit

$$
\mathbf{K}\left(\mathbf{G}_{m}\right)=\prod_{v \in \mathcal{P}_{K}} \mathbf{G}_{m}\left(\mathcal{O}_{v}\right)
$$

c'est le sous-groupe compact maximal de $\mathbf{G}_{m}\left(\mathbf{A}_{K}\right)$.

Si $L / K$ est une extension finie, $\mathbf{G}_{m}\left(\mathbf{A}_{K}\right)$ s'injecte naturellement dans $\mathbf{G}_{m}\left(\mathbf{A}_{L}\right)$. Si de plus $L / K$ est galoisienne de groupe $G$, on a une action naturelle de $G$ sur $\mathbf{G}_{m}\left(\mathbf{A}_{L}\right)$ et alors

$$
\mathbf{G}_{m}\left(\mathbf{A}_{L}\right)^{G}=\mathbf{G}_{m}\left(\mathbf{A}_{K}\right) .
$$


On note $C_{K}=\mathbf{G}_{m}\left(\mathbf{A}_{K}\right) / \mathbf{G}_{m}(K)$ le groupe des classes d'idèles de $K$.

Nous décrivons maintenant la généralisation de ces notions à un tore algébrique $T$ quelconque. Bien entendu pour $T=\mathbf{G}_{m}$ on retrouvera les définitions précédentes. La construction de l'espace adélique associé à un tore algébrique $T$ est en fait un cas particulier de la construction générale de l'espace adélique associé à une variété algébrique définie sur $K$ (cf. [We2, I.2] et [Oe, I.3]). Elle peut se faire de la façon suivante.

Pour toute place $v$ de $K, T\left(K_{v}\right)$ est muni naturellement d'une structure de groupe topologique abélien localement compact. On désigne alors (abusivement) par $T\left(\mathcal{O}_{v}\right)$ le sous-groupe compact maximal de $T\left(K_{v}\right)$. En fait, si $S$ désigne l'ensemble des places de $K$ archimédiennes ou ramifiées dans une extension galoisienne $L$ de groupe $G$ déployant $T$ et $S_{L}$ l'ensemble des places de $L$ divisant une place de $S$, le schéma en groupes

$$
\mathcal{T}=\operatorname{Spec}\left(\mathcal{O}_{S_{L}} \otimes X(T)\right)^{G}
$$

est un modèle de $T$ sur $\operatorname{Spec}\left(\mathcal{O}_{S}\right)$, et pour toutes les places $v$ en dehors de $S$ on a $T\left(\mathcal{O}_{v}\right)=\mathcal{T}\left(\mathcal{O}_{v}\right)$, d'où la notation adoptée.

L'espace adélique associé à $T$ est alors le sous-groupe du groupe produit $\prod_{v} T\left(K_{v}\right)$ décrit par

$$
T\left(\mathbf{A}_{K}\right)=\left\{\left(t_{v}\right) \in \prod_{v} T\left(K_{v}\right), t_{v} \in T\left(\mathcal{O}_{v}\right) \text { pour presque tout } v \in \mathcal{P}_{K}\right\} .
$$

Pour un sous-ensemble fini $S$ de $\mathcal{P}_{K}$, on considérera aussi $T\left(\mathbf{A}_{K}\right)_{S}$ le sousgroupe de $\prod_{v} T\left(K_{v}\right)$ décrit par

$$
T\left(\mathbf{A}_{K}\right)_{S}=\left\{\left(t_{v}\right) \in \prod_{v} T\left(K_{v}\right), \forall v \notin S, t_{v} \in T\left(\mathcal{O}_{v}\right)\right\},
$$

de sorte que $T\left(\mathbf{A}_{K}\right)$ est la réunion des $T\left(\mathbf{A}_{K}\right)_{S}$ pour $S$ décrivant l'ensemble des parties finies de $\mathcal{P}_{K}$.

Comme sous-groupe de $\prod_{v} T\left(K_{v}\right), T\left(\mathbf{A}_{K}\right)$ est un groupe abélien. On le munit de la topologie dont une base d'ouverts est donnée par les sous-ensembles du type

$$
\prod_{v \in S} U_{v} \times \prod_{v \notin S} T\left(\mathcal{O}_{v}\right)
$$

où $S$ est un ensemble fini de places de $K$ et, pour $v \in S, U_{v}$ est un ouvert de $T\left(K_{v}\right)$. Cette topologie, qui est plus fine que la topologie issue de la topologie produit sur $\prod T\left(K_{v}\right)$, fait de $T\left(\mathbf{A}_{K}\right)$ un groupe topologique localement compact. On peut alors identifier $T(K)$ à un sous-groupe discret de $T\left(\mathbf{A}_{K}\right)$. 
On note

$$
\mathbf{K}(T)=T\left(\mathbf{A}_{K}\right)_{\varnothing}=\prod_{v \in \mathcal{P}_{K}} T\left(\mathcal{O}_{v}\right)
$$

c'est le sous-groupe compact maximal de $T\left(\mathbf{A}_{K}\right)$.

Si $L$ est une extension finie galoisienne de $K$ déployant $T$, de groupe de Galois $G$, on dispose également, comme pour $T(K)$, d'une description simple de tous ces groupes en termes du $G$-module $X(T)$, pratique pour manipuler des suites exactes. Pour toute place $v$ de $K$, on a d'après (3.3) une identification canonique

$$
T\left(K_{v}\right) \stackrel{\sim}{\longrightarrow} \operatorname{Hom}_{G}\left(X(T),\left(L \otimes_{K} K_{v}\right)^{\times}\right) .
$$

Rappelons que $\left(L \otimes_{K} K_{v}\right)^{\times}$s'identifie à $\prod_{\mathcal{V} \mid v} L_{\mathcal{V}}^{\times}$. L'identification (3.8) induit une identification

$$
T\left(\mathcal{O}_{v}\right) \stackrel{\sim}{\longrightarrow} \operatorname{Hom}_{G}\left(X(T), \prod_{\mathcal{V} \mid v} \mathcal{O}_{\mathcal{V}}^{\times}\right)
$$

Si on choisit une place $\mathcal{V}$ divisant $v$ et si on note $G_{v}$ son groupe de décomposition, les identifications (3.8) et (3.9) induisent respectivement des identifications

$$
T\left(K_{v}\right) \stackrel{\sim}{\longrightarrow} \operatorname{Hom}_{G_{v}}\left(X(T), L_{\mathcal{V}}^{\times}\right)
$$

et

$$
T\left(\mathcal{O}_{v}\right) \stackrel{\sim}{\longrightarrow} \operatorname{Hom}_{G_{v}}\left(X(T), \mathcal{O}_{\mathcal{V}}^{\times}\right) .
$$

On peut identifier $T\left(\mathbf{A}_{K}\right)$ au groupe

$$
\operatorname{Hom}_{G}\left(X(T), \mathbf{G}_{m}\left(\mathbf{A}_{L}\right)\right)
$$

muni de la topologie induite par celle de $\mathbf{G}_{m}\left(\mathbf{A}_{L}\right)$, et $\mathbf{K}(T)$ au groupe

$$
\operatorname{Hom}_{G}\left(X(T), \mathbf{K}\left(\mathbf{G}_{m, L}\right)\right) \text {. }
$$

Exemple 3.5: Soit $K_{0}$ une extension finie séparable de $K$, et $T=\operatorname{Res}_{K_{0} / K} \mathbf{G}_{m}$. Alors $T\left(\mathbf{A}_{K}\right)$ s'identifie canoniquement à $\mathbf{G}_{m}\left(\mathbf{A}_{K_{0}}\right)$.

\section{Notations 3.6}

Soit $f: T_{1} \rightarrow T_{2}$ un morphisme $K$-de tores algébriques, et $v$ une place de $K$. Le morphisme continu de groupes topologiques $T_{2}\left(\boldsymbol{A}_{K}\right) \rightarrow T_{1}\left(\boldsymbol{A}_{K}\right)$ (respectivement $T_{2}\left(K_{v}\right) \rightarrow T_{1}\left(K_{v}\right)$ ) induit fonctoriellement par $f$ sera alors noté $f_{\boldsymbol{A}_{K}}$ (respectivement $f_{v}$ ). 


\subsection{Le degré}

\subsubsection{Définitions}

Soit $K$ un corps global. Pour toute place $v$ de $K$, on a un morphisme de valuation défini par

$$
\begin{aligned}
\operatorname{deg}_{K, v}: \mathbf{G}_{m}\left(K_{v}\right) & \longrightarrow \mathbf{Z} \\
x_{v} & \longmapsto v\left(x_{v}\right)
\end{aligned}
$$

si $v$ est finie et

$$
\begin{aligned}
\operatorname{deg}_{K, v}: \mathbf{G}_{m}\left(K_{v}\right) & \longrightarrow \mathbf{R} \\
x_{v} & \longmapsto \log \left|x_{v}\right|_{v}
\end{aligned}
$$

si $v$ est archimédienne. Dans les deux cas, ce morphisme a pour noyau $\mathbf{G}_{m}\left(\mathcal{O}_{v}\right)$

On en déduit un morphisme «degré» défini par

$$
\begin{aligned}
\operatorname{deg}_{K}: \mathbf{G}_{m}\left(\mathbf{A}_{K}\right) & \longrightarrow \mathbf{R} \\
\left(x_{v}\right) & \longmapsto \sum_{v \in \mathcal{P}_{K}} \log \left(q_{v}\right) \operatorname{deg}_{K, v}\left(x_{v}\right)
\end{aligned}
$$

dans le cas arithmétique et

$$
\begin{aligned}
\operatorname{deg}_{K}: \mathbf{G}_{m}\left(\mathbf{A}_{K}\right) & \longrightarrow \mathbf{Z} \\
\left(x_{v}\right) & \longmapsto \sum_{v \in \mathcal{P}_{K}} f_{v} \operatorname{deg}_{K, v}\left(x_{v}\right) .
\end{aligned}
$$

Dans le cas arithmétique, le morphisme $\operatorname{deg}_{K}$ est surjectif, car le morphisme $\operatorname{deg}_{K, v}$ est surjectif si $v$ est archimédienne. Dans le cas fonctionnel, $\operatorname{deg}_{K}$ est surjectif d'après [We1, VII $\S 5$, Cor 6].

Dans les deux cas, le noyau de $\operatorname{deg}_{K}$ contient $\mathbf{G}_{m}(K)$ (par la formule du produit) et $\mathbf{K}\left(\mathbf{G}_{m}\right)$. Il sera noté $\mathbf{G}_{m}\left(\mathbf{A}_{K}\right)^{1}$.

Soit $L$ une extension finie de $K$. Dans le cas arithmétique, on a la relation

$$
\operatorname{deg}_{\left.L\right|_{\mathbf{G}_{m}\left(\mathbf{A}_{K}\right)}}=[L: K] \operatorname{deg}_{K} .
$$

Dans le cas fonctionnel, on a la relation

$$
d_{L} \operatorname{deg}_{\left.L\right|_{\mathbf{G}_{m}\left(\mathbf{A}_{K}\right)}}=d_{K}[L: K] \operatorname{deg}_{K},
$$

où l'on rappelle que $q^{d_{L}}$ et $q^{d_{K}}$ représentent les cardinaux des corps des constantes des corps de fonctions $L$ et $K$ respectivement.

Soit à présent $T$ un tore algébrique défini sur $K$, déployé par une extension finie galoisienne $L$ de groupe de Galois $G$. Soit $m$ un élément de 
$X(T)^{G}$, c'est-à-dire un morphisme de $K$-groupes $T \rightarrow \mathbf{G}_{m}$ (le morphisme dual est alors le morphisme de $G$-modules $\mathbf{Z} \rightarrow X(T)$ qui envoie 1 sur $m$ ).

Par composition du morphisme continu $m_{\mathbf{A}_{K}}: T\left(\mathbf{A}_{K}\right) \rightarrow \mathbf{G}_{m}\left(\mathbf{A}_{K}\right)$ avec $\operatorname{deg}_{K}$ on obtient un morphisme continu $T\left(\mathbf{A}_{K}\right) \rightarrow \mathbf{R}$ dans le cas arithmétique et $T\left(\mathbf{A}_{K}\right) \rightarrow \mathbf{Z}$ dans le cas fonctionnel. On note $\operatorname{deg}_{T}$ le morphisme défini par

$$
\begin{aligned}
T\left(\mathbf{A}_{K}\right) & \longrightarrow \operatorname{Hom}\left(X(T)^{G}, \mathbf{R}\right) \\
t & \longmapsto\left[m \mapsto\left(\operatorname{deg}_{K} \circ m_{\mathbf{A}_{K}}\right)(t)\right]
\end{aligned}
$$

dans le cas arithmétique, et

$$
\begin{aligned}
T\left(\mathbf{A}_{K}\right) & \longrightarrow \operatorname{Hom}\left(X(T)^{G}, \mathbf{Z}\right) \\
t & \longmapsto\left[m \mapsto\left(\operatorname{deg}_{K} \circ m_{\mathbf{A}_{K}}\right)(t)\right]
\end{aligned}
$$

dans le cas fonctionnel. Dans ce dernier cas, le morphisme deg $_{T}$ n'est autre que le morphisme $\theta$ défini par Oesterlé dans [Oe, I.5.5], composé avec $\log _{q_{K}}$.

Dans les deux cas, on a $\operatorname{deg}_{\mathbf{G}_{m}}=\operatorname{deg}_{K}$. Le résultat suivant est immédiat.

\section{Lemme 3.7}

Le morphisme $\operatorname{deg}_{T}$ est fonctoriel dans le sens suivant : soit

$$
f: T_{1} \longrightarrow T_{2}
$$

est un morphisme de $K$-tores algébriques déployés par L. Le morphisme

$$
f: X\left(T_{2}\right) \longrightarrow X\left(T_{1}\right)
$$

de G-module associé induit par dualité des morphismes

$$
f^{\vee}:\left(X\left(T_{1}\right)^{G}\right)^{\vee} \longrightarrow\left(X\left(T_{2}\right)^{G}\right)^{\vee}
$$

et

$$
f_{\mathbf{R}}^{\vee}:\left(X\left(T_{1}\right)^{G}\right)_{\mathbf{R}}^{\vee} \longrightarrow\left(X\left(T_{2}\right)^{G}\right)_{\mathbf{R}}^{\vee}
$$

On a alors

$$
\operatorname{deg}_{T_{2}} \circ f_{\boldsymbol{A}_{K}}=f_{\boldsymbol{R}}^{\vee} \circ \operatorname{deg}_{T_{1}}
$$

dans le cas arithmétique et

$$
\operatorname{deg}_{T_{2}} \circ f_{\boldsymbol{A}_{K}}=f^{\vee} \circ \operatorname{deg}_{T_{1}}
$$

dans le cas fonctionnel. 
Pour un tore algébrique $T$ quelconque, à l'instar $\operatorname{de} \operatorname{deg}_{K}$, $\operatorname{deg}_{T}$ se décompose en une somme de degrés locaux, que nous décrivons à présent. Soit $v$ une place de $K, G_{v}$ un groupe de décomposition au-dessus de $v$ et $m$ un élément de $X(T)^{G_{v}}$, c'est-à-dire un morphisme de $K_{v}$-tores $T_{K_{v}} \rightarrow \mathbf{G}_{m, K_{v}}$. Par composition du morphisme continu $m_{v}: T\left(K_{v}\right) \rightarrow \mathbf{G}_{m}\left(K_{v}\right)$ avec $\operatorname{deg}_{K, v}$ on obtient un morphisme continu $T\left(K_{v}\right) \rightarrow \mathbf{Z}$ si $v$ est finie et $T\left(K_{v}\right) \rightarrow \mathbf{R}$ si $v$ est archimédienne. On note $\operatorname{deg}_{T, v}$ le morphisme défini par

$$
\begin{aligned}
T\left(K_{v}\right) & \longrightarrow \operatorname{Hom}\left(X(T)^{G_{v}}, \mathbf{Z}\right) \\
t & \longmapsto\left[m \mapsto\left(\operatorname{deg}_{K, v} \circ m_{v}\right)(t)\right]
\end{aligned}
$$

si $v$ est finie et

$$
\begin{aligned}
T\left(K_{v}\right) & \longrightarrow \operatorname{Hom}\left(X(T)^{G_{v}}, \mathbf{R}\right) \\
t & \longmapsto\left[m \mapsto\left(\operatorname{deg}_{K, v} \circ m_{v}\right)(t)\right]
\end{aligned}
$$

si $v$ est archimédienne. Dans les deux cas, le noyau de $\operatorname{deg}_{T, v}$ est $T\left(\mathcal{O}_{v}\right)$.

Notons $i_{T, v}$ l'injection continue naturelle de groupes topologiques $T\left(K_{v}\right) \rightarrow$ $T\left(\mathbf{A}_{K}\right)$.

Dans le cas arithmétique, pour toute place $v$, le diagramme

$$
\begin{gathered}
T\left(K_{v}\right) \stackrel{i_{T, v}}{\longrightarrow} T\left(\mathbf{A}_{K}\right) \\
\downarrow^{\log \left(q_{v}\right) \operatorname{deg}_{T, v}} \quad \\
\operatorname{Hom}\left(X(T)^{G_{v}}, \mathbf{R}\right) \longrightarrow \operatorname{Hom}\left(X(T)^{G}, \mathbf{R}\right)
\end{gathered}
$$

(où la flèche horizontale du bas est le morphisme naturel de restriction) est commutatif, et on a

$$
\operatorname{deg}_{T}=\sum_{v \in \mathcal{P}_{K}} \log \left(q_{v}\right) \operatorname{deg}_{T, v} .
$$

Dans le cas fonctionnel, pour toute place $v$ le diagramme

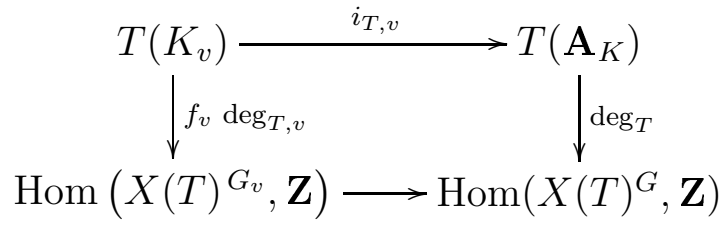

(où la flèche horizontale du bas est le morphisme naturel de restriction) est commutatif, et on a

$$
\operatorname{deg}_{T}=\sum_{v \in \mathcal{P}_{K}} f_{v} \operatorname{deg}_{T, v}
$$


Le noyau de $\operatorname{deg}_{T}$ sera noté $T\left(\mathbf{A}_{K}\right)^{1}$. Par la formule du produit, $T(K)$ est contenu dans $T\left(\mathbf{A}_{K}\right)^{1}$. Par ailleurs, comme chaque morphisme $\operatorname{deg}_{T, v}$ a pour noyau $T\left(\mathcal{O}_{v}\right), T\left(\mathbf{A}_{K}\right)^{1}$ contient $\mathbf{K}(T)$. En outre, $T\left(\mathbf{A}_{K}\right)^{1}$ s'identifie au $^{\prime}$ groupe

$$
T\left(\mathbf{A}_{K}\right)^{1}=\operatorname{Hom}_{G}\left(X(T), \mathbf{G}_{m}\left(\mathbf{A}_{L}\right)^{1}\right) .
$$

Pour toute partie finie $S$ de $\mathcal{P}_{K}$, on note

$$
T\left(\mathbf{A}_{K}\right)_{S}^{1}=T\left(\mathbf{A}_{K}\right)_{S} \cap T\left(\mathbf{A}_{K}\right)^{1} .
$$

On définit à présent une variante du morphisme $\operatorname{deg}_{T}$ et des degrés locaux $\operatorname{deg}_{T, v}$ qui nous sera utile par la suite. Contrairement à $\operatorname{deg}_{T}$, la définition de cette variante dépend du choix de l'extension $L$ déployant $T$. Pour comparer les deux notions, nous aurons besoin du lemme élémentaire suivant.

\section{Lemme 3.8}

Soit $M$ un $G$-module qui est un $\mathbf{Z}$-module libre de rang fini. Alors la flèche naturelle

$$
\left(M^{\vee}\right)^{G} \longrightarrow\left(M^{G}\right)^{\vee}
$$

est une injection de conoyau fini.

Démonstration: Le quotient $M / M^{G}$ étant sans torsion, on a une suite exacte

$$
0 \rightarrow\left(M / M^{G}\right)^{\vee} \rightarrow M^{\vee} \rightarrow\left(M^{G}\right)^{\vee} \rightarrow 0
$$

En prenant les $G$-invariants, on obtient la suite exacte

$$
0 \rightarrow\left(\left(M / M^{G}\right)^{\vee}\right)^{G} \rightarrow\left(M^{\vee}\right)^{G} \rightarrow\left(M^{G}\right)^{\vee} \rightarrow H^{1}\left(G,\left(M / M^{G}\right)^{\vee}\right)
$$

dont l'avant-dernière flèche est la flèche de l'énoncé. Son conoyau est donc fini. Soit $\phi$ un élément de $\left(M^{\vee}\right)^{G}=\operatorname{Hom}_{G}(M, \mathbf{Z})$ dont la restriction à $M^{G}$ est nulle. Pour tout $x \in M, N_{G} x \stackrel{\text { deff }}{=} \sum_{g \in G} g . x$ est un élément de $M^{G}$ et on a

$$
0=\phi\left(N_{G} x\right)=[G] \phi(x)
$$

donc $\phi(x)=0$. Ainsi $\phi$ est nulle, d'où l'injectivité (en d'autres termes, le dual d'un $G$-module anisotrope est anisotrope).

On se place dans le cas arithmétique. On considère la suite exacte

$$
0 \longrightarrow \mathbf{G}_{m}\left(\mathbf{A}_{L}\right)^{1} \longrightarrow \mathbf{G}_{m}\left(\mathbf{A}_{L}\right) \stackrel{\operatorname{deg}_{L}}{\longrightarrow} \mathbf{R} \longrightarrow 0 .
$$

Tensorisons par $X(T)^{\vee}$ et prenons les $G$-invariants. On obtient la suite exacte

$$
0 \longrightarrow T\left(\mathbf{A}_{K}\right)^{1} \longrightarrow T\left(\mathbf{A}_{K}\right) \longrightarrow\left(X(T)^{\vee}\right)_{\mathbf{R}}^{G}
$$


et donc un morphisme

$$
\operatorname{deg}_{T, L}: T\left(\mathbf{A}_{K}\right) \longrightarrow\left(X(T)^{\vee}\right)^{G},
$$

de noyau $T\left(\mathbf{A}_{K}\right)^{1}$. On notera encore $\operatorname{deg}_{T, L}$ le morphisme obtenu par composition avec le morphisme de restriction $\left(X(T)^{\vee}\right)_{\mathbf{R}}^{G} \hookrightarrow\left(X(T)^{G}\right)_{\mathbf{R}}^{\vee}$ (qui est un isomorphisme d'après le lemme 3.8), On a donc, pour tout $t \in T\left(\mathbf{A}_{K}\right)$ et tout $m \in X(T)^{G}$,

$$
\left\langle\operatorname{deg}_{T, L}(x), m\right\rangle=\operatorname{deg}_{L}\left(m_{\mathbf{A}_{K}}(t)\right) .
$$

D'après (3.5) on a donc

$$
\operatorname{deg}_{T, L}=[L: K] \operatorname{deg}_{T}
$$

ce qui montre en particulier que contrairement au morphisme $\operatorname{deg}_{T}$, le morphisme $\operatorname{deg}_{T, L}$ dépend du choix de l'extension déployant $T$.

Remarquons que le diagramme

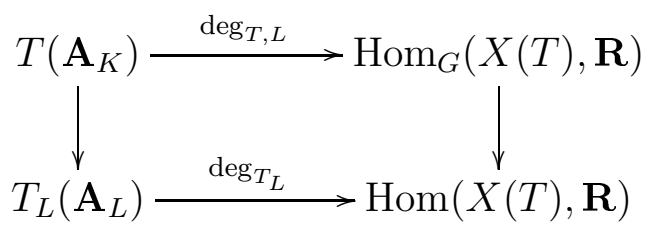

(où les flèches verticales sont les inclusions naturelles) est commutatif.

Plac̀ ;ons nous à présent dans le cas fonctionnel. On considère la suite exacte

$$
0 \longrightarrow \mathbf{G}_{m}\left(\mathbf{A}_{L}\right)^{1} \longrightarrow \mathbf{G}_{m}\left(\mathbf{A}_{L}\right) \stackrel{\operatorname{deg}_{L}}{\longrightarrow} \mathbf{Z} \longrightarrow 0 .
$$

Tensorisons par $X(T)^{\vee}$ et prenons les $G$-invariants. On obtient la suite exacte

$$
0 \longrightarrow T\left(\mathbf{A}_{K}\right)^{1} \longrightarrow T\left(\mathbf{A}_{K}\right) \longrightarrow\left(X(T)^{\vee}\right)^{G}
$$

et donc un morphisme

$$
\operatorname{deg}_{T, L}: T\left(\mathbf{A}_{K}\right) \longrightarrow\left(X(T)^{\vee}\right)^{G},
$$

de noyau $T\left(\mathbf{A}_{K}\right)^{1}$. On notera encore $\operatorname{deg}_{T, L}$ le morphisme obtenu par composition avec le morphisme de restriction $\left(X(T)^{\vee}\right)^{G} \hookrightarrow\left(X(T)^{G}\right)^{\vee}$ (qui est injectif d'après le lemme 3.8), On a donc, pour tout $t \in T\left(\mathbf{A}_{K}\right)$ et tout $m \in X(T)^{G}$,

$$
\left\langle\operatorname{deg}_{T, L}(t), m\right\rangle=\operatorname{deg}_{L}\left(m_{\mathbf{A}_{K}}(t)\right) .
$$


D'après (3.6), on a donc la relation

$$
d_{L} \operatorname{deg}_{T, L}=d_{K}[L: K] \operatorname{deg}_{T}
$$

ce qui montre que contrairement au morphisme $\operatorname{deg}_{T}$, le morphisme $\operatorname{deg}_{T, L}$ dépend du choix de l'extension déployant $T$.

Remarquons que le diagramme

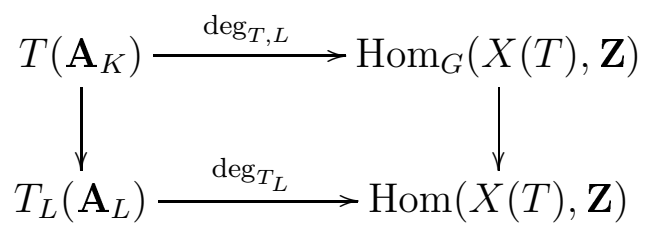

(où les flèches verticales sont les inclusions naturelles) est commutatif.

Tout comme le morphisme $\operatorname{deg}_{T}$, le morphisme $\operatorname{deg}_{T, L}$ peut se décomposer (de manière non canonique) en une somme de degrés locaux, que nous décrivons à présent.

Soit $v$ une place finie de $K, v$ une place de $L$ divisant $v$ et $G_{v}$ son groupe de décomposition. On considère la suite exacte de $G_{v}$-modules.

$$
0 \longrightarrow \mathcal{O}_{\mathcal{V}}^{\times} \longrightarrow K_{\mathcal{V}}^{\times} \stackrel{\mathcal{V}}{\longrightarrow} \mathbf{Z} \longrightarrow 0 .
$$

Tensorisons par $X(T)^{\vee}$ et prenons les $G_{v}$-invariants. On obtient la suite exacte

$$
0 \longrightarrow T\left(\mathcal{O}_{v}\right) \longrightarrow T\left(K_{v}\right) \longrightarrow\left(X(T)^{\vee}\right)^{G_{v}}
$$

et donc un morphisme

$$
\operatorname{deg}_{T, L, \mathcal{V}}: T\left(K_{v}\right) \longrightarrow\left(X(T)^{\vee}\right)^{G_{v}}
$$

de noyau $T\left(\mathcal{O}_{v}\right)$. Remarquons que le diagramme

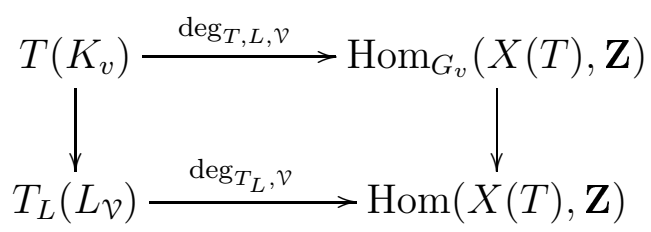

(où les flèches verticales sont les inclusions naturelles) est commutatif.

Soit à présent $v$ une place archimédienne, $V$ une place de $L$ divisant $v$ et $G_{v}$ son groupe de décomposition. On considère la suite exacte de $G_{v^{-}}$modules.

$$
0 \longrightarrow \mathcal{O}_{\mathcal{V}}^{\times} \longrightarrow K_{\mathcal{V}}^{\times} \stackrel{\log |\cdot|_{v}}{\longrightarrow} \mathbf{R} \longrightarrow 0 .
$$


Tensorisons par $X(T)^{\vee}$ et prenons les $G_{v}$-invariants. On obtient la suite exacte

$$
0 \longrightarrow T\left(\mathcal{O}_{v}\right) \longrightarrow T\left(K_{v}\right) \longrightarrow\left(X(T)^{\vee}\right)_{\mathbf{R}}^{G_{v}}
$$

et donc un morphisme

$$
\operatorname{deg}_{T, L, \mathcal{V}}: T\left(K_{v}\right) \longrightarrow\left(X(T)^{\vee}\right)_{\mathbf{R}}^{G_{v}}
$$

de noyau $T\left(\mathcal{O}_{v}\right)$. Remarquons que le diagramme

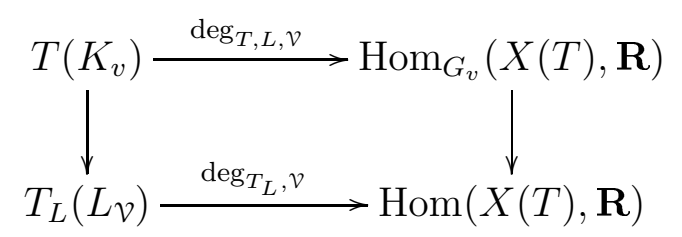

(où les flèches verticales sont les inclusions naturelles) est commutatif.

Dans le cas arithmétique, pour toute place $v$ le diagramme

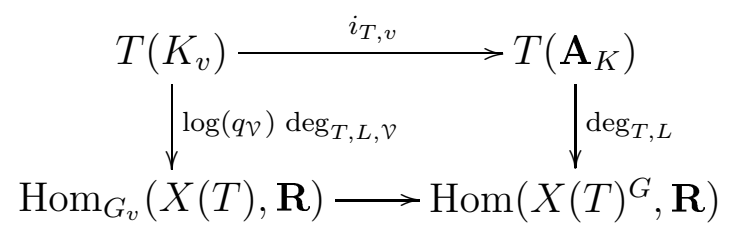

(où la flèche horizontale du bas est le morphisme de restriction) est commutatif, et on a

$$
\operatorname{deg}_{T, L}=\sum_{v \in \mathcal{P}_{K}} \log \left(q_{\mathcal{V}}\right) \operatorname{deg}_{T, L, \mathcal{V}} .
$$

Dans le cas fonctionnel, pour toute place $v$ le diagramme

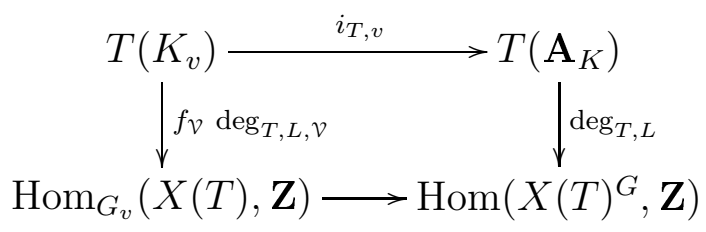

(où la flèche horizontale du bas est le morphisme de restriction) est commutatif et on a

$$
\operatorname{deg}_{T, L}=\sum_{v \in \mathcal{P}_{K}} f_{\mathcal{V}} \operatorname{deg}_{T, L, \mathcal{V}}
$$




\subsubsection{Propriétés du degré local dans le cas d'une place finie}

On considère toujours un tore algébrique $T$ défini sur un corps global $K$ et déployé par une extension galoisienne $L$ de groupe $G$

\section{Lemme 3.9}

Soit $v$ une place finie de $K$ non ramifiée dans $L, \mathcal{V}$ une place de $L$ divisant $v$ et $G_{v}$ le groupe de décomposition correspondant. Alors le morphisme

$$
\operatorname{deg}_{T, L, \mathcal{V}}: T\left(K_{v}\right) \longrightarrow\left(X(T)^{\vee}\right)^{G_{v}}
$$

est surjectif, et induit donc un isomorphisme

$$
T\left(K_{v}\right) / T\left(\mathcal{O}_{v}\right) \stackrel{\sim}{\longrightarrow}\left(X(T)^{\vee}\right)^{G_{v}} .
$$

Remarque 3.10 : On verra plus loin (cf. la proposition 3.27) que pour une place finie quelconque le morphisme (3.51) est de conoyau fini.

Démonstration : L'argument qui suit est repris de la page 449 de [Dr]. $\mathrm{Au}$ vu de la construction de $\operatorname{deg}_{T, L, \mathcal{v}}$, le conoyau du morphisme (3.51) est le groupe $H^{1}\left(G_{v}, X(T)^{\vee} \otimes \mathcal{O}_{\mathcal{V}}^{\times}\right)$. Or, si $v$ est non ramifiée, $\mathcal{O}_{\mathcal{V}}^{\times}$est cohomologiquement trivial, et comme $X(T)^{\vee}$ est sans torsion, d'après [Se1, IX, $\S 5$, Corollaire], $X(T)^{\vee} \otimes \mathcal{O}_{\mathcal{V}}^{\times}$est encore cohomologiquement trivial, d'où le résultat.

\section{Lemme 3.11}

Soit $v$ une place finie de $K, \mathcal{V}$ une place de $L$ divisant $v, G_{v}$ son groupe de décomposition et $e_{v}$ l'indice de ramification de $v$ dans $L$.

On a alors

$$
\operatorname{deg}_{T, L, \mathcal{V}}=e_{v} \operatorname{deg}_{T, v} .
$$

Démonstration : On a en effet, pour $t \in T\left(K_{v}\right)$, et pour $m \in X(T)^{G}$,

$$
\begin{aligned}
\left\langle\operatorname{deg}_{T, L, \mathcal{V}}(t), m\right\rangle & =\mathcal{V}\left(m_{v}(t)\right) \\
& =e_{v} v(\langle x, t\rangle) \\
& =e_{v}\left\langle\operatorname{deg}_{T, v}(t), m\right\rangle .
\end{aligned}
$$

\subsubsection{Propriétés du degré dans le cas arithmétique}

\section{Lemme 3.12}

Soit $v$ une place archimédienne de $K, \mathcal{V}$ une place de $L$ divisant $v, G_{v}$ son groupe de décomposition.

On a alors

$$
\operatorname{deg}_{T, L, \mathcal{V}}=\left[L_{\mathcal{V}}: K_{v}\right] \operatorname{deg}_{T, v}
$$


Démonstration : Soit $t \in T\left(K_{v}\right)$ et $m \in X(T)^{G}$. On a

$$
\begin{aligned}
\left\langle\operatorname{deg}_{T, L, \mathcal{V}}(x), t\right\rangle & =\log \left(\left|m_{v}(t)\right|_{\mathcal{V}}\right) \\
& =\left[L_{\mathcal{V}}: K_{v}\right] \log \left(\left|m_{v}(t)\right|_{v}\right) \\
& =\left[L_{\mathcal{V}}: K_{v}\right]\left\langle\operatorname{deg}_{T, v}(t), m\right\rangle .
\end{aligned}
$$

\section{Lemme 3.13}

Soit $v$ une place archimédienne de $K, V$ une place de $L$ divisant $v$ et $G_{v}$ son groupe de décomposition. Le morphisme

$$
\operatorname{deg}_{T, L, \mathcal{V}}: T\left(K_{v}\right) \longrightarrow \operatorname{Hom}_{G_{v}}(X(T), \boldsymbol{R})
$$

est surjectif.

En particulier le morphisme

$$
\operatorname{deg}_{T, L, \mathcal{V}}: T\left(K_{v}\right) \longrightarrow \operatorname{Hom}\left(X(T)^{G}, \boldsymbol{R}\right)
$$

est surjectif.

Démonstration : Le morphisme (3.61) est surjectif car il admet pour section le morphisme

$$
\operatorname{Hom}_{G_{v}}(X(T), \mathbf{R}) \longrightarrow \operatorname{Hom}_{G_{v}}\left(X(T), \mathbf{R}^{\times}\right) \subset \operatorname{Hom}_{G_{v}}\left(X(T), L_{\mathcal{V}}^{\times}\right) \stackrel{\sim}{\rightarrow} T\left(K_{v}\right)
$$

obtenu par composition soit avec l'exponentielle si $L_{\mathcal{V}}=\mathbf{R}$, soit avec le carré de l'exponentielle si $L_{V} \stackrel{\sim}{\rightarrow} \mathbf{C}$.

La deuxième assertion vient du fait que le morphisme de $\mathbf{R}$-espaces vectoriels

$$
\operatorname{Hom}_{G_{v}}(X(T), \mathbf{R}) \rightarrow \operatorname{Hom}\left(X(T)^{G_{v}}, \mathbf{R}\right)
$$

est un isomorphisme, et que le morphisme de restriction

$$
\operatorname{Hom}\left(X(T)^{G_{v}}, \mathbf{R}\right) \rightarrow \operatorname{Hom}\left(X(T)^{G}, \mathbf{R}\right)
$$

est surjectif.

\section{Corollaire 3.14}

Dans le cas arithmétique, $\operatorname{deg}_{T}$ et $\operatorname{deg}_{T, L}$ sont surjectifs.

Démonstration : Pour toute place $v$ archimédienne, on a d'après le lemme 3.13

$$
\operatorname{deg}_{T, L, \mathcal{V}}\left(T\left(K_{v}\right)\right)=\operatorname{Hom}\left(X(T)^{G}, \mathbf{R}\right) .
$$


ce qui montre la surjectivité de $\operatorname{deg}_{T, L}$ d'après (3.48).

Par ailleurs, pour toute place $v$ archimédienne, $T\left(K_{v}\right)$ est un groupe divisible, et $\operatorname{deg}_{T, v}$ et $\operatorname{deg}_{T, L, \mathcal{V}}$ sont proportionnels. On en déduit qu'on a

$$
\operatorname{deg}_{T, v}\left(T\left(K_{v}\right)\right)=\operatorname{Hom}\left(X(T)^{G}, \mathbf{R}\right) .
$$

ce qui montre la surjectivité de $\operatorname{deg}_{T}$ d'après (3.18).

\subsubsection{Propriétés du degré dans le cas fonctionnel}

Contrairement à ce qui se passe dans le cas arithmétique, $\operatorname{deg}_{T}$ n'est plus nécessairement surjectif dans le cas fonctionnel, comme le montre le lemme suivant.

\section{Lemme 3.15}

Soit $K$ un corps de fonctions et $L / K$ une extension finie galoisienne, de groupe de Galois $G$. Soit $K_{0}$ une extension de $K$ contenue dans $L, T$ le tore $\operatorname{Res}_{K_{0} / K} \boldsymbol{G}_{m}$ et $G_{0}=\operatorname{Gal}\left(L / K_{0}\right)$. Soit $d_{0}$ tel que $q_{K_{0}}=q_{K}^{d_{0}}$, de sorte que $d_{0}=\frac{d_{K_{0}}}{d_{K}}$. Alors, via les identifications naturelles $T\left(\boldsymbol{A}_{K}\right)=\boldsymbol{G}_{m}\left(\boldsymbol{A}_{K_{0}}\right)$ et $\left(X(T)^{G}\right)^{\vee}=\boldsymbol{Z}$, le morphisme $\operatorname{deg}_{T}$ n'est autre que le morphisme $d_{0} \operatorname{deg}_{K_{0}}$, et son image est $d_{0} \boldsymbol{Z}$. En particulier $\operatorname{deg}_{T}$ n'est pas nécessairement surjectif.

Démonstration : Soit $t \in \mathbf{G}_{m}\left(\mathbf{A}_{K_{0}}\right)$. Via l'identification

$$
T\left(\mathbf{A}_{K}\right) \stackrel{\sim}{\longrightarrow} \mathbf{G}_{m}\left(\mathbf{A}_{K_{0}}\right),
$$

il lui correspond l'élément de

$$
T\left(\mathbf{A}_{K}\right)=\operatorname{Hom}_{G}\left(\mathbf{Z}\left[G / G_{0}\right], \mathbf{G}_{m}\left(\mathbf{A}_{L}\right)\right)
$$

qui envoie $G_{0}$ sur $t$. Le $\mathbf{Z}$-module $\mathbf{Z}\left[G / G_{0}\right]^{G}$ est de rang 1 engendré par $\sum_{g \in G / G_{0}} g G_{0}$. Le morphisme $\operatorname{deg}_{T}(t)$ envoie alors $\sum_{g \in G / G_{0}} g G_{0}$ sur

$$
\begin{aligned}
\operatorname{deg}_{K}\left(\prod_{g \in G / G_{0}} g t\right) & =\frac{d_{L}}{d_{K}[L: K]} \sum_{g \in G / G_{0}} \operatorname{deg}_{L}(g t) \\
& =\frac{d_{L}[G]}{d_{K}[L: K]\left[G_{0}\right]} \operatorname{deg}_{L}(t) \\
& =\frac{d_{L}}{d_{K}\left[L: K_{0}\right]} \operatorname{deg}_{L}(t) \\
& =d_{0} \frac{d_{L}}{d_{K_{0}}\left[L: K_{0}\right]} \operatorname{deg}_{L}(t) \\
& =d_{0} \operatorname{deg}_{K_{0}}(t)
\end{aligned}
$$


la première et la dernière égalité venant de la formule (3.6). Le fait que l'image est $d_{0} \mathbf{Z}$ découle alors de l'existence de diviseurs de degré 1 ([We1, VII, §5,Cor 6]).

\section{Notation 3.16}

Dans le cas fonctionnel, on note $\mathcal{D}_{T}$ l'image du morphisme $\operatorname{deg}_{T}$.

\section{Proposition 3.17}

On se place dans le cas fonctionnel. Le morphisme

$$
\operatorname{deg}_{T, L}: T\left(\boldsymbol{A}_{K}\right) \longrightarrow\left(X(T)^{\vee}\right)^{G}
$$

est de conoyau fini, et il en est de même du morphisme

$$
\operatorname{deg}_{T}: T\left(\boldsymbol{A}_{K}\right) \longrightarrow\left(X(T)^{G}\right)^{\vee} .
$$

Démonstration: C'est un cas particulier de [Oe, I.5.6.b], où la preuve est donnée pour tout groupe linéaire algébrique. Nous donnons ici une preuve pour les tores algébriques.

D'après le lemme 3.3 , il existe une suite exacte de $G$-modules

$$
0 \rightarrow X(T) \rightarrow P \rightarrow Q \rightarrow 0
$$

où $P$ et $Q$ sont libres de rang fini en tant que $\mathbf{Z}$-modules, et $P$ est un $G$ module de permutation. Notons $T_{P}$ (respectivement $T_{Q}$ ) le $K$-tore de module de caractère $P$ (respectivement $Q$ ).

Le conoyau de la flèche $\left(P^{\vee}\right)^{G} \rightarrow\left(X(T)^{\vee}\right)^{G}$ est le groupe $H^{1}\left(G, Q^{\vee}\right)$ qui est fini. Comme $T_{P}$ est quasi-déployé, d'après le lemme 3.15 le morphisme $\operatorname{deg}_{T_{P}, L}$ est de conoyau fini. Le diagramme commutatif

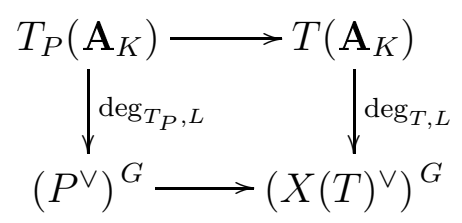

permet de conclure pour $\operatorname{deg}_{T, L}$. Or on a la formule

$$
d_{L} \operatorname{deg}_{T, L}=d_{K}[L: K] \operatorname{deg}_{T},
$$

et $\left(X(T)^{\vee}\right)^{G}$ est un sous-module d'indice fini de $\left(X(T)^{G}\right)^{\vee}$. On en déduit le résultat pour $\operatorname{deg}_{T}$.

\section{Notation 3.18}

Dans le cas fonctionnel, on note $\mathcal{C}_{T}$ le conoyau de $\operatorname{deg}_{T}$. 
On considère toujours $T$ un tore algébrique défini sur le corps de fonctions $K$. Les résultats de la fin de cette section donnent des renseignements sur les groupes $\mathcal{D}_{T}$ et $\mathcal{C}_{T}$. Soit $\mathcal{G}$ le groupe de Galois de $K^{\mathrm{s}} / K$, il contient un sous-groupe distingué $\mathcal{H}$ tel que le quotient $\mathcal{G} / \mathcal{H}$ s'identifie à $\mathfrak{G}$ le groupe de Galois absolu de $k$. La représentation continue de $\mathcal{G}$ dans $\operatorname{Aut}(X(T))$ induit une représentation continue

$$
\varrho: \mathfrak{G} \longrightarrow \operatorname{Aut}\left(X(T)^{\mathcal{H}}\right) .
$$

Soit $\mathfrak{g}_{T}=\mathfrak{G} / \operatorname{Ker}(\varrho)$ et $d_{T}=\left[\mathfrak{g}_{T}\right]$. Ainsi le corps fini à $q_{K}^{d_{T}}$ éléments est le corps des constantes minimal d'une extension galoisienne $L / K$ déployant $T$.

Pour tout $G$-module $M$, on note $N_{G}$ la norme sur $M$, i.e. le morphisme qui à $m \in M$ associe l'élément de $M^{G}$

$$
N_{G}(m)=\sum_{g \in G} g m
$$

\section{Lemme 3.19}

L'image de $N_{G} T\left(\boldsymbol{A}_{L}\right)$ par $\operatorname{deg}_{T}$ est $d_{T}\left(X(T)^{G}\right)^{\vee}$.

Démonstration : Soit

$$
\phi \in T\left(\mathbf{A}_{L}\right)=\operatorname{Hom}\left(X(T), \mathbf{G}_{m}\left(\mathbf{A}_{L}\right)\right) .
$$

Le morphisme $\operatorname{deg}_{T}\left(N_{G} \phi\right)$ envoie $m \in X(T)^{G}$ sur

$$
\begin{aligned}
\operatorname{deg}_{K}\left(\prod_{g \in G}(g \phi)(m)\right) & =\operatorname{deg}_{K}\left(\prod_{g \in G} g \cdot\left(\phi\left(g^{-1} m\right)\right)\right) \\
& =\operatorname{deg}_{K}\left(\prod_{g \in G} g \cdot(\phi(m))\right) \\
& =\frac{d_{T}}{[L: K]} \operatorname{deg}_{L}\left(\prod_{g \in G} g \cdot(\phi(m))\right) \\
& =d_{T} \operatorname{deg}_{L}(\phi(m)) .
\end{aligned}
$$

La troisième égalité provient de la formule (3.6), compte tenu du fait qu'on a $d_{T}=d_{L} / d_{K}$. Ainsi l'image de $N_{G} T\left(\mathbf{A}_{L}\right)$ par $\operatorname{deg}_{T}$ est incluse dans $d_{T}\left(X(T)^{G}\right)^{\vee}$.

Montrons à présent que la flèche

$$
\operatorname{deg}_{T}: N_{G} T\left(\mathbf{A}_{L}\right) \longrightarrow d_{T}\left(X(T)^{G}\right)^{\vee}
$$

est surjective. 
Soit $\psi$ un élément de $d_{T}\left(X(T)^{G}\right)^{\vee}$. On peut construire un morphisme

$$
\phi \in \operatorname{Hom}\left(X(T)^{G}, \mathbf{G}_{m}\left(\mathbf{A}_{L}\right)\right)
$$

tel que pour $m \in X(T)^{G}$ on ait

$$
\forall m \in X(T)^{G}, \quad \operatorname{deg}_{L}(\phi(m))=\frac{\psi(m)}{d_{T}} .
$$

Soit en effet $t \in \mathbf{G}_{m}\left(\mathbf{A}_{L}\right)$ un idèle tel que $\operatorname{deg}_{L}(t)=1$ Choisissons en outre une base de $X(T)^{G}\left(m_{1}, \ldots, m_{r}\right)$. Pour $i=1, \ldots, r, \psi\left(m_{i}\right)$ s'écrit $d_{T} n_{i}$ avec $n_{i} \in \mathbf{Z}$. On pose alors $\phi\left(m_{i}\right)=t^{n_{i}}$.

Comme $X(T)^{G}$ est en tant que $\mathbf{Z}$-module un facteur direct de $X(T)$, un tel morphisme $\phi$ s'étend en un morphisme

$$
\phi \in \operatorname{Hom}\left(X(T), \mathbf{G}_{m}\left(\mathbf{A}_{L}\right)\right)
$$

On vérifie que pour un tel $\phi$, on a, compte tenu de (3.89), $\operatorname{deg}_{T}\left(N_{G} \phi\right)=\psi$.

\section{Corollaire 3.20}

$\mathcal{D}_{T}$ contient $d_{T}\left(X(T)^{G}\right)^{\vee}$.

\section{Corollaire 3.21}

Si $k$ est algébriquement clos dans $L$, on a $\mathcal{C}_{T}=0$.

Démonstration : En effet dans ce cas on a $d_{T}=1$.

\subsection{Groupe de classes}

Rappelons que pour tout corps global $K, C_{K}$ désigne le groupe des classes d'idèles de $K$.

On considère un tore algébrique défini sur corps global $K$, déployé par une extension finie galoisienne $L$ de groupe $G$.

Le groupe

$$
T\left(C_{L}\right) \stackrel{\text { déf }}{=} \operatorname{Hom}\left(X(T), C_{L}\right)
$$

est isomorphe à $T\left(\mathbf{A}_{L}\right) / T(L)$. Il est par ailleurs muni naturellement d'une action de $G$. Nous posons

$$
T\left(C_{K}\right)=\operatorname{Hom}_{G}\left(X(T), C_{L}\right)=T\left(C_{L}\right)^{G} .
$$

Cette définition est indépendante du choix de l'extension déployant $T$. 
Notons que la suite exacte longue de cohomologie associée à la suite exacte de $G$-modules

$$
0 \longrightarrow X(T)^{\vee} \otimes \mathbf{G}_{m}(L) \longrightarrow X(T)^{\vee} \otimes \mathbf{G}_{m}\left(\mathbf{A}_{L}\right) \longrightarrow X(T)^{\vee} \otimes C_{L} \longrightarrow 0
$$

fournit la suite exacte

$$
0 \longrightarrow T(K) \longrightarrow T\left(\mathbf{A}_{K}\right) \longrightarrow T\left(C_{K}\right) \longrightarrow H^{1}(G, T(L)) \longrightarrow H^{1}\left(G, T\left(\mathbf{A}_{L}\right)\right)
$$

et donc la suite exacte

$$
0 \longrightarrow T\left(\mathbf{A}_{K}\right) / T(K) \longrightarrow T\left(C_{K}\right) \longrightarrow \amalg(T) \longrightarrow 0,
$$

où Ш(T) est le groupe de Tate-Shafarevich de $T$, défini par

$$
\amalg(T)=\operatorname{Ker}\left(H^{1}(G, T(L)) \rightarrow H^{1}\left(G, T\left(\mathbf{A}_{L}\right)\right)\right),
$$

cette définition ne dépendant pas du choix de l'extension déployant $T$.

Ainsi le groupe $T\left(\mathbf{A}_{K}\right) / T(K)$ s'injecte dans $T\left(C_{K}\right)$ mais ne lui est en général pas égal (pour un exemple avec $(T) \neq 0$ cf. par exemple [CTSa, p.224, §G.]).

Un cas important d'égalité se produit quand $T$ est la restriction à la Weil de $K_{0}$ à $K$ de $\mathbf{G}_{m}$, pour $K_{0}$ extension séparable de $K$. Dans ce cas, $T\left(C_{K}\right)$ s'identifie à $C_{K_{0}}$. En effet, on a alors, par le théorème de Hilbert 90,

$$
C_{K_{0}}=C_{L}^{\operatorname{Gal}\left(L / K_{0}\right)} .
$$

Bien entendu l'isomorphisme

$$
T\left(C_{K}\right) \stackrel{\sim}{\longrightarrow} T\left(\mathbf{A}_{K}\right) / T(K)
$$

est encore valable si $T$ est quasi-déployé.

\subsection{La dualité de Nakayama}

\subsubsection{Les groupes de cohomologie à la Tate}

Nous effectuons quelques rappels sur les propriétés des groupes de cohomologie à la Tate, renvoyant à [Se1, Chapitre VIII] pour plus de détails. Soit $G$ un groupe fini. Pour tout $n \in \mathbf{Z}$, on peut définir sur la catégorie des $G$-modules un foncteur $\widehat{H}^{n}(G$, . ), à valeurs dans la catégorie des $\mathbf{Z}$-modules, vérifiant entre autres les propriétés suivantes:

- Pour $n \geqslant 1$, ce foncteur coïncide avec le foncteur classique $H^{n}(G$, . ), $n$ ème foncteur dérivé droit du foncteur «points fixes sous $G »: M \mapsto M^{G}$. 
- Si $M$ est un $G$-module et

$$
N_{G}: m \mapsto \sum_{g \in G} g \cdot m
$$

est la norme, on a $\widehat{H}^{0}(G, M)=M^{G} / N_{G} M$.

$-\mathrm{Si}$

$$
0 \longrightarrow M^{\prime} \longrightarrow M \longrightarrow M^{\prime \prime} \longrightarrow 0
$$

est une suite exacte de $G$-modules, on a une suite exacte longue

$$
\begin{aligned}
& \cdots \longrightarrow \widehat{H}^{n-1}\left(G, M^{\prime \prime}\right) \stackrel{\delta}{\longrightarrow} \widehat{H}^{n}\left(G, M^{\prime}\right) \longrightarrow \widehat{H}^{n}(G, M) \\
& \longrightarrow \widehat{H}^{n}\left(G, M^{\prime \prime}\right) \stackrel{\delta}{\longrightarrow} \widehat{H}^{n+1}\left(G, M^{\prime}\right) \longrightarrow \widehat{H}^{n+1}(G, M) \ldots
\end{aligned}
$$

- Pour tout $n \in \mathbf{Z}, \widehat{H}^{n}(G, M)$ est tué par la multiplication par $[G]$.

- Si $M$ est de type fini, pour tout $n \in \mathbf{Z}, \widehat{H}^{n}(G, M)$ est fini.

- Si $M$ et $M^{\prime}$ sont des $G$-modules et $m$ et $n$ sont dans $\mathbf{Z}$, il existe une application Z-bilinéaire

$$
\widehat{H}^{m}(G, M) \otimes \widehat{H}^{n}\left(G, M^{\prime}\right) \longrightarrow \widehat{H}^{m+n}\left(G, M \otimes M^{\prime}\right) .
$$

fonctorielle en $M$ et $M^{\prime}$.

- Pour tout $G$-module $M$, le morphisme naturel de $G$-module

$$
M \otimes M^{\vee} \rightarrow \mathbf{Z}
$$

induit par fonctorialité un morphisme trace

$$
\widehat{H}^{0}\left(G, M \otimes M^{\vee}\right) \rightarrow \widehat{H}^{0}(G, \mathbf{Z}) \stackrel{\sim}{\rightarrow} \mathbf{Z} /[G] .
$$

En composant l'application bilinéaire (3.5) pour $M^{\prime}=M^{\vee}=\operatorname{Hom}(M, \mathbf{Z})$ et $n=-n$ avec la trace, on obtient une dualité parfaite

$$
\widehat{H}^{-n}(G, M) \otimes \widehat{H}^{n}\left(G, M^{\vee}\right) \longrightarrow \mathbf{Z} /[G]
$$

qui permet en particulier d'identifier $\widehat{H}^{-n}(G, M)$ au dual Hom $\left(\widehat{H}^{n}\left(G, M^{\vee}\right), \mathbf{Q} / \mathbf{Z}\right)$ de $\widehat{H}^{n}\left(G, M^{\vee}\right.$ (qui coïncide avec son dual topologique si $\widehat{H}^{n}\left(G, M^{\vee}\right)$ est fini), et inversement.

Dans toute la suite, pour tout $n \in \mathbf{Z}$, nous noterons $H^{n}(G, M)$ le groupe $\widehat{H}^{n}(G, M)$, sauf dans le cas où $n$ vaut explicitement 0 , où nous conserverons la notation $\widehat{H}^{0}(G, M)$ pour éviter toute confusion. 


\subsection{2 Énoncé de la dualité de Nakayama}

Soit $K$ un corps global et $T$ un tore algébrique sur $K$, déployé par une extension galoisienne $L$ de groupe $G$. La dualité de Nakayama donne un moyen simple de calculer la cohomologie du $G$-module $T\left(C_{L}\right)$. Le résultat suivant est une conséquence de [Na, Theorem 3]. La théorie du corps de classes permet de définir un générateur canonique $\alpha$ du groupe $H^{2}\left(G, C_{L}\right)$, appelé classe fondamentale (cf. [HoNa, p.359]).

\section{Théorème 3.22 (Nakayama)}

Le cup-produit par $\alpha$ induit pour tout $n \in Z$ un isomorphisme

$$
H^{n}\left(G, X(T)^{\vee}\right) \stackrel{\sim}{\longrightarrow} H^{n+2}\left(G, T\left(C_{L}\right)\right)
$$

En particulier les groupes de cohomologie $H^{n}\left(G, T\left(C_{L}\right)\right)$ sont finis pour tout $n$.

Rappelons que pour tout $n$ le groupe fini $H^{n}\left(G, X(T)^{\vee}\right)$ s'identifie canoniquement au dual $\operatorname{Hom}\left(H^{-n}(G, X(T)), \mathbf{Q} / \mathbf{Z}\right)=H^{-n}(G, X(T))^{*}$ du groupe fini $H^{-n}(G, X(T))$.

\subsection{Cocompacité}

Soit $K$ un corps global. Nous notons $C_{K}^{1}=\mathbf{G}_{m}\left(\mathbf{A}_{K}\right)^{1} / \mathbf{G}_{m}(K)$. C'est un groupe compact par [We1, IV§4, Theorem 6]. Soit à présent $T$ un tore algébrique sur $K$ déployé par une extension galoisienne finie $L$ de groupe $G$. Posons

$$
T\left(C_{K}\right)^{1}=\operatorname{Hom}_{G}\left(X(T), C_{L}^{1}\right),
$$

ce qui ne dépend pas du choix de l'extension $L$ déployant $K$.

\section{Lemme 3.23}

Le groupe $H^{1}\left(G, T\left(C_{L}\right)^{1}\right)$ est fini.

Démonstration : Dans le cas fonctionnel, on considère la suite exacte

$$
0 \longrightarrow X(T)^{\vee} \otimes C_{L}^{1} \longrightarrow X(T)^{\vee} \otimes C_{L} \stackrel{\operatorname{Id} \otimes \operatorname{deg}_{L}}{\longrightarrow} X(T)^{\vee} \longrightarrow 0 .
$$

La suite exacte de cohomologie sous $G$ associée fournit la suite exacte

$$
T\left(C_{K}\right) \longrightarrow\left(X(T)^{\vee}\right)^{G} \longrightarrow H^{1}\left(G, T\left(C_{L}^{1}\right)\right) \longrightarrow H^{1}\left(G, T\left(C_{L}\right)\right) .
$$

Par dualité de Nakayama, $H^{1}\left(G, T\left(C_{L}\right)\right)$ est fini et la flèche

$$
T\left(C_{K}\right) \longrightarrow\left(X(T)^{\vee}\right)^{G}
$$


n'est autre que la factorisation de $\operatorname{deg}_{T, L}$ par $T\left(C_{K}\right)$, son conoyau est donc fini.

Dans le cas arithmétique, on utilise la suite exacte

$$
0 \longrightarrow X(T)^{\vee} \otimes C_{L}^{1} \longrightarrow X(T)^{\vee} \otimes C_{L} \stackrel{\operatorname{Id} \otimes \operatorname{deg}_{L}}{\longrightarrow} X(T)_{\mathbf{R}}^{\vee} \longrightarrow 0
$$

et un argument strictement similaire (ici $\operatorname{deg}_{T, L}$ est d'ailleurs surjectif d'après le corollaire 3.14).

\section{Proposition 3.24}

1. Le quotient $T\left(\boldsymbol{A}_{K}\right)^{1} / T(K)$ est compact.

2. Dans le cas arithmétique, $T\left(\boldsymbol{A}_{K}\right) / \boldsymbol{K}(T) \cdot T(K) \cdot T\left(\boldsymbol{A}_{K}\right)_{\mathcal{P}_{K, \infty}}$ est fini.

3. Dans le cas fonctionnel, $T\left(\boldsymbol{A}_{K}\right)^{1} / \mathbf{K}(T) \cdot T(K)$ est fini.

Démonstration: Le point 1 est un cas particulier de [Oe, IV.1.3.], où il est montré plus généralement pour un groupe résoluble. Nous donnons une preuve de ce résultat pour un tore algébrique.

Si $T$ est quasi-déployé, le résultat découle immédiatemment de la compacité de $\mathbf{G}_{m}\left(\mathbf{A}_{L}\right)^{1} / \mathbf{G}_{m}(L)$ pour tout corps global $L$.

Par ailleurs, si $T$ est quasi-déployé. on a un isomorphisme

$$
T\left(C_{K}\right)^{1} \stackrel{\sim}{\longrightarrow} T\left(\mathbf{A}_{K}\right)^{1} / T(K)
$$

En effet, il suffit de le montrer pour $T=\operatorname{Res}_{K_{0} / K} \mathbf{G}_{m}$ où $K_{0}$ est une extension de $K$ contenue dans $L$. Or, par le théorème de Hilbert 90 , on a

$$
\left(C_{L}^{1}\right)^{\operatorname{Gal}\left(L / K_{0}\right)}=C_{K_{0}}^{1} .
$$

Ainsi, si $T$ est quasi-déployé, $T\left(C_{K}\right)^{1}$ est un compact.

Pour $T$ quelconque, d'après le lemme 3.3, il existe une suite exacte de $G$-modules

$$
0 \rightarrow X(T) \rightarrow P \rightarrow Q \rightarrow 0
$$

où $P$ et $Q$ libres de rang fini en tant que $\mathbf{Z}$-modules, et $P$ est un $G$-module de permutation. Notons $T_{P}$ (respectivement $T_{Q}$ ) le $K$-tore de module de caractère $P$ (respectivement $Q$ ).

On a le diagramme commutatif suivant

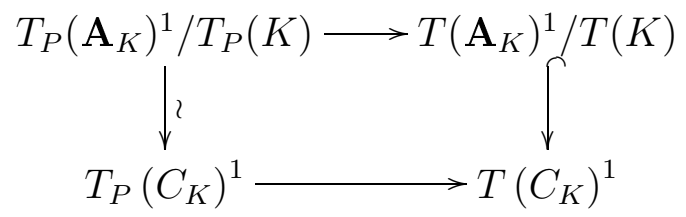


d'après lequel il suffit, pour montrer la compacité de $T\left(\mathbf{A}_{K}\right)^{1} / T(K)$, de montrer que la flèche $T_{P}\left(C_{K}\right)^{1} \rightarrow T\left(C_{K}\right)^{1}$ est de conoyau fini.

Or la suite exacte

$$
0 \longrightarrow Q^{\vee} \longrightarrow P^{\vee} \longrightarrow X(T)^{\vee} \longrightarrow 0
$$

fournit la suite exacte

$$
T_{P}\left(C_{K}\right)^{1} \longrightarrow T\left(C_{K}\right)^{1} \longrightarrow H^{1}\left(G, T_{Q}\left(C_{L}\right)^{1}\right) .
$$

D'après le lemme $3.23, H^{1}\left(G, T_{Q}\left(C_{L}\right)^{1}\right)$ est fini, d'où le résultat.

Montrons le point 2, qui, dans le cas où $T=\mathbf{G}_{m}$, est le théorème de finitude du nombre de classes d'idéaux d'un corps de nombres. D'après le lemme 3.13 , on a

$$
T\left(\mathbf{A}_{K}\right)=T\left(\mathbf{A}_{K}\right)^{1} \cdot T\left(\mathbf{A}_{K}\right)_{\mathcal{P}_{K, \infty}}
$$

et il suffit donc de montrer que le quotient

$$
T\left(\mathbf{A}_{K}\right)^{1} / \mathbf{K}(T) \cdot T(K) \cdot T\left(\mathbf{A}_{K}\right)_{\mathcal{P}_{K, \infty}}^{1}
$$

est fini. Mais ce dernier groupe est discret, et compact car $T\left(\mathbf{A}_{K}\right)^{1} / T(K)$ est compact d'après le point 1 .

Montrons à présent le point 3 . De fac̀ ;on semblable au point 2 , il peut se déduire du point 1 : la compacité de $T\left(\mathbf{A}_{K}\right)^{1} / T(K)$ entraîne celle de $T\left(\mathbf{A}_{K}\right)^{1} / \mathbf{K}(T) \cdot T(K)$, et donc la finitude de ce dernier groupe dans le cas fonctionnel car il est alors également discret.

Cependant le point 3 peut se retrouver directement, en remarquant qu'on a

$$
\left(T\left(\mathbf{A}_{L}\right)^{1}\right)^{G}=T\left(\mathbf{A}_{K}\right)^{1},
$$

d'où une injection

$$
T\left(\mathbf{A}_{K}\right)^{1} /\left(\mathbf{K}\left(T_{L}\right) \cdot T(L)\right)^{G} \hookrightarrow\left(T\left(\mathbf{A}_{L}\right)^{1} / \mathbf{K}\left(T_{L}\right) \cdot T(L)\right)^{G} .
$$

Or $T\left(\mathbf{A}_{L}\right)^{1} / \mathbf{K}\left(T_{L}\right) \cdot T(L)$ n'est autre que $\left(\operatorname{Pic}^{0}\left(\mathcal{C}_{L}\right)\right)^{\operatorname{dim}(T)}$. C'est donc un groupe fini. On en déduit que le groupe

$$
T\left(\mathbf{A}_{K}\right)^{1} /\left(\mathbf{K}\left(T_{L}\right) \cdot T(L)\right)^{G}
$$

est fini.

Par ailleurs, $\mathbf{K}\left(T_{L}\right) \cap T(L)$ s'identifie à un produit de $\operatorname{dim}(T)$ copies du groupe des fonctions régulières inversibles sur la courbe projective $\mathcal{C}_{L}$, et est donc fini. Il en en donc de même pour $H^{1}\left(G, \mathbf{K}\left(T_{L}\right) \cap T(L)\right)$. Maintenant la suite exacte

$$
0 \longrightarrow \mathbf{K}(T) \cdot T(K) \longrightarrow\left(\mathbf{K}\left(T_{L}\right) \cdot T(L)\right)^{G} \longrightarrow H^{1}\left(G, \mathbf{K}\left(T_{L}\right) \cap T(L)\right)
$$

montre que $\mathbf{K}(T) \cdot T(K)$ est d'indice fini dans $\left(\mathbf{K}\left(T_{L}\right) \cdot T(L)\right)^{G}$, d'où la finitude de $T\left(\mathbf{A}_{K}\right)^{1} / \mathbf{K}(T) \cdot T(K)$. 


\subsection{Résultats locaux}

\section{Lemme 3.25 (Ono)}

Soit $v$ une place finie de $K$. Soit $1 \rightarrow T^{\prime \prime} \rightarrow T^{\prime} \rightarrow T \rightarrow 1$ une suite exacte de tores algébriques sur $K$. Soit $L$ une extension galoisienne finie déployant $T$, $T^{\prime}$ et $T$ ". Si $v$ est non ramifiée dans $L / K$, le morphisme

$$
T^{\prime}\left(\mathcal{O}_{v}\right) \longrightarrow T\left(\mathcal{O}_{v}\right)
$$

est surjectif.

Démonstration : La preuve figure dans [On1, Lemma 4.2.1], nous la rappelons. Soit $\mathcal{V}$ une place de $L$ au-dessus de $v$, et $G_{v}$ le groupe de décomposition de $\mathcal{V}$. On a

$$
\begin{aligned}
& T\left(\mathcal{O}_{v}\right) \stackrel{\sim}{\rightarrow} \\
& T^{\prime} \operatorname{Hom}_{G_{v}}\left(X(T), \mathcal{O}_{\mathcal{V}}^{\times}\right), \\
& T^{\prime}\left(\mathcal{O}_{v}\right) \stackrel{\sim}{\rightarrow} \operatorname{Hom}_{G_{v}}\left(X\left(T^{\prime}\right), \mathcal{O}_{v}^{\times}\right)
\end{aligned}
$$

et une suite exacte

$$
T^{\prime}\left(\mathcal{O}_{v}\right) \longrightarrow T\left(\mathcal{O}_{v}\right) \longrightarrow H^{1}\left(G_{v}, X\left(T^{\prime \prime}\right)^{\vee} \otimes \mathcal{O}_{v}^{\times}\right) .
$$

Or, comme $v$ est non ramifiée, $\mathcal{O}_{V}^{\times}$est cohomologiquement trivial, et comme $X\left(T^{\prime \prime}\right)^{\vee}$ est sans torsion, d'après [Se1, IX, § 5, Corollaire], $X\left(T^{\prime \prime}\right)^{\vee} \otimes$ $\mathcal{O}_{v}^{\times}$est encore cohomologiquement trivial, d'où le résultat.

Soit $T$ un tore algébrique défini sur un corps global $K$, déployé par une extension finie galoisienne $L$ de groupe de Galois $G$. On suppose donnée une suite exacte de $G$-modules

$$
0 \longrightarrow X(T) \stackrel{\gamma}{\longrightarrow} P \longrightarrow Q \longrightarrow 0
$$

où $P$ et $Q$ sont des $\mathbf{Z}$-modules libres de rang fini, et $P$ est un $G$-module de permutation. Le but de ce qui suit est de préciser le comportement du degré local vis-à-vis de cette suite exacte. Comme conséquence, on obtient la finitude du conoyau du degré local.

Choisissons une base $G$-stable $\left(n_{i}\right)_{i \in I}$ du $G$-module de permutation $P$, dont on note $\left(n_{i}^{\vee}\right)_{i \in I}$ la base duale. Pour $\tilde{\imath} \in I / G$, choisissons en outre un élément $n_{\tilde{\imath}}$ de l'orbite $\widetilde{\imath}$. Soit $G_{\tilde{\imath}}$ son stabilisateur. Ces choix permettent d'identifier $P$ (et $P^{\vee}$ ) au $G$-module de permutation $\underset{\tilde{\imath} \in I / G}{\oplus} \mathbf{Z}\left[G / G_{\bar{\imath}}\right]$. Si on désigne par $K_{\tilde{\imath}}$ le corps $L^{G_{\tilde{\imath}}}$, cette identification iduit un isomorphisme

$$
T_{P} \stackrel{\sim}{\longrightarrow} \prod_{\tilde{\imath} \in I / G} \operatorname{Res}_{K_{\tilde{\imath}}, K} \mathbf{G}_{m} .
$$


Soit $v$ une place finie de $K, \mathcal{V}$ une place de $L$ divisant $v$ et $G_{v}$ son groupe de décomposition. Rappelons qu'on note encore $\mathcal{V}$ la valuation normalisée représentant $\mathcal{V}$.

L'isomorphisme (3.6) induit au niveau des $K_{v}$-points un isomorphisme

$$
T_{P}\left(K_{v}\right) \stackrel{\sim}{\longrightarrow} \prod_{\tilde{\imath} \in I / G} \mathbf{G}_{m}\left(K_{\tilde{\imath}} \otimes K_{v}\right)=\prod_{\tilde{\imath} \in I / G} \prod_{\substack{w \in \mathcal{P}_{K_{\tilde{\imath}}} \\ w \mid v}} \mathbf{G}_{m}\left(K_{w}\right) .
$$

Pour $\widetilde{\imath} \in I / G$, considérons l'application

$$
\begin{aligned}
G / G_{\widetilde{\imath}} & \longrightarrow\left\{w \in \mathcal{P}_{K_{\tilde{\imath}}}, v \mid w\right\} \\
g & \left.\longmapsto g^{-1} \cdot \mathcal{V}\right|_{K_{\tilde{\imath}}}
\end{aligned}
$$

Ce n'est autre que le passage au quotient par l'action de $G_{v}$, d'où une correspondance entre $\widetilde{\imath} / G_{v}$ et les places de $K_{\widetilde{\imath}}$ au-dessus de $v$.

Pour $j \in \tilde{\imath} / G_{v}$, nous notons $w_{j}$ la place de $K_{\widetilde{\imath}}$ au-dessus de $v$ donnée par cette correspondance. On choisit en outre $n_{j}$ un élément quelconque de $j$.

On pose alors

$$
\tau_{j}=\sum_{n \in G_{v} \cdot n_{j}} \gamma^{\vee}\left(n^{\vee}\right)
$$

de sorte que $\tau_{j}$ est un élément de $\left(X(T)^{\vee}\right)^{G_{v}}$.

$\operatorname{Pour} \tilde{\imath} \in I / G$ et $j \in \widetilde{\imath} / G_{v}$, on identifie, via l'isomorphisme (3.7), $\mathbf{G}_{m}\left(K_{w_{j}}\right)$ à un sous-groupe de $T_{P}\left(K_{v}\right)$. Soit $\pi_{w_{j}}$ une uniformisante de $K_{w_{j}}$ et $e_{j}$ l'indice de ramification de $w_{j}$ dans $L$.

\section{Lemme 3.26}

On a la relation

$$
\operatorname{deg}_{T, L, \mathcal{V}}\left[\gamma_{v}\left(\pi_{w_{j}}\right)\right]=e_{j} \tau_{j}
$$

Démonstration : Le morphisme $\gamma$ et la décomposition

$$
P \stackrel{\sim}{\rightarrow} \bigoplus_{\tilde{\imath} \in I / G} \bigoplus_{j \in \widetilde{\imath} / G_{v}} \underset{n \in G_{v} . n_{j}}{\oplus} \mathbf{Z} n
$$

induisent des morphismes de $G_{v}$-module

$$
X(T) \stackrel{\gamma_{j}}{\longrightarrow} \bigoplus_{n \in G_{v} . n_{j}} \mathbf{Z} n
$$

qui à leur tour induisent par dualité des morphismes de $K_{v}$-tores

$$
\operatorname{Res}_{K_{w_{j}} / K_{v}} \mathbf{G}_{m} \longrightarrow T
$$


Pour tout $j$, ce dernier morphisme induit au niveau des $K_{v}$-points un morphisme

$$
\operatorname{Res}_{K_{w_{j}} / K_{v}} \mathbf{G}_{m}\left(K_{v}\right) \stackrel{\sim}{\rightarrow} \mathbf{G}_{m}\left(K_{w_{j}}\right) \longrightarrow T\left(K_{v}\right)
$$

qui n'est autre que la restriction de $\gamma_{v}$ à $\mathbf{G}_{m}\left(K_{w_{j}}\right)$.

Il peut donc se décrire comme le morphisme

$$
\operatorname{Hom}_{G_{v}}\left(\underset{n \in G_{v} . n_{j}}{\oplus} \mathbf{Z} n, L_{\mathcal{V}}^{\times}\right) \stackrel{\circ \gamma_{j}}{\longrightarrow} \operatorname{Hom}_{G_{v}}\left(X(T), L_{\mathcal{V}}^{\times}\right) .
$$

On calcule alors $\gamma_{v}\left(\pi_{w_{j}}\right)$ en utilisant la description (3.15).

Via l'identification

$$
\mathbf{G}_{m}\left(K_{w_{j}}\right) \stackrel{\sim}{\rightarrow} \operatorname{Hom}_{G_{v}}\left(\underset{n \in G_{v} . n_{j}}{\oplus} \mathbf{Z} n, L_{\mathcal{V}}\right)
$$

l'élément $\pi_{w_{j}}$ de $\mathbf{G}_{m}\left(K_{w_{j}}\right)$ correspond au morphisme qui envoie $n_{j}$ sur $\pi_{w_{j}}$

Son image par $\gamma_{v}$ est donc l'élément de $\operatorname{Hom}_{G_{v}}\left(X(T), L_{\mathcal{V}}^{\times}\right)$donné par

$$
m \mapsto \prod_{n \in G_{v}, n_{j}}\left(g_{n} \pi_{w_{j}}\right)^{\left\langle m, \gamma^{\vee}\left(n^{\vee}\right)\right\rangle}
$$

où pour $n \in G_{v} . n_{j}$ on note $g_{n}$ un élément de $G_{v}$ tel que $n=g_{n} \cdot n_{j}$.

On en déduit que $\operatorname{deg}_{T, L, v}\left(\gamma_{v}\left(\pi_{w_{j}}\right)\right)$ est l'élément de $\left(X(T)^{\vee}\right)^{G_{v}}$ qui à $m \in X(T)$ associe

$$
\begin{aligned}
\sum_{n \in G_{v} . n_{j}}\left\langle m, \gamma^{\vee}\left(n^{\vee}\right)\right\rangle \mathcal{V}\left(g_{n} \pi_{w_{j}}\right) & =\sum_{n \in G_{v} . n_{j}}\left\langle m, \gamma^{\vee}\left(n^{\vee}\right)\right\rangle \mathcal{V}\left(\pi_{w_{j}}\right) \\
& =\left\langle m, e_{j} \sum_{n \in G_{v} . n_{j}} \gamma^{\vee}\left(n^{\vee}\right)\right\rangle \\
& =\left\langle m, e_{j} \tau_{j}\right\rangle
\end{aligned}
$$

d'où le résultat.

Comme conséquence du lemme 3.26, on obtient le résultat suivant, qui est signalé à la page 449 de [Dr].

\section{Proposition 3.27}

Le morphisme

$$
\operatorname{deg}_{T, L, \mathcal{V}}: T\left(K_{v}\right) \longrightarrow\left(X(T)^{\vee}\right)^{G_{v}}
$$

est de conoyau fini. 
Remarque 3.28: Si en outre $v$ n'est pas ramifiée dans $L$, on a déjà vu que le conoyau est en fait trivial (cf. le lemme 3.9).

Démonstration : Comme $H^{1}\left(G_{v}, Q^{\vee}\right)$ est fini, la flèche $\left(P^{\vee}\right)^{G_{v}} \rightarrow\left(X(T)^{\vee}\right)^{G_{v}}$ (induite par la restriction de $\left.\gamma^{\vee} \grave{a}\left(P^{\vee}\right)^{G_{v}}\right)$ est de conoyau fini. Comme $\left(P^{\vee}\right)^{G_{v}}$ est engendré par les éléments

$$
\sum_{n \in G_{v} \cdot n_{j}} n^{\vee}
$$

pour $\widetilde{\imath}$ décrivant $I / G$ et $j$ décrivant $\widetilde{\imath} / G_{v}$, on voit que le sous-Z-module de $\left(X(T)^{\vee}\right)^{G_{v}}$ engendré par les $\tau_{j}$ pour $j \in \widetilde{\imath} / G_{v}$ et $\tilde{\imath} \in I / G$ est d'indice fini dans $\left(X(T)^{\vee}\right)^{G_{v}}$. Il en est donc de même du sous-Z-module engendré par les $e_{j} \tau_{j}$. Or, le lemme 3.26 montre que $\operatorname{deg}_{T, L, v}\left(\gamma_{v}\left(T_{P}\left(K_{v}\right)\right)\right)$ contient ce dernier sous-module.

Remarque 3.29: Le raisonnement précédent pourrait permettre en fait de montrer en une seule fois la proposition 3.27 et le lemme 3.9, en choisissant la suite exacte (3.5) de sorte que $Q$ soit un $G$-module flasque (cf. la sous-section 3.8.1). En effet, dans ce cas, la flèche $\left(P^{\vee}\right)^{G_{v}} \rightarrow\left(X(T)^{\vee}\right)^{G_{v}}$ est surjective, et non plus seulement de conoyau fini. Or, si $v$ n'est pas ramifiée dans $L$, tous les $e_{j}$ sont égaux à 1 , et le raisonnement précédent montre que $\operatorname{deg}_{T, L, \nu}$ est surjective.

\subsection{Résolution flasque d'un tore algébrique et applica- tions}

\subsubsection{Rappels et notations}

La notion de résolution flasque d'un tore algébrique a été introduite par Colliot-Thélène et Sansuc dans [CTSa] en vue de l'étude de la R-équivalence sur les tores.

Soit $T$ un tore algébrique défini sur un corps $K$, déployé par une extension finie galoisienne $L$ de groupe de Galois $G$. Rappelons qu'un $G$-module $M$ est dit flasque si pour tout sous-groupe $H$ de $G$ on a $H^{-1}(H, M)=0$. Par [CTSa, lemme 3, page 181] il existe une suite exacte de $G$-modules

$$
0 \longrightarrow X(T) \stackrel{\gamma}{\longrightarrow} P \longrightarrow Q \longrightarrow 0
$$

où $P$ et $Q$ sont libres de rang fini comme $\mathbf{Z}$-modules, $P$ est de permutation et $Q$ est flasque (ceci généralise le lemme 3.3). Un telle suite exacte est appelée une résolution flasque de $X(T)$. 
On fixe pour toute la section 3.8 un tore algébrique $T$ défini sur un corps global $K$, déployé par une extension finie galoisienne $L$ de groupe de Galois $G$. et une résolution flasque (3.1) de $X(T)$. Soit $T_{P}$ le tore algébrique associé au $G$-module $P$. C'est un tore quasi-déployé. Soit $T_{Q}$ le tore algébrique associé au $G$-module $Q$.

\subsubsection{Un résultat local}

On conserve les hypothèses et notations introduites dans la sous-section 3.8.1.

On considère dans cette partie une place finie $v$ de $K$. On note $\mathcal{V}$ une place de $L$ au-dessus de $v$ et $G_{v}$ le groupe de décomposition de $\mathcal{V}$.

\section{Proposition 3.30}

Si $v$ est non ramifiée dans $L / K$, le morphisme

$$
\gamma_{v}: T_{P}\left(K_{v}\right) \longrightarrow T\left(K_{v}\right)
$$

est surjectif.

Démonstration : La suite exacte de cohomologie associée à la suite exacte de $G_{v}$-modules

$$
1 \rightarrow T_{Q}\left(L_{\mathcal{V}}\right) \rightarrow T_{P}\left(L_{\mathcal{V}}\right) \rightarrow T\left(L_{\mathcal{V}}\right) \rightarrow 1
$$

et le fait que $T_{P}$ soit déployé, donc que $H^{1}\left(G_{v}, T_{P}\left(L_{\mathcal{V}}\right)\right)$ soit nul d'après Hilbert 90, montre que le conoyau de $\gamma_{v}$ est

$$
H^{1}\left(G_{v}, T_{Q}\left(L_{\mathcal{V}}\right)\right)=H^{1}\left(G_{v}, Q^{\vee} \otimes L_{\mathcal{V}}^{\times}\right) .
$$

La nullité de ce groupe découle de l'application de la dualité de Nakayama aux corps locaux : celle-ci fournit un isomorphisme

$$
H^{1}\left(G_{v}, Q^{\vee} \otimes L_{\mathcal{V}}^{\times}\right) \stackrel{\sim}{\longrightarrow} H^{-1}\left(G_{v}, Q^{\vee}\right) .
$$

Par ailleurs, $v$ étant non ramifiée, $G_{v}$ est cyclique, d'où un isomorphisme

$$
H^{-1}\left(G_{v}, Q^{\vee}\right) \stackrel{\sim}{\longrightarrow} H^{1}\left(G_{v}, Q^{\vee}\right) .
$$

Or le groupe $H^{1}\left(G_{v}, Q^{\vee}\right)$ est nul car $Q$ est flasque.

\section{Corollaire 3.31}

On se place dans le cas fonctionnel et on suppose que le tore $T$ est déployé par une extension $L / K$ non ramifiée. Alors le morphisme $T_{P}\left(\boldsymbol{A}_{K}\right) \rightarrow T\left(\boldsymbol{A}_{K}\right)$ est surjectif.

Démonstration: Ceci découle de la proposition 3.30 et du lemme 3.25. 


\subsubsection{Approximation faible}

On conserve les hypothèses et notations introduites dans la sous-section 3.8.1.

Soit $\overline{T(K)}$ l'adhérence de $T(K)$ dans $\prod_{v \in \mathcal{P}_{K}} T\left(K_{v}\right)$ muni de la topologie produit et soit

$$
A(T)=\left(\prod_{v \in \mathcal{P}_{K}} T\left(K_{v}\right)\right) / \overline{T(K)},
$$

c'est le groupe d'obstruction à l'approximation faible. Il est nul si $T=\mathbf{G}_{m}$ et plus généralement si $T$ est quasi-déployé, d'après [Ha, p. 334].

Soit $p$ le morphisme $T\left(\mathbf{A}_{K}\right) / T(K) \rightarrow A(T)$ induit par la projection $T\left(\mathbf{A}_{K}\right) \rightarrow A(T)$.

Pour tout ensemble fini $S$ de places de $K$ contenant les places archimédiennes, nous notons

$$
T\left(\mathbf{A}_{K}\right)^{S}=T\left(\mathbf{A}_{K}\right) \bigcap \prod_{v \notin S} T\left(K_{v}\right) .
$$

et

$$
\overline{T(K)}{ }^{S}=\overline{T(K)} \bigcap \prod_{v \in S} T\left(K_{v}\right)
$$

(en d'autres termes, $\overline{T(K)}$ S est l'adhérence de l'image de $T(K)$ dans $\prod_{v \in S} T\left(K_{v}\right)$ ).

\section{Proposition 3.32}

1. On suppose que $S$ contient les places ramifiées dans $L / K$. On a alors un scindage

$$
\overline{T(K)}=\left(\prod_{v \notin S} T\left(K_{v}\right)\right) \times \overline{T(K)} S
$$

En particulier on a un scindage

$$
\overline{T(K)} \cap T\left(\boldsymbol{A}_{K}\right)=T\left(\boldsymbol{A}_{K}\right)^{S} \times \overline{T(K)} S
$$

et les égalités

$$
A(T)=\left(\prod_{v \in S} T\left(K_{v}\right)\right) \overline{T(K)}^{S}=T\left(\boldsymbol{A}_{K}\right) /\left(\overline{T(K)} \cap T\left(\boldsymbol{A}_{K}\right)\right) .
$$

2. La suite

$$
T_{P}\left(\boldsymbol{A}_{K}\right) / T_{P}(K) \longrightarrow T\left(\boldsymbol{A}_{K}\right) / T(K) \stackrel{p}{\longrightarrow} A(T) \longrightarrow 0
$$

est exacte. 
3. Il existe une suite exacte

$$
0 \longrightarrow A(T) \longrightarrow H^{1}(G, Q)^{*} \longrightarrow \amalg(T) \longrightarrow 0 .
$$

En particulier, $A(T)$ est fini et on a

$$
[A(T)]=\frac{\left[H^{1}(G, Q)\right]}{[\amalg(T)]} .
$$

Remarques 3.33 :

1. Le point 1 est dû à Voskresenskii.

2. Dans le cas des corps de nombres, le point 2 est indiqué dans [BaTs1, Theorem 3.1.1].

3. Le point 3 a été initialement démontré par Voskresenskii en caractéristique zéro et sous une forme légèrement différente ([Vo, Thm. 6]). Il est dû sous la forme donnée ici à Colliot-Thélène et Sansuc ([CTSa, Proposition 19 (iB)]). La démonstration qui en est faite ci-dessous reprend, à des détails de présentation près, celle des auteurs de [CTSa]. Elle figure dans ce texte d'une part par souci de complétude, d'autre part parce que les arguments utilisés permettent aussi d'obtenir le point 2 .

4. Si on ne suppose plus $Q$ flasque dans la suite exacte 3.1, Draxl montre dans $[\mathrm{Dr}]$ que le conoyau du morphisme

$$
T_{P}\left(\mathbf{A}_{K}\right) / T_{P}(K) \longrightarrow T\left(\mathbf{A}_{K}\right) / T(K)
$$

est de cardinal

$$
\frac{\left[H^{1}(G, Q)\right]}{\amalg(T)} .
$$

Ce résultat permet de retrouver le point 2 à partir du point 3 .

Il permet aussi de montrer que les points 2 et 3 ne sont pas nécessairement vérifiés si on ne suppose plus $Q$ flasque dans la suite exacte 3.1 (contrairement à ce qui est affirmé en haut de la page 3231 de [BaTs4]). Considérons par exemple le cas où $G$ est cyclique, et où la suite exacte

$$
0 \longrightarrow X(T) \longrightarrow P \longrightarrow Q \longrightarrow 0
$$

est la suite duale de la suite exacte

$$
0 \longrightarrow I_{G} \longrightarrow \mathbf{Z}[G] \stackrel{\varepsilon}{\longrightarrow} \mathbf{Z} \longrightarrow 0
$$

où $\varepsilon$ est l'augmentation $\sum a_{g} g \mapsto \sum a_{g}$.

Comme $T$ est déployé, on a $A(T)=1$, et d'après le résultat de Draxl le conoyau de la flèche $T_{P}\left(\mathbf{A}_{K}\right) / T_{P}(K) \rightarrow T\left(\mathbf{A}_{K}\right) / T(K)$ est de cardinal $[G]$. 
5. La relation (3.15) nous servira pour le calcul du terme principal de la fonction zêta des hauteurs dans la section 8 .

\section{Démonstration :}

Comme $T_{P}$ est quasi-déployé, on a $A\left(T_{P}\right)=0$ soit en d'autres termes $\prod_{v} T_{P}\left(K_{v}\right)=\overline{T_{P}(K)}$. Ceci ajouté au fait que $\prod_{v} \gamma_{v}$ est continu pour la topologie produit montre les inclusions

$$
\left(\prod_{v} \gamma_{v}\right)\left(\prod_{v} T_{P}\left(K_{v}\right)\right) \subset \overline{\left(\prod_{v} \gamma_{v}\right)\left(T_{P}(K)\right)} \subset \overline{T(K)} .
$$

Or, d'après la proposition 3.30, l'image de $\prod_{v \notin S} T_{P}\left(K_{v}\right)$ par $\prod_{v} \gamma_{v}$ est $\prod_{v \notin S} T\left(K_{v}\right)$. On en déduit que $\overline{T(K)}$ contient $\prod_{v \notin S} T\left(K_{v}\right)$, ce qui, compte tenu du fait que $\overline{T(K)}$ est un sous-groupe de $\prod_{v} T\left(K_{v}\right)$, montre l'égalité (3.10). Les égalités (3.11) et (3.12) en découle aussitôt.

Pour la suite de la preuve, on considère le diagramme commutatif et exact

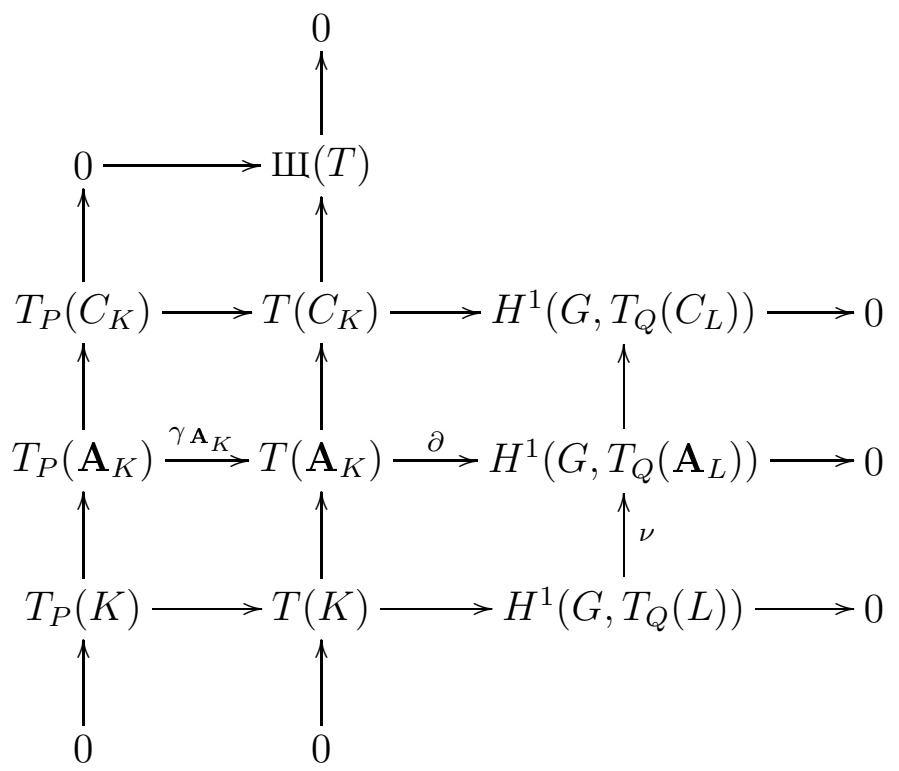

La deuxième (respectivement troisième, respectivement quatrième) ligne s'obtient à partir de la suite exacte longue de cohomologie tirée de la suite obtenue en tensorisant le dual de la suite exacte (3.1) avec $C_{L}$ (respectivement $\mathbf{G}_{m}\left(\mathbf{A}_{L}\right)$, respectivement $\left.\mathbf{G}_{m}(L)\right)$, en prenant les $G$-invariants, et en remarquant que : 
- par dualité de Nakayama,

$$
H^{1}\left(G, P^{\vee} \otimes C_{L}\right) \stackrel{\sim}{\longrightarrow} H^{1}(G, P)^{*}
$$

et $P$ étant de permutation, $H^{1}(G, P)=0$;

- $H^{1}\left(G, T_{P}\left(\mathbf{A}_{L}\right)\right)$ et $H^{1}\left(G, T_{P}(L)\right)$ sont nuls; ceci découle du fait que $T_{P}$ est quasi-déployé et de Hilbert 90 (si $k^{\prime} / k$ est une extension finie galoisienne de corps, $H^{1}\left(\mathrm{Gal}\left(k^{\prime} / k\right), \mathbf{G}_{m}(k)\right)=0$; si en outre $k$ est global, $\left.H^{1}\left(\operatorname{Gal}\left(k^{\prime} / k\right), \mathbf{G}_{m}\left(\mathbf{A}_{k^{\prime}}\right)\right)=0\right)$.

La première (respectivement deuxième, respectivement troisième) colonne s'obtient à partir de la suite exacte longue de cohomologie tirée de la suite obtenue en tensorisant la suite exacte

$$
1 \longrightarrow \mathbf{G}_{m}(L) \longrightarrow \mathbf{G}_{m}\left(\mathbf{A}_{L}\right) \longrightarrow C_{L}
$$

par $P^{\vee}$ (respectivement $\left.X(T)^{\vee}\right)$, respectivement $Q^{\vee}$ ), de la définition de $\amalg(T)$ et du fait que $T_{P}$ étant quasi-déployé, on a $\amalg\left(T_{P}\right)=0$.

Nous allons montrer que le sous-groupe $\partial^{-1}\left(\nu\left(H^{1}\left(G, T_{Q}(L)\right)\right.\right.$ est égal à $\overline{T(K)} \cap T\left(\mathbf{A}_{K}\right)$, soit, en d'autre termes, que le groupe $A(T)$ s'identifie au conoyau de $\nu$.

De (3.20) on déduit aussitôt l'inclusion

$$
\operatorname{Ker}(\partial)=\gamma_{\mathbf{A}_{K}}\left(T_{P}\left(\mathbf{A}_{K}\right)\right) \subset \overline{T(K)} \cap T\left(\mathbf{A}_{K}\right)
$$

Du diagramme (3.21) on déduit l'égalité

$$
\partial^{-1}\left(\nu \left(H^{1}\left(G, T_{Q}(L)\right)=T(K) \cdot \operatorname{Ker}(\partial)\right.\right.
$$

soit

$$
\partial^{-1}\left(\nu \left(H^{1}\left(G, T_{Q}(L)\right) \subset \overline{T(K)} \cap T\left(\mathbf{A}_{K}\right)\right.\right.
$$

Par ailleurs on a vu que l'image de $\prod_{v} T_{P}\left(K_{v}\right)$ par $\prod_{v} \gamma_{v}$ était égale à

$$
\prod_{v \notin S} T\left(K_{v}\right) \times \prod_{v \in S} \gamma_{v}\left(T_{P}\left(K_{v}\right)\right)
$$

Or, pour tout $v, \gamma_{v}$ est ouverte d'après [Sa, Proposition 2.7(a)].

Ainsi l'image de $\prod_{v} T_{P}\left(K_{v}\right)$ par $\prod_{v} \gamma_{v}$ est ouverte dans $\prod_{v} T\left(K_{v}\right)$.

Mais, d'après le lemme 3.25, on a

$$
\gamma_{\mathbf{A}_{K}}\left(T_{P}\left(\mathbf{A}_{K}\right)\right)=T\left(\mathbf{A}_{K}\right) \bigcap\left(\prod_{v} \gamma_{v}\right)\left(\prod_{v} T_{P}\left(K_{v}\right)\right) .
$$


En particulier, l'image de $T_{P}\left(\mathbf{A}_{K}\right)$ par $\gamma_{\mathbf{A}_{K}}$ est ouverte dans $T\left(\mathbf{A}_{K}\right)$ pour la topologie produit. Or cette image n'est autre que $\operatorname{Ker}(\partial)$. Ainsi le sousgroupe $\partial^{-1}\left(\nu\left(H^{1}\left(G, T_{Q}(L)\right)\right.\right.$ est ouvert dans $T\left(\mathbf{A}_{K}\right)$ (toujours pour la topologie produit), donc fermé. Comme il contient $T(K)$, on a

$$
\overline{T(K)} \cap T\left(\mathbf{A}_{K}\right) \subset \partial^{-1}\left(\nu \left(H^{1}\left(G, T_{Q}(L)\right)\right.\right.
$$

De (3.26) et (3.29) on déduit l'égalité cherchée

$$
\partial^{-1}\left(\nu \left(H^{1}\left(G, T_{Q}(L)\right)=\overline{T(K)} \cap T\left(\mathbf{A}_{K}\right)\right.\right.
$$

Le fait que le groupe $A(T)$ s'identifie au conoyau de $\nu$ a deux conséquences : d'une part le lemme du serpent et le diagramme commutatif exact

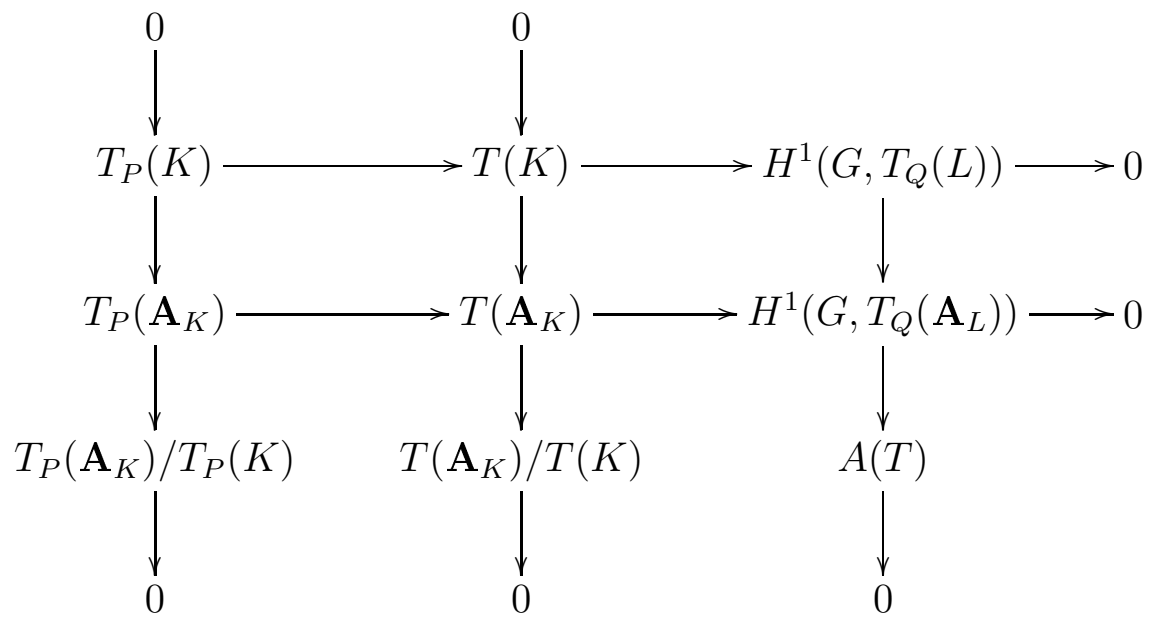

montrent le point 3 .

D'autre part, une chasse au diagramme standard dans (3.21) montre l'existence d'une suite exacte

$$
0 \longrightarrow A(T) \longrightarrow H^{1}\left(G, T_{Q}\left(C_{L}\right)\right) \longrightarrow \amalg(T) \longrightarrow 0 .
$$

Par dualité de Nakayama, on a un isomorphisme

$$
H^{1}\left(G, T_{Q}\left(C_{L}\right)\right) \stackrel{\sim}{\longrightarrow} H^{1}(G, Q)^{*}
$$

d'où la suite exacte (3.14).

\section{Corollaire 3.34}

On se place dans le cas fonctionnel et on suppose que le tore $T$ est déployé par une extension $L / K$ non ramifiée. Alors $A(T)$ est trivial.

Démonstration : Ceci découle du corollaire 3.31 et du point 2 de la proposition 3.32 . 


\subsubsection{Un invariant des tores algébriques définis sur les corps de fonctions}

On introduit dans cette sous-section un invariant des tores algébriques définis un corps de fonctions. Cet invariant intervient naturellement dans le calcul du terme principal de la fonction zêta des hauteurs d'une variétés torique en caractéristique positive. La question de savoir si cet invariant est trivial ou non n'est nullement évidente, et a été résolue par Colliot-Thélène et Suresh. Comme déjà indiqué, dans une version précédente de ce texte, il était affirmé à tort que cet invariant subsistait dans l'expression finale du terme principal de la fonction zêta des hauteurs des variétés toriques.

On conserve les hypothèse et notations introduites dans la sous-section 3.8.1. et on suppose en outre dans cette sous-section que $K$ est un corps de fonctions, de corps des constantes $k$. De la résolution flasque (3.1) on tire au niveau des espaces adéliques la suite exacte

$$
0 \longrightarrow T_{Q}\left(\mathbf{A}_{K}\right) \longrightarrow T_{P}\left(\mathbf{A}_{K}\right) \longrightarrow T\left(\mathbf{A}_{K}\right) .
$$

Enfin, en prenant l'image de cette suite exacte par l'application $\operatorname{deg}_{T}$ (cf. section 3.3), on obtient un complexe

$$
\mathcal{D}_{T_{Q}} \longrightarrow \mathcal{D}_{T_{P}} \longrightarrow \mathcal{D}_{T}
$$

\section{Lemme 3.35}

Le conoyau de la flèche $\mathcal{D}_{T_{P}} \longrightarrow \mathcal{D}_{T}$ est fini, et il ne dépend pas du choix de la résolution flasque.

Démonstration : On a le diagramme commutatif suivant

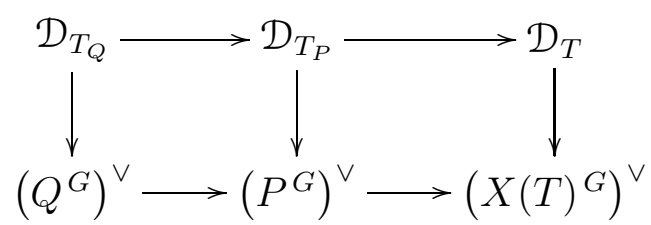

où les flèches verticales sont de conoyau fini. Par ailleurs le quotient $P^{G} / X(T)^{G}$ s'injecte dans $Q^{G}$, et est donc sans torsion. Ainsi le morphisme $\left(P^{G}\right)^{\vee} \rightarrow$ $\left(X(T)^{G}\right)^{\vee}$ est surjectif, et donc la flèche

$$
\mathcal{D}_{T_{P}} \longrightarrow \mathcal{D}_{T}
$$

est de conoyau fini.

Montrons que ce conoyau ne dépend pas du choix de la résolution flasque. En effet soit $P_{1}$ un $G$-module de permutation, on obtient à partir de (3.1) une nouvelle résolution flasque

$$
\mathcal{R}_{1}: 0 \longrightarrow X(T) \longrightarrow P \oplus P_{1} \longrightarrow Q \oplus P_{1} \longrightarrow 0,
$$


qui induit un morphisme $T_{P} \times T_{P_{1}} \rightarrow T$ lequel se factorise en

$$
T_{P} \times T_{P_{1}} \longrightarrow T_{P} \longrightarrow T
$$

où la première flèche est la projection naturelle et la deuxième le morphisme induit par (3.1). On a un isomorphisme naturel $\mathcal{D}_{T_{P} \times T_{P_{1}}} \stackrel{\sim}{\rightarrow} \mathcal{D}_{T_{P}} \times \mathcal{D}_{T_{P_{1}}}$ et le morphisme $\mathcal{D}_{T_{P}} \times \mathcal{D}_{T_{P_{1}}} \rightarrow \mathcal{D}_{T_{P}}$ induit par la première flèche de (3.39) n'est autre que la projection naturelle. On a donc

$$
\operatorname{Coker}\left(\mathcal{D}_{T_{P} \times T_{P_{1}}} \rightarrow \mathcal{D}_{T}\right)=\operatorname{Coker}\left(\mathcal{D}_{T_{P}} \rightarrow \mathcal{D}_{T}\right) .
$$

Par ailleurs si

$$
0 \longrightarrow X(T) \longrightarrow P^{\prime} \longrightarrow Q^{\prime} \longrightarrow 0 \text {. }
$$

est une autre résolution flasque de $X(T)$, par [CTSa, lemme 5], les $G$-modules $Q$ et $Q^{\prime}$ sont isomorphes après addition de $G$-modules de permutation convenables.

On note $\mathcal{K}_{T}$ le cardinal du conoyau de la flèche $\mathcal{D}_{T_{P}} \longrightarrow \mathcal{D}_{T}$.

\section{Proposition 3.36}

On a $\mathcal{K}_{T}=1$ dans les cas suivants :

- $T$ vérifie l'approximation faible (i.e. $A(T)=0$ )

- T est anisotrope,

- T est déployé par une extension dans laquelle $k$ est algébriquement clos.

Démonstration: Rappelons que l'on désigne par $L$ une extension galoisienne finie déployant $T$, et que son groupe de Galois est noté $G$.

Si $A(T)=0$, le lemme 3.32 montre que le morphisme

$$
T_{P}\left(\mathbf{A}_{K}\right) \longrightarrow T\left(\mathbf{A}_{K}\right)
$$

est surjectif. Ainsi $\mathcal{K}_{T}=1$.

Si $T$ est anisotrope, $X(T)^{G}=0$, donc $\mathcal{D}_{T}=0$, d'où le résultat.

Supposons $T$ déployé par une extension dans laquelle $k$ est algébriquement clos. Alors $k$ est encore algébriquement clos dans la clôture galoisienne d'une telle extension. On peut ainsi supposer que $k$ est algébriquement clos dans $L$. D'après le corollaire 3.21 on a $\mathrm{C}_{T}=0$, i.e.

$$
\mathcal{D}_{T}=\left(X(T)^{G}\right)^{\vee},
$$

et de même

$$
\mathcal{D}_{T_{P}}=\left(P^{G}\right)^{\vee} .
$$

Mais, comme déjà vu dans la preuve du lemme 3.35, la flèche

$$
\left(P^{G}\right)^{\vee} \rightarrow\left(X(T)^{G}\right)^{\vee}
$$


est surjective, d'où le résultat.

Remarque 3.37: Le cas où $A(T)=0$ se produit en particulier quand $T$ est quasi-déployé. Plus généralement, d'après la suite exacte (3.14), il se produit si $H^{1}(G, Q)=0$. D'après [CTSa, Corollaire 2] et la dualité de Nakayama, ceci est vérifié en particulier quand $T$ est déployé par une extension métacyclique ${ }^{3}$ de $K$.

Colliot-Thélène et Suresh ont exhibé dans [CTSu] un exemple de tore algébrique $T$ ne vérifiant pas $\mathcal{K}_{T}=1$, que nous décrivons à présent. Soit $G=\mathbf{Z} / 2 \times \mathbf{Z} / 2$ le groupe de Klein, $\tau$ et $\sigma$ des éléments de $G$ tels que $G$ est engendré par $\tau$ et $\sigma$. On note $I_{G}$ le noyau de l'augmentation $\mathbf{Z}[G] \rightarrow \mathbf{Z}$ et $N_{G}$ l'élément $\sum_{g \in G} g$ de $\mathbf{Z}[G]$. Soit $N$ le sous- $G$-module de $\mathbf{Z}[G]$ défini par

$$
N=\left\{n \in \mathbf{Z}[G], \quad \exists m \in I_{G}, \quad \sigma . n-n=m+\tau m \text { et } \tau . n-n=m+\sigma m\right\}
$$

Soit $L / K$ est une extension galoisienne de groupe $G$ et $T$ le tore algébrique sur $K$ dont le module des caractères est $N^{\vee}$. Alors $T$ s'identifie à un sous-tore de $\operatorname{Res}_{L / K} \mathbf{G}_{m}$, et $T(K)$ s'identifie à un sous-groupe de $\operatorname{Res}_{L / K} \mathbf{G}_{m}(K)=L^{\times}$, plus précisément

$T(K)=\left\{y \in L^{\times}, \quad \exists x \in L^{\times}, \quad N_{L / K}(x)=1,{ }^{\sigma} y y^{-1}=x^{\tau} x\right.$ et $\left.{ }^{\tau} y y^{-1}=x^{\sigma} x\right\}$.

\section{Théorème 3.38 (Colliot-Thélène, Suresh)}

Soit $k$ un corps fini de caractéristique différente de 2 dans lequel -1 est un carré et $K=k(t)$ le corps des fractions rationnelles en une indéterminée sur $k$. Soit u un élément de $k$ qui n'est pas un carré. Soit $L$ l'extension $K(\sqrt{u}, \sqrt{t})$. $C$ 'est une extension galoisienne de $K$ de groupe $G$. On note $\sigma$ (respectivement $\tau$ ) le générateur du groupe de Galois de $K(\sqrt{u}) / K$ (respectivement $K(\sqrt{t}) / K)$. Soit $T$ le tore algébrique sur $K$ de module de cocaractères $N$. Alors $\mathcal{K}_{T}$ est différent de 1.

La démonstration utilise une résolution flasque explicite de $T$, et la proposition suivante, que nous utiliserons à la sous-section 4.2.2 pour montrer que sur certaines variétés toriques, les hauteurs logarithmique canoniques locales peuvent ne pas être à valeurs entières.

\section{Proposition 3.39}

On conserve les notations du théorème 3.38.

1. On a $\sqrt{u} \sqrt{t} \in T(K)$.

\footnotetext{
${ }^{3}$ dont le groupe de Galois est à Sylow cycliques
} 
2. Soit $v$ la place de $K$ d'uniformisante $t, v$ l'unique place de $L$ divisant $v$ et $G_{v}$ son groupe de décomposition. Alors $G_{v}=G, v$ est ramifiée, et l'indice de ramification est 2.

3. On a

$$
\operatorname{deg}_{T, L, \mathcal{V}}(\sqrt{u} \sqrt{t})=N_{G}
$$

et

$$
\left(X(T)^{\vee}\right)^{G_{v}}=\boldsymbol{Z} N_{G}
$$

Démonstration : Soit $i \in k$ tel que $i^{2}=-1$. Alors $N_{L / K} i=i^{4}=1$. Or on a

$$
\sigma(\sqrt{u} \sqrt{t})(\sqrt{u} \sqrt{t})^{-1}=-(\sqrt{u} \sqrt{t})(\sqrt{u} \sqrt{t})^{-1}=-1=i^{2}=i^{\tau} i
$$

et

$$
\tau(\sqrt{u} \sqrt{t})(\sqrt{u} \sqrt{t})^{-1}=-(\sqrt{u} \sqrt{t})(\sqrt{u} \sqrt{t})^{-1}=-1=i^{2}=i^{\sigma} i .
$$

Ainsi, d'après (3.47), $\sqrt{u} \sqrt{t}$ est dans $T(K)$, ce qui montre le premier point. Le deuxième point est immédiat.

Pour le troisième point, on note qu'on a le diagramme commutatif suivant

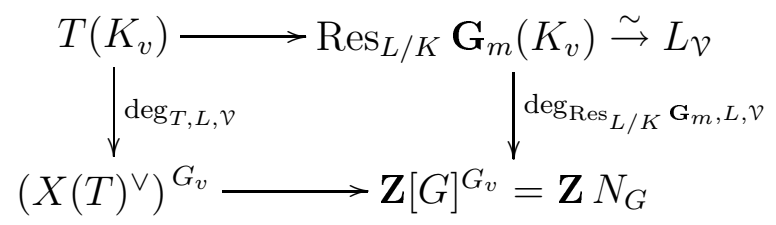

dont les flèches horizontales sont injectives.

Pour tout $y \in L_{v}^{\times}$, on a

$$
\operatorname{deg}_{\operatorname{Res}_{L / K} \mathbf{G}_{m}, L, \mathcal{V}}(y)=\mathcal{V}(y) N_{G} .
$$

Ainsi

$$
\operatorname{deg}_{T, L, \mathcal{V}}(\sqrt{u} \sqrt{t})=\operatorname{deg}_{\operatorname{Res}_{L / K} \mathbf{G}_{m}, L, \mathcal{V}}(\sqrt{u} \sqrt{t})=\mathcal{V}(\sqrt{u} \sqrt{t}) N_{G}=N_{G} .
$$

On en déduit aussitôt que $\left(X(T)^{\vee}\right)^{G_{v}}=\mathbf{Z} N_{G}$.

Comme le $G$-module $P$ apparaissant dans (3.1) est de permutation, le début de la suite exacte longue de cohomologie tirée de (3.1) s'écrit

$$
0 \longrightarrow X(T)^{G} \longrightarrow P^{G} \longrightarrow Q^{G} \longrightarrow H^{1}(G, X(T)) \longrightarrow 0 .
$$


L'homologie du complexe

$$
\left(Q^{G}\right)^{\vee} \rightarrow\left(P^{G}\right)^{\vee} \rightarrow\left(X(T)^{G}\right)^{\vee}
$$

est donc de cardinal $\left[H^{1}(G, X(T))\right]$.

Le lemme suivant est utilisé dans le calcul du terme principal de la fonction zêta des hauteurs dans le cas fonctionnel.

\section{Lemme 3.40}

L'homologie du complexe

$$
\mathcal{D}_{T_{Q}} \longrightarrow \mathcal{D}_{T_{P}} \longrightarrow \mathcal{D}_{T}
$$

est de cardinal

$$
\frac{\left[H^{1}(G, X(T))\right]\left[\mathcal{C}_{T_{Q}}\right]\left[\mathcal{C}_{T}\right] \mathcal{K}_{T}}{\left[\mathcal{C}_{T_{P}}\right]} .
$$

Démonstration: Notons $\mathcal{H}$ ce cardinal. On a le diagramme commutatif suivant

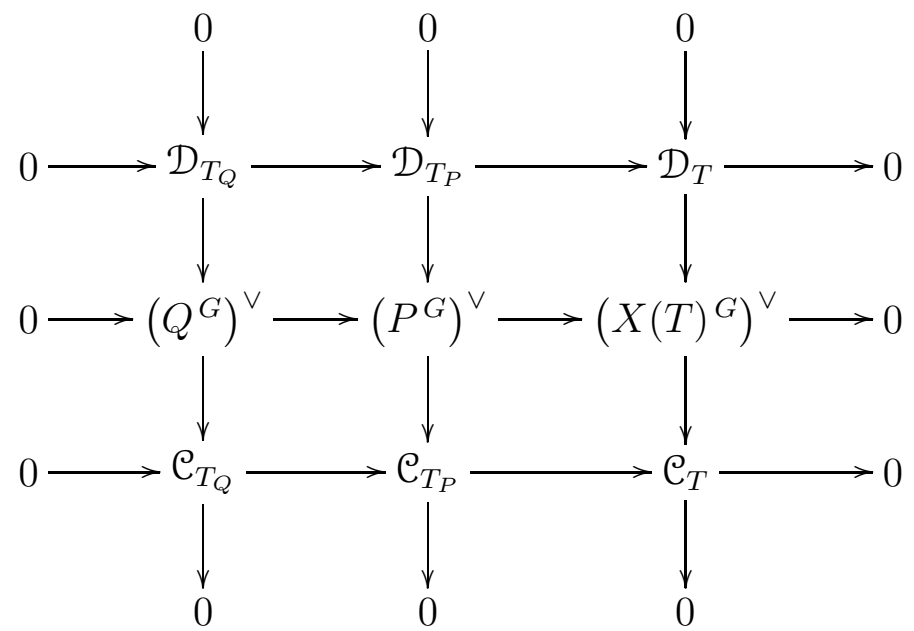

où les verticales sont exactes et les horizontales sont des complexes, en d'autres termes on a une suite exacte de complexes. Par ailleurs les complexes sont exacts en : $\mathcal{D}_{T_{Q}},\left(Q^{G}\right)^{\vee},\left(X(T)^{G}\right)^{\vee}$ et $\mathcal{C}_{T}$. Les caractéristiques d'Euler-Poincaré de ces complexes sont alors, de haut en bas : $\frac{\mathcal{K}_{T}}{\mathcal{H}}, \frac{1}{\left[H^{1}(G, X(T))\right]}$ et $\frac{\left[\mathfrak{C}_{T_{Q}}\right]\left[\mathfrak{e}_{T}\right]}{\left[\mathfrak{e}_{T_{P}}\right]}$. Comme on a une suite exacte de complexes, on en déduit la relation

$$
\frac{\mathcal{H}}{\mathcal{K}_{T}} \frac{1}{\left[H^{1}(G, X(T))\right]} \frac{\left[\mathcal{C}_{T_{P}}\right]}{\left[\mathcal{C}_{T_{Q}}\right]\left[\mathcal{C}_{T}\right]}=1,
$$

d'où le résultat. 


\subsection{Mesure adélique et nombre de Tamagawa d'un tore algébrique}

Si $X$ est une variété algébrique lisse définie sur un corps global $K$, on a déjà rappelé qu'une métrisation du faisceau anticanonique de $X$ permet, pour tout $v \in \mathcal{P}_{K}$, de construire une mesure $\omega_{X, v}$ sur l'espace analytique $X\left(K_{v}\right)$. Rappelons aussi que tout choix d'une section globale $\omega$ partout non nulle du faisceau anticanonique en fournit une métrisation par la formule

$$
\forall x \in X\left(K_{v}\right), \quad \forall s \in \omega_{X}^{-1}(x), \quad\|s\|_{v}=\frac{|s(x)|_{v}}{|\omega(x)|_{v}},
$$

le choix de la valeur absolue $|.|_{v}$ sur $\omega_{X}^{-1}(x)$ étant arbitraire. Si $X=G$ est un groupe algébrique et $\omega$ est de plus choisie $G$-invariante à gauche, les mesures locales obtenues sont des mesures de Haar à gauche.

Ono, dans l'article [On2], définit le nombre de Tamagawa d'un tore algébrique défini sur un corps global. Dans [On3], il établit une relation simple entre ce nombre de Tamagawa et certains invariants de type cohomologique du tore (cf. le théorème 3.41 ci-dessous), montrant en particulier la rationalité du nombre de Tamagawa (chose nullement évidente sur la définition initiale). Cette relation joue un rôle important dans l'interprétation du terme principal des fonctions zêta des hauteurs des variétés toriques, dans le cas arithmétique comme dans le cas fonctionnel. Cependant, dans le cas des corps de fonctions, il s'est avéré que la définition du nombre de Tamagawa d'un tore algébrique donnée dans [On2] était incompatible avec la relation du théorème 3.41. Par la suite Oesterlé a montré dans [Oe] qu'en introduisant dans la définition d'Ono un facteur correctif égal au cardinal du conoyau du degré (ce que nous avons noté $\mathcal{C}_{T}$ ), la relation du théorème 3.41 devenait correcte. Nous rappelons dans cette section la construction du nombre de Tamagawa d'un tore algébrique, et le résultat principal de [On3].

Soit $T$ un tore algébrique de dimension $d$, défini sur un corps global $K$. Soit $\Omega_{T}$ une $d$-forme différentielle $K$-rationnelle $T$-invariante sur $T$ (une telle forme est uniquement déterminée à multiplication par un élément de $K^{\times}$ près). Cette forme induit donc pour tout $v \in \mathcal{P}_{K}$ une mesure de Haar $\omega_{T, v}$ sur $T\left(K_{v}\right)$.

On a alors pour presque toute place finie $v$ la relation

$$
\int_{T\left(\mathcal{O}_{v}\right)} \omega_{T, v}=\frac{1}{L_{v}(1, X(T))} .
$$

Posons, si $v$ est finie,

$$
d \mu_{v}=\frac{1}{L_{v}(1, X(T))} \omega_{T, v}
$$


et si $v$ est archimédienne

$$
d \mu_{v}=\omega_{T, v}
$$

On aura alors

$$
\int_{T\left(\mathcal{O}_{v}\right)} d \mu_{v}=1
$$

pour presque tout $v$.

On peut alors définir une mesure de Haar $\omega_{T}$ sur $T\left(\mathbf{A}_{K}\right)$ en posant

$$
\omega_{T}=c_{K, \operatorname{dim}(T)} \prod_{v \in \mathcal{P}_{K}} d \mu_{v}
$$

(cf (2.10) pour la définition de $\left.c_{K, \operatorname{dim}(T)}\right)$.

Si la forme $\Omega_{T}$ est changée en $\lambda \Omega_{T}$, où $\lambda \in K^{\times}, d \mu_{v}$ est changée en $|\lambda|_{v} d \mu_{v}$ pour tout $v$. Ainsi, par la formule du produit, $\omega_{T}$ ne dépend pas du choix de la forme $\Omega_{T}$.

Notons qu'en particulier

$$
\int_{\mathbf{K}(T)} \omega_{T}=c_{K, \operatorname{dim}(T)} \prod_{v} \int_{T\left(\mathcal{O}_{v}\right)} d \mu_{v}
$$

est non nul.

À partir de $\omega_{T}$, on construit une mesure de Haar sur $T\left(\mathbf{A}_{K}\right)^{1} / T(K)$ de la manière suivante.

Dans le cas arithmétique, soit $d t$ la mesure de Lebesgue sur $\left(X(T)^{G}\right)_{\mathbf{R}}^{\vee}$, normalisée par le réseau $\left(X(T)^{G}\right)^{\vee}$. Rappelons que $\operatorname{deg}_{T}$ induit un isomorphisme de groupes topologiques

$$
\operatorname{deg}_{T}: T\left(\mathbf{A}_{K}\right) / T\left(\mathbf{A}_{K}\right)^{1} \stackrel{\sim}{\longrightarrow}\left(X(T)^{G}\right)_{\mathbf{R}}^{\vee} .
$$

Soit $\widetilde{\omega_{T}}$ la mesure quotient sur $T\left(\mathbf{A}_{K}\right) / T(K)$ induite par $\omega_{T}, T(K)$ étant muni de la mesure discrète. Soit $\omega_{T}^{1}$ la mesure sur $T\left(\mathbf{A}_{K}\right)^{1} / T(K)$ définie par la relation

$$
\widetilde{\omega_{T}}=\omega_{T}^{1} \cdot\left(\operatorname{deg}_{T}^{-1}\right)_{*}(d t)
$$

Dans le cas fonctionnel, comme $T\left(\mathbf{A}_{K}\right)^{1}$ est ouvert dans $T\left(\mathbf{A}_{K}\right)$, la restriction de $\omega_{T}$ à $T\left(\mathbf{A}_{K}\right)^{1}$ fournit une mesure de Haar sur $T\left(\mathbf{A}_{K}\right)^{1}$. Soit $\omega_{T}^{1}$ la mesure quotient sur $T\left(\mathbf{A}_{K}\right)^{1} / T(K)$.

On pose, dans le cas arithmétique,

$$
b(T)=\int_{T\left(\mathbf{A}_{K}\right)^{1} / T(K)} \omega_{T}^{1}
$$


et dans le cas fonctionnel

$$
b(T)=\log \left(q_{K}\right)^{-r g\left(X(T)^{G}\right)} \int_{T\left(\mathbf{A}_{K}\right)^{1} / T(K)} \omega_{T}^{1} .
$$

(rappelons que $T\left(\mathbf{A}_{K}\right)^{1} / T(K)$ est compact).

On pose alors, suivant Ono,

$$
\tau(T)=\frac{b(T)}{\ell(X(T))}
$$

dans le cas arithmétique et, suivant Oesterlé ([Oe, I.5.9 et 5.12.]),

$$
\tau(T)=\frac{1}{\left[\mathcal{C}_{T}\right]} \frac{b(T)}{\ell(X(T))} .
$$

dans le cas fonctionnel. Le nombre $\tau(T)$ est appelé nombre de Tamagawa du tore algébrique $T$.

L'objet de l'article [On3], corrigé par Oesterlé dans le cas fonctionnel, est la démonstration du

\section{Théorème 3.41 (Ono, Oesterlé)}

On a la relation

$$
\tau(T)=\frac{\left[H^{1}(G, X(T))\right]}{[\amalg(T)]} .
$$

Ce résultat, comme déjà indiqué, sera utile lors de l'interprétation du terme principal de la fonction zêta des hauteurs.

\section{Hauteurs sur une variété torique et fonction zêta associée}

\subsection{Géométrie des variétés toriques}

\subsubsection{Variétés toriques déployées}

Nous rappelons la construction des variétés toriques déployées. On renvoie aux références classiques sur les variétés toriques, comme [Fu] et [Od] pour plus de détails et la preuve des assertions qui suivent.

Soit $M$ un $\mathbf{Z}$-module libre de rang fini et $\Lambda$ un cône de $M_{\mathbf{R}}$. L'intérieur relatif de $\Lambda$ sera noté intrel $(\Lambda)$. Le cône dual de $\Lambda$ est noté $\Lambda^{\vee}$ et est défini par

$$
\Lambda \stackrel{\text { déf }}{=}\left\{y \in M_{\mathbf{R}}^{\vee}, \quad \forall x \in \Lambda, \quad\langle y, x\rangle \geqslant 0\right\}
$$


Le cône $\Lambda$ dit polyédral rationnel s'il est engendré par un ensemble fini d'éléments de $M$. Si $\Lambda$ est un cône polyédral rationnel, son dual est encore polyédral rationnel. Le cône $\Lambda$ est dit strictement convexe si

$$
\Lambda \cap-\Lambda=\{0\}
$$

Remarquons que $\Lambda$ est strictement convexe si et seulement si $\Lambda^{\vee}$ est d'intérieur non vide.

On fixe un corps de base $L$. Soit $M$ un Z-module libre de rang fini et $T_{L}=\operatorname{Spec}(L[M])$, c'est-à-dire que $T_{L}$ est le tore algébrique défini sur $L$ ayant pour groupe de caractères $M$.

Soit $\sigma$ un cône polyédral rationnel strictement convexe de $M_{\mathbf{R}}^{\vee}$. À un tel cône est associé une variété affine normale définie sur $L$

$$
X_{\sigma, L}=\operatorname{Spec}\left(L\left[\sigma^{\vee} \cap M\right]\right),
$$

munie naturellement d'une action de $T_{L}$.

Un éventail de $M^{\vee}$ est un ensemble fini $\Sigma$ de cônes polyédraux rationnels strictement convexes de $M_{\mathbf{R}}^{\vee}$, vérifiant les conditions suivantes :

- toute face d'un cône de $\Sigma$ est un cône de $\Sigma$;

- l'intersection de deux cônes de $\Sigma$ est une face de chacun des deux cônes.

Si $\sigma$ et $\sigma^{\prime}$ sont deux cônes de $\Sigma$, les inclusions $\sigma \cap \sigma^{\prime} \subset \sigma$ et $\sigma \cap \sigma^{\prime} \subset \sigma^{\prime}$ induisent des immersions ouvertes $X_{\sigma \cap \sigma^{\prime}, L} \hookrightarrow X_{\sigma, L}$ et $X_{\sigma \cap \sigma^{\prime}, L} \hookrightarrow X_{\sigma^{\prime}, L}$, qui sont compatibles avec les actions de $T_{L}$. Ceci donne un procédé de recollement des variétés $X_{\sigma, L}$ pour $\sigma$ décrivant les cônes de $\Sigma$, lequel procédé est compatible aux actions de $T_{L}$, et permet de construire une variété normale $X_{\Sigma, L}$, munie d'une action de $T_{L}$ : c'est la variété torique (définie sur $L$ ) associée à l'éventail $\Sigma$.

Un éventail $\Sigma$ est dit non dégénéré si ses cônes ne sont pas inclus dans un sous-espace strict de $M_{\mathbf{R}}^{\vee}$.

Un éventail $\Sigma$ est dit régulier si tout cône de $\Sigma$ est engendré par une partie d'une $\mathbf{Z}$-base de $M^{\vee}$, et complet si les cônes de $\Sigma$ recouvrent $M_{\mathbf{R}}^{\vee}$. Un éventail $\Sigma$ est régulier (respectivement complet) si et seulement si la variété $X_{\Sigma, L}$ est lisse (respectivement complète).

Un éventail $\Sigma$ est dit projectif si la variété $X_{\Sigma, L}$ est projective.

Les rayons de $\Sigma$ sont les cônes de $\Sigma$ de dimension 1 . On note $\Sigma(1)$ l'ensemble des rayons de $\Sigma$. Pour $\alpha \in \Sigma(1)$, on note $\rho_{\alpha}$ l'élément de $M^{\vee}$ qui engendre le monoïde $\alpha \cap M^{\vee}$. Pour tout cône $\sigma$ de $\Sigma$ nous notons

$$
\sigma(1)=\{\alpha \in \Sigma(1), \alpha \subset \sigma\}
$$

$($ ainsi $\{0\}(1)=\varnothing)$. 
L'application qui à un élément $\alpha$ de $\Sigma(1)$ associe l'adhérence dans $X_{\Sigma, L}$ de la $T_{L}$-orbite fermée de $X_{\alpha, L}$ définit une bijection de $\Sigma(1)$ sur l'ensemble des diviseurs irréductibles de $X_{\Sigma, L}$ contenu dans le bord $X_{\Sigma, L} \backslash T_{L}$. Pour tout rayon $\alpha$, on note $D_{\alpha}$ le diviseur irréductible ainsi associé à $\alpha$; c'est un diviseur $T_{L}$-invariant. On note $P_{\Sigma}$ le $\mathbf{Z}$-module libre de base $\left(D_{\alpha}\right)_{\alpha \in \Sigma(1)}$; ce n'est autre que le groupe des diviseurs de Weil $T_{L}$-invariants sur $X_{\Sigma, L}$.

On note également $\mathrm{PL}(\Sigma)$ le groupe des applications $\Sigma$-linéaires par morceaux sur $M^{\vee}$, c'est-à-dire les applications $\varphi: M^{\vee} \rightarrow \mathbf{Z}$ telles que la restriction de $\varphi$ à $\sigma \cap M^{\vee}$ est linéaire pour tout cône $\sigma$ de $\Sigma$.

On suppose à présent $\Sigma$ régulier et non dégénéré. L'application

$$
\varphi \mapsto \sum_{\alpha \in \Sigma(1)} \varphi\left(\rho_{\alpha}\right) D_{\alpha}
$$

est alors un isomorphisme de groupes qui permet d'identifier $\operatorname{PL}(\Sigma)$ à $P_{\Sigma}$. Par la suite nous utiliserons souvent cette identification.

Comme le groupe de Picard de $T_{L}$ est trivial, l'application qui à un élément de $P_{\Sigma}$ associe sa classe dans $\operatorname{Pic}\left(X_{\Sigma, L}\right)$, induit une suite exacte

$$
0 \longrightarrow L\left[T_{L}\right]^{\times} / L^{\times} \longrightarrow P_{\Sigma} \longrightarrow \operatorname{Pic}\left(X_{\Sigma, L}\right) \longrightarrow 0,
$$

où la flèche $L\left[T_{L}\right]^{\times} / L^{\times} \longrightarrow P_{\Sigma}$ est induite par l'application qui à une fonction rationnelle associe son diviseur. D'après le lemme de Rosenlicht, $L\left[T_{M}\right]^{\times} / L^{\times}$ est isomorphe au groupe des caractères de $T_{M}$, c'est-à-dire $M$, d'où la suite exacte

$$
0 \longrightarrow M \stackrel{\gamma}{\longrightarrow} P_{\Sigma} \longrightarrow \operatorname{Pic}\left(X_{\Sigma, L}\right) \longrightarrow 0 .
$$

Pour $m \in M$, on a

$$
\gamma(m)=\sum_{\alpha \in \Sigma(1)}\left\langle m, \rho_{\alpha}\right\rangle D_{\alpha}
$$

En outre $\operatorname{Pic}\left(X_{\Sigma, L}\right)$ est un $\mathbf{Z}$-module libre de rang fini.

On a le résultat suivant :

\section{Proposition 4.1}

La classe dans $\operatorname{Pic}\left(X_{\Sigma, L}\right)$ du faisceau anticanonique de $X_{\Sigma, L}$ coïncide avec la classe du diviseur $\sum_{\alpha \in \Sigma(1)} D_{\alpha}$.

\subsubsection{Variétés toriques non déployées}

Nous rappelons à présent la construction des variétés toriques non nécessairement déployées. On se limitera au cas des variétés toriques projectives et lisses. On se donne un tore algébrique $T$ défini sur un corps $K$. Soit $L / K$ 
une extension finie galoisienne de groupe $G$ déployant $T$. Soit $\Sigma \subset X(T)_{\mathbf{R}}^{\vee}$ un éventail projectif et lisse et $X_{\Sigma, L}$ la variété projective et lisse associée.

On suppose en outre que l'action de $G$ sur $X(T)_{\mathbf{R}}^{\vee}$ préserve les cônes de $\Sigma$ (on dira alors que $\Sigma$ est un $G$-éventail ${ }^{4}$ ). Alors $G$ agit aussi sur le schéma projectif $X_{\Sigma, L}$, et on peut donc considérer le quotient de $X_{\Sigma, L}$ par $G$. Ce quotient est une variété $X_{\Sigma}$ définie sur $K$, qui est une compactification équivariante projective et lisse de $T$ (cf. [Vo, §1]).

Le $\mathbf{Z}$-module $P_{\Sigma}$ est alors muni naturellement d'une action de $G$, et le $G$-module résultant est un $G$-module de permutation. Par ailleurs, l'action de $G$ sur les cônes de $\sigma$ induit naturellement une action de $G$ sur $\operatorname{PL}(\Sigma)$ et l'isomorphisme (4.5) est un isomorphisme de $G$-modules.

Nous notons $\Sigma(1) / G$ l'ensemble des orbites de $\Sigma(1)$ sous l'action de $G$. Pour chaque $\alpha \in \Sigma(1) / G$ nous choisissons arbitrairement un élément de $\alpha$, nous notons $\rho_{\alpha}$ le générateur de cet élément ainsi que $G_{\alpha}$ le stabilisateur de $\rho_{\alpha}$, de sorte que le $G$-ensemble $\alpha$ s'identifie à $G / G_{\alpha}$. On déduit de ces choix un isomorphisme

$$
P_{\Sigma} \stackrel{\sim}{\longrightarrow} \bigoplus_{\alpha \in \Sigma(1) / G} \mathbf{Z}\left[G / G_{\alpha}\right]
$$

d'où un isomorphismes de $K$-tores

$$
T_{P_{\Sigma}} \stackrel{\sim}{\longrightarrow} \prod_{\alpha \in \Sigma(1) / G} \operatorname{Res}_{L}^{G_{\alpha} / K} \mathbf{G}_{m} .
$$

Pour $\alpha \in \Sigma(1) / G$, on notera $K_{\alpha}$ le corps $L^{G_{\alpha}}$, et $i_{\alpha}$ la projection $G$-équivariante de $P_{\Sigma}$ sur $\mathbf{Z}\left[G / G_{\alpha}\right]$ induite par l'isomorphisme (4.9). Le morphisme de $K$ tores associé est l'injection $\operatorname{Res}_{K_{\alpha} / K} \mathbf{G}_{m} \rightarrow T_{P_{\Sigma}}$ induite par l'isomorphisme (4.10).

Pour $\alpha \in \Sigma(1) / G$, on note $D_{\alpha}=\sum_{\beta \in \alpha} D_{\beta}$. Ainsi $\left(D_{\alpha}\right)_{\alpha \in \Sigma(1) / G}$ est une base de $P_{\Sigma}^{G}$. On note $\left(D_{\alpha}^{\vee}\right)_{\alpha \in \Sigma(1) / G}$ sa base duale.

La suite exacte de $\mathbf{Z}$-modules libres de rang fini

$$
0 \longrightarrow X(T) \stackrel{\gamma}{\longrightarrow} P_{\Sigma} \longrightarrow \operatorname{Pic}\left(X_{\Sigma, L}\right) \longrightarrow 0
$$

est une suite exacte de $G$-modules. Il en résulte par dualité une suite exacte de tores algébriques

$$
0 \longrightarrow T_{\mathrm{NS}} \longrightarrow T_{P_{\Sigma}} \longrightarrow T \longrightarrow 0
$$

\footnotetext{
${ }^{4}$ On peut montrer qu'un $G$-éventail projectif et lisse de $X(T)_{\mathbf{R}}^{\vee}$ existe toujours, cf. [CTHaSk].
} 
Comme $P_{\Sigma}$ est un $G$-module de permutation, on a $H^{1}\left(G, P_{\Sigma}\right)=0$. Ainsi, en prenant les $G$-invariants dans (4.11), on obtient la suite exacte

$$
0 \longrightarrow X(T)^{G} \stackrel{\gamma}{\longrightarrow} P_{\Sigma}^{G} \longrightarrow \operatorname{Pic}\left(X_{\Sigma}\right) \longrightarrow H^{1}(G, X(T)) \longrightarrow 0 .
$$

D'après (4.8), on a

$$
\forall m \in X(T)^{G}, \quad \gamma(m)=\sum_{\alpha \in \Sigma(1) / G}\left\langle m, \rho_{\alpha}\right\rangle D_{\alpha}
$$

On en déduit qu'on a

$$
\forall \alpha \in \Sigma(1) / G, \quad \gamma^{\vee}\left(D_{\alpha}^{\vee}\right)=\rho_{\alpha}
$$

On a en outre le résultat suivant :

\section{Lemme 4.2}

$\operatorname{Pic}\left(X_{\Sigma, L}\right)$ est un $G$-module flasque.

En d'autres termes, la suite exacte (4.11) est une résolution flasque de $X(T)$.

Démonstration: Dans le cas où $K$ est de caractéristique zéro, ce résultat est contenu dans la preuve de la proposition 6 (page 189) de [CTSa]. Le cas où $K$ est de caractéristique non nulle en découle. Choisissons en effet un corps $K^{\prime}$ de caractéristique 0 tel qu'il existe une extension galoisienne $L^{\prime}$ de $K^{\prime}$ de groupe $G$ (rappelons qu'un procédé classique pour construire une telle extension $L^{\prime} / K^{\prime}$ est de plonger $G$ dans le groupe symétrique $\mathfrak{S}_{n}$ pour $n$ convenable, et de considérer l'extension $\mathbf{Q}\left(T_{1}, \ldots, T_{n}\right) / \mathbf{Q}\left(\sigma_{1}, \ldots, \sigma_{n}\right)$ où les $T_{i}$ sont des indéterminées et les $\sigma_{i}$ les polynômes symétriques élémentaires en ces indéterminées ; cette extension est galoisienne de groupe $\mathfrak{S}_{n}$, et l'extension $\mathbf{Q}\left(T_{1}, \ldots, T_{n}\right) / \mathbf{Q}\left(T_{1}, \ldots, T_{n}\right)^{G}$ fournit l'extension cherchée).

Il existe donc un tore algébrique $T_{P}$ défini sur $K^{\prime}$ et déployé par $L^{\prime}$, et une compactification lisse et projective $X_{\Sigma, K^{\prime}}$ de $T_{P}$, telle que les $G$-modules $\operatorname{Pic}\left(X_{\Sigma, L^{\prime}}\right)$ et $\operatorname{Pic}\left(X_{\Sigma, L}\right)$ sont isomorphe, et d'après [CTSa] $\operatorname{Pic}\left(X_{\Sigma, L^{\prime}}\right)$ est flasque.

\subsection{Hauteurs sur une variété torique}

\subsubsection{Hauteurs locales}

On conserve les objets et notations introduits à la section précédente, et on suppose désormais que $K$ un corps global.

On a un accouplement naturel

$$
\langle,\rangle_{\Sigma}: \operatorname{PL}(\Sigma) \times X(T)^{\vee} \longrightarrow \mathbf{Z}
$$


linéaire en le premier facteur, et qui prolonge l'accouplement naturel

$$
\langle,\rangle: X(T) \times X(T)^{\vee} \longrightarrow \mathbf{Z},
$$

c'est-à-dire que le diagramme

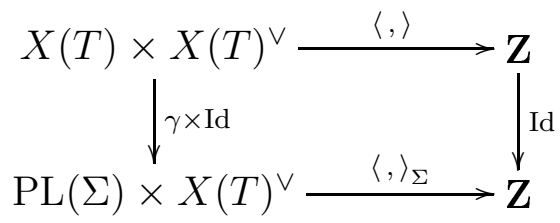

est commutatif.

Soit $v$ une place de $K$. On va définir un système de hauteurs locales en $v$. On note $\mathcal{V}$ une place de $L$ divisant $v$ et $G_{v}$ le groupe de décomposition correspondant.

Supposons tout d'abord $v$ finie. Notons $e_{v}$ l'indice de ramification de $v$ dans l'extension $L / K$. On définit un «produit d'intersection local»

$$
\langle,\rangle_{\Sigma, v}: \operatorname{PL}(\Sigma)^{G_{v}} \times T\left(K_{v}\right) \longrightarrow \frac{1}{e_{v}} \mathbf{Z}
$$

par la formule

$$
\langle\varphi, t\rangle_{\Sigma, v}=\frac{1}{e_{v}}\left\langle\varphi, \operatorname{deg}_{T, L, v}(t)\right\rangle_{\Sigma}
$$

On vérifie que cette définition ne dépend ni du choix de $\mathcal{V}$, ni du choix de l'extension $L$ déployant $T$. Par linéarité en le premier facteur, on étend $\langle,\rangle_{\Sigma}$ en un accouplement

$$
\langle,\rangle_{\Sigma, v}: \operatorname{PL}(\Sigma)_{\mathbf{C}}^{G_{v}} \times T\left(K_{v}\right) \longrightarrow \mathbf{C}
$$

\section{Lemme 4.3}

Le diagramme

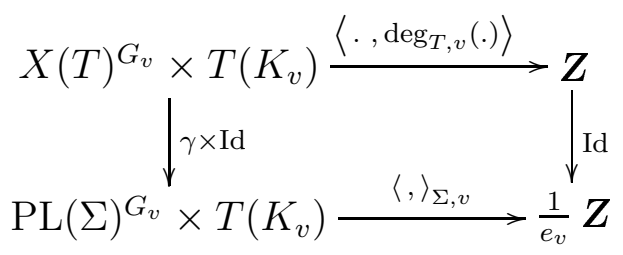

est commutatif 
Démonstration : Soit $m \in X(T)^{G_{v}}$ et $t \in T\left(K_{v}\right)$. On a

$$
\begin{aligned}
\langle\gamma(m), t\rangle_{\Sigma, v} & =\frac{1}{e_{v}}\left\langle\gamma(m), \operatorname{deg}_{T, L, v}(t)\right\rangle_{\Sigma} \\
& =\frac{1}{e_{v}}\left\langle m, \operatorname{deg}_{T, L, \mathcal{V}}(t)\right\rangle \\
& =\frac{1}{e_{v}}\left\langle m, e_{v} \operatorname{deg}_{T, v}(t)\right\rangle \\
& =\left\langle m, \operatorname{deg}_{T, v}(t)\right\rangle .
\end{aligned}
$$

L'égalité (4.9) vient de la commutativité du diagramme (4.3), et l'égalité (4.9) du lemme 3.11.

Supposons à présent $v$ archimédienne. On définit un «produit d'intersection local»

$$
\langle,\rangle_{\Sigma, v}: \operatorname{PL}(\Sigma)^{G_{v}} \times T\left(K_{v}\right) \longrightarrow \mathbf{R}
$$

par la formule

$$
\langle\varphi, t\rangle_{\Sigma, v}=\frac{1}{\left[L_{\mathcal{V}}: K_{v}\right]}\left\langle\varphi, \operatorname{deg}_{T, L, \mathcal{V}}(t)\right\rangle_{\Sigma}
$$

On vérifie que cette définition ne dépend ni du choix de $\mathcal{V}$, ni du choix de l'extension $L$ déployant $T$. Par linéarité en le premier facteur, on étend $\langle,\rangle_{\Sigma, v}$ en un accouplement

$$
\langle,\rangle_{\Sigma, v}: \operatorname{PL}(\Sigma)_{\mathbf{C}}^{G_{v}} \times T\left(K_{v}\right) \longrightarrow \mathbf{C}
$$

\section{Lemme 4.4}

Le diagramme

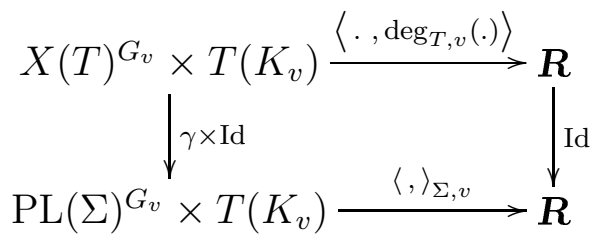

est commutatif.

Démonstration : Soit $m \in X(T)_{v}^{G}$ et $t \in T\left(K_{v}\right)$. On a

$$
\begin{aligned}
\langle\gamma(m), t\rangle_{\Sigma, v} & =\frac{1}{\left[L_{\mathcal{V}}: K_{v}\right]}\left\langle\gamma(m), \operatorname{deg}_{T, L, \mathcal{V}}(t)\right\rangle_{\Sigma} \\
& =\frac{1}{\left[L_{\mathcal{V}}: K_{v}\right]}\left\langle m, \operatorname{deg}_{T, L, \mathcal{V}}(t)\right\rangle \\
& =\frac{1}{\left[L_{\mathcal{V}}: K_{v}\right]}\left\langle m,\left[L_{\mathcal{V}}: K_{v}\right] \operatorname{deg}_{T, v}(t)\right\rangle \\
& =\left\langle m, \operatorname{deg}_{T, v}(t)\right\rangle .
\end{aligned}
$$


La deuxième égalité vient de la commutativité du diagramme (4.3), et la troisième du lemme 3.12 .

Pour toute place $v$ de $K$, on définit alors un système de hauteurs (exponentielles) locales

$$
H_{v}: \begin{aligned}
\operatorname{PL}(\Sigma)_{\mathbf{C}}^{G_{v}} \times T\left(K_{v}\right) & \longrightarrow \mathbf{C} \\
(\varphi, t) & \longmapsto \exp \left[\log \left(q_{v}\right)\langle\varphi, t\rangle_{\Sigma, v}\right]
\end{aligned}
$$

Remarque 4.5 : À la présentation près, le système de hauteurs utilisé est le même que celui décrit dans [BaTs1, Definition 2.1.5]. Notons toutefois que les auteurs de [BaTs1] omettent le facteur $\frac{1}{e_{v}}$ apparaissant dans la définition (4.5) ce qui, pour les places ramifiées, rend leur définition incorrecte au sens où les points 3 et $4 \mathrm{du}$ lemme 4.6 ne sont plus vérifiés.

\section{Lemme 4.6}

Soit $v$ une place de $K, \mathcal{V}$ une place de $L$ divisant $v$, et $G_{v}$ le groupe de décomposition correspondant.

1. Pour tout $\varphi \in \operatorname{PL}(\Sigma)_{\boldsymbol{C}}^{G_{v}}$, la fonction $H_{v}(\varphi,$.$) est invariante sous l'action$ de $T\left(\mathcal{O}_{v}\right)$.

2. Pour tous $\varphi_{1}, \varphi_{2} \in \operatorname{PL}(\Sigma)_{\boldsymbol{C}}^{G}$, on a

$$
H_{v}\left(\varphi_{1}+\varphi_{2}, .\right)=H_{v}\left(\varphi_{1}, .\right) H_{v}\left(\varphi_{2}, .\right) .
$$

3. On identifie $T\left(K_{v}\right)$ à un sous-groupe de $T\left(L_{\mathcal{V}}\right)$. On a alors pour tout $\varphi \in \operatorname{PL}(\Sigma)^{G_{v}}$ et tout $t \in T\left(K_{v}\right)$

$$
H_{\mathcal{V}}(\varphi, t)=H_{v}(\varphi, t)^{\left[L_{v}: K_{v}\right]}
$$

4. Soit $\varphi \in P L(\Sigma)^{G}$. Il existe une unique métrique $v$-adiques $\|.\|_{v}^{\varphi}$ sur $\mathcal{O}_{X_{\Sigma}}(\varphi)$ vérifiant

$$
\forall t \in T\left(K_{v}\right), \quad H_{v}(\varphi, t)=\left(\|\mathbf{1}(t)\|_{v}^{\varphi}\right)^{-1}
$$

où 1 est la fonction régulière sur $X_{\Sigma}$ constante égale à 1 (identifiée à la section rationnelle canonique du fibré $\mathcal{O}_{X_{\Sigma}}(\varphi)$ ).

La métrique $\left(\|.\|_{v}^{\varphi}\right)_{v \in \mathcal{P}_{K}}$ est une métrique adélique sur le fibré en droites $\mathcal{O}_{X_{\Sigma}}(\varphi)$.

Démonstration : (cf. également [BaTs1, Thm 2.1.6]) Les points 1 et 2 découlent immédiatemment de la définition. 
Pour le point 3 , supposons d'abord $v$ finie. D'après la commutativité du diagramme (3.42), on peut écrire, pour $t \in T\left(K_{v}\right)$ et $\varphi \in \operatorname{PL}(\Sigma)^{G_{v}}$,

$$
\begin{aligned}
\log \left(q_{\mathcal{V}}\right)\left\langle\varphi, \operatorname{deg}_{T_{L}, \mathcal{V}}(t)\right\rangle_{\Sigma} & =\log \left(q_{\mathcal{V}}\right)\left\langle\varphi, \operatorname{deg}_{T, L, \mathcal{V}}(t)\right\rangle_{\Sigma} \\
& =\log \left(q_{v}^{\frac{\left[L_{\left.\mathcal{V}: K_{v}\right]}\right.}{e_{v}}}\right)\left\langle\varphi, \operatorname{deg}_{T, L, \mathcal{V}}(t)\right\rangle_{\Sigma} \\
& =\left[L_{\mathcal{V}}: K_{v}\right] \log \left(q_{v}\right)\langle\varphi, t\rangle_{\Sigma, v} .
\end{aligned}
$$

d'où le résultat.

Supposons à présent $v$ archimédienne. D'après la commutativité du diagramme 3.46, on peut écrire pour $t \in T\left(K_{v}\right)$ et $\varphi \in \mathrm{PL}(\Sigma)^{G_{v}}$,

$$
\begin{aligned}
\left\langle\varphi, \operatorname{deg}_{T_{L}, \mathcal{V}}(t)\right\rangle_{\Sigma} & =\left\langle\varphi, \operatorname{deg}_{T, L, \mathcal{V}}(t)\right\rangle_{\Sigma} \\
& =\left[L_{\mathcal{V}}: K_{v}\right] \frac{1}{\left[L_{\mathcal{V}}: K_{v}\right]}\left\langle\varphi, \operatorname{deg}_{T, L, \mathcal{V}}(t)\right\rangle_{\Sigma} \\
& =\left[L_{\mathcal{V}}: K_{v}\right]\langle\varphi, t\rangle_{\Sigma, v}
\end{aligned}
$$

d'où le résultat.

Passons à la démonstration du point 4 .

L'unicité de la métrique vient de la densité de $T\left(K_{v}\right)$ dans $X_{\Sigma}\left(K_{v}\right)$ et de la continuité de l'application $x \mapsto\|s(x)\|_{v, x}$ pour toute métrique $v$-adique $\|.\|_{v} \operatorname{sur} \mathcal{O}_{X_{\Sigma}}(\varphi)$ et toute section locale $s$ de $\mathcal{O}_{X_{\Sigma}}(\varphi)$.

D'après le lemme 2.10 et la relation (4.22), il suffit de montrer l'existence de la métrique dans le cas où le tore $T$ est déployé.

Comme tout fibré en droites sur $X_{\Sigma}$ est le quotient de deux fibrés en droites engendrés par leurs sections, il suffit d'après le lemme 2.9 et (4.21) de montrer l'existence de la métrique pour les $\varphi$ tels que $\mathcal{O}_{X_{\Sigma}}(\varphi)$ est engendré par ses sections.

On note alors $\left(\|.\|_{v}^{\text {st }}\right)_{v \in \mathcal{P}_{K}}$ la métrique adélique standard sur $\mathcal{O}_{X_{\Sigma}}(\varphi)$ (cf. définition 2.8) et on va montrer

$$
\forall v \in \mathcal{P}_{K}, \quad \forall t \in T\left(K_{v}\right), \quad H_{v}(\varphi, t)=\left(\|\mathbf{1}(t)\|_{v}^{\text {st }}\right)^{-1} .
$$

Soit $\left\{m_{0}, \ldots, m_{r}\right\}$ une base de $H^{0}\left(X_{\Sigma}, \mathcal{O}(\varphi)\right)$ constituée de caractères de $T$ (cf. [Fu, Lemma, p. 66]).

Pour $n \in X(T)^{\vee}, \mathcal{O}_{X_{\Sigma}}(\varphi)$ étant engendré par ses sections, on a alors d'après [Fu, Proposition, p. 68]

$$
\varphi(n)=\operatorname{Max}_{i=0, \ldots, r}\left\langle m_{i}, n\right\rangle .
$$

Soit d'abord $v$ une place finie de $K$. Pour $t \in T\left(K_{v}\right)$, on a

$$
\left\langle\varphi, \operatorname{deg}_{T, K, v}(t)\right\rangle_{\Sigma}=\operatorname{Max}_{i=0, \ldots, r}\left\langle m_{i}, \operatorname{deg}_{T, K, v}(t)\right\rangle=\operatorname{Max}_{i=0, \ldots, r} v\left(m_{i}(t)\right)
$$


soit

$$
H_{v}(\varphi, x)=q_{v}^{\left\langle\varphi, \operatorname{deg}_{T, K, v}(t)\right\rangle_{\Sigma}}=\operatorname{Max}_{i=0, \ldots, r} q_{v}^{v\left(m_{i}(t)\right)}=\operatorname{Max}_{i=0, \ldots, r}\left|m_{i}(t)\right|_{v} .
$$

Soit à présent $v$ une place archimédienne. Pour $t \in T\left(K_{v}\right)$, on a

$$
\varphi\left(\operatorname{deg}_{T, K, v}(t)\right)=\operatorname{Max}_{i=0, \ldots, r}\left\langle m_{i}, \operatorname{deg}_{T, K, v}(t)\right\rangle=\underset{i=0, \ldots, r}{\operatorname{Max}} \log \left|m_{i}(t)\right|_{v}
$$

soit

$$
H_{v}(\varphi, t)=\exp \left(\left\langle\varphi, \operatorname{deg}_{T, K, v}(t)\right\rangle_{\Sigma}\right)=\operatorname{Max}_{i=0, \ldots, r}\left|m_{i}(t)\right|_{v} .
$$

Mais par définition de la métrique adélique standard, on a dans tous les cas

$$
\left(\|\mathbf{1}(t)\|_{v}^{\mathrm{st}}\right)^{-1}=\operatorname{Max}_{i=0, \ldots, r}\left|m_{i}(t)\right|_{v}
$$

d'où le résultat.

Remarque 4.7: Pour tout entier $d \geqslant 2$, notons $[d]$ l'endomorphisme de $X_{\Sigma}$ induit par l'endomorphisme $t \mapsto t^{d}$ de $T$. Pour tout $\varphi \in P L(\Sigma)^{G}$, on a alors un isomorphisme canonique

$$
[d]^{*} \mathcal{O}_{X_{\Sigma}}(\varphi) \stackrel{\sim}{\rightarrow} \mathcal{O}_{X_{\Sigma}}(\varphi)^{d} .
$$

Par ailleurs toute métrique $v$-adique sur $\varphi_{v}$ induit naturellement des métriques $\operatorname{sur}[d]^{*} \mathcal{O}_{X_{\Sigma}}(\varphi)$ et $\mathcal{O}_{X_{\Sigma}}(\varphi)^{d}$ respectivement. La métrique $v$-adique $\|.\|_{v}^{\varphi}$ définie ci-dessus est alors la métrique $v$-adique canonique sur $\mathcal{O}_{X_{\Sigma}}(\varphi)$, au sens où c'est l'unique métrique $v$-adique sur $\mathcal{O}_{X_{\Sigma}}(\varphi)$ tel que l'isomorphisme 4.37 soit une isométrie.

\subsubsection{Remarques sur le cas fonctionnel}

Dans le cas fonctionnel, une définition alternative des hauteurs locales nous sera utile. Soit $v$ une place de $K$. L'accouplement $H_{v}$ se réécrit

$$
H_{v}: \begin{aligned}
\operatorname{PL}(\Sigma)_{\mathbf{C}}^{G_{v}} \times T\left(K_{v}\right) & \longrightarrow \mathbf{C} \\
(\varphi, t) & \longmapsto q^{f_{v}\langle\varphi, t\rangle_{\Sigma, v}}
\end{aligned}
$$

Supposons à présent que $v$ vérifie l'hypothèse suivante :

\section{Hypothèse 4.8}

Pour tout $\varphi \in \operatorname{PL}(\Sigma)^{G}$ et pour tout $t \in T\left(K_{v}\right), f_{v}\langle\varphi, t\rangle_{\Sigma, v}$ est entier 
Pour $t \in T\left(K_{v}\right)$ on peut alors étendre par linéarité la fonction

$$
\begin{aligned}
\mathrm{PL}(\Sigma)^{G} & \longrightarrow \mathrm{Z} \\
\varphi & \longmapsto f_{v}\langle\varphi, t\rangle_{\Sigma, v}
\end{aligned}
$$

en une application

$$
\begin{aligned}
\operatorname{PL}(\Sigma)_{\mathbf{C}^{\times}}^{G} & \longrightarrow \mathbf{Z} \otimes \mathbf{C}^{\times}=\mathbf{C}^{\times} \\
\psi & \longmapsto\langle\psi, t\rangle_{\Sigma, v}^{f_{v}}
\end{aligned}
$$

On obtient finalement un accouplement

$$
\begin{aligned}
\mathfrak{H}_{v}: \quad \operatorname{PL}(\Sigma)_{\mathbf{C}^{\times}}^{G} \times T\left(K_{v}\right) & \longrightarrow \mathbf{C}^{\times} \\
(\psi, t) & \longmapsto\langle\psi, t\rangle_{\Sigma, v}^{f_{v}} .
\end{aligned}
$$

Par ailleurs le morphisme du groupe $\mathbf{C}$ vers le groupe $\mathbf{C}^{\times}$donné par $s \mapsto q^{s}$ induit un morphisme

$$
\begin{aligned}
\operatorname{PL}(\Sigma)_{\mathbf{C}}^{G} & \longrightarrow \operatorname{PL}(\Sigma)_{\mathbf{C}^{\times}}^{G} \\
\varphi & \longmapsto q^{\varphi}
\end{aligned}
$$

et on a

$$
\forall \varphi \in \operatorname{PL}(\Sigma)_{\mathbf{C}}^{G}, \quad \forall t \in T\left(K_{v}\right), \quad H_{v}(\varphi, t)=\mathfrak{H}_{v}\left(q^{\varphi}, t\right) .
$$

L'hypothèse 4.8 est vérifiée par exemple si $v$ n'est pas ramifiée. En général, l'hypothèse 4.8 n'est pas toujours vérifiée, comme le montrent le lemme 4.9 cidessous, combiné à la proposition 3.39. Par la suite, pour l'étude de la fonction zêta des hauteurs, on se placera très souvent, par souci de simplification, dans le cas où l'hypothèse 4.8 est vérifiée pour toute place $v$ (ce qui est le cas par exemple si $T$ est déployé par une extension non ramifiée). On expliquera en appendice les modifications techniques nécessaires pour adapter le calcul au cas général.

\section{Lemme 4.9}

Soit $T$ un tore algébrique défini sur un corps de fonctions $K$ et $L$ une extension galoisienne de $K$ de groupe $G$ déployant $T$. Soit $v$ une place de $K$ vérifiant les conditions suivantes :

1. $f_{v}=1$;

2. $v$ est ramifiée dans $L$;

3. $G_{v}=G$;

4. $\left(X(T)^{\vee}\right)^{G_{v}}$ est de rang 1 ; 
5. $\operatorname{deg}_{T, L, \mathcal{V}}: T\left(K_{v}\right) \rightarrow\left(X(T)^{\vee}\right)^{G_{v}}$ est surjective.

Alors il existe un $G$-éventail $\Sigma$ projectif et lisse de $X(T)^{\vee}$ tel que $v$ ne vérifie pas l'hypothèse 4.8 .

Démonstration: Soit $m$ un générateur de $\left(X(T)^{\vee}\right)^{G_{v}}$. D'après [CTHaSk], on peut construire un $G$-éventail $\Sigma$ projectif et lisse dont $\mathbf{R}_{\geqslant 0} m$ est l'un des rayons. Comme $\mathbf{R}_{\geqslant 0} m \cap X(T)^{\vee}=m$ et que $m$ est $G$-invariant, l'élément $\varphi$ de $\operatorname{PL}(\Sigma)$ qui envoie $m$ sur 1 et tous les autres rayons sur 0 est dans $\operatorname{PL}(\Sigma)^{G}$. Soit $t$ un élément de $T\left(K_{v}\right)$ vérifiant $\operatorname{deg}_{T, L, \mathcal{V}}(t)=m$. Alors on a $\varphi\left(\operatorname{deg}_{T, L, \mathcal{V}}(t)\right)=\varphi(m)=1$. Ainsi

$$
f_{v}\langle\varphi, t\rangle_{\Sigma, v}=\frac{1}{e_{v}} \varphi\left(\operatorname{deg}_{T, L, \mathcal{V}}(t)\right)=\frac{1}{e_{v}} .
$$

\subsubsection{Hauteurs globales et fonction zêta des hauteurs}

Pour $t \in T(K)$ et $\varphi \in \operatorname{PL}(\Sigma)_{\mathbf{C}}^{G}$ on pose

$$
H(\varphi, t)=\prod_{v \in \mathcal{P}_{K}} H_{v}(\varphi, t)
$$

Si $\varphi$ est un élément de $P L(\Sigma)^{G}$, d'après le point 4 du lemme 4.6 et la définition $(2.8) H(\varphi,$.$) est la restriction à T(K)$ d'une hauteur d'Arakelov associée au faisceau inversible $\mathcal{O}_{X_{\Sigma}}(-\varphi)$.

On pose alors

$$
\zeta_{H(\varphi, .)}(s)=\sum_{t \in T(K)} H(\varphi, t)^{-s}
$$

pour tout $s \in \mathbf{C}$ tel que la série converge,

Plus généralement, on pose

$$
\zeta_{H}(\varphi)=\sum_{t \in T(K)} H(-\varphi, t)
$$

pour tout $\varphi \in P L(\Sigma)_{\mathbf{C}}^{G}$ tel que la série converge.

\subsection{Mesure et nombre de Tamagawa d'une variété torique}

On note $\varphi_{0}$ l'élément de $P L(\Sigma)^{G}$ correspondant au diviseur $\sum_{\alpha \in \Sigma(1) / G} D_{\alpha}$. D'après la proposition $4.1, \mathcal{O}_{X_{\Sigma}}\left(\varphi_{0}\right)$ est isomorphe au faisceau anticanonique de $X_{\Sigma}$. Ainsi la métrique adélique $\left(\|.\|_{v}^{\varphi_{0}}\right)$ dont provient la hauteur $H\left(\varphi_{0},.\right)$ induit pour tout $v$ une mesure $\omega_{X_{\Sigma}, v}$ sur $X_{\Sigma}\left(K_{v}\right)$. 
La restriction à $T$ de l'inverse de la section rationnelle canonique de $\mathcal{O}_{X_{\Sigma}}\left(\varphi_{0}\right)$ est une $d$-forme différentielle $K$-rationnelle $T$-invariante sur $T$. Cette restriction s'écrit donc $\alpha \Omega_{T}$, où $\alpha \in K^{*}$ et $\Omega_{T}$ est la forme utilisée dans la section 3.9 pour la construction des mesures de Haar $\omega_{T, v}$ sur $T\left(K_{v}\right)$.

D'après le point $4 \mathrm{du}$ lemme 4.6 , on a donc pour tout $v \in \mathcal{P}_{K}$

$$
\left(\left.|| \Omega_{T}(.)^{-1}\right|_{v} ^{\varphi_{0}}\right)^{-1}=|\alpha|_{v} H_{v}\left(\varphi_{0}, .\right) .
$$

En vue du calcul du terme principal de la fonction zêta des hauteurs, il faut comparer les mesures $\omega_{X_{\Sigma}, v}$ et $H_{v}\left(\varphi_{0},.\right) \omega_{T, v}$ sur $T\left(K_{v}\right)$. C'est l'objet de la proposition 3.4.4. de [BaTs1], dont nous rappelons l'énoncé et la démonstration.

\section{Lemme 4.10 (Batyrev, Tschinkel)}

Il existe une famille $\left(\alpha_{v}\right) \in\left(\boldsymbol{R}_{>0}\right)^{\mathcal{P}_{K}}$ avec $\alpha_{v}=1$ pour presque tout $v$ et

$$
\prod_{v \in \mathcal{P}_{K}} \alpha_{v}=1
$$

telle que

$$
\forall v \in \mathcal{P}_{K},\left.\quad \omega_{X_{\Sigma}, v}\right|_{T\left(K_{v}\right)}=\alpha_{v} H_{v}\left(\varphi_{0}, .\right) \omega_{T, v} .
$$

Démonstration : Soient $t \in T\left(K_{v}\right)$ et $U$ un ouvert de Zariski de $T$ contenant $t$ tel qu'il existe un $K$-morphisme étale

$$
f: U \longrightarrow \mathbf{A}_{K}^{d},
$$

vérifiant (en notant $\left(y_{1}, \ldots, y_{d}\right)$ les coordonnées sur $\left.\mathbf{A}_{K}^{d}\right)$

$$
f^{*}\left(d y_{1} \wedge \cdots \wedge d y_{d}\right)=\beta^{-1} \Omega_{U},
$$

où $\beta$ est une fonction régulière inversible sur $U$. Ce morphisme induit pour un ouvert analytique $W$ de $U\left(K_{v}\right)$ contenant $t$ et assez petit un isomorphisme analytique

$$
f: W \stackrel{\sim}{\rightarrow} f(W) \subset K_{v}^{d} .
$$

Sur $W$, la mesure $\omega_{T, v}$ s'exprime alors comme

$$
\omega_{T, v}=|\beta|_{v}\left(f^{-1}\right)_{*}\left(d y_{1} \ldots d y_{d}\right) .
$$

Par ailleurs, sur $W$ on a

$$
\omega_{X_{\Sigma}, v}=|\beta|_{v}\left(f^{-1}\right)_{*}\left(\left.|| \Omega_{T}\left(f^{-1}(y)\right)^{-1}\right|_{v} ^{\varphi_{0}} d y_{1} \ldots d y_{d}\right)
$$

soit d'après (4.1)

$$
\omega_{X_{\Sigma}, v}=|\beta|_{v}\left(f^{-1}\right)_{*}\left(|\alpha|_{v} H_{v}\left(\varphi_{0}, f^{-1}(y)\right) d y_{1} \ldots d y_{d}\right)
$$


d'où le résultat.

Rappelons (cf. section 2.3) que le nombre de Tamagawa de $X_{\Sigma}$ est alors par définition

$$
\gamma_{H}\left(X_{\Sigma}\right)=\omega_{X_{\Sigma}}\left(\overline{X_{\Sigma}(K)}\right)
$$

où

$$
\omega_{X_{\Sigma}}=c_{K, \operatorname{dim}\left(X_{\Sigma}\right)} \ell\left(\operatorname{Pic}\left(X_{\Sigma, L}\right)\right) \prod_{v \in \mathcal{P}_{K}} L_{v}\left(1, \operatorname{Pic}\left(X_{\Sigma, L}\right)\right)^{-1} \omega_{X_{\Sigma}, v} .
$$

\section{Lemme 4.11 (Batyrev, Tschinkel)}

On a

$$
\int_{\overline{T(K)} \cap T\left(\boldsymbol{A}_{K}\right)} \omega_{X_{\Sigma}}=\int_{\frac{X_{\Sigma}(K)}{X_{\Sigma}}} \omega_{X_{\Sigma}}
$$

Démonstration : C'est la proposition 3.4.5 de [BaTs1], énoncée uniquement dans le cas arithmétique, mais la preuve marche aussi dans le cas fonctionnel. Nous la rappelons.

Soit ${\overline{X_{\Sigma}(K)}}^{S}$ l'adhérence de l'image de $X_{\Sigma}(K)$ dans $\prod_{v \in S} X_{\Sigma}\left(K_{x}\right)$. Rappelons que nous avions noté $\overline{T(K)}^{S}$ l'adhérence de l'image de $T(K)$ dans $\prod_{v \in S} T\left(K_{v}\right)$. Comme $\prod_{v \in S} T\left(K_{v}\right)$ est ouvert dans $\prod_{v \in S} X_{\Sigma}\left(K_{v}\right)$, on a

$$
\overline{X_{\Sigma}(K)} S \cap \prod_{v \in S} T\left(K_{v}\right)=\overline{\left(\prod_{v \in S} T\left(K_{v}\right)\right) \cap X_{\Sigma}(K)}=\overline{T(K)} S
$$

On a évidemment

$$
\overline{X_{\Sigma}(K)} \subset \overline{X_{\Sigma}(K)} S \times \prod_{v \notin S} X_{\Sigma}\left(K_{v}\right) .
$$

Montrons l'inclusion inverse. Soit $W_{S}$ un ouvert de $\overline{X_{\Sigma}(K)} S, T$ un sousensemble fini de $\mathcal{P}_{K} \backslash S$ et pour $v \in T, U_{v}$ un ouvert de $X_{\Sigma}\left(K_{v}\right)$. Il suffit de montrer que l'ouvert de $\overline{X_{\Sigma}(K)} S \times \prod_{v \notin S} X_{\Sigma}\left(K_{v}\right)$ défini par

$$
W \stackrel{\text { déf }}{=} W_{S} \times \prod_{v \notin S \cup T} X_{\Sigma}\left(K_{v}\right) \times \prod_{v \in T} U_{v}
$$

rencontre $X_{\Sigma}(K)$. 
Or, pour tout $v$, d'après [PlRa, Lemma 3.2], $T\left(K_{v}\right)$ est dense dans $X_{\Sigma}\left(K_{v}\right)$. On en déduit que pour $v \in T, U_{v}$ rencontre $T\left(K_{v}\right)$. et que $W_{S}$ rencontre $\prod_{v \in S} T\left(K_{v}\right)$. Comme $W_{S}$ est inclus dans $\overline{X_{\Sigma}(K)}{ }^{S}, W_{S}$ rencontre en fait

$$
{\overline{X_{\Sigma}(K)}}^{S} \cap \prod_{v \in S} T\left(K_{v}\right)=\overline{T(K)}^{S} .
$$

Ainsi $W$ rencontre $\overline{T(K)}{ }^{S} \times \prod_{v \notin S} T\left(K_{v}\right)$. Comme ce dernier ensemble est inclus dans ${\overline{X_{\Sigma}(K)}}^{S} \times \prod_{v \notin S} X_{\Sigma}\left(K_{v}\right), W \cap \overline{T(K)}^{S} \times \prod_{v \notin S} T\left(K_{v}\right)$ est ouvert dans $\overline{T(K)}^{S} \times \prod_{v \notin S} T\left(K_{v}\right)$. D'après $(3.10)$, ce dernier ensemble coïncide avec $\overline{T(K)}$. On en déduit que $W$ rencontre $T(K)$.

On a donc montré

$$
\overline{X_{\Sigma}(K)}=\overline{X_{\Sigma}(K)} S \times \prod_{v \notin S} X_{\Sigma}\left(K_{v}\right) .
$$

Ainsi

$$
\frac{\int}{X_{\Sigma}(K)} \omega_{X_{\Sigma}}=\int_{X_{\Sigma}(K)} \otimes_{v \in S} \omega_{v, X_{\Sigma}} \times \prod_{v \notin S} \omega_{v, X_{\Sigma}}\left(X_{\Sigma}\left(K_{v}\right)\right) .
$$

Comme $X_{\Sigma} \backslash T$ est un fermé algébrique propre de $X_{\Sigma}$, l'ensemble $\left(X_{\Sigma} \backslash\right.$ $T)\left(K_{v}\right)$ est de mesure nulle pour $\omega_{X_{\Sigma}, v}$ d'après [Bki2, 10.1.3 Exemple b) et 10.1.6]. On a donc

$$
\int_{X_{\Sigma}(K)} \omega_{X_{\Sigma}}=\int_{X_{\Sigma}(K)} \otimes_{v \in S} \omega_{v, X_{\Sigma}} \times \prod_{v \notin S} \omega_{v, X_{\Sigma}}\left(T\left(K_{v}\right)\right) .
$$

Par ailleurs on a

$$
\int_{\overline{T(K)} \cap T\left(\mathbf{A}_{K}\right)} \omega_{X_{\Sigma}}=\int_{\frac{T(K)}{S}} \otimes_{v \in S} \omega_{v, X_{\Sigma}} \times \prod_{v \notin S} \omega_{v, X_{\Sigma}}\left(T\left(K_{v}\right)\right)
$$

et il suffit pour conclure de montrer que ${\overline{X_{\Sigma}(K)}}^{S} \backslash \overline{T(K)}^{S}$ est de mesure nulle pour $\otimes_{v \in S} \omega_{v, X_{\Sigma}}$.

$$
\begin{aligned}
& \text { Comme } \overline{X_{\Sigma}(K)} S \cap \prod_{v \in S} T\left(K_{v}\right)=\overline{T(K)}{ }^{S} \text {, on a } \\
&\left(\overline{X_{\Sigma}(K)} S \backslash \overline{T(K)} S\right) \subset\left(\prod_{v \in S} X_{\Sigma}\left(K_{v}\right) \backslash \prod_{v \in S} T\left(K_{v}\right)\right)
\end{aligned}
$$


Pour tout $v$, on a $\omega_{X_{\Sigma}, v}\left(X_{\Sigma}\left(K_{v}\right) \backslash T\left(K_{v}\right)\right)=0$, donc $\prod_{v \in S} X_{\Sigma}\left(K_{v}\right) \backslash \prod_{v \in S} T\left(K_{v}\right)$ est de mesure nulle pour $\otimes_{v \in S} \omega_{X_{\Sigma}, v}$, d'où le résultat d'après (4.21).

\subsection{Le résultat}

Nous sommes à présent en mesure d'énoncer le résultat obtenu par Batyrev et Tschinkel dans [BaTs2], ainsi que notre résultat qui en est un analogue dans le cas fonctionnel.

\section{Théorème 4.12 (Batyrev, Tschinkel)}

Soit $K$ un corps de nombres, $L / K$ une extension finie galoisienne de groupe $G$, et $\Sigma$ un $G$-éventail projectif et lisse. Soit $X_{\Sigma}$ la variété torique projective et lisse, définie sur $K$, qui lui est associée. C'est une compactification d'un tore algébrique $T$.

Alors la série $\zeta_{H}\left(s \varphi_{0}\right)$ converge absolument pour $s \in \mathcal{T}\left(\boldsymbol{R}_{>1}\right)$ et, pour un certain $\varepsilon>0$, la fonction

$$
f: s \longmapsto(s-1)^{\operatorname{rg}\left(\operatorname{Pic}\left(X_{\Sigma}\right)\right)} \zeta_{H}\left(s \varphi_{0}\right)
$$

se prolonge en une fonction holomorphe sur le domaine $\mathcal{T}\left(\boldsymbol{R}_{>1-\varepsilon}\right)$, vérifiant

$$
f(1)=\alpha^{*}\left(X_{\Sigma}\right)\left[H^{1}\left(G, \operatorname{Pic}\left(X_{\Sigma, L}\right)\right)\right] \gamma_{H}\left(X_{\Sigma}\right)
$$

Nous obtenons pour notre part le théorème suivant.

\section{Théorème 4.13}

Soit $K$ un corps de fonctions, $L / K$ une extension finie galoisienne de groupe $G$, et $\Sigma$ un $G$-éventail projectif et lisse. Soit $X_{\Sigma}$ la variété torique projective et lisse, définie sur $K$, qui lui est associée. C'est une compactification d'un tore algébrique $T$.

Alors la série $\zeta_{H}\left(s \varphi_{0}\right)$ converge absolument pour $s \in \mathcal{T}\left(\boldsymbol{R}_{>1}\right)$ et pour un certain $\varepsilon>0$ se prolonge en une fonction méromorphe sur $\mathcal{T}\left(\boldsymbol{R}_{>1-\varepsilon}\right)$. Ce prolongement a un pôle d'ordre le rang du groupe de Picard de $X_{\Sigma}$ en $s=1$, et on a

$$
\lim _{s \rightarrow 1}(s-1)^{\operatorname{rg}\left(\operatorname{Pic}\left(X_{\Sigma}\right)\right)} \zeta_{H}\left(s \phi_{0}\right)=\alpha^{*}\left(X_{\Sigma}\right)\left[H^{1}\left(G, \operatorname{Pic}\left(X_{\Sigma, L}\right)\right)\right] \gamma_{H}\left(X_{\Sigma}\right) .
$$

Remarques 4.14:

1. Notre preuve, comme déjà indiqué, est fortement inspirée des preuves de [BaTs1] et [BaTs2]. 
2. Dans le cas arithmétique comme dans le cas fonctionnel, l'ensemble $X_{\Sigma}(K)$ est bien Zariski dense dans $X_{\Sigma}$ d'après [BoSp, Corollary 7.12].

3. Contrairement au cas arithmétique, on ne peut espérer dans le cas fonctionnel prolonger la fonction $s \mapsto(s-1)^{\operatorname{rg}\left(\operatorname{Pic}\left(X_{\Sigma}\right)\right)} \zeta_{H}\left(s \phi_{0}\right)$ en une fonction holomorphe sur un domaine du type $\mathcal{T}\left(\mathbf{R}_{>1-\varepsilon}\right)$. En effet dans ce cas la fonction zêta des hauteurs a d'autres pôles sur la droite $\{\Re(s)=1\}$, ne serait-ce que ceux dû au fait qu'elle admet des périodes imaginaires pures. Par exemple, au moins dans le cas où la variété $X_{\Sigma}$ vérifie l'hypothèse 4.8 pour toute place $v$, la fonction $\zeta_{H}\left(s \phi_{0}\right)$ est $\frac{2 i \pi}{\log (q)}$-périodique.

\subsection{Stratégie de Batyrev et Tschinkel}

Une des idées essentielles de Batyrev et Tschinkel pour étudier la fonction zêta des hauteurs des variétés toriques est d'appliquer la formule de Poisson pour obtenir une représentation intégrale de $\zeta_{H}(\varphi)$. Avant de préciser ce qui précède, rappelons quelques faits élémentaires d'analyse harmonique abstraite.

\subsubsection{Un peu d'analyse harmonique}

On note $\boldsymbol{U}$ le groupe des nombres complexes de module 1. Soit $\mathcal{G}$ un groupe abélien localement compact. Son dual topologique est le groupe topologique, noté $\mathcal{G}^{*}$, formé de l'ensemble des morphismes continus de $\mathcal{G}$ dans $\boldsymbol{U}$. C'est encore un groupe abélien localement compact.

Exemples 4.15: Soit $M$ un Z-module libre de rang fini.

1. Le morphisme qui à $m \in M_{\mathbf{R}}^{\vee}$ associe le caractère

$$
m \mapsto \exp \left(i\left\langle m^{\vee}, m\right\rangle\right) \text {. }
$$

est un isomorphisme du groupe topologique $M_{\mathbf{R}}^{\vee}$ sur le groupe topologique $M_{\mathbf{R}}^{*}$. Par la suite, on identifiera toujours $M_{\mathbf{R}}^{*}$ à $M_{\mathbf{R}}^{\vee}$ au moyen de cet isomorphisme.

2. Le morphisme qui à $m^{\vee} \otimes z \in M_{\boldsymbol{U}}^{\vee}$ associe

$$
m \mapsto z^{\left\langle m^{\vee}, m\right\rangle}
$$

est un isomorphisme du groupe topologique $M_{U}^{\vee}$ sur le dual topologique de $M$. Par la suite, on identifiera toujours $M^{*}$ à $M_{U}^{\vee}$ au moyen de cet isomorphisme. 
Soit $\mathcal{G}$ un groupe abélien localement compact muni d'une mesure de Haar $d g$. Soit $F: \mathcal{G} \rightarrow \mathbf{C}$ une fonction de classe $\mathbf{L}^{1}$. Sa transformée de Fourier par rapport à $d g$ est la fonction $\mathcal{F} F: \mathcal{G}^{*} \rightarrow \mathbf{C}$ définie par

$$
\forall \chi \in \mathcal{G}^{*}, \quad \mathcal{F} F(\chi)=\int_{\mathcal{G}} F(g) \chi(g) d g .
$$

Il existe alors (cf. [Bki1, Définition 4, p.118 et Théorème 3, p. 123]) une unique mesure de Haar $d g^{*}$ sur $\mathcal{G}^{*}$ vérifiant la formule d'inversion de Fourier, c'est-à-dire la propriété suivante $:$ soit $F: \mathcal{G} \rightarrow \mathbf{C}$ une fonction de classe $\mathbf{L}^{1}$ telle que $\mathcal{F} F$ est de classe $\mathbf{L}^{1}$ sur $\mathcal{G}^{*}$; alors, pour presque tout $g$ de $\mathcal{G}$, on a la formule

$$
F(g)=\int_{\mathcal{G}^{*}} \overline{\chi(g)}(\mathcal{F} F)(\chi) d g^{*}(\chi) .
$$

La mesure de Haar $d g^{*}$ sera appelée mesure duale de la mesure de Haar $d g$. Moyennant l'identification canonique de $\left(\mathcal{G}^{*}\right)^{*}$ à $\mathcal{G}$, on a $\left(d g^{*}\right)^{*}=d g$.

Le lemme suivant donne deux exemples standards de mesure duale.

\section{Lemme 4.16}

Soit $\mathcal{G}$ un groupe topologique abélien localement compact, et $d g$ une mesure de Haar sur $\mathcal{G}$.

1. On suppose que $\mathcal{G}$ est compact, de sorte que $\mathcal{G}^{*}$ est discret. Alors $d g^{*}$ est la mesure de Haar sur $\mathcal{G}^{*}$ pour laquelle chaque point a pour masse $\frac{1}{\int_{\mathcal{G}} d g}$.

2. On suppose que $\mathcal{G}=N_{\boldsymbol{R}}$ où $N$ est un $\mathbf{Z}$-module libre de rang fini et que $d g$ est la mesure de Lebesgue sur $N_{\boldsymbol{R}}$ normalisée par le réseau $N$. Alors $d g^{*}$ est la mesure de Lebesgue sur $N_{\boldsymbol{R}}^{\vee}$ normalisée par le réseau $N^{\vee}$, divisée par $(2 \pi)^{\operatorname{rg}(N)}$.

Démonstration: On applique (4.4) en prenant pour $F$ l'indicatrice de $G$ dans le premier cas, et l'application $\left(x_{1}, \ldots, x_{n}\right) \mapsto \exp \left(-\pi\left(x_{1}^{2}+\cdots+x_{n}^{2}\right)\right)$ dans le second cas.

On peut à présent rappeler un énoncé de la formule de Poisson (cf. [Bki1, Proposition 8, p. 127]).

\section{Théorème 4.17 (Formule de Poisson)}

Soit $\mathcal{G}$ un groupe abélien localement compact muni d'une mesure de Haar $d g$, et $\mathcal{H}$ un sous-groupe fermé de $\mathcal{G}$ muni d'une mesure de Haar dh. Soit dx la mesure de Haar sur le quotient $\mathcal{G} / \mathcal{H}$ normalisée par la relation $d g=d x d h$. 
On munit le groupe $(\mathcal{G} / \mathcal{H})^{*}$ de la mesure de Haar $d x^{*}$ duale de la mesure $d x$, et on identifie ce groupe au sous-groupe de $\mathcal{G}^{*}$ constitué des caractères de $\mathcal{G}$ triviaux sur $\mathcal{H}$.

Soit $F: \mathcal{G} \rightarrow \boldsymbol{C}$ une fonction de classe $\boldsymbol{L}^{1}$ et $\mathcal{F} F$ sa transformée de Fourier par rapport à $d g$. On suppose que $\mathcal{F} F$ est $\boldsymbol{L}^{1} \operatorname{sur}(\mathcal{G} / \mathcal{H})^{*}$.

Alors, pour presque tout $g$ de $\mathcal{G}$, la propriété suivante est vérifiée : la fonction $h \mapsto F(g h)$ est $\boldsymbol{L}^{1}$ sur $\mathcal{H}$ et on a la formule

$$
\int_{\mathcal{H}} F(g h) d h=\int_{(\mathcal{G} / \mathcal{H})^{*}} \overline{\chi(g)} \mathcal{F} F(\chi) d x^{*}(\chi) .
$$

\section{Corollaire 4.18}

On conserve les notations et hypothèses du théorème 4.17. On se donne en outre un sous-groupe compact $\mathcal{K}$ de $\mathcal{G}$, de volume non nul, et on suppose que la fonction $F$ est $\mathcal{K}$-invariante. Alors la fonction $F$ est $\boldsymbol{L}^{1}$ sur $\mathcal{H}$ et on a la formule

$$
\int_{\mathcal{H}} F(h) d h=\int_{(\mathcal{G} / \mathcal{K} . \mathcal{H})^{*}} \mathcal{F} F(\chi) d x^{*}(\chi) .
$$

Remarque 4.19 : Comme $\mathcal{K}$ est compact, $(\mathcal{G} / \mathcal{K} . \mathcal{H})^{*}$ est un sous-groupe ouvert de $(\mathcal{G} / \mathcal{H})^{*}$, et la restriction de $d x^{*}$ à $(\mathcal{G} / \mathcal{K} . \mathcal{H})^{*}$ est une mesure de Haar sur $(\mathcal{G} / \mathcal{K} . \mathcal{H})^{*}$.

Comme $F$ est $\mathcal{K}$-invariante, $\mathcal{F} F(\chi)$ est nulle si $\chi$ n'est pas trivial sur $\mathcal{K}$. Ainsi l'hypothèse que $\mathcal{F} F$ est $\mathbf{L}^{1}$ sur $(\mathcal{G} / \mathcal{H})^{*}$ équivaut à l'hypothèse que $\mathcal{F} F$ est $\mathbf{L}^{1} \operatorname{sur}(\mathcal{G} / \mathcal{K} . \mathcal{H})^{*}$

Démonstration : D'après le théorème 4.17 , comme $\mathcal{K}$ est de volume non nul, il existe un élément $k$ de $\mathcal{K}$ tel que $h \mapsto F(k . h)$ est de classe $\mathbf{L}^{1}$ sur $\mathcal{H}$ et tel qu'on ait la formule

$$
\int_{\mathcal{H}} F(k . h) d h=\int_{(\mathcal{G} / \mathcal{H})^{*}} \overline{\chi(k)}(\mathcal{F F})(\chi) d x^{*}(\chi) .
$$

Comme $F$ est $\mathcal{K}$-invariante, $\mathcal{F} F(\chi)$ est nulle si $\chi$ n'est pas trivial sur $\mathcal{K}$. Le corollaire s'en déduit aussitôt.

\subsubsection{Application à la fonction zêta des hauteurs}

Pour appliquer ce qui précède à l'étude de la fonction zêta des hauteurs, on commence par étendre la hauteur en une fonction continue sur $T\left(\mathbf{A}_{K}\right)$ en posant pour tout $\left(t_{v}\right) \in T\left(\mathbf{A}_{K}\right)$ et tout $\varphi$ de $\operatorname{PL}(\Sigma)_{\mathbf{C}}^{G}$

$$
H\left(\varphi,\left(t_{v}\right)\right)=\prod_{v \in \mathcal{P}_{K}} H_{v}\left(\varphi, t_{v}\right) .
$$


Ainsi $H(\varphi,$.$) est invariante sous l'action de \mathbf{K}(T)$ (ceci sera utile pour annuler beaucoup de transformées de Fourier).

Par la suite, pour tout $\varphi$ de $\operatorname{PL}(\Sigma)_{\mathbf{C}}^{G}$, nous noterons pour alléger l'écriture $H(\varphi)$ la fonction $H(\varphi,$.$) .$

On va appliquer le corollaire 4.18 à $\mathcal{G}=T\left(\mathbf{A}_{K}\right)$ muni de la mesure de Haar $\omega_{T}$ définie à la section $3.9, \mathcal{H}=T(K)$, identifié à un sous-groupe discret de $T\left(\mathbf{A}_{K}\right)$ et muni de la mesure discrète, et $\mathcal{K}=\mathbf{K}(T)$ (notons qu'on a bien $\int \omega_{T} \neq 0$, cf. la section 3.9). $\mathbf{K}(T)$

La mesure de Haar sur $\left(T\left(\mathbf{A}_{K}\right) / T(K)\right)^{*}$ duale de la mesure quotient sur $T\left(\mathbf{A}_{K}\right) / T(K)$, sera notée $d \chi$.

Compte tenu du fait que $H(-\varphi)$ est $\mathbf{K}(T)$-invariante pour tout élément $\varphi$ de $\operatorname{PL}(\Sigma)^{G}$, on déduit immédiatement du corollaire 4.18 le lemme suivant.

\section{Lemme 4.20}

Soit $\varphi$ un élément de $\mathrm{PL}(\Sigma)^{G}$ tel que $H(-\varphi)$ est $\boldsymbol{L}^{1}$ sur $T\left(\boldsymbol{A}_{K}\right)$. Soit $\mathcal{F} H(-\varphi)$ la transformée de Fourier de $H(-\varphi)$ par rapport à la mesure $\omega_{T}$. Supposons en outre que $\mathcal{F} H(-\varphi)$ est $\boldsymbol{L}^{1} \operatorname{sur}\left(T\left(\boldsymbol{A}_{K}\right) / \boldsymbol{K}(T) \cdot T(K)\right)^{*}$.

Alors $H(-\varphi)$ est $\boldsymbol{L}^{1}$ sur $T(K)$ et on a la formule

$$
\left.\sum_{t \in T(K)} H(-\varphi, t)=\int_{\left(T\left(\boldsymbol{A}_{K}\right) / \boldsymbol{K}(T)\right.} \mathcal{F} H(K)\right)^{*}(-\varphi)(\chi) d \chi .
$$

Remarque 4.21: Si $H(-\varphi)$ est intégrable, la fonction $\mathcal{F} H(-\varphi)$ est continue sur $T\left(\mathbf{A}_{K}\right)^{*}$, et donc sa restriction à $\left(T\left(\mathbf{A}_{K}\right) / T(K)\right)^{*}$ est intégrable sur tout compact.

Or, dans le cas fonctionnel, $\left(T\left(\mathbf{A}_{K}\right) / \mathbf{K}(T) \cdot T(K)\right)^{*}$ est compact. Ainsi dans ce cas l'intégrabilité de $H(-\varphi)$ entraîne automatiquement l'intégrabilité de $\mathcal{F} H(-\varphi)$.

Le but de la partie suivante est de montrer, via un calcul explicite des transformées de Fourier locales en presque toutes les places et des estimées ad hoc aux places restantes, que l'expression sous l'intégrale dans la formule (4.9) est une fonction méromorphe de $\varphi$ dont on contrôle les pôles.

Les lemmes techniques rappelés dans la partie 6 pour le cas arithmétique et développés dans la partie 7 dans le cas fonctionnel permettront ensuite de montrer que l'intégrale elle-même est une fonction méromorphe dont on contrôle le comportement analytique. 


\section{Calcul des transformées de Fourier et expres- sion intégrale de la fonction zêta des hauteurs}

\subsection{Caractères du groupe des idèles}

Soit $E$ un corps global.

Si $v$ est une place non archimédienne de $E$, tout caractère de $\mathbf{G}_{m}\left(K_{v}\right)$ trivial sur $\mathbf{G}_{m}\left(\mathcal{O}_{v}\right)$ est de la forme

$$
x \mapsto z^{v(x)}
$$

où $z$ est un élément de $\boldsymbol{U}$.

Si $v$ est une place archimédienne de $E$, tout caractère de $\mathbf{G}_{m}\left(K_{v}\right)$ trivial sur $\mathbf{G}_{m}\left(\mathcal{O}_{v}\right)$ est de la forme

$$
x \mapsto e^{i t \log |x|_{v}}
$$

où $t$ est un élément de $\mathbf{R}$.

Soit à présent $\chi$ un caractère de $\mathbf{G}_{m}\left(\mathbf{A}_{E}\right)$. Pour $v \in \mathcal{P}_{E}$ on note $\chi_{v}$ le caractère induit sur $\mathbf{G}_{m}\left(E_{v}\right)$ via l'injection naturelle de $\mathbf{G}_{m}\left(E_{v}\right)$ dans $\mathbf{G}_{m}\left(\mathbf{A}_{E}\right)$. Si $\chi$ est trivial sur $\mathbf{K}\left(\mathbf{G}_{m}\right)$, il s'écrit

$$
\chi:\left(x_{v}\right) \longmapsto \prod_{v \in \mathcal{P}_{E, f}} z_{v}^{v\left(x_{v}\right)} \prod_{v \in \mathcal{P}_{E, \infty}} e^{i t_{v} \log \left|x_{v}\right|_{v}}
$$

$\operatorname{avec}\left(z_{v}\right) \in \boldsymbol{U}^{\mathcal{P}_{E, f}}$ et $\left(t_{v}\right) \in \mathbf{R}^{\mathcal{P}_{E, \infty}}$. Pour tout $v \in \mathcal{P}_{E, f}$ on a donc $z_{v}=\chi_{v}\left(\pi_{v}\right)$.

Si $\chi$ est de plus trivial sur $\mathbf{G}_{m}\left(\mathbf{A}_{E}\right)^{1}$, il s'écrit $\chi^{\prime} \circ \operatorname{deg}_{E}$, où $\chi^{\prime}$ est un caractère de $\mathbf{G}_{m}\left(\mathbf{A}_{E}\right) / \mathbf{G}_{m}\left(\mathbf{A}_{E}\right)^{1}$. Dans le cas arithmétique, ce dernier groupe est isomorphe à $\mathbf{R}$ et il existe donc un $y \in \mathbf{R}$ tel que $\chi$ s'écrit

$$
x \longmapsto e^{i y \operatorname{deg}_{E}(x)} .
$$

Dans le cas fonctionnel, $\mathbf{G}_{m}\left(\mathbf{A}_{E}\right) / \mathbf{G}_{m}\left(\mathbf{A}_{E}\right)^{1}$ est isomorphe à $\mathbf{Z}$ et il existe donc un $z \in \boldsymbol{U}$ tel que $\chi$ s'écrit

$$
x \longmapsto z^{\operatorname{deg}_{E}(x)} .
$$

Revenons plus généralement à un caractère de $\mathbf{G}_{m}\left(\mathbf{A}_{E}\right)$ trivial sur $\mathbf{K}\left(\mathbf{G}_{m}\right)$. On définit la fonction $L$ associée :

$$
L_{E}(\chi, s)=\prod_{v \in \mathcal{P}_{E, f}} \frac{1}{1-\chi_{v}\left(\pi_{v}\right) q_{v}^{-s}} .
$$

Ce produit eulérien converge absolument pour $\Re(s)>1$.

D'après [We1, VII, $\S 7$, Thm 6], on a 


\section{Lemme 5.1}

Si $\chi$ n'est pas trivial sur $\boldsymbol{G}_{m}\left(\boldsymbol{A}_{E}\right)^{1}$, la fonction $s \mapsto L_{E}(s, \chi)$ est holomorphe sur $\boldsymbol{C}$. Dans le cas fonctionnel, elle s'exprime comme un polynôme en $q_{E}^{-s}$.

Dans le cas fonctionnel on notera $\mathcal{L}_{E}(\chi$, . ) le polynôme vérifiant

$$
\mathcal{L}_{E}\left(\chi, q_{E}^{-s}\right)=L_{E}(\chi, s) .
$$

\subsection{Caractères de $T\left(\mathbf{A}_{K}\right)$}

Soit $T$ un tore algébrique défini sur un corps global $K$. Nous reprenons les notations de la partie 4.1.2, le corps de base étant bien sûr le corps global $K$. Dans le cas fonctionnel, le corps des constantes de $K$ est désormais supposé de cardinal $q$.

Rappelons en particulier qu'on a une suite exacte de $G$-modules

$$
0 \longrightarrow X(T) \stackrel{\gamma}{\longrightarrow} P_{\Sigma} \longrightarrow \operatorname{Pic}\left(X_{\Sigma, L}\right) \longrightarrow 0
$$

et que $\operatorname{Pic}\left(X_{\Sigma, L}\right)$ est un $G$-module flasque.

Pour $\chi \in T\left(\mathbf{A}_{K}\right)^{*}$, on note $\chi_{\alpha}$ le caractère $\chi \circ \gamma_{\mathbf{A}_{K}} \circ i_{\alpha, \mathbf{A}_{K}}$, de sorte que $\chi_{\alpha}$ est un élément de $\mathbf{G}_{m}\left(\mathbf{A}_{K_{\alpha}}\right)^{*}$.

Le but de ce qui suit est de décrire le morphisme de caractères

$$
\left(T\left(\mathbf{A}_{K}\right) / T\left(\mathbf{A}_{K}\right)^{1}\right)^{*} \longrightarrow\left(T_{P_{\Sigma}}\left(\mathbf{A}_{K}\right) / T_{P_{\Sigma}}\left(\mathbf{A}_{K}\right)^{1}\right)^{*}
$$

induit par la composition avec $\gamma_{\mathbf{A}_{K}}$, à l'aide des morphisme de caractères

$$
\begin{aligned}
\left(T\left(\mathbf{A}_{K}\right) / T\left(\mathbf{A}_{K}\right)^{1}\right)^{*} & \longrightarrow\left(\mathbf{G}_{m}\left(\mathbf{A}_{K_{\alpha}}\right) / \mathbf{G}_{m}\left(\mathbf{A}_{K_{\alpha}}\right)^{1}\right)^{*} . \\
\chi & \longmapsto \chi_{\alpha}
\end{aligned}
$$

\subsubsection{Cas arithmétique}

Commenc̀ ;ons par décrire les caractères de $T\left(\mathbf{A}_{K}\right)$ triviaux sur $T\left(\mathbf{A}_{K}\right)^{1}$. On a un morphisme surjectif

$$
\operatorname{deg}_{T}: T\left(\mathbf{A}_{K}\right) \longrightarrow \operatorname{Hom}\left(X(T)^{G}, \mathbf{R}\right)
$$

de noyau $T\left(\mathbf{A}_{K}\right)^{1}$, qui induit donc un isomorphisme

$$
\operatorname{deg}_{T}: T\left(\mathbf{A}_{K}\right) / T\left(\mathbf{A}_{K}\right)^{1} \stackrel{\sim}{\longrightarrow} \operatorname{Hom}\left(X(T)^{G}, \mathbf{R}\right) .
$$

Pour tout $y \in X(T)_{\mathbf{R}}^{G}$, on note $\chi_{y}$ l'élément de $\left(T\left(\mathbf{A}_{K}\right) / T\left(\mathbf{A}_{K}\right)^{1}\right)^{*}$ qui lui correspond via l'isomorphisme dual de (5.5) (cf. la convention 4.15). On a donc

$$
\forall t \in T\left(\mathbf{A}_{K}\right), \quad \chi_{y}(t)=\exp \left(i\left\langle y, \operatorname{deg}_{T}(t)\right\rangle\right) .
$$


En particulier, si $v$ est une place de $K$, on a

$$
\begin{aligned}
\forall t \in T\left(K_{v}\right), \quad\left(\chi_{y}\right)_{v}(t) & =\exp \left(i\left\langle y, \operatorname{deg}_{T} \circ i_{T, v}(t)\right\rangle\right) \\
& =\exp \left(i \log \left(q_{v}\right)\left\langle y, \operatorname{deg}_{T, v}(t)\right\rangle\right) .
\end{aligned}
$$

On notera aussi $y_{\alpha}$ le réel $\left\langle\rho_{\alpha}, y\right\rangle$.

\section{Lemme 5.2}

Soit $y \in X(T)_{\boldsymbol{R}}^{G}$. Soit $\alpha \in \Sigma(1) / G$. Alors pour $x \in \boldsymbol{G}_{m}\left(\boldsymbol{A}_{K_{\alpha}}\right)$ on a

$$
\left(\chi_{y}\right)_{\alpha}(x)=\exp \left(i\left\langle\rho_{\alpha}, y\right\rangle \operatorname{deg}_{K_{\alpha}}(x)\right)
$$

Démonstration : On a

$$
\begin{aligned}
\left(\chi_{y}\right)_{\alpha}(x) & =\chi_{y}\left(\gamma_{\mathbf{A}_{K}} \circ i_{\alpha, \mathbf{A}_{K}}(x)\right) \\
& =\exp \left(i\left\langle y, \operatorname{deg}_{T} \circ \gamma_{\mathbf{A}_{K}} \circ i_{\alpha, \mathbf{A}_{K}}(x)\right\rangle\right) \\
& =\exp \left(i\left\langle y, \gamma_{\mathbf{R}}^{\vee} \circ i_{\alpha, \mathbf{R}}^{\vee}\left[\operatorname{deg}_{\operatorname{Res}_{K_{\alpha} / K} \mathbf{G}_{m}}(x)\right]\right\rangle\right) \\
& =\exp \left(i\left\langle y, \gamma_{\mathbf{R}}^{\vee} \circ i_{\alpha, \mathbf{R}}^{\vee}\left[\operatorname{deg}_{K_{\alpha}}(x)\right]\right\rangle\right) \\
& =\exp \left(i\left\langle y, \gamma_{\mathbf{R}}^{\vee}\left[\operatorname{deg}_{K_{\alpha}}(x) D_{\alpha}^{\vee}\right]\right\rangle\right) \\
& =\exp \left(i \operatorname{deg}_{K_{\alpha}}(x)\left\langle y, \rho_{\alpha}\right\rangle\right) .
\end{aligned}
$$

Le passage de (5.11) à (5.12) découle du lemme 3.7, et le passage de (5.14) à (5.15) vient de (4.15).

\subsubsection{Cas fonctionnel}

Là encore, commenc̀ ;ons par décrire les caractères de $T\left(\mathbf{A}_{K}\right)$ triviaux sur $T\left(\mathbf{A}_{K}\right)^{1}$. On a un morphisme surjectif

$$
\operatorname{deg}_{T}: T\left(\mathbf{A}_{K}\right) \longrightarrow \mathcal{D}_{T}
$$

de noyau $T\left(\mathbf{A}_{K}\right)^{1}$, qui induit donc un isomorphisme

$$
\operatorname{deg}_{T}: T\left(\mathbf{A}_{K}\right) / T\left(\mathbf{A}_{K}\right)^{1} \stackrel{\sim}{\longrightarrow} \mathcal{D}_{T}
$$

et par dualité un isomorphisme entre $\left(T\left(\mathbf{A}_{K}\right) / T\left(\mathbf{A}_{K}\right)^{1}\right)^{*}$ et $\left(\mathcal{D}_{T}^{\vee}\right)_{\boldsymbol{U}}$. Pour tout $\boldsymbol{z} \in X(T)_{\boldsymbol{U}}^{G} \subset\left(\mathcal{D}_{T}^{\vee}\right)_{\boldsymbol{U}}$ on note $\chi_{\boldsymbol{z}}$ le caractère de $\left(T\left(\mathbf{A}_{K}\right) / T\left(\mathbf{A}_{K}\right)^{1}\right)^{*}$ correspondant via cet isomorphisme. On a ainsi

$$
\forall t \in T\left(\mathbf{A}_{K}\right), \quad \chi_{\boldsymbol{z}}(t)=\left\langle\boldsymbol{z}, \operatorname{deg}_{T}(t)\right\rangle .
$$

En particulier, si $v$ est une place de $K$, on a

$$
\forall \boldsymbol{z} \in X(T)_{\boldsymbol{U}}^{G}, \quad \forall x \in T\left(K_{v}\right), \quad\left(\chi_{\boldsymbol{z}}\right)_{v}(x)=\left\langle\boldsymbol{z}, \operatorname{deg}_{T, v}\right\rangle^{f_{v}} .
$$


Nous noterons $d_{\alpha}$ le degré absolu de $K_{\alpha}$. On a donc $\left[\mathbf{F}_{K_{\alpha}}\right]=q^{d_{\alpha}}$. En outre, d'après le lemme 3.15 , on a

$$
\left[\mathcal{C}_{T_{P_{\Sigma}}}\right]=\prod_{\alpha \in \Sigma(1) / G} d_{\alpha} .
$$

Rappelons le début de la suite exacte (4.13)

$$
0 \longrightarrow X(T)^{G} \stackrel{\gamma}{\longrightarrow} P_{\Sigma}^{G} \longrightarrow \operatorname{Pic}\left(X_{\Sigma}\right)
$$

qui montre que $P_{\Sigma}^{G} / X(T)^{G}$ est sans torsion.

Ainsi le morphisme dual de $\gamma$

$$
(\gamma)^{\vee}:\left(P_{\Sigma}^{G}\right)^{\vee} \longrightarrow \operatorname{Hom}\left(X(T)^{G}, \mathbf{Z}\right)
$$

est surjectif. D’après (4.15) ce morphisme envoie $D_{\alpha}^{\vee}$ sur $\rho_{\alpha}$.

On obtient un diagramme

$$
\begin{array}{r}
\mathbf{G}_{m}\left(\mathbf{A}_{K_{\alpha}}\right) \stackrel{i_{\alpha, \mathbf{A}_{K}}}{\longrightarrow} T_{P_{\Sigma}}\left(\mathbf{A}_{K}\right) \stackrel{\gamma_{\mathbf{A}_{K}}}{\longrightarrow} T\left(\mathbf{A}_{K}\right) \\
\operatorname{deg}_{\operatorname{Res}_{K_{\alpha} / K} \mathbf{G} m} \underset{\operatorname{Zeg}_{T} \downarrow}{\operatorname{deg}_{T_{P_{\Sigma}}} \downarrow} \stackrel{i_{\alpha}^{\vee}}{\longrightarrow}\left(P_{\Sigma}^{G}\right)^{\vee} \stackrel{\gamma^{\vee}}{\longrightarrow} \operatorname{Hom}\left(X(T)^{G}, \mathbf{Z}\right)
\end{array}
$$

Le lemme 3.7 montre que ce diagramme est commutatif.

On note $\mathcal{D}_{T}^{0}$ l'image de $\mathcal{D}_{T_{P_{\Sigma}}}$ par $\gamma^{\vee}$ dans $\mathcal{D}_{T}$.

\section{Lemme 5.3}

Soit $\alpha \in \Sigma(1) / G$.

1. $d_{\alpha} \rho_{\alpha}$ est dans $\mathcal{D}_{T}^{0}$.

2. On a

$$
\forall x \in \boldsymbol{G}_{m}\left(\boldsymbol{A}_{K_{\alpha}}\right), \quad \forall \boldsymbol{z} \in X(T)_{\boldsymbol{U}}^{G}, \quad\left(\chi_{\boldsymbol{z}}\right)_{\alpha}(x)=\left\langle\boldsymbol{z}, \operatorname{deg}_{K_{\alpha}}(x) d_{\alpha} \rho_{\alpha}\right\rangle .
$$

Démonstration: Montrons qu'on a, pour tout $x \in \mathbf{G}_{m}\left(\mathbf{A}_{K_{\alpha}}\right)$,

$$
\gamma^{\vee}\left(\operatorname{deg}_{T_{P_{\Sigma}}}\left[i_{\alpha, \mathbf{A}_{K}}(x)\right]\right)=d_{\alpha} \rho_{\alpha} \operatorname{deg}_{K_{\alpha}}(x) .
$$

En effet, on a, par commutativité de (5.23), et le lemme 3.15,

$$
\begin{aligned}
\gamma^{\vee}\left(\operatorname{deg}_{T_{P_{\Sigma}}}\left[i_{\alpha, \mathbf{A}_{K}}(x)\right]\right) & =\gamma^{\vee}\left(i_{\alpha}^{\vee}\left[\operatorname{deg}_{\operatorname{Res}_{K_{\alpha} / K} \mathbf{G}_{m}}(x)\right]\right) \\
& =\gamma^{\vee}\left(i_{\alpha}^{\vee}\left[d_{\alpha} \operatorname{deg}_{K_{\alpha}}(x)\right]\right) \\
& =\operatorname{deg}_{K_{\alpha}}(x) d_{\alpha} \rho_{\alpha} .
\end{aligned}
$$


Le fait que $d_{\alpha} \rho_{\alpha}$ soit dans $\mathcal{D}_{T}^{0}$ découle alors de de (5.25) et de l'existence d'éléments $x$ de $\mathbf{G}_{m}\left(\mathbf{A}_{K_{\alpha}}\right)$ vérifiant $\operatorname{deg}_{K_{\alpha}}(x)=1$.

Toujours par commutativité de (5.23), on a, pour tout $x \in \mathbf{G}_{m}\left(\mathbf{A}_{K_{\alpha}}\right)$,

$\operatorname{deg}_{T}\left(\gamma_{\mathbf{A}_{K}} \circ i_{\alpha, \mathbf{A}_{K}}(x)\right)=\gamma^{\vee}\left(i_{\alpha}\left[d_{\alpha} \operatorname{deg}_{K_{\alpha}}(x)\right]\right)=d_{\alpha} \rho_{\alpha} \operatorname{deg}_{K_{\alpha}}(x)$.

Pour $\boldsymbol{z} \in X(T)_{\boldsymbol{U}}^{G}$, on a alors

$$
\begin{aligned}
\left(\chi_{\boldsymbol{z}}\right)_{\alpha}(x) & =\chi_{\boldsymbol{z}}\left(\gamma_{\mathbf{A}_{K}} \circ i_{\alpha, \mathbf{A}_{K}}(x)\right) \\
& =\left\langle\boldsymbol{z}, \operatorname{deg}_{T}\left(\gamma_{\mathbf{A}_{K}} \circ i_{\alpha, \mathbf{A}_{K}}(x)\right)\right\rangle \\
& =\left\langle\boldsymbol{z}, \operatorname{deg}_{K_{\alpha}}(x) d_{\alpha} \rho_{\alpha}\right\rangle .
\end{aligned}
$$

Remarque 5.4: La commutativité du diagramme (5.23) et la surjectivité du morphisme

$$
\gamma^{\vee}:\left(P_{\Sigma}^{G}\right)^{\vee} \longrightarrow\left(X(T)^{G}\right)^{\vee}
$$

permettent de retrouver le fait que le conoyau de $\operatorname{deg}_{T}$ est fini, c'est-à-dire de redémontrer la proposition 3.17. On notera que si $L$ a le même corps des constantes que $K$, les $d_{\alpha}$ sont tous égaux à un, $\operatorname{deg}_{T_{P_{\Sigma}}}$ est surjectif et donc $\operatorname{deg}_{T}$ également, ce qui redémontre le corollaire 3.21.

\subsection{Préliminaires au calcul des transformées de Fourier}

Soit $v$ une place finie de $K$. Nous choisissons une place $\mathcal{V}$ de $L$ au-dessus de $v$, nous notons $G_{v}$ son groupe de décomposition. On notera encore $\mathcal{V}$ la valuation normalisée de $L$ qui représente $\mathcal{V}$.

Nous notons $\Sigma(1) / G_{v}$ l'ensemble des orbites de $\Sigma(1)$ sous l'action de $G_{v}$, et pour $\alpha \in \Sigma(1) / G$ nous notons $\alpha / G_{v}$ le sous-ensemble de $\Sigma(1) / G_{v}$ des orbites incluses dans $\alpha$. Soit $\Sigma^{G_{v}}$ l'ensemble des cônes de $\Sigma$ globalement invariants sous l'action de $G_{v}$, en d'autres termes les cônes $\sigma$ dont l'ensemble des rayons $\sigma(1)$ est stable sous l'action de $G_{v}$. Pour un cône $\sigma \in \Sigma^{G_{v}}$, nous notons $\sigma(1) / G_{v}$ l'ensemble des orbites de $\sigma(1)$ sous l'action de $G_{v}$, et pour $\alpha \in \Sigma(1) / G$ nous notons $\alpha / G_{v}$ le sous-ensemble de $\sigma(1) / G_{v}$ des orbites incluses dans $\alpha$.

Dans toute la suite de cet texte, nous adoptons pour alléger l'écriture la convention suivante : si $\left(X_{\alpha}\right)_{\alpha \in \Sigma(1) / G}$ est une famille d'objets indexée par $\Sigma(1) / G$, pour tout $\beta \in \Sigma(1) / G_{v}, X_{\beta}$ désigne $X_{\alpha}$ où $\alpha$ est l'unique élément de $\Sigma(1) / G$ contenant $\beta$.

Pour $\beta \in \Sigma(1) / G_{v}$ nous notons $l_{\beta}$ le cardinal de $\beta$ et $\rho_{\beta}^{\prime}$ un générateur d'un élément quelconque de $\beta$. Si $g_{\beta} \in G$ est tel que $\rho_{\beta}^{\prime}=g_{\beta}$. $\rho_{\beta}$, on a donc

$$
l_{\beta}=\frac{\left[G_{v}\right]}{\left[\left(g_{\beta} G_{\beta} g_{\beta}^{-1} \cap G_{v}\right)\right]} .
$$


On pose

$$
\tau_{\beta}=\sum_{\rho \in G_{v} \cdot \rho_{\beta}^{\prime}} \rho,
$$

de sorte que $\tau_{\beta}$ est un élément de $\left(X(T)^{\vee}\right)^{G_{v}}$.

Rappelons que l'on note intrel $(\sigma)$ l'intérieur relatif d'un cône $\sigma$.

\section{Lemme 5.5}

Pour tout $x$ élément de $\left(X(T)^{\vee}\right)^{G_{v}}$, il existe un élément $\sigma \in \Sigma^{G_{v}}$ tel que $x$ s'écrit

$$
x=\sum_{\beta \in \sigma(1) / G_{v}} n_{\beta} \tau_{\beta}
$$

où les $n_{\beta}$ sont des entiers strictement positifs.

En particulier, $\left(X(T)^{\vee}\right)^{G_{v}}$ est recouvert par les ensembles

$$
\operatorname{intrel}(\sigma) \cap\left(X(T)^{\vee}\right)^{G_{v}}
$$

pour $\sigma$ décrivant $\Sigma^{G_{v}}$.

Démonstration : Soit $x \in\left(X(T)^{\vee}\right)^{G_{v}}$. Alors $x$ est dans l'intérieur relatif d'un unique cône $\sigma$ de $\Sigma$. Par ailleurs, pour tout $g \in G_{v}, x=g x$ est dans l'intérieur relatif du cône $g \sigma$ de $\Sigma$, et donc $g \sigma=\sigma$. Ainsi $\sigma \in \Sigma^{G_{v}}$. Le reste du lemme en découle aisément, compte tenu du fait que l'éventail est régulier.

Pour $\alpha \in \Sigma(1) / G$, considérons l'application

$$
\begin{aligned}
G / G_{\alpha} & \longrightarrow\left\{w \in \mathcal{P}_{K_{\alpha}}, v \mid w\right\} \\
g & \left.\longmapsto g^{-1} \cdot \mathcal{V}\right|_{K_{\alpha}}
\end{aligned}
$$

Ce n'est autre que le passage au quotient par l'action de $G_{v}$, d'où une correspondance entre $\alpha / G_{v}$ et les places de $K_{\alpha}$ au-dessus de $v$.

Soit $\beta \in \alpha / G_{v}$ et $w_{\beta}$ la place correspondante. Soit $g_{\beta} \in G$ tel que $\rho_{\beta}^{\prime}=$ $g_{\beta} \cdot \rho_{\alpha}$.

On a

$$
\left[L_{v}: K_{v}\right]=\left[G_{v}\right]
$$

et

$$
\left[L_{\mathcal{V}}:\left(K_{\alpha}\right)_{w_{\beta}}\right]=\left[g_{\beta} G_{\alpha} g_{\beta}^{-1} \cap G_{v}\right]
$$

Ainsi, si $v$ est non ramifiée dans $L$, on a

$$
\left[k_{v}: k_{v}\right]=\left[G_{v}\right]
$$

et

$$
\left[k_{v}: k_{w_{\beta}}\right]=\left[g_{\beta} G_{\alpha} g_{\beta}^{-1} \cap G_{v}\right]
$$


d'où, en combinant les deux égalités précédentes et la formule (5.1),

$$
l_{\beta}=\left[k_{w_{\beta}}: k_{v}\right] .
$$

Ainsi on a

$$
q_{w_{\beta}}=q_{v}^{l_{\beta}} .
$$

Dans le cas fonctionnel (et toujours en supposant $v$ non ramifiée dans $L$ ), on a de plus

$$
q_{w_{\beta}}=q_{K_{\alpha}}^{f_{w_{\beta}}}=q^{d_{\alpha} f_{w_{\beta}}}
$$

soit

$$
f_{v} l_{\beta}=d_{\alpha} f_{w_{\beta}} .
$$

Remarque 5.6 : Bien que nous n'en ayons pas besoin, on peut noter que si $v$ est ramifiée dans $L$, en notant $e_{v}$ l'indice de ramification de $v$ dans $L$ et $e_{\beta}$ l'indice de ramification de $w_{\beta}$ dans $L$, un calcul similaire montre qu'on a

$$
l_{\beta}=\frac{e_{v}}{e_{\beta}}\left[k_{w_{\beta}}: k_{v}\right] \text {. }
$$

soit dans le cas fonctionnel

$$
f_{v} e_{\beta} l_{\beta}=e_{v} d_{\alpha} f_{w_{\beta}}
$$

\subsection{Les transformées de Fourier locales}

Rappelons que $\mathrm{PL}(\Sigma)_{\mathbf{C}}^{G}$ s'identifie canoniquement à $\left(P_{\Sigma}^{G}\right)_{\mathbf{C}}$, et donc à $\mathbf{C}^{\Sigma(1) / G}$ via le choix de la base $\left(D_{\alpha}\right)_{\alpha \in \Sigma(1) / G}$. Nous noterons désormais $\boldsymbol{s}$ ou $\left(s_{\alpha}\right)_{\alpha \in \Sigma(1) / G}$ un élément de $\operatorname{PL}(\Sigma)_{\mathbf{C}}^{G}$.

\subsubsection{Cas d'une place finie quelconque}

\section{Proposition 5.7}

Soit $v$ une place finie de $K$.

1. Pour tout $s \in \mathcal{T}\left(\boldsymbol{R}_{>0}^{\Sigma(1) / G}\right)$ la fonction $H_{v}(-s,$.$) est intégrable sur$ $T\left(K_{v}\right)$.

2. Soit $\chi$ un élément de $\left(T\left(\boldsymbol{A}_{K}\right) / \mathbf{K}(T)\right)^{*}$. La fonction

$$
\boldsymbol{s} \mapsto \int_{T\left(K_{v}\right)} H_{v}(-\boldsymbol{s}, t) \chi_{v}(t) d \mu_{v}(t)
$$

est holomorphe sur $\mathcal{T}\left(\boldsymbol{R}_{>0}^{\Sigma(1) / G}\right)$. On la note $f_{v}(\chi,$.$) .$ 
3. Pour tout compact $\boldsymbol{K}$ de $\boldsymbol{R}_{>0}^{\Sigma(1) / G}$, il existe une constante $C>0$ telle qu'on ait pour tout $\chi \in\left(T\left(\boldsymbol{A}_{K}\right) / \boldsymbol{K}(T)\right)^{*}$ et tout $\boldsymbol{s} \in \mathcal{T}(\boldsymbol{K})$ la majoration

$$
\left|f_{v}(\chi, s)\right| \leqslant C
$$

Démonstration : Pour tout $s \in \mathrm{PL}(\Sigma)_{\mathrm{C}}^{G}$ on a

$$
\left|H_{v}(\boldsymbol{s}, .)\right|=H_{v}(\Re(\boldsymbol{s}), .) .
$$

Comme la fonction $H_{v}(-\boldsymbol{s},$.$) est T\left(\mathcal{O}_{v}\right)$-invariante, elle induit une fonction sur $T\left(K_{v}\right) / T\left(\mathcal{O}_{v}\right)$, qui sera notée de façon identique. Pour $s \in \operatorname{PL}(\Sigma)_{\mathbf{C}}^{G}$ on a

$$
\begin{aligned}
& \int_{T\left(K_{v}\right)}\left|H_{v}(-\boldsymbol{s}, t)\right| d \mu_{v}(t) \\
= & \int_{T\left(K_{v}\right)} H_{v}(-\Re(\boldsymbol{s}), t) d \mu_{v}(t) \\
= & \left(\int_{T\left(\mathcal{O}_{v}\right)} d \mu_{v}\right) \sum_{t \in T\left(K_{v}\right) / T\left(\mathcal{O}_{v}\right)} H_{v}(-\Re(\boldsymbol{s}), t) .
\end{aligned}
$$

Soit $\mathcal{V}$ une place de $L$ divisant $v$ et $G_{v}$ son groupe de décomposition. Soit $t \in T\left(K_{v}\right) / T\left(\mathcal{O}_{v}\right)$. Alors $\operatorname{deg}_{T, L, \mathcal{V}}(t)$ est un élément de $\left(X(T)^{\vee}\right)^{G_{v}}$. D'après le lemme 5.5 il existe un unique élément $\sigma \in \Sigma^{G_{v}}$ tel que $\operatorname{deg}_{T, L, \mathcal{V}}(t)$ s'écrit

$$
\operatorname{deg}_{T, L, \mathcal{V}}(t)=\sum_{\beta \in \sigma(1) / G_{v}} n_{\beta} \tau_{\beta}
$$

où les $n_{\beta}$ sont des entiers strictement positifs. On a alors

$$
H_{v}(-s, t)=q_{v}^{-\frac{1}{e_{v}}} \sum_{\beta \in \sigma(1) / G_{v}} n_{\beta} l_{\beta} s_{\beta}
$$

Ainsi, compte tenu du fait que $\operatorname{deg}_{T, L, \mathcal{V}}: T\left(K_{v}\right) / T\left(\mathcal{O}_{v}\right) \rightarrow\left(X(T)^{\vee}\right)^{G_{v}}$ est injectif et du lemme 5.5, on a

$$
\sum_{t \in T\left(K_{v}\right) / T\left(\mathcal{O}_{v}\right)} H_{v}(-\Re(\boldsymbol{s}), t) \leqslant \sum_{\sigma \in \Sigma^{G} v} \prod_{\beta \in \sigma(1) / G_{v}} \sum_{\left(n_{\beta}\right) \in \mathbf{Z}_{>0}^{\sigma(1) / G_{v}}} q_{v}^{-\frac{1}{e_{v}}}{ }_{\beta \in \sigma(1) / G_{v}}^{\sum_{\beta} n_{\beta} l_{\beta} s_{\beta}}
$$

Le membre de gauche est visiblement fini dès qu'on a $\Re\left(s_{\alpha}\right)>0$ pour tout $\alpha$. La proposition en découle facilement. 
Remarque 5.8: Pour $\chi \in\left(T\left(\mathbf{A}_{K}\right) / \mathbf{K}(T)\right)^{*}$, la démonstration montre que pour tout $\boldsymbol{s} \in \mathcal{T}\left(\mathbf{R}_{>0}^{\Sigma(1) / G}\right)$, la série

$$
\sum_{t \in T\left(K_{v}\right) / T\left(\mathcal{O}_{v}\right)} \chi(t) H_{v}(-s, t)
$$

est absolument convergente et qu'on a

$$
f_{v}(\chi, s)=\left(\int_{T\left(\mathcal{O}_{v}\right)} d \mu_{v}\right) \sum_{t \in T\left(K_{v}\right) / T\left(\mathcal{O}_{v}\right)} \chi(t) H_{v}(-s, t) .
$$

\subsubsection{Cas arithmétique}

\section{Lemme 5.9}

On se place dans le cas arithmétique. Soit $v$ une place de K. On a

$$
\forall y \in X(T)_{\boldsymbol{R}}^{G}, \quad \forall t \in T\left(K_{v}\right), \quad\left(\chi_{y}\right)_{v}(t)=H_{v}\left(i \gamma_{\boldsymbol{R}}(y), t\right) .
$$

Démonstration: Soit $\mathcal{V}$ une place de $L$ divisant $v$. Pour $y \in X(T)_{\mathbf{R}}^{G}$ et $t \in T\left(K_{v}\right)$ on a, compte tenu de (5.7), de la définition (4.20) de $H_{v}$ et du lemme 4.3 (respectivement 4.4) si $v$ est finie (respectivement archimédienne) :

$$
\begin{aligned}
\left(\chi_{y}\right)_{v}(t) & =\exp \left(i \log \left(q_{v}\right)\left\langle y, \operatorname{deg}_{T, v}(t)\right\rangle\right) \\
& =\exp \left(i \log \left(q_{v}\right)\left\langle\gamma_{\mathbf{R}}(y), t\right\rangle_{\Sigma, v}\right) \\
& =\exp \left(\log \left(q_{v}\right)\left\langle i \gamma_{\mathbf{R}}(y), t\right\rangle_{\Sigma, v}\right) \\
& =H_{v}\left(i \gamma_{\mathbf{R}}(y), t\right)
\end{aligned}
$$

d'où le résultat.

\section{Corollaire 5.10}

On a

$$
\begin{aligned}
\forall y \in X(T)_{\boldsymbol{R}}^{G}, \quad \forall \boldsymbol{s} \in \operatorname{PL}(\Sigma)_{\boldsymbol{C}}^{G}, \quad \forall t \in T\left(K_{v}\right), \\
H_{v}(\boldsymbol{s}, t)\left(\chi_{y}\right)_{v}(t)=H_{v}\left(\boldsymbol{s}+i \gamma_{\boldsymbol{R}}(y), t\right) .
\end{aligned}
$$

Corollaire 5.11

Soit $v$ une place de $K$ et $\chi$ un élément de $\left(T\left(\boldsymbol{A}_{K}\right) / \mathbf{K}(T)\right)^{*}$. Alors on a

$$
\begin{aligned}
\forall y \in X(T)_{\boldsymbol{R}}^{G}, \quad \forall \boldsymbol{s} \in \mathcal{T}\left(\boldsymbol{R}_{>0}^{\Sigma(1) / G}\right), & \\
& f_{v}\left(\chi \cdot \chi_{y}, \boldsymbol{s}\right)=f_{v}\left(\chi, \boldsymbol{s}-i \gamma_{\boldsymbol{R}}(y)\right) .
\end{aligned}
$$


Dans le reste de cette section, on s'intéresse aux propriétés des transformées de Fourier locales aux places archimédiennes. Ces propriétés seront cruciales pour appliquer la formule de Poisson dans le cas arithmétique.

Soit $v \in \mathcal{P}_{K, \infty}$ et $\mathcal{V}$ une place de $L$ divisant $v$, de groupe de décomposition $G_{v}$. D'après le lemme $3.13, T\left(K_{v}\right) / T\left(\mathcal{O}_{v}\right)$ s'identifie naturellement à $\left(X(T)_{\mathbf{R}}^{\vee}\right)^{G_{v}}$.

On note $\Sigma_{\max }^{G_{v}}$ l'ensemble des cônes de $\Sigma^{G_{v}}$ de dimension maximale. Soit $\sigma \in \Sigma_{\max }^{G_{v}}$. Pour tout $j \in \sigma(1) / G_{v}$, soit $l_{j}$ le cardinal de $j$ (ainsi $l_{j}=1$ ou 2), $\rho_{j}$ un générateur d'un élément de $j$, et

$$
\tau_{j}=\sum_{\rho \in G_{v}} \rho .
$$

Si $\alpha$ est l'élément de $\Sigma(1) / G$ tel que $j \subset \alpha$, la notation $s_{j}$ désigne $s_{\alpha}$.

La proposition suivante donne les propriétés et estimations nécessaires pour les transformées de Fourier locales aux places archimédiennes.

\section{Proposition 5.12}

Soit $v$ un élément de $\mathcal{P}_{K, \infty}$.

1. Pour tout $\boldsymbol{s} \in \mathcal{T}\left(\boldsymbol{R}_{>0}^{\Sigma(1) / G}\right)$ la fonction $H_{v}(-\boldsymbol{s},$.$) est intégrable sur$ $T\left(K_{v}\right)$.

2. Soit $\chi$ un élément de $\left(T\left(\boldsymbol{A}_{K}\right) / \boldsymbol{K}(T)\right)^{*}$. La fonction

$$
s \mapsto \int_{T\left(K_{v}\right)} H_{v}(-s, t) \chi_{v}(x) d \mu_{v}(x)
$$

est holomorphe sur $\mathcal{T}\left(\boldsymbol{R}_{>0}^{\Sigma(1) / G}\right)$. On la note $f_{v}(\chi,$.$) .$

3. On a, pour tout élément $\chi$ de $\left(T\left(\boldsymbol{A}_{K}\right) / \boldsymbol{K}(T)\right)^{*}$,

$$
\forall \boldsymbol{s} \in \mathcal{T}\left(\boldsymbol{R}_{>0}^{\Sigma(1) / G}\right), \quad f_{v}(\chi, s)=\sum_{\sigma \in \Sigma_{\max }^{G v}} \frac{1}{\prod_{j \in \sigma(1) / G_{v}} \frac{l_{j}}{\left[L_{\mathcal{V}}: K_{v}\right]}\left(s_{j}+i\left\langle\rho_{j}, \chi_{v}\right\rangle\right)} .
$$

4. Soit $\varepsilon$ vérifiant $0<\varepsilon<1, \boldsymbol{K}$ un compact de $\boldsymbol{R}_{>1-\varepsilon}^{\Sigma(1) / G}$ et $\|$.$\| une norme$ sur $\left(T\left(K_{v}\right) / T\left(\mathcal{O}_{v}\right)\right)^{*}$. Il existe alors une constante $C>0$ telle qu'on ait, pour tout $v$ de $\mathcal{P}_{K, \infty}$, et tout $\chi \in\left(T\left(\boldsymbol{A}_{K}\right) / \mathbf{K}(T)\right)^{*}$,

$$
\forall \boldsymbol{s} \in \mathcal{T}(\boldsymbol{K}), \quad\left|f_{v}(\chi, \boldsymbol{s})\right| \leqslant \frac{C}{1+\left\|\chi_{v}\right\|} \sum_{\sigma \in \Sigma_{\max }^{G v}} \frac{1+\sum_{j \in \sigma(1) / G_{v}}\left|\Im\left(s_{j}\right)\right|}{\prod_{j \in \sigma(1) / G_{v}}\left(1+\left|\left\langle\rho_{j}, \chi_{v}\right\rangle+\Im\left(s_{j}\right)\right|\right)}
$$


Démonstration : Dans tout ce qui suit, on identifie $T\left(K_{v}\right) / T\left(\mathcal{O}_{v}\right)$ à $\left(X(T)^{\vee}\right)_{\mathbf{R}}^{G_{v}}$ et donc $T\left(K_{v}\right) / T\left(\mathcal{O}_{v}\right)^{*}$ à $X(T)_{\mathbf{R}}^{G_{v}}$.

On commence par remarquer la chose suivante. Soit $t \in T\left(K_{v}\right) / T\left(\mathcal{O}_{v}\right)$. Alors il existe un élément $\sigma$ de $\Sigma_{\max }^{G_{v}}$ et des réels positifs $\left(t_{j}\right)_{j \in \sigma(1) / G_{v}}$ tels qu'on ait

$$
t=\sum_{j \in \sigma(1) / G_{v}} t_{j} \tau_{j}
$$

D'après (4.13) et (4.20), on a alors, pour tout $\left(s_{\alpha}\right) \in \mathbf{C}^{\Sigma(1)}$,

$$
H_{v}(s, t)=\exp \left(\frac{1}{\left[L_{\mathcal{V}}: K_{v}\right]} \sum_{j \in \sigma(1) / G_{v}} t_{j} l_{j} s_{j}\right)
$$

On a également, pour tout $\chi_{v} \in T\left(K_{v}\right) / T\left(\mathcal{O}_{v}\right)^{*}$,

$$
\chi_{v}(t)=\exp \left(i\left\langle t, \chi_{v}\right\rangle\right)=\exp \left(i \sum_{j \in \sigma(1) / G_{v}} t_{j} l_{j}\left\langle\rho_{j}, \chi_{v}\right\rangle\right) .
$$

Pour tout $\boldsymbol{s} \in \mathbf{C}^{\Sigma(1) / G}$ on a $\left|H_{v}(\boldsymbol{s},).\right|=H_{v}(\Re(\boldsymbol{s}),$.$) . Comme la fonction$ $H_{v}(-\boldsymbol{s},$.$) est T\left(\mathcal{O}_{v}\right)$-invariante, elle induit une fonction sur $T\left(K_{v}\right) / T\left(\mathcal{O}_{v}\right)$, qui sera notée de façon identique.

Pour $\boldsymbol{s} \in \mathbf{C}^{\Sigma(1) / G}$, on a alors

$$
\begin{aligned}
\int_{T\left(K_{v}\right)}\left|H_{v}(-\boldsymbol{s}, t)\right| d \mu_{v}(t) & =\int_{T\left(K_{v}\right)} H_{v}(-\Re(\boldsymbol{s}), t) d \mu_{v}(t) \\
& =\int_{T\left(K_{v}\right) / T\left(\mathcal{O}_{v}\right)} H_{v}(-\Re(\boldsymbol{s}), t) d t
\end{aligned}
$$

où $d t$ est la mesure de Lebesgue sur $T\left(K_{v}\right) / T\left(\mathcal{O}_{v}\right) \stackrel{\sim}{\rightarrow}\left(X(T)_{\mathbf{R}}^{\vee}\right)^{G_{v}}$, normalisée par le réseau $\left(X(T)^{\vee}\right)^{G_{v}}$

Comme $\left(X(T)_{\mathbf{R}}^{\vee}\right)^{G_{v}}$ est recouvert par les éléments de $\Sigma_{\max }^{G_{v}}$ et que l'intersection de deux éléments distincts de $\Sigma_{\max }^{G_{v}}$ est de mesure de Lebesgue nulle, on a

$$
\int_{T\left(K_{v}\right) / T\left(\mathcal{O}_{v}\right)} H_{v}(-\Re(\boldsymbol{s}), t) d t=\sum_{\sigma \in \Sigma_{\max }^{G_{v}}} \int_{\sigma} H_{v}(-\Re(\boldsymbol{s}), t) d t
$$

Pour $\sigma \in \Sigma_{\max }^{G_{v}}$, les $\left(\tau_{j}\right)_{j \in \sigma(1) / G_{v}}$ engendrent $\sigma$ et forment une base de $\left(X(T)^{\vee}\right)^{G_{v}}$. Ainsi, si on identifie $\sigma$ à $\mathbf{R}_{\geqslant 0}^{\sigma(1) / G_{v}}$ au moyen de la base $\left(\tau_{j}\right)$, la restriction de 
la mesure $d x$ à $\sigma$ s'identifie à la mesure produit $\underset{j \in \sigma(1) / G_{v}}{\otimes} d x_{j}$ sur $\mathbf{R}_{\geqslant 0}^{\sigma(1) / G_{v}}$. On a donc

$$
\int_{\sigma} H_{v}(-\Re(\boldsymbol{s}), t) d t=\int_{\mathbf{R}_{\geqslant 0}^{\sigma(1) / G_{v}}} H_{v}\left(-\Re(\boldsymbol{s}), \sum t_{j} \tau_{j}\right) \underset{j \in \sigma(1) / G_{v}}{\otimes} d t_{j}
$$

soit

$$
\int_{T\left(K_{v}\right) / T\left(\mathcal{O}_{v}\right)} H_{v}(-\Re(\boldsymbol{s}), t) d t=\sum_{\sigma \in \Sigma_{\max }^{G_{v}}} \int_{\mathbf{R}_{\geqslant 0}^{\sigma(1) / G_{v}}} \exp \left(\frac{\sum-t_{j} l_{j} \Re\left(s_{j}\right)}{\left[L_{\mathcal{V}}: K_{v}\right]}\right) \underset{j \in \sigma(1) / G_{v}}{\otimes} d t_{j}
$$

Cette dernière expression est finie dès que $\Re\left(s_{j}\right)>0$ pour tout $j \in \sigma(1) / G_{v}$ et tout $\sigma \in \Sigma_{\text {max }}^{G_{v}}$, c'est-à-dire dès que $\Re\left(s_{\alpha}\right)>0$ pour tout $\alpha \in \Sigma(1) / G$. Elle vaut alors

$$
\sum_{\sigma \in \Sigma_{\max }^{G v}} \prod_{j \in \sigma(1) / G_{v}} \frac{1}{\frac{l_{j}}{\left[L_{\mathcal{v}}: K_{v}\right]} \Re\left(s_{j}\right)} .
$$

On en déduit les points 1 et 2 .

Montrons le point 3. Soit $\boldsymbol{s} \in \mathcal{T}\left(\mathbf{R}_{>0}^{\Sigma(1) / G}\right)$. On a

$$
\begin{aligned}
& \int_{T\left(K_{v}\right)} \chi_{v}(x) H_{v}(-s, t) d \mu_{v}(t) \\
& =\int_{T\left(K_{v}\right) / T\left(\mathcal{O}_{v}\right)} \chi_{v}(t) H_{v}(-s, t) d t \\
& =\sum_{\sigma \in \Sigma_{\max }^{G v}} \int_{\sigma} \chi_{v}(t) H_{v}(-s, t) d t \\
& =\sum_{\sigma \in \Sigma_{\max }^{G v}} \int_{\mathbf{R}_{\geqslant 0}^{\sigma(1) / G_{v}}} \chi_{v}\left(\sum t_{j} \tau_{j}\right) H_{v}\left(-s, \sum t_{j} \tau_{j}\right) \underset{j \in \sigma(1) / G_{v}}{\otimes} d t_{j} \\
& =\sum_{\sigma \in \Sigma_{\max }^{G_{v}}} \int_{\mathbf{R}_{\geqslant 0}^{\sigma(1) / G_{v}}} \exp \left(\frac{\sum t_{j} l_{j}\left(s_{j}+i\left\langle\rho_{j}, \chi_{v}\right\rangle\right)}{\left[L_{\mathcal{V}}: K_{v}\right]}\right) \underset{j \in \sigma(1) / G_{v}}{\otimes} d t_{j} \\
& =\sum_{\sigma \in \Sigma_{\max }^{G_{v}}} \prod_{j \in \sigma(1) / G_{v}} \frac{1}{\frac{l_{j}}{\left[L_{v}: K_{v}\right]}\left(s_{j}+i\left\langle\rho_{j}, \chi_{v}\right\rangle\right)} .
\end{aligned}
$$

Montrons le point 4 . Soit $\left(e_{l}\right)_{l \in L}$ une base de $\left(X(T)^{\vee}\right)^{G_{v}}$ et $\left(e_{l}^{\vee}\right)$ sa base duale. Soit $\boldsymbol{s} \in \mathcal{T}\left(\mathbf{R}_{>0}^{\Sigma(1) / G}\right)$ et $\chi \in\left(T\left(\mathbf{A}_{K}\right) / \mathbf{K}(T)\right)^{*}$. Soit $g$ (respectivement 
h) la fonction de $\mathbf{R}^{L}$ dans $\mathbf{C}$ donnée par

$$
\forall\left(t_{l}\right) \in \mathbf{R}^{L}, \quad g\left(t_{l}\right)=H_{v}\left(-s, \sum_{l \in L} t_{l} e_{l}\right),
$$

respectivement

$$
\forall\left(t_{l}\right) \in \mathbf{R}^{L}, \quad h\left(t_{l}\right)=\chi_{v}\left(\sum_{l \in L} t_{l} e_{l}\right)=\exp \left(i \sum_{l \in L} t_{l}\left\langle e_{l}, \chi_{v}\right\rangle\right) .
$$

On a ainsi , pour $l \in L$,

$$
\frac{\partial}{\partial t_{l}} h(t)=i\left\langle e_{l}, \chi_{v}\right\rangle h(t)
$$

Pour tout $l$ vérifiant $\left\langle e_{l}, \chi_{v}\right\rangle \neq 0$ on obtient en intégrant par parties

$$
\begin{aligned}
f_{v}(\chi, s) & =\int_{\mathbf{R}^{L}} g(t) h(t) d t \\
& =-\frac{1}{i\left\langle e_{l}, \chi_{v}\right\rangle} \int_{\mathbf{R}^{L}} h(t) \frac{\partial}{\partial t_{l}} g(t) d t \\
& =-\sum_{\sigma \in \Sigma_{\max }^{G}} \frac{1}{i\left\langle e_{l}, \chi_{v}\right\rangle} \int_{\sigma} h(t) \frac{\partial}{\partial t_{l}} g(t) d t .
\end{aligned}
$$

La restriction de $g$ à $\sigma \in \Sigma_{\max }^{G_{v}}$ s'écrit (en notant $\left(\tau_{j}^{\vee}\right)_{j \in \sigma(1) / G_{v}}$ la base duale $\left.\operatorname{de}\left(\tau_{j}\right)_{j \in \sigma(1) / G_{v}}\right)$

$$
\begin{aligned}
g(t) & =\exp \left(-\frac{1}{\left[L_{\mathcal{V}}: K_{v}\right]} \sum_{j \in \sigma(1) / G_{v}}\left\langle\sum_{l \in L} t_{l} e_{l}, \tau_{j}^{\vee}\right\rangle l_{j} s_{j}\right) \\
& =\exp \left(-\frac{1}{\left[L_{\mathcal{V}}: K_{v}\right]} \sum_{l \in L} t_{l} \sum_{j \in \sigma(1) / G_{v}}\left\langle e_{l}, \tau_{j}^{\vee}\right\rangle l_{j} s_{j}\right)
\end{aligned}
$$

et finalement sur $\sigma$ on a

$$
\frac{\partial}{\partial t_{l}} g(t)=-\left(\frac{1}{\left[L_{\mathcal{V}}: K_{v}\right]} \sum_{j \in \sigma(1) / G_{v}}\left\langle e_{l}, \tau_{j}^{\vee}\right\rangle l_{j} s_{j}\right) g(t)
$$


On en déduit

$$
i\left\langle e_{l}, \chi_{v}\right\rangle f_{v}(\chi, s)=\sum_{\sigma \in \Sigma_{\max }^{G v}}\left(\sum_{j \in \sigma(1) / G_{v}}\left\langle e_{l}, \tau_{j}^{\vee}\right\rangle \frac{l_{j}}{\left[L_{\mathcal{V}}: K_{v}\right]} s_{j}\right) \int_{\sigma} h(t) g(t) d t
$$

soit

$$
\begin{aligned}
& i\left\langle e_{l}, \chi_{v}\right\rangle f_{v}(\chi, s) \\
= & \sum_{\sigma \in \Sigma_{\max }^{G}}\left(\sum_{j \in \sigma(1) / G_{v}}\left\langle e_{l}, \tau_{j}^{\vee}\right\rangle \frac{l_{j}}{\left[L_{\mathcal{V}}: K_{v}\right]} s_{j}\right) \prod_{j \in \sigma(1) / G_{v}} \frac{1}{\frac{l_{j}}{\left[L_{v}: K_{v}\right]}\left(s_{j}+i\left\langle\rho_{j}, \chi_{v}\right\rangle\right)} .
\end{aligned}
$$

En prenant les modules dans (5.37) et (5.48) pour tous les $l$ tels que $\left\langle e_{l}, \chi_{v}\right\rangle \neq$ 0 et en ajoutant le tout, on obtient après une majoration évidente

$$
\begin{aligned}
& \left(1+\sum_{l \in L}\left|\left\langle e_{l}, \chi_{v}\right\rangle\right|\right)\left|f_{v}(\chi, s)\right| \\
\leqslant & \sum_{\sigma \in \Sigma_{\max }^{G v}}\left(1+\sum_{l \in L}\left|\sum_{j \in \sigma(1) / G_{v}}\left\langle e_{l}, \tau_{j}^{\vee}\right\rangle \frac{l_{j}}{\left[L_{\mathcal{V}}: K_{v}\right]} s_{j}\right|\right) \prod_{j \in \sigma(1) / G_{v}} \frac{1}{\left|\frac{l_{j}}{\left[L_{\mathcal{v}}: K_{v}\right]}\left(s_{j}+i\left\langle\rho_{j}, \chi_{v}\right\rangle\right)\right|} .
\end{aligned}
$$

Soit $\|.\|_{e}$ la norme sur $X(T)_{\mathbf{C}}^{G_{v}}$ définie par

$$
\forall x \in X(T)_{\mathbf{C}}^{G_{v}}, \quad\|x\|_{e}=\sum_{l \in L}\left|\left\langle e_{l}, x\right\rangle\right|
$$

De (5.49), on tire pour tout $s \in \mathcal{T}\left(\mathbf{R}_{>0}^{\Sigma(1) / G}\right)$ et tout $\chi_{v} \in T\left(K_{v}\right) / T\left(\mathcal{O}_{v}\right)^{*}$ la majoration

$$
\left|f_{v}\left(\chi_{v}, \boldsymbol{s}\right)\right| \leqslant \frac{2^{\operatorname{rg}(X(T))}}{1+\left\|\chi_{v}\right\|_{e}} \sum_{\sigma \in \Sigma_{\max }^{G v}} \prod_{j \in \sigma(1) / G_{v}} \frac{1+\left\|\sum_{j \in \sigma(1) / G_{v}} \frac{l_{j}}{\left[L_{v}: K_{v}\right]} s_{j} \tau_{j}^{\vee}\right\| \|_{e}}{\left.\mid s_{j}+i\left\langle\rho_{j}, \chi_{v}\right\rangle\right) \mid} .
$$

L'équivalence des normes sur $X(T)_{\mathrm{C}}^{G_{v}}$ montre l'existence d'une constante $C_{1}$ vérifiant, pour tout $\boldsymbol{s} \in \mathbf{C}^{\Sigma(1) / G}$,

$$
\left\|\sum_{j \in \sigma(1) / G_{v}} \frac{l_{j}}{\left[L_{\mathcal{V}}: K_{v}\right]} s_{j} \tau_{j}^{\vee}\right\|_{e} \leqslant C_{1} \sum_{j \in \sigma(1) / G_{v}}\left|\frac{l_{j}}{\left[L_{\mathcal{V}}: K_{v}\right]} s_{j}\right| \leqslant C_{1} \sum_{j \in \sigma(1) / G_{v}}\left|s_{j}\right| .
$$


Il existe par ailleurs une constante $C_{2}>0$ telle qu'on ait pour tout $\boldsymbol{s} \in \mathcal{T}(\mathbf{K})$, pour tout $\sigma \in \Sigma_{\max }^{G_{v}}$ et tout $j \in \sigma(1) / G_{v}$

$$
\left.\mid s_{j}+i\left\langle\rho_{j}, \chi_{v}\right\rangle\right) \mid \geqslant C_{2}\left(1+\left|\left\langle\rho_{j}, \chi_{v}\right\rangle+\Im\left(s_{j}\right)\right|\right) .
$$

Le point 4 s'en déduit aisément.

On notera

$$
f_{\infty}(\chi, .)=\prod_{v \in \mathcal{P}_{K, \infty}} f_{v}(\chi, .)
$$

On pose

$$
X(T)_{\mathbf{R}, \infty}=\bigoplus_{v \in \mathcal{P}_{K, \infty}} X(T)_{\mathbf{R}}^{G_{v}} .
$$

Soit $\chi$ un élément de $\left(T\left(\mathbf{A}_{K}\right) / \mathbf{K}(T)\right)^{*}$. Pour toute place $v$ de $K$, $\chi_{v}$ est alors un élément de $\left(T\left(K_{v}\right) / T\left(\mathcal{O}_{v}\right)\right)^{*}$. Si $v$ est archimédienne, on a un isomorphisme

$$
\operatorname{deg}_{T, v}^{*}: \mathbf{X}(T)_{\mathbf{R}}^{G_{v}} \stackrel{\sim}{\longrightarrow}\left(T\left(K_{v}\right) / T\left(\mathcal{O}_{v}\right)\right)^{*}
$$

\section{Définition 5.13}

Le morphisme «type à l'infini» est l'application qui à $\chi \in\left(T\left(\boldsymbol{A}_{K}\right) / \boldsymbol{K}(T)\right)^{*}$ associe

$$
\chi_{\infty} \stackrel{\text { déf }}{=}\left(\left(\operatorname{deg}_{T, v}^{*}\right)^{-1} \chi_{v}\right) \in X(T)_{\mathbf{R}, \infty} .
$$

Soit $\Sigma^{\infty}$ l'éventail produit des $\Sigma^{G_{v}}$ pour $v \in \mathcal{P}_{K, \infty}$ : c'est l'éventail de $X(T)_{\mathbf{R}, \infty}$ dont les cônes sont des produits des cônes des éventails $\Sigma^{G_{v}}$ pour $v \in \mathcal{P}_{K, \infty}$. L'ensemble $\Sigma_{\max }^{\infty}$ des cônes de $\Sigma^{\infty}$ de dimension maximale est donc l'ensemble des produits d'éléments de $\Sigma_{\max }^{G_{v}}$ pour $v \in \mathcal{P}_{K, \infty}$.

\section{Corollaire 5.14}

Soit $\varepsilon$ vérifiant $0<\varepsilon<1$ et $\boldsymbol{K}$ un compact de $\boldsymbol{R}_{>1-\varepsilon}^{\Sigma(1) / G}$. Il existe une constante $C>0$ telle qu'on ait

$$
\begin{aligned}
& \forall \boldsymbol{s} \in \mathcal{T}(\boldsymbol{K}), \quad \forall \chi \in\left(T\left(\boldsymbol{A}_{K}\right) / \mathbf{K}(T)\right)^{*}, \\
&\left|f_{\infty}(\chi, s)\right| \leqslant \frac{C}{1+|| \chi_{\infty} \|} \sum_{\widetilde{\sigma} \in \Sigma_{\max }^{\infty}} \frac{1+\sum_{i \in \widetilde{\sigma}(1)}\left|\Im\left(s_{i}\right)\right|}{\prod_{i \in \widetilde{\sigma}(1)}\left(1+\left|\left\langle\rho_{i}, \chi_{\infty}\right\rangle+\Im\left(s_{i}\right)\right|\right)}
\end{aligned}
$$

Démonstration : On applique le point 4 de la proposition 5.12 en prenant pour éventail $\Sigma^{\infty}$. 


\subsubsection{Cas fonctionnel}

On introduit d'abord quelques définitions.

On rappelle qu'au moyen de la base $\left(D_{\alpha}\right)_{\alpha \in \Sigma(1) / G}$ on identifie $P_{\Sigma}^{G}$ à $\mathbf{Z}^{\Sigma(1) / G}$. Ceci permet d'identifier $\left(P_{\Sigma}^{G}\right)_{\mathbf{C}}$ à $\mathbf{C}^{\Sigma(1) / G}$ et $\left(P_{\Sigma}^{G}\right)_{\mathbf{C}^{\times}}$à $\left(\mathbf{C}^{\times}\right)^{\Sigma(1) / G}$ (donc à un sous-ensemble de $\left.\mathbf{C}^{\Sigma(1) / G}\right)$.

Dans toute la suite de ce texte, le terme série formelle désignera un élément de $\mathbf{C}\left[\left[z_{\alpha}\right]\right]_{\alpha \in \Sigma(1) / G}$ et le terme monôme un monôme de $\mathbf{C}\left[z_{\alpha}\right]_{\alpha \in \Sigma(1) / G}$. On identifiera un monôme à la fonction $\mathbf{C}^{\Sigma(1) / G} \rightarrow \mathbf{C}$ qu'il induit.

Soit

$$
P=\sum_{\left(n_{\alpha}\right) \in \mathbf{N}^{\Sigma(1) / G}} a_{\left(n_{\alpha}\right)} \prod z_{\alpha}^{n_{\alpha}}
$$

une série formelle. Soit $\boldsymbol{z}$ un élément de $\left(\mathbf{C}^{\times}\right)^{\Sigma(1) / G}$ tel que la série définissant $P(\boldsymbol{z})$ converge absolument. Si on voit $\boldsymbol{z}$ comme un élément de $\left(P_{\Sigma}^{G}\right)_{\mathbf{C}^{\times}}$, on a donc

$$
P(\boldsymbol{z})=\sum_{\left(n_{\alpha}\right) \in \mathbf{N}^{\Sigma(1) / G}} a_{\left(n_{\alpha}\right)} \prod\left\langle\boldsymbol{z}, D_{\alpha}^{\vee}\right\rangle^{n_{\alpha}}
$$

\section{Lemme 5.15}

Soit $\left(n_{\alpha}\right)$ un élément de $\boldsymbol{N}^{\Sigma(1) / G}$. Alors l'application qui à $\boldsymbol{z} \in\left(P_{\Sigma}^{G}\right)_{\boldsymbol{C}^{\times}}=$ $\left(\boldsymbol{C}^{\times}\right)^{\Sigma(1) / G}$ associe $\left\langle\boldsymbol{z}, \sum n_{\alpha} D_{\alpha}^{\vee}\right\rangle$ s'étend en un unique monôme sur $\boldsymbol{C}^{\Sigma(1) / G}$.

\section{Lemme 5.16}

Soit

$$
P=\sum_{\left(n_{\alpha}\right) \in \mathbf{N}^{\Sigma(1) / G}} a_{\left(n_{\alpha}\right)} \prod z_{\alpha}^{n_{\alpha}}
$$

une série formelle. Alors pour tout $\boldsymbol{z} \in X(T)_{C^{\times}}^{G}$ tel que la série définissant $P\left(\gamma_{\boldsymbol{C}^{\times}}(\boldsymbol{z})\right)$ converge absolument, on a

$$
P\left(\gamma_{\boldsymbol{C}^{\times}}(\boldsymbol{z})\right)=\sum_{\left(n_{\alpha}\right) \in \boldsymbol{N}^{\Sigma(1) / G}} a_{\left(n_{\alpha}\right)} \prod\left\langle\boldsymbol{z}, \rho_{\alpha}\right\rangle^{n_{\alpha}} .
$$

Démonstration: Compte tenu de (5.60), il suffit de remarquer que pour $\alpha \in \Sigma(1) / G$ on a

$$
\left\langle\gamma_{\mathbf{C}^{\times}}(\boldsymbol{z}), D_{\alpha}^{\vee}\right\rangle=\left\langle\boldsymbol{z}, \gamma^{\vee}\left(D_{\alpha}^{\vee}\right)\right\rangle
$$

et que, d'après (4.15), on a $\gamma^{\vee}\left(D_{\alpha}^{\vee}\right)=\rho_{\alpha}$.

\section{Définition 5.17}

Soit $M$ un sous-groupe de $\left(X(T)^{G}\right)^{\vee}$. 
1. Soit $\left(n_{\alpha}\right) \in \mathbf{N}^{\Sigma(1) / G}$. Le monôme $\prod_{\alpha \in \Sigma(1) / G} z_{\alpha}^{n_{\alpha}}$ est dit $M$-compatible si on a

$$
\gamma^{\vee}\left(\sum n_{\alpha} D_{\alpha}^{\vee}\right) \in M
$$

ce qui équivaut d'après (4.15) à la condition

$$
\sum n_{\alpha} \rho_{\alpha} \in M
$$

2. Une série formelle est dite $M$-compatible si les monômes qui apparaissent dans son écriture sont $M$-compatibles.

On a le lemme élémentaire suivant.

\section{Lemme 5.18}

Soit $M$ un sous-groupe de $\left(X(T)^{G}\right)^{\vee}$.

1. Un monôme $f$ est $M$-compatible si et seulement s'il existe un élément $m$ de $M$ vérifiant

$$
\forall \boldsymbol{z} \in X(T)_{\boldsymbol{C}^{\times}}^{G}, \quad f\left(\gamma_{\boldsymbol{C}^{\times}}(\boldsymbol{z})\right)=\langle\boldsymbol{z}, m\rangle
$$

2. Tout produit ou somme de série formelles $M$-compatibles est $M$-compatible.

\section{Lemme 5.19}

Pour tout $\alpha \in \Sigma(1) / G$, le monôme $z_{\alpha}^{d_{\alpha}}$ est $\mathcal{D}_{T}^{0}$-compatible.

Démonstration : C'est immédiat d'après la définition et le fait, donné par le lemme 5.3, que $d_{\alpha} \rho_{\alpha}$ est dans $\mathcal{D}_{T}^{0}$.

\section{Définition 5.20}

Le rayon de convergence d'une série formelle $P$ est le plus grand réel positif $R$ tel que pout tout $\left(z_{\alpha}\right) \in C^{\Sigma(1) / G}$ vérifiant $\operatorname{Max}_{\alpha}\left|z_{\alpha}\right|<R$, la série définissant $P\left(z_{\alpha}\right)$ est absolument convergente.

\section{Lemme 5.21}

Soit $v$ une place de $K$ vérifiant l'hypothèse 4.8 .

1. On a

$$
\forall \boldsymbol{z} \in X(T)_{\boldsymbol{U}}^{G}, \quad \forall t \in T\left(K_{v}\right), \quad\left(\chi_{\boldsymbol{z}}\right)_{v}(t)=\mathfrak{H}_{v}\left(\gamma_{\boldsymbol{C}^{\times}}(\boldsymbol{z}), t\right) .
$$

2. Pour tout $t \in T\left(K_{v}\right)$, la fonction $\mathfrak{H}_{v}(., t)$ s'étend en un monôme qui est $\mathcal{D}_{T}$-compatible. Ce monôme est une constante si et seulement si $t$ est dans $T\left(\mathcal{O}_{v}\right)$. 
Démonstration: Pour $\boldsymbol{z} \in X(T)_{\mathbf{C}^{\times}}^{G}$, et $t \in T\left(K_{v}\right)$, on a, compte tenu du lemme 3.11 et de la définition (4.41) de $\mathfrak{H}_{v}$

$$
\begin{aligned}
\left\langle\boldsymbol{z}, \operatorname{deg}_{T, v}(t)\right\rangle^{f_{v}} & =\left[\left\langle\gamma_{\mathbf{C}^{\times}}(\boldsymbol{z}), t\right\rangle_{\Sigma, v}\right]^{f_{v}} \\
& =\mathfrak{H}_{v}\left(\gamma_{\mathbf{C}^{\times}}(\boldsymbol{z}), t\right) .
\end{aligned}
$$

Or, pour $\boldsymbol{z} \in X(T)_{\boldsymbol{U}}^{G}$, on a d'après (5.19)

$$
\left(\chi_{\boldsymbol{z}}\right)_{v}(t)=\left\langle\boldsymbol{z}, \operatorname{deg}_{T, v}(t)\right\rangle^{f_{v}}
$$

d'où la relation (5.67).

Soit $t \in T\left(K_{v}\right)$. D'après le lemme 5.5 il existe un élément $\sigma \in \Sigma^{G_{v}}$ tel que $\operatorname{deg}_{T, L, v}(t)$ s'écrit

$$
\operatorname{deg}_{T, L, \mathcal{V}}(t)=\sum_{\beta \in \sigma(1) / G_{v}} n_{\beta} \tau_{\beta}
$$

où les $n_{\beta}$ sont des entiers strictement positifs. Pour tout élément $\varphi$ de $\operatorname{PL}(\Sigma)^{G}$ (identifié à $P_{\Sigma}^{G}$ ), on a alors

$$
f_{v}\langle\varphi, t\rangle=\frac{f_{v}}{e_{v}}\left\langle\varphi, \operatorname{deg}_{T, L, v}(t)\right\rangle_{\Sigma, v}=\frac{f_{v}}{e_{v}} \sum_{\beta \in \sigma(1) / G_{v}} n_{\beta} l_{\beta}\left\langle\varphi, D_{\beta}^{\vee}\right\rangle .
$$

Ainsi la condition $v$ vérifie l'hypothèse 4.8 entraîne qu'on a

$$
\forall \beta \in \sigma(1) / G_{v}, \quad \frac{f_{v} n_{\beta} l_{\beta}}{e_{v}} \in \mathbf{Z} .
$$

On a alors, d'après (5.72) et la définition (4.41) de $\mathfrak{H}_{v}$,

$$
\forall \boldsymbol{z} \in\left(P_{\Sigma}^{G}\right)_{\mathbf{C}^{\times}}, \quad \mathfrak{H}_{v}(\boldsymbol{z}, t)=\left\langle\boldsymbol{z}, \sum_{\beta \in \sigma(1) / G_{v}} \frac{f_{v} n_{\beta} l_{\beta}}{e_{v}} D_{\beta}^{\vee}\right\rangle
$$

ce qui montre, d'après le lemme 5.15 , que $\mathfrak{H}_{v}(., t)$ s'étend en un monôme sur $\mathbf{C}^{\Sigma(1) / G}$. Comme les $n_{\beta}$ sont strictement positifs, ce monôme est une constante si et seulement si $\sigma(1) / G_{v}$ est vide, c'est-à-dire si et seulement si $\operatorname{deg}_{T, L, \mathcal{V}}(t)$ est nul, soit encore si et seulement si $t \in T\left(\mathcal{O}_{v}\right)$.

La relation

$$
\forall \boldsymbol{z} \in X(T)_{\mathbf{C}^{\times}}^{G}, \quad \mathfrak{H}_{v}\left(\gamma_{\mathbf{C}^{\times}}(\boldsymbol{z}), t\right)=\left\langle\boldsymbol{z}, f_{v} \operatorname{deg}_{T, v}(t)\right\rangle,
$$

le fait que $\operatorname{deg}_{T, v}(t)$ soit dans $\mathcal{D}_{T}$, et le lemme 5.18 montrent que $\mathfrak{H}_{v}(., t)$ est un monôme $\mathcal{D}_{T}$-compatible. 


\section{Corollaire 5.22}

Soit $v$ une place de $K$ vérifiant l'hypothèse 4.8. Pour tout $t \in T\left(K_{v}\right)$, on a

$$
\forall \boldsymbol{z} \in X(T)_{\boldsymbol{U}}^{G}, \quad \forall \boldsymbol{s} \in \operatorname{PL}(\Sigma)_{\boldsymbol{C}}^{G}, \quad H_{v}(\boldsymbol{s}, t)\left(\chi_{\boldsymbol{z}}\right)_{v}(t)=\mathfrak{H}_{v}\left(\gamma_{\boldsymbol{U}}(\boldsymbol{z}) q^{\boldsymbol{s}}, t\right)
$$

\section{Proposition 5.23}

Soit $v$ une place de $K$ vérifiant l'hypothèse 4.8 et $\chi$ un élément de $\left(T\left(\boldsymbol{A}_{K}\right) / \boldsymbol{K}(T)\right)^{*}$. La série formelle

$$
\mathfrak{f}_{v}(\chi, .)=\left(\int_{T\left(\mathcal{O}_{v}\right)} d \mu_{v}\right) \sum_{t \in T\left(K_{v}\right) / T\left(\mathcal{O}_{v}\right)} \chi_{v}(t) \mathfrak{H}_{v}(., t)
$$

a un rayon de convergence supérieur à 1 , est $\mathcal{D}_{T}$-compatible, et vérifie

$$
\forall \boldsymbol{s} \in \mathcal{T}\left(\boldsymbol{R}_{>0}^{\Sigma(1) / G}\right), \quad \mathfrak{f}_{v}\left(\chi, q^{-s}\right)=f_{v}(\chi, s)
$$

Démonstration: Comme le morphisme $\operatorname{deg}_{T, L, \mathcal{V}}$ induit une injection de $T\left(K_{v}\right) / T\left(\mathcal{O}_{v}\right)$ dans $\left(X(T)^{\vee}\right)^{G_{v}}, T\left(K_{v}\right) / T\left(\mathcal{O}_{v}\right)$ est un groupe abélien de type fini, donc un monoïde commutatif de type fini. Par ailleurs, pour tout $t \in$ $T\left(K_{v}\right) / T\left(\mathcal{O}_{v}\right)$ et tout $n \in \mathbf{N}$, on a

$$
\mathfrak{H}_{v}\left(., t^{n}\right)=\mathfrak{H}_{v}(., t)^{n}
$$

et d'après le lemme $5.21, \mathfrak{H}_{v}(., t)$ est une constante si et seulement si $t=0$. Ce qui précède montre que pour tout entier $d$ il n'existe qu'un nombre fini de $t \in T\left(K_{v}\right) / T\left(\mathcal{O}_{v}\right)$ tel que le monôme $\mathfrak{H}_{v}(., t)$ soit de degré total majoré par $d$. Ainsi $\mathfrak{f}_{v}(\chi,$.$) est bien une série formelle.$

Le fait que le rayon de convergence soit supérieur à 1 et la relation (5.78) viennent de la remarque 5.8 et de (4.43). La $\mathcal{D}_{T}$-compatibilité découle aussitôt de la $\mathcal{D}_{T}$-compatibilité des $\mathfrak{H}_{v}(., t)$ (lemme 5.21).

\section{Lemme 5.24}

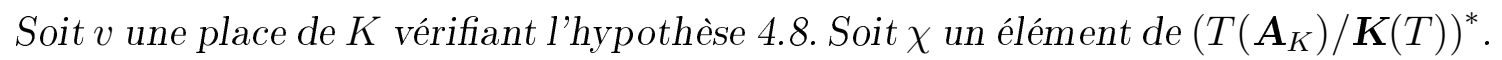
Alors on a

$$
\begin{aligned}
\forall \boldsymbol{z} \in X(T)_{\boldsymbol{U}}^{G}, \quad \forall \boldsymbol{s} \in \mathcal{T}\left(\boldsymbol{R}_{>0}^{\Sigma(1) / G}\right), \\
f_{v}\left(\chi \cdot \chi_{\boldsymbol{z}}, \boldsymbol{s}\right)=\mathfrak{f}_{v}\left(\chi, \gamma_{\boldsymbol{U}}(\boldsymbol{z}) q^{-\boldsymbol{s}}\right) .
\end{aligned}
$$


Démonstration: $\operatorname{Par}(5.78)$, le membre de gauche de (5.80) vaut $\mathfrak{f}_{v}\left(\chi \cdot \chi_{\boldsymbol{z}}, q^{-\boldsymbol{s}}\right)$, et on a d'après (5.77) et (5.67)

$$
\begin{aligned}
\mathfrak{f}_{v}\left(\chi \cdot \chi_{\boldsymbol{z}}, q^{-\boldsymbol{s}}\right) & =\left(\int_{T\left(\mathcal{O}_{v}\right)} d \mu_{v}\right) \sum_{t \in T\left(K_{v}\right) / T\left(\mathcal{O}_{v}\right)} \chi_{v}(t)\left(\chi_{\boldsymbol{z}}\right)_{v}(t) \mathfrak{h}_{v}\left(q^{-\boldsymbol{s}}, t\right) \\
& =\left(\int_{T\left(\mathcal{O}_{v}\right)} d \mu_{v}\right) \sum_{t \in T\left(K_{v}\right) / T\left(\mathcal{O}_{v}\right)} \chi_{v}(t) \mathfrak{h}_{v}\left(\gamma_{\boldsymbol{U}}(\boldsymbol{z}) q^{-\boldsymbol{s}}, t\right) \\
& =\mathfrak{f}_{v}\left(\chi, \gamma_{\boldsymbol{U}}(\boldsymbol{z}) q^{-\boldsymbol{s}}\right)
\end{aligned}
$$

On considère désormais $S$ un ensemble fini de places de $K$ contenant toutes les places ramifiées dans $L$, et tel que toutes les places de $S$ vérifient l'hypothèse 4.8. On note

$$
\mathbf{K}(T)^{S}=\prod_{v \in S} T\left(\mathcal{O}_{v}\right)
$$

et

$$
C_{S}=\int_{T(K)}{ }_{S}\left(\underset{\mathbf{K}(T)^{S}}{\otimes} d \mu_{v \in S}\right)
$$

Pour $t \in T\left(\mathbf{A}_{K}\right)$, on note $\tilde{H}_{S}(., t)$ le monôme défini par

$$
\mathfrak{H}_{S}(., t)=\prod_{v \in S} \mathfrak{H}_{v}\left(., t_{v}\right) .
$$

\section{Lemme 5.25}

Soit $S$ un ensemble fini de places de $K$ contenant toutes les places ramifiées dans $L$, et tel que toutes les places de $S$ vérifient l'hypothèse 4.8. La série formelle $\mathfrak{f}_{S}$ définie par

$$
\mathfrak{f}_{S}=C_{S} \sum_{t \in \overline{T(K)} S /\left(\frac{T(K)}{T\left(\cap \boldsymbol{K}(T)^{S}\right)}\right.} \mathfrak{H}_{S}(., t)
$$

a un rayon de convergence supérieur à 1 , est $\mathcal{D}_{T}^{0}$-compatible et vérifie, pour tout $\boldsymbol{s} \in \mathcal{T}\left(\boldsymbol{R}_{>0}^{\Sigma(1) / G}\right)$

$$
\int_{\frac{T(K)}{S}} H(-\boldsymbol{s}, t) \chi_{\boldsymbol{z}}(t)\left(\otimes_{v \in S} d \mu_{v}\right)(t)=\mathfrak{f}_{S}\left(\gamma_{\boldsymbol{U}}(\boldsymbol{z}) q^{-\boldsymbol{s}}\right)
$$


Démonstration: Comme $\overline{T(K)}{ }^{S} /\left(\overline{T(K)}^{S} \cap \mathbf{K}(T)^{S}\right)$ s'injecte dans $\prod_{v \in S} T\left(K_{v}\right) / T\left(\mathcal{O}_{v}\right)$, un argument similaire à celui du début de la proposition 5.23 montre que $\mathfrak{f}_{S}$ est bien une série formelle.

Pour $s \in \mathcal{T}\left(\mathbf{R}_{>0}^{\Sigma(1) / G}\right)$, la convergence de la série

$$
\sum_{t \in \overline{T(K)} S /\left(\overline{T(K)} S \cap \mathbf{K}(T)^{S}\right)} \prod_{v \in S} \mathfrak{H}_{v}\left(q^{-\Re(s)}, t_{v}\right)
$$

se montre en injectant $\overline{T(K)}^{S} /\left(\overline{T(K)}^{S} \cap \mathbf{K}(T)^{S}\right)$ dans $\prod_{v \in S} T\left(K_{v}\right) / T\left(\mathcal{O}_{v}\right)$, ce qui permet de majorer (5.89) par

$$
\prod_{v \in S} \sum_{t \in T\left(K_{v}\right) / T\left(\mathcal{O}_{v}\right)} H_{v}\left(-\Re(s), t_{v}\right)
$$

qui est un produit de séries convergentes (cf. remarque 5.8).

On a ainsi, toujours pour $\boldsymbol{s} \in \mathcal{T}\left(\mathbf{R}_{>0}^{\Sigma(1) / G}\right)$

$$
\begin{aligned}
& \int_{\frac{T(K)}{T(S}} H(-s, t) \chi_{\boldsymbol{z}}(t)\left(\underset{v \in S}{\otimes} d \mu_{v}\right)(t) \\
& =C_{S} \sum_{t \in \overline{T(K)} S /\left(\frac{T(K)}{S} \cap \mathbf{K}(T)^{S}\right)} H(-\boldsymbol{s}, t) \chi_{\boldsymbol{z}}(t) \\
& =C_{S} \sum_{t \in \overline{T(K)} S /\left(\frac{T(K)}{S} \cap \mathbf{K}(T)^{S}\right)} H(-\boldsymbol{s}, t)\left\langle\boldsymbol{z}, \operatorname{deg}_{T}(t)\right\rangle \\
& =C_{S} \sum_{t \in \overline{T(K)} S /\left(\overline{T(K)} S_{\cap} \mathbf{K}_{(T)^{S}}\right)} \prod_{v \in S} \mathfrak{H}_{v}\left(\gamma_{\boldsymbol{U}}(\boldsymbol{z}) q^{-\boldsymbol{s}}, t_{v}\right)
\end{aligned}
$$

Pour terminer la démonstration du lemme, il suffit de montrer que pour $t \in \overline{T(K)}^{S}$, le monôme $\mathfrak{H}_{S}(., t)$ est $\mathcal{D}_{T}^{0}$ compatible. Soit donc $t=\left(t_{v}\right)_{v \in S} \in$ $\overline{T(K)}^{S}$. D'après la proposition 3.32, il existe $u \in T(K)$ et $t^{\prime} \in T\left(\mathbf{A}_{K}\right)$ un élément de l'image de $T_{P_{\Sigma}}\left(\mathbf{A}_{K}\right)$ par $\gamma_{\mathbf{A}_{K}}$ tels que $t=u t^{\prime}$. Comme $\operatorname{deg}_{T}$ est trivial sur $T(K)$, on $\operatorname{a~deg}_{T}(t)=\operatorname{deg}_{T}\left(t^{\prime}\right)$. Comme $t^{\prime}$ est dans $\gamma_{\mathbf{A}_{K}}\left(T_{P_{\Sigma}}\left(\mathbf{A}_{K}\right)\right)$, $\operatorname{deg}_{T}\left(t^{\prime}\right)$ est dans $\mathcal{D}_{T}^{0}$. Ainsi $\operatorname{deg}_{T}(t)$ est dans $\mathcal{D}_{T}^{0}$. Comme on a, pour tout $\boldsymbol{z} \in X(T)_{\mathbf{C}}^{G}$,

$$
\mathfrak{H}_{S}(\boldsymbol{z}, t)=\left\langle\boldsymbol{z}, \operatorname{deg}_{T}(t)\right\rangle
$$

on voit, d'après le lemme 5.18 , que $\mathfrak{H}_{S}(., t)$ est $\mathcal{D}_{T}^{0}$-compatible. 


\subsubsection{Cas des places non ramifiées}

Soit $v$ une place finie de $K, \mathcal{V}$ une place de $L$ divisant $v$ et $G_{v}$ son groupe de décomposition. Les auteurs de [BaTs1] définissent ${ }^{5}$ alors un polynôme à coefficients entiers $Q_{\Sigma, v}$ en les $\left[\Sigma(1) / G_{v}\right]$ indéterminées $\left(X_{\beta}\right)_{\beta \in \Sigma(1) / G_{v}}$ par la formule

$$
\left(\int_{T\left(\mathcal{O}_{v}\right)} d \mu_{v}\right) \sum_{\sigma \in \Sigma^{G_{v}}} \prod_{\beta \in \sigma(1) / G_{v}} \frac{X_{\beta}}{1-X_{\beta}}=\frac{Q_{\Sigma, v}\left(X_{\beta}\right)}{\prod_{\beta \in \Sigma(1) / G_{v}}\left(1-X_{\beta}\right)} .
$$

Un résultat facile mais important pour les résultats de convergence et de prolongement de la fonction zêta des hauteurs est (cf. [BaTs1, Proposition $2.2 .3])$

\section{Proposition 5.26 (Batyrev, Tschinkel)}

Le polynôme $Q_{\Sigma, v}\left(X_{\beta}^{l_{\beta}}\right)-1$ ne contient que des monômes de degré total supérieur ou égal à 2 .

Désormais nous fixons un sous-ensemble fini $S$ de $\mathcal{P}_{K}$ contenant les places archimédiennes, et les places ramifiées dans $L / K$. Le résultat suivant ([BaTs1, Theorem 2.2.6]) donne alors une expression explicite des transformées de Fourier locales aux places $v \notin S$.

Théorème 5.27 (Batyrev, Tschinkel)

Soit $v \notin S$ une place de $K$. Soit $\boldsymbol{s} \in \mathcal{T}\left(\boldsymbol{R}_{>0}^{\Sigma(1) / G}\right)$. On a pour tout $\chi \in$ $\left(T\left(\boldsymbol{A}_{K}\right) / \boldsymbol{K}(T)\right)^{*}$

$$
\begin{aligned}
& \int_{T\left(K_{v}\right)} H_{v}(-s, t) \chi_{v}(t) d \mu_{v}(t) \\
& \quad=\left(\prod_{\beta \in \Sigma(1) / G_{v}} \frac{1}{1-\chi_{\beta}\left(\pi_{w_{\beta}}\right) q_{v}^{-l_{\beta} s_{\beta}}}\right) Q_{\Sigma, v}\left(\chi_{\beta}\left(\pi_{w_{\beta}}\right) q_{v}^{-l_{\beta} s_{\beta}}\right)_{\beta \in \Sigma(1) / G_{v}} .
\end{aligned}
$$

Démonstration : Nous rappelons la preuve de ce théorème, donnée dans [BaTs1]. À l'aide du morphisme $\operatorname{deg}_{T, L, \mathcal{V}}$, on identifie $T\left(K_{v}\right) / T\left(\mathcal{O}_{v}\right) \grave{a ̀ ~}\left(X(T)^{\vee}\right)^{G_{v}}$

\footnotetext{
${ }^{5}$ la définition utilisée dans $[\mathrm{BaTs} 1]$ est en fait légèrement différente car on y utilise le polynôme $Q_{\Sigma, v}\left(X_{\beta}^{l_{\beta}}\right)$, ceci étant on obtient bien la même formule pour la transformée de Fourier locale.
} 
(cf. proposition 3.27). Comme les fonctions $H_{v}(-\boldsymbol{s},$.$) et \chi_{v}$ sont $T\left(\mathcal{O}_{v}\right)$ invariante, elles induisent des fonctions sur $\left(X(T)^{\vee}\right)^{G_{v}}$, qui seront notées de façon identique. On a alors

$$
\int_{T\left(K_{v}\right)} H_{v}(-\boldsymbol{s}, t) \chi_{v}(t) d \mu_{v}(t)=\int_{T\left(\mathcal{O}_{v}\right)} d \mu_{v} \sum_{t \in T\left(K_{v}\right) / T\left(\mathcal{O}_{v}\right)} H_{v}(-\boldsymbol{s}, t) \chi_{v}(t)
$$

Le lemme 5.5 permet alors d'écrire

$$
\begin{aligned}
& \sum_{t \in T\left(K_{v}\right) / T\left(\mathcal{O}_{v}\right)} H_{v}(-s, t) \chi_{v}(t) \\
= & \sum_{\sigma \in \Sigma^{G_{v}}} \sum_{t \in \operatorname{intrel}(\sigma) \cap\left(T(T)^{\vee}\right)^{G_{v}}} H_{v}(-\boldsymbol{s}, t) \chi_{v}(t) \\
= & \sum_{\sigma \in \Sigma^{G}} \sum_{\left(n_{\beta}\right) \in \mathbf{Z}_{>0}^{\sigma(1) / G_{v}}} H_{v}\left(-s, \sum n_{\beta} \tau_{\beta}\right) \prod \chi_{v}\left(\tau_{\beta}\right)^{n_{\beta}} .
\end{aligned}
$$

Mais on a

$$
\begin{aligned}
& \sum_{\sigma \in \Sigma^{G v}} \sum_{\left(n_{\beta}\right) \in \mathbf{Z}_{>0}^{\sigma(1) / G_{v}}} H_{v}\left(-\boldsymbol{s}, \sum n_{\beta} \tau_{\beta}\right) \prod \chi_{v}\left(\tau_{\beta}\right)^{n_{\beta}} \\
= & \sum_{\sigma \in \Sigma^{G_{v}}} \sum_{\left(n_{\beta}\right) \in \mathbf{Z}_{>0}^{\sigma(1) / G_{v}}} H_{v}\left(-\boldsymbol{s}, \sum n_{\beta} \tau_{\beta}\right) \prod \chi_{\beta}\left(\pi_{w_{\beta}}\right)^{n_{\beta}} \\
= & \sum_{\sigma \in \Sigma^{G} v} \prod_{\beta \in \sigma(1) / G_{v}} \sum_{n_{\beta} \in \mathbf{Z}_{>0}} q_{v}^{-n_{\beta} l_{\beta} s_{\beta}} \chi_{\beta}\left(\pi_{w_{\beta}}\right)^{n_{\beta}} \\
= & \sum_{\sigma \in \Sigma^{G} v} \prod_{\beta \in \sigma(1) / G_{v}} \frac{\chi_{\beta}\left(\pi_{w_{\beta}}\right) q_{v}^{-l_{\beta} s_{\beta}}}{1-\chi_{\beta}\left(\pi_{w_{\beta}}\right) q_{v}^{-l_{\beta} s_{\beta}}} .
\end{aligned}
$$

d'où le résultat par définition de $Q_{\Sigma, v}$.

L'égalité (5.103) vient du fait que si $v$ n'est pas ramifiée dans $L$, d'après le lemme 3.26 , on a

$$
\operatorname{deg}_{T, L, \nu}\left[\gamma_{v} \circ i_{\beta, v}\left(\pi_{w_{\beta}}\right)\right]=\tau_{\beta} .
$$

L'égalité (5.104) vient de ce que par la définition (4.20) de la hauteur locale et le fait que $v$ est non ramifiée on a

$$
\begin{aligned}
H_{v}\left(-s, \sum_{\beta \in \sigma(1) / G_{v}} n_{\beta} \tau_{\beta}\right) & =\exp \left(\log \left(q_{v}\right) \sum_{\beta \in \sigma(1) / G_{v}} n_{\beta}\left\langle\varphi, \tau_{\beta}\right\rangle_{\Sigma, v}\right) \\
& =\exp \left(\log \left(q_{v}\right) \sum_{\beta \in \sigma(1) / G_{v}} n_{\beta} l_{\beta} s_{\beta}\right)
\end{aligned}
$$




\section{Lemme 5.28}

1. Pour tout $\alpha \in \Sigma(1) / G$, on a

$$
\prod_{\beta \in \alpha / G_{v}} \frac{1}{1-\chi_{\beta}\left(\pi_{w_{\beta}}\right) q_{v}^{-l_{\beta} s_{\beta}}}=\prod_{\substack{w \in \mathcal{P}_{K_{\alpha}} \\ w \mid v}} \frac{1}{1-\chi_{\alpha}\left(\pi_{w}\right) q_{w}^{-s_{\alpha}}} .
$$

En particulier, dans le cas fonctionnel, on a

$$
\prod_{\beta \in \alpha / G_{v}} \frac{1}{1-\chi_{\beta}\left(\pi_{w_{\beta}}\right) q_{v}^{-l_{\beta} s_{\beta}}}=\prod_{\substack{w \in \mathcal{P}_{K_{\alpha}} \\ w \mid v}} \frac{1}{1-\chi_{\alpha}\left(\pi_{w}\right) q^{-f_{w} d_{\alpha} s_{\alpha}}} .
$$

2. Dans le cas arithmétique, pour $\chi \in\left(T\left(\boldsymbol{A}_{K}\right) / \boldsymbol{K}(T)\right)^{*}$ et $y \in X(T)_{\boldsymbol{R}}^{G}$, on a

$$
\left(\chi \cdot \chi_{y}\right)_{\alpha}\left(\pi_{w}\right) q_{w}^{-s_{\alpha}}=\chi_{\alpha}\left(\pi_{w}\right) q_{w}^{-s_{\alpha}+i\left\langle y, \rho_{\alpha}\right\rangle} .
$$

3. Dans le cas fonctionnel, pour $\chi \in\left(T\left(\boldsymbol{A}_{K}\right) / \mathbf{K}(T)\right)^{*}$ et $\boldsymbol{z} \in X(T)_{U}^{G}$, on a

$$
\left(\chi \cdot \chi_{\boldsymbol{z}}\right)_{\alpha}\left(\pi_{w}\right) q^{-f_{w} d_{\alpha} s_{\alpha}}=\chi_{\alpha}\left(\pi_{w}\right)\left\langle\boldsymbol{z}, d_{\alpha} \rho_{\alpha}\right\rangle q^{-f_{w} d_{\alpha} s_{\alpha}} .
$$

Démonstration: Le point 1 provient des formules (5.11) et (5.13). Le point 2 découle du lemme 5.2 et le point 3 du lemme 5.3.

Le résultat suivant sera utile lors du calcul du terme principal de la fonction zêta des hauteurs.

\section{Lemme 5.29}

Soit $v \notin S$. Alors pour tout $s \in \mathcal{T}\left(\boldsymbol{R}_{>0}\right)$, on a

$$
\int_{T\left(K_{v}\right)} H_{v}\left(-s \varphi_{0}, t\right) d \mu_{v}(t)=L_{v}\left(s, P_{\Sigma}\right) Q_{\Sigma, v}\left(q_{v}^{-l_{\beta} s}\right)_{\beta \in \Sigma(1) / G_{v}} .
$$

Démonstration: Ceci découle aussitôt d'une part du théorème 5.27 et du lemme 5.28, d'autres part des lemmes 2.5, 2.4 et de la décomposition (4.9).

\subsection{Propriétés analytiques de la transformée de Fourier globale}

On va déduire des résultats de la section 5.4 que pour tout élément $\boldsymbol{s}$ de $\mathcal{T}\left(\mathbf{R}_{>1}^{\Sigma(1) / G}\right)$, la fonction $H(-s,$.$) est intégrable sur T\left(\mathbf{A}_{K}\right)$, ainsi que des 
renseignements sur le comportement analytiques des transformées de Fourier globales.

Pour $\alpha \in \Sigma(1) / G$, nous notons $S_{\alpha}$ les places de $K_{\alpha}$ au-dessus des places de $S$.

\section{Lemme 5.30}

Pour tout $\boldsymbol{s}$ dans $\mathcal{T}\left(\boldsymbol{R}_{>\frac{1}{2}}^{\Sigma(1) / G}\right)$ le produit eulérien

$$
\prod_{v \notin S} Q_{\Sigma, v}\left(q_{v}^{-l_{\beta} s_{\beta}}\right)_{\beta \in \Sigma(1) / G_{v}}
$$

est absolument convergent.

Démonstration : Ceci découle aussitôt de la proposition 5.26.

\section{Lemme 5.31}

Pour tout $\boldsymbol{s} \in \mathcal{T}\left(\boldsymbol{R}_{>1}^{\Sigma(1) / G}\right), H(-\boldsymbol{s},$.$) est intégrable sur T\left(\boldsymbol{A}_{K}\right)$.

Démonstration : On a pour tout $s \in \mathbf{C}^{\Sigma(1) / G}$

$$
\int_{T\left(\mathbf{A}_{K}\right)}|H(-s, t)| \omega_{T}(t)=\int_{T\left(\mathbf{A}_{K}\right)} H(-\Re(\boldsymbol{s}), x) \omega_{T}(t) .
$$

D'après la définition (3.6) de $\omega_{T}$ cette dernière expression vaut

$$
c_{K, \operatorname{dim}(T)} \prod_{v \in \mathcal{P}_{K}} \int_{T\left(K_{v}\right)} H_{v}(-\Re(\boldsymbol{s}), t) d \mu_{v}(t) .
$$

D'après le théorème 5.27 et le lemme 5.28 , on a donc

$$
\begin{aligned}
\int_{T\left(\mathbf{A}_{K}\right)}|H(-s, t)| \omega_{T}(t) & \left(\prod_{\alpha \in \Sigma(1) / G} \zeta_{K_{\alpha}}\left(\Re\left(s_{\alpha}\right)\right)\right)\left(\prod_{\alpha \in \Sigma(1) / G} \prod_{w \in S_{\alpha}}\left(1-q_{w}^{-\Re\left(s_{\alpha}\right)}\right)\right) \\
= & c_{K, \operatorname{dim}(T)}\left(q_{v}^{-l_{\beta} \Re\left(s_{\beta}\right)}\right)_{\beta \in \Sigma(1) / G_{v}} \\
& \prod_{v \in S} \int_{v \notin S} H_{v, v}(-\Re(\boldsymbol{s}), t) d \mu_{v}(t)
\end{aligned}
$$

D'après la proposition 2.1, le lemme 5.30, le point 1 de la proposition 5.7 et, dans le cas arithmétique, le point 1 de la proposition 5.12, cette dernière expression est finie dès que $\Re(\boldsymbol{s}) \in \mathbf{R}_{>1}^{\Sigma(1) / G}$ d'où l'intégrabilité de $H(-\boldsymbol{s},$. pour $\boldsymbol{s} \in \mathcal{T}\left(\mathbf{R}_{>1}^{\Sigma(1) / G}\right)$. 


\subsubsection{Cas arithmétique}

\section{Lemme 5.32}

Soit $\chi \in\left(T\left(\boldsymbol{A}_{K}\right) / \boldsymbol{K}(T)\right)^{*}$. La fonction

$$
\begin{aligned}
& s \mapsto c_{K, \operatorname{dim}(T)} \prod_{v \notin S} Q_{\Sigma, v}\left(\chi_{\beta}\left(\pi_{w_{\beta}}\right) q_{v}^{-l_{\beta} s_{\beta}}\right)_{\beta \in \Sigma(1) / G_{v}} \\
& \prod_{v \in S \backslash \mathcal{P}_{K, \infty}} f_{v}(\chi, s) \prod_{\alpha \in \Sigma(1) / G} \prod_{w \in S_{\alpha}}\left(1-\chi_{\alpha}\left(\pi_{w}\right) q_{w}^{-s_{\alpha}}\right)
\end{aligned}
$$

est holomorphe sur $\mathcal{T}\left(\boldsymbol{R}_{>\frac{1}{2}}^{\Sigma(1) / G}\right)$. On la note $f(\chi,$.$) .$

Démonstration: Ceci découle du lemme 5.30 et du point 2 de la proposition 5.7.

Rappelons qu'on a également noté

$$
f_{\infty}(\chi, .)=\prod_{v \in \mathcal{P}_{K, \infty}} f_{v}(\chi, .)
$$

\section{Lemme 5.33}

$f_{\infty}(\chi,$.$) est holomorphe sur \mathcal{T}\left(\boldsymbol{R}_{>0}^{\Sigma(1) / G}\right)$.

Démonstration : D'après le point 2 de la proposition 5.12, pour tout $v \in \mathcal{P}_{K, \infty}$ la fonction $f_{v}(\chi,$.$) est holomorphe sur \mathcal{T}\left(\mathbf{R}_{>0}^{\Sigma(1) / G}\right)$.

\section{Lemme $\mathbf{5 . 3 4}$}

Soit $\chi \in\left(T\left(\boldsymbol{A}_{K}\right) / \boldsymbol{K}(T) \cdot T(K)\right)^{*}$. Pour tout $\boldsymbol{s} \in \mathcal{T}\left(\boldsymbol{R}_{>1}^{\Sigma(1) / G}\right)$, et tout $y \in$ $X(T)_{\mathbf{R}}^{G}$, on a

$$
\begin{aligned}
& \mathcal{F} H\left(\chi \cdot \chi_{y},-s\right) \\
& =\left(\prod_{\alpha \in \Sigma(1) / G} L_{K_{\alpha}}\left(s_{\alpha}-i\left\langle y, \rho_{\alpha}\right\rangle, \chi_{\alpha}\right)\right) f\left(\chi, s-i \gamma_{\boldsymbol{R}}(y)\right) f_{\infty}\left(\chi, s-i \gamma_{\boldsymbol{R}}(y)\right) .
\end{aligned}
$$

Démonstration : D'après la définition (3.6) de $\omega_{T}$, on a

$$
\int_{T\left(\mathbf{A}_{K}\right)}\left(\chi \chi_{y}\right)(t) H(-\boldsymbol{s}, t) \omega_{T}(t)=c_{K, \operatorname{dim}(T)} \prod_{v \in \mathcal{P}_{K}} \int_{T\left(K_{v}\right)}\left(\chi \chi_{y}\right)_{v}(t) H_{v}(-\boldsymbol{s}, t) d \mu_{v}(t) .
$$


D'après le théorème 5.27 , le lemme 5.28 et le corollaire 5.11 , le membre de gauche de (5.8) est donc égal à

$$
\begin{gathered}
c_{K, \operatorname{dim}(T)} \prod_{\alpha \in \Sigma(1) / G} L_{K_{\alpha}}\left(\chi_{\alpha}, s_{\alpha}-i\left\langle y, \rho_{\alpha}\right\rangle\right) \\
\times \prod_{\alpha \in \Sigma(1) / G} \prod_{w \in S_{\alpha}}\left(1-\chi_{\alpha}\left(\pi_{w}\right) q_{w}^{-s_{\alpha}+i\left\langle y, \rho_{\alpha}\right\rangle}\right) \\
\times \prod_{v \notin S} Q_{\Sigma, v}\left(\chi_{\beta}\left(\pi_{w_{\beta}}\right) q_{v}^{-l_{\beta}\left(s_{\beta}-i\left\langle y, \rho_{\beta}\right\rangle\right)}\right)_{\beta \in \Sigma(1) / G_{v}} \\
\times \prod_{v \in S} f_{v}\left(\chi, s-i \gamma_{\mathbf{R}}(y)\right)
\end{gathered}
$$

d'où le résultat.

\section{Lemme 5.35}

Soit $\boldsymbol{K}$ un compact de $\boldsymbol{R}_{>\frac{1}{2}}^{\Sigma(1) / G}$. Il existe une constante $C>0$ telle qu'on ait, pour tout $\chi \in\left(T\left(\boldsymbol{A}_{K}\right) / \mathbf{K}(T)\right)^{*}$ et tout $\boldsymbol{s} \in \mathcal{T}(\boldsymbol{K})$ la majoration

$$
|f(\chi, s)| \leqslant C \text {. }
$$

Démonstration : Ceci découle immédiatement du point 3 de la proposition 5.7, de la majoration

$$
\left|Q_{\Sigma, v}\left(\chi_{\beta}\left(\pi_{w_{\beta}}\right) q_{v}^{-l_{\beta} s_{\beta}}\right)_{\beta \in \Sigma(1) / G_{v}}\right| \leqslant Q_{\Sigma, v}\left(q_{v}^{-l_{\beta} \Re\left(s_{\beta}\right)}\right)_{\beta \in \Sigma(1) / G_{v}}
$$

et du fait que

$$
\boldsymbol{s} \mapsto \prod_{v \notin S} Q_{\Sigma, v}\left(q_{v}^{-l_{\beta} s_{\beta}}\right)_{\beta \in \Sigma(1) / G_{v}}
$$

est holomorphe sur $\mathbf{R}_{>\frac{1}{2}}^{\Sigma(1) / G}$.

\subsubsection{Cas fonctionnel}

\section{Lemme 5.36}

Soit $\chi \in\left(T\left(\boldsymbol{A}_{K}\right) / \mathbf{K}(T)\right)^{*}$. La série formelle $\mathfrak{Q}(\chi,$.$) définie par$

$$
\mathfrak{Q}\left(\chi,\left(z_{\alpha}\right)\right) \stackrel{\text { déf }}{=} c_{K, \operatorname{dim}(T)} \prod_{v \notin S} Q_{\Sigma, v}\left(\chi_{\beta}\left(\pi_{w_{\beta}}\right) z_{\beta}^{f_{w_{\beta}} d_{\beta}}\right)
$$

a un rayon de convergence supérieur à $q^{-\frac{1}{2}}$, est $\mathcal{D}_{T}^{0}$-compatible et vérifie pour tout $\boldsymbol{s} \in \mathcal{T}\left(\boldsymbol{R}_{>\frac{1}{2}}^{\Sigma(1) / G}\right)$

$$
\mathfrak{Q}\left(\chi, q^{-s}\right)=c_{K, \operatorname{dim}(T)} \prod_{v \notin S} Q_{\Sigma, v}\left(\chi_{\beta}\left(\pi_{w_{\beta}}\right) q_{v}^{-l_{\beta} s_{\beta}}\right) .
$$


Démonstration : $\quad$ Pour $v \notin S, \beta \in \Sigma(1) / G_{v}$ et $s \in \mathbf{C}$, on a

$$
q_{v}^{-l_{\beta} s}=\left(q^{-s}\right)^{f_{w_{\beta}} d_{\beta}} .
$$

Ceci joint au lemme 5.30 montre aussitôt que le rayon de convergence de $\mathfrak{Q}(\chi,$.$) est supérieur à q^{-\frac{1}{2}}$, ainsi que la relation (5.14).

La $\mathcal{D}_{T}^{0}$-compatibilité provient du lemme 5.19.

\section{Lemme 5.37}

On suppose que toutes les places de $K$ vérifient l'hypothèse 4.8. Soit $\chi \in$ $\left(T\left(\boldsymbol{A}_{K}\right) / \mathbf{K}(T)\right)^{*}$. La série formelle

$$
\mathfrak{f}(\chi, \boldsymbol{z}) \stackrel{\text { déf }}{=} \mathfrak{Q}(\chi, \boldsymbol{z}) \prod_{v \in S} \mathfrak{f}_{v}(\chi, \boldsymbol{z}) \prod_{\alpha \in \Sigma(1) / G} \prod_{w \in S_{\alpha}}\left(1-\chi_{\alpha}\left(\pi_{w}\right) z_{\alpha}^{f_{w} d_{\alpha}}\right)
$$

a un rayon de convergence supérieur à $q^{-\frac{1}{2}}$ et est $\mathcal{D}_{T}$-compatible.

Démonstration: Ceci découle du théorème 5.27 et les lemmes 5.28 et 5.24 .

\section{Lemme 5.38}

On suppose que toutes les places de $K$ vérifient l'hypothèse 4.8. Soit $\chi \in$ $\left(T\left(\boldsymbol{A}_{K}\right) / \boldsymbol{K}(T)\right)^{*}$. On a pour tout $\boldsymbol{s} \in \mathcal{T}\left(\boldsymbol{R}_{>1}^{\Sigma(1) / G}\right)$ et tout $\boldsymbol{z} \in X(T)_{\boldsymbol{U}}^{G}$

$$
\mathcal{F} H\left(\chi \cdot \chi_{\boldsymbol{z}},-s\right)=\left(\prod_{\alpha \in \Sigma(1) / G} \mathcal{L}_{K_{\alpha}}\left(\chi_{\alpha},\left\langle\boldsymbol{z}, d_{\alpha} \rho_{\alpha}\right\rangle q^{-d_{\alpha} s_{\alpha}}\right)\right) \mathfrak{f}\left(\chi, \gamma_{\boldsymbol{U}}(\boldsymbol{z}) q^{-\boldsymbol{s}}\right)
$$

Démonstration : D'après la définition (3.6) de $\omega_{T}$, on a

$$
\int_{T\left(\mathbf{A}_{K}\right)}\left(\chi \chi_{\boldsymbol{z}}\right)(t) H(-\boldsymbol{s}, t) \omega_{T}(t)=c_{K, \operatorname{dim}(T)} \prod_{v \in \mathcal{P}_{K}} \int_{T\left(K_{v}\right)}\left(\chi \chi_{\boldsymbol{z}}\right)_{v}(t) H_{v}(-\boldsymbol{s}, t) d \mu_{v}(t) \text {. }
$$

D'après le théorème 5.27 , le lemme 5.28 et le lemme 5.24 , le membre de gauche de (5.18) est donc égal à

$$
\begin{gathered}
c_{K, \operatorname{dim}(T)} \prod_{\alpha \in \Sigma(1) / G} \mathcal{L}_{K_{\alpha}}\left(\chi_{\alpha},\left\langle\boldsymbol{z}, d_{\alpha} \rho_{\alpha}\right\rangle q^{-d_{\alpha} s_{\alpha}}\right) \\
\times \prod_{\alpha \in \Sigma(1) / G} \prod_{w \in S_{\alpha}}\left(1-\chi_{\alpha}\left(\pi_{w}\right)\left\langle\boldsymbol{z}, d_{\alpha} \rho_{\alpha}\right\rangle q^{-f_{w} d_{\alpha} s_{\alpha}}\right) \\
\times \prod_{v \notin S} Q_{\Sigma, v}\left(\chi_{\beta}\left(\pi_{w_{\beta}}\right)\left\langle y, d_{\beta} \rho_{\beta}\right\rangle q^{-f_{w} d_{\beta} s_{\beta}}\right)_{\beta \in \Sigma(1) / G_{v}} \\
\times \prod_{v \in S} \mathfrak{f}_{v}\left(\chi, \gamma_{\boldsymbol{U}}(\boldsymbol{z}) q^{-\boldsymbol{s}}\right),
\end{gathered}
$$


d'où le résultat.

\section{Lemme 5.39}

On suppose que toutes les places de $K$ vérifient l'hypothèse 4.8. La série formelle

$$
\mathfrak{f}(\boldsymbol{z}) \stackrel{\text { déf }}{=} \mathfrak{Q}(\mathbf{1}, \boldsymbol{z}) \mathfrak{f}_{S}(\boldsymbol{z}) \prod_{\alpha \in \Sigma(1) / G} \prod_{w \in S_{\alpha}}\left(1-z_{\alpha}^{f_{w} d_{\alpha}}\right)
$$

a un rayon de convergence supérieur à $q^{-\frac{1}{2}}$ et est $\mathcal{D}_{T}^{0}$-compatible.

Démonstration : Ceci découle aussitôt du lemme 5.25 et du lemme 5.19.

\section{Lemme 5.40}

On suppose que toutes les places de $K$ vérifient l'hypothèse 4.8. On a, pour tout $\boldsymbol{s} \in \mathcal{T}\left(\boldsymbol{R}_{>1}^{\Sigma(1) / G}\right)$ et tout $\boldsymbol{z} \in X(T)_{U}^{G}$

$$
\begin{aligned}
\int_{\overline{T(K)} \cap T\left(\boldsymbol{A}_{K}\right)} & H(-\boldsymbol{s}, t) \chi_{\boldsymbol{z}}(t) \omega_{T}(t) \\
& =\left(\prod_{\alpha \in \Sigma(1) / G} Z_{K_{\alpha}}\left(\left\langle\boldsymbol{z}, d_{\alpha} \rho_{\alpha}\right\rangle q^{-d_{\alpha} s_{\alpha}}\right)\right) \mathfrak{f}\left(\gamma_{\boldsymbol{U}}(\boldsymbol{z}) q^{-\boldsymbol{s}}\right) .
\end{aligned}
$$


Démonstration : D'après le théorème 5.27 et le lemme 5.25 on a

$$
\begin{aligned}
& \int_{\overline{T(K)} \cap T\left(\mathbf{A}_{K}\right)} H(-\boldsymbol{s}, t) \chi_{\boldsymbol{z}}(t) \omega_{T}(t) \\
& =c_{K, \operatorname{dim}(T)}\left(\prod_{v \notin S} \int_{T\left(K_{v}\right)} H_{v}(-\boldsymbol{s}, t)\left(\chi_{\boldsymbol{z}}\right)_{v}(t) d \mu_{v}(t)\right) \\
& \times \int_{\frac{T(K)}{S}} H(-s, t)\left(\chi_{\boldsymbol{z}}\right)(t)\left(\underset{v \in S}{\otimes} d \mu_{v}\right)(t) \\
& =\prod_{\alpha \in \Sigma(1) / G} Z_{K_{\alpha}}\left(\left\langle\boldsymbol{z}, d_{\alpha} \rho_{\alpha}\right\rangle q^{-d_{\alpha} s_{\alpha}}\right) \\
& \times \prod_{\alpha \in \Sigma(1) / G} \prod_{w \in S_{\alpha}}\left(1-\boldsymbol{z}^{f_{w} d_{\alpha} \rho_{\alpha}} q^{-f_{w} d_{\alpha} s_{\alpha}}\right) \\
& \times \mathfrak{Q}\left(\mathbf{1}, \gamma_{\boldsymbol{U}}(\boldsymbol{z}) q^{-\boldsymbol{s}}\right) \mathfrak{f}_{S}\left(\gamma_{\boldsymbol{U}}(\boldsymbol{z}) q^{-\boldsymbol{s}}\right) \\
& =\left(\prod_{\alpha \in \Sigma(1) / G} Z_{K_{\alpha}}\left(\left\langle\boldsymbol{z}, d_{\alpha} \rho_{\alpha}\right\rangle q^{-d_{\alpha} s_{\alpha}}\right)\right) \mathfrak{f}\left(\gamma_{\boldsymbol{U}}(\boldsymbol{z}) q^{-\boldsymbol{s}}\right) \text {. }
\end{aligned}
$$

\subsection{L'expression intégrale de la fonction zêta des hau- teurs}

Notons $\gamma^{*}$ le morphisme de caractères

$$
\left(T\left(\mathbf{A}_{K}\right) / T(K)\right)^{*} \longrightarrow\left(T_{P_{\Sigma}}\left(\mathbf{A}_{K}\right) / T_{P_{\Sigma}}(K)\right)^{*},
$$

induit par la composition avec le morphisme $\gamma_{\mathbf{A}_{K}}$. Dans le cas fonctionnel comme dans le cas arithmétique, nous choisissons arbitrairement et une fois pour toutes un scindage du morphisme quotient

$$
T\left(\mathbf{A}_{K}\right) / T(K) \rightarrow T\left(\mathbf{A}_{K}\right) / T\left(\mathbf{A}_{K}\right)^{1} .
$$

Ce scindage induit par dualité un isomorphisme de $\left(T\left(\mathbf{A}_{K}\right)^{1} / T(K)\right)^{*}$ sur un facteur direct de $\left(T\left(\mathbf{A}_{K}\right) / T\left(\mathbf{A}_{K}\right)^{1}\right)^{*}$ dans $\left(T\left(\mathbf{A}_{K}\right) / T(K)\right)^{*}$, lequel facteur direct sera noté $\widetilde{\mathcal{U}_{T}}$. On note $\mathcal{U}_{T}$ l'image de $\left(T\left(\mathbf{A}_{K}\right)^{1} / \mathbf{K}(T) \cdot T(K)\right)^{*}$ dans $\widetilde{\mathcal{U}(T)}$ par cet isomorphisme. En d'autre termes, $\mathcal{U}_{T}$ est le sous-groupe de $\widetilde{\mathcal{U}_{T}}$ constitué des caractères qui sont triviaux sur $\mathbf{K}(T)$, et on a

$$
\left(T\left(\mathbf{A}_{K}\right) / \mathbf{K}(T) \cdot T(K)\right)^{*}=\left(T\left(\mathbf{A}_{K}\right) / T\left(\mathbf{A}_{K}\right)^{1}\right)^{*} \times \mathcal{U}_{T} .
$$




\section{Lemme 5.41}

1. Le noyau de $\gamma^{*}$ est isomorphe à $A(T)^{*}$. En particulier $\operatorname{Ker}\left(\gamma^{*}\right)$ est fini.

2. Dans le cas arithmétique, $\operatorname{Ker}\left(\gamma^{*}\right)$ est inclus dans $\widetilde{U_{T}}$.

3. Dans le cas fonctionnel, $\operatorname{Ker}\left(\gamma^{*}\right) \cap\left(T\left(\boldsymbol{A}_{K}\right) / T\left(\boldsymbol{A}_{K}\right)^{1}\right)^{*}$ est de cardinal $\mathcal{K}_{T}$.

Démonstration : D'après (3.13) et le lemme 4.2, on a une suite exacte

$$
T_{P_{\Sigma}}\left(\mathbf{A}_{K}\right) / T_{P_{\Sigma}}(K) \longrightarrow T\left(\mathbf{A}_{K}\right) / T(K) \longrightarrow A(T) \longrightarrow 0
$$

d'où par dualité une suite exacte

$$
0 \longrightarrow A(T)^{*} \longrightarrow\left(T\left(\mathbf{A}_{K}\right) / T(K)\right)^{*} \longrightarrow\left(T_{P_{\Sigma}}\left(\mathbf{A}_{K}\right) / T_{P_{\Sigma}}(K)\right)^{*}
$$

d'où le premier point.

Dans le cas arithmétique, le fait que $\operatorname{Ker}\left(\gamma^{*}\right)$ soit fini et que $\left(T\left(\mathbf{A}_{K}\right) / T\left(\mathbf{A}_{K}\right)^{1}\right)^{*}$ soit sans torsion donne aussitôt le second point.

Dans le cas fonctionnel, on note que le groupe $\operatorname{Ker}\left(\gamma^{*}\right) \cap\left(T\left(\mathbf{A}_{K}\right) / T\left(\mathbf{A}_{K}\right)^{1}\right)^{*}$ est le noyau du morphisme

$$
\left(T\left(\mathbf{A}_{K}\right) / T\left(\mathbf{A}_{K}\right)^{1}\right)^{*} \longrightarrow\left(T_{P_{\Sigma}}\left(\mathbf{A}_{K}\right) / T_{P_{\Sigma}}\left(\mathbf{A}_{K}\right)^{1}\right)^{*}
$$

induit par la composition avec $\gamma_{\mathbf{A}_{K}}$, et qu'on a un diagramme commutatif

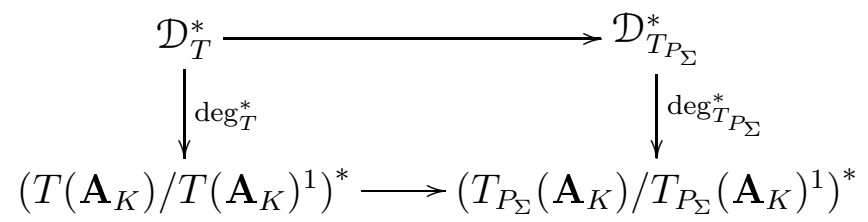

dont les flèches verticales sont des isomorphismes. Comme $\mathcal{K}_{T}$ est le cardinal du conoyau du dual du morphisme $\mathcal{D}_{T}^{*} \longrightarrow \mathcal{D}_{T_{P_{\Sigma}}}^{*}$, on a le troisième point.

\subsubsection{Cas arithmétique}

Rappelons que $d \chi$ est par définition la mesure duale de $\widetilde{\omega_{T}}$, où $\widetilde{\omega_{T}}$ est la mesure quotient de $\omega_{T}$ sur $T\left(\mathbf{A}_{K}\right) / T(K), T(K)$ étant muni de la mesure discrète.

Comme $\left(T\left(\mathbf{A}_{K}\right)^{1} / T(K)\right)^{*}$ est discret, $\left(T\left(\mathbf{A}_{K}\right) / T\left(\mathbf{A}_{K}\right)^{1}\right)^{*}$ est un sousgroupe ouvert de $\left(T\left(\mathbf{A}_{K}\right) / T(K)\right)^{*}$, et on a pour toute fonction $\varphi$ intégrable $\operatorname{sur}\left(T\left(\mathbf{A}_{K}\right) / T(K)\right)^{*}$

$$
\int_{\left(T\left(\mathbf{A}_{K}\right) / T(K)\right)^{*}} \varphi(\chi) d \chi=\int_{\chi \in\left(T\left(\mathbf{A}_{K}\right) / T\left(\mathbf{A}_{K}\right)^{1}\right)^{*}}\left(\sum_{\chi^{\prime} \in \widetilde{\mathcal{U}_{T}}} \varphi\left(\chi \cdot \chi^{\prime}\right)\right) d \chi
$$


En vue d'obtenir une formule intégrale explicite, il nous faut déterminer la restriction de la mesure de Haar $d \chi$ au sous-groupe ouvert $\left(T\left(\mathbf{A}_{K}\right) / T\left(\mathbf{A}_{K}\right)^{1}\right)^{*}$.

Rappelons qu'on a défini la mesure $\omega_{T}^{1}$ sur $T\left(\mathbf{A}_{K}\right)^{1} / T(K)$ par la relation

$$
\widetilde{\omega_{T}}=\omega_{T}^{1}\left(\operatorname{deg}_{T}^{-1}\right)_{*}(d t),
$$

où $d t$ est la mesure de Lebesgue sur $\left(X(T)^{G}\right)_{\mathbf{R}}^{\vee}$ normalisée par le réseau $\left(X(T)^{G}\right)^{\vee}$.

Si on note $\left(\omega_{T}^{1}\right)^{*}$ et $d t^{*}$ les mesures duales de $\omega_{T}^{1}$ et $d t$ respectivement, on a donc

$$
d \chi=\left(\omega_{T}^{1}\right)^{*} \cdot\left(\left[\operatorname{deg}_{T}^{*}\right]^{-1}\right)_{*} d t^{*} .
$$

Comme $T\left(\mathbf{A}_{K}\right)^{1} / T(K)$ est compact, d'après le lemme $4.16\left(\omega_{T}^{1}\right)^{*}$ est la mesure de Haar sur le groupe discret $\left(T\left(\mathbf{A}_{K}\right)^{1} / T(K)\right)^{*}$ pour laquelle chaque point a pour masse

$$
\frac{1}{\int_{T\left(\mathbf{A}_{K}\right)^{1} / T(K)} \omega_{T}^{1}}=\frac{1}{b(T)}
$$

Par ailleurs, on note désormais $d y$ la mesure de Lebesgue sur $X(T)_{\mathbf{R}}^{G}$ (identifié au dual topologique de $\left(X(T)^{G}\right)_{\mathbf{R}}^{\vee}$ de la manière décrite au lemme 4.16), normalisée par le réseau $X(T)^{G}$. D'après le lemme 4.16, on a donc

$$
d y=\frac{d t^{*}}{(2 \pi)^{\operatorname{rg}\left(X(T)^{G}\right)}} .
$$

On obtient finalement le lemme suivant.

\section{Lemme 5.42}

Soit $\varphi$ une fonction intégrable sur $\left(T\left(\boldsymbol{A}_{K}\right) / T(K)\right)^{*}$. On a alors

$$
\int_{\left(T\left(\boldsymbol{A}_{K}\right) / T(K)\right)^{*}} \varphi(\chi) d \chi=\frac{1}{(2 \pi)^{\operatorname{rg}\left(X(T)^{G}\right)} b(T)} \int_{y \in X(T)_{\boldsymbol{R}}^{G}}\left(\sum_{\chi \in \widetilde{\mathcal{U}_{T}}} \varphi\left(\chi_{y} \cdot \chi\right)\right) d y
$$

Pour appliquer le lemme 4.20, on a besoin d'abord de prouver le caractère $\mathbf{L}^{1}$ de $\mathcal{F} H(-\boldsymbol{s},$.$) sur \left(T\left(\mathbf{A}_{K}\right) / \mathbf{K}(T) \cdot T(K)\right)^{*}$ (rappelons que cette intégrabilité sera automatique dans le cas fonctionnel, grâce à la compacité de $\left.\left(T\left(\mathbf{A}_{K}\right) / \mathbf{K}(T) \cdot T(K)\right)^{*}\right)$. Pour ce faire, on utilise de manière cruciale la majoration pour les transformées de Fourier locales aux places archimédiennes obtenue au corollaire 5.14, ainsi que la majoration uniforme des fonctions $L$ donnée par le résultat suivant, conséquence du résultat principal de [Ra]. 


\section{Proposition 5.43}

Soit $E$ un corps de nombres et $\delta>0$. Il existe un réel $\varepsilon>0$ (vérifiant $0<\varepsilon<\frac{1}{3}$ ) et une constante $C>0$ telle qu'on ait pour tout $s$ vérifiant $\Re(s)>1-\varepsilon$ et tout caractère $\chi$ de $\boldsymbol{G}_{m}\left(\boldsymbol{A}_{E}\right)$ trivial sur $\boldsymbol{K}\left(\boldsymbol{G}_{m}\right)$ la majoration

$$
\left|\frac{s-1}{s} L_{E}(\chi, s)\right| \leqslant C(1+|\Im(s)|)^{\delta}\left(1+\| \chi_{\infty}||\right)^{\delta}
$$

La proposition suivante est la proposition B.3 de [CLTs].

\section{Proposition 5.44 (Chambert-Loir, Tschinkel)}

Soit $V$ un $\boldsymbol{R}$-espace -vectoriel de dimension finie muni d'une norme $\|$. $\|,\left(\ell_{j}\right)$ une base du dual de $V, M$ un sous-espace vectoriel de $V$, dm une mesure de Lebesgue sur $M, V^{\prime}$ un supplémentaire de $M$ dans $V$, et $\delta$ un réel vérifiant $0<\delta<1$. Pout tout $\delta^{\prime}>\delta$, il existe une constante $C>0$ et un ensemble fini $\left(\ell_{i, j}\right)_{i \in I, j \in J}$ d'éléments du dual de $V^{\prime}$ tels que :

- pour tout $i \in I$, la famille $\left(\ell_{i, j}\right)_{j \in J}$ restreinte à $V$ est une base $d u$ dual de $V^{\prime}$;

- pour tous $v_{1}$ et $v_{2}$ dans $V$ on a la majoration

$$
\begin{aligned}
\int_{M} \frac{1}{\left(1+|| v_{1}+m||\right)^{1-\delta}} & \frac{d m}{\prod\left(1+\left|\ell_{j}\left(v_{2}+m\right)\right|\right)} \\
& \leqslant \frac{C}{\left(1+|| v_{1}||\right)^{1-\delta^{\prime}}} \sum_{i \in I} \frac{1}{\prod_{j \in J}\left(1+\left|\ell_{i, j}\left(v_{2}\right)\right|\right)}
\end{aligned}
$$

Nous aurons également besoin des résultats suivants sur le morphisme «type à l’infini».

\section{Lemme 5.45}

1. La restriction du morphisme «type à l'infini» à $\left(T\left(\boldsymbol{A}_{K}\right) / \mathbf{K}(T) \cdot T(K)\right)^{*}$ est de noyau fini.

2. La composition de l'isomorphisme

$$
\operatorname{deg}_{T}^{*}: X(T)_{\boldsymbol{R}}^{G} \stackrel{\sim}{\longrightarrow}\left(T\left(\boldsymbol{A}_{K}\right) / T\left(\boldsymbol{A}_{K}\right)^{1}\right)^{*}
$$

avec le morphisme de $\left(T\left(\boldsymbol{A}_{K}\right) / T\left(\boldsymbol{A}_{K}\right)^{1}\right)^{*}$ vers $X(T)_{\boldsymbol{R}, \infty}$ induit par le morphisme «type à l'infini» coüncide avec l'injection diagonale $X(T)_{\boldsymbol{R}}^{G} \rightarrow$ $X(T)_{\mathbf{R}, \infty}$.

3. On note $\mathfrak{U}_{T, \infty}$ l'image dans $X(T)_{\boldsymbol{R}, \infty}$ de $\mathcal{U}_{T}$ par le morphisme «type à l'infini». Alors $\mathcal{U}_{T, \infty}$ est un réseau d'un sous-espace vectoriel supplémentaire de $X(T)_{\mathbf{R}}^{G}$ dans $X(T)_{\mathbf{R}, \infty}$. 
Démonstration : D'après la proposition 3.24, le groupe

$$
T\left(\mathbf{A}_{K}\right) / \mathbf{K}(T) T(K) T\left(\mathbf{A}_{K}\right)_{\mathcal{P}_{K, \infty}}
$$

est fini. Par dualité, ceci montre le point 1.

Le point 2 découle par dualité de la commutativité du diagramme

$$
\begin{gathered}
\prod_{v \in \mathcal{P}_{K, \infty}} T\left(K_{v}\right) / T\left(\mathcal{O}_{v}\right) \longrightarrow T\left(\mathbf{A}_{K}\right) / T\left(\mathbf{A}_{K}\right)^{1} \\
\downarrow_{\sum_{v} \operatorname{deg}_{T, v}} \longrightarrow \operatorname{deg}_{T} \\
\bigoplus_{v \in \mathcal{P}_{K, \infty}} \operatorname{Hom}\left(X(T)^{G_{v}}, \mathbf{R}\right) \longrightarrow \operatorname{Hom}\left(X(T)^{G}, \mathbf{R}\right)
\end{gathered}
$$

où la flèche horizontale du bas est la somme des restrictions.

Notons

$$
\varepsilon: \prod_{v \in \mathcal{P}_{K, \infty}} T\left(K_{v}\right) / T\left(\mathcal{O}_{v}\right) \longrightarrow \operatorname{Hom}\left(X(T)^{G}, \mathbf{R}\right)
$$

la composition de la flèche verticale de gauche et de la flèche horizontale du bas du diagramme (5.18).

Le groupe $T\left(\mathbf{A}_{K}\right)_{\mathcal{P}_{K, \infty}}^{1} / \mathbf{K}(T) \cdot T(K) \cap T\left(\mathbf{A}_{K}\right)_{\mathcal{P}_{K, \infty}}^{1}$ est un sous-groupe ouvert du groupe $T\left(\mathbf{A}_{K}\right)^{1} / \mathbf{K}(T) \cdot T(K)$, et donc un sous-groupe fermé. D'après la proposition 3.24, $T\left(\mathbf{A}_{K}\right)_{\mathcal{P}_{K, \infty}}^{1} / \mathbf{K}(T) \cdot T(K) \cap T\left(\mathbf{A}_{K}\right)_{\mathcal{P}_{K, \infty}}^{1}$ est donc compact.

Notons

$$
T\left(\mathcal{O}_{K}\right)=T(K) \cap \prod_{v \in \mathcal{P}_{K, f}} T\left(\mathcal{O}_{v}\right)
$$

et $T\left(\mathcal{O}_{K}\right)_{\infty}$ l'image de $T\left(\mathcal{O}_{K}\right)$ dans $\prod_{v \in \mathcal{P}_{K, \infty}} T\left(K_{v}\right) / T\left(\mathcal{O}_{v}\right)$. Ainsi $T\left(\mathcal{O}_{K}\right)_{\infty}$ est un sous-groupe discret de $\prod_{v \in \mathcal{P}_{K, \infty}} T\left(K_{v}\right) / T\left(\mathcal{O}_{v}\right)$ dont l'image est contenue dans $\operatorname{Ker}(\varepsilon)$.

On a

$$
\mathbf{K}(T) \cdot T(K) \cap T\left(\mathbf{A}_{K}\right)_{\mathcal{P}_{K, \infty}}^{1}=T\left(\mathcal{O}_{K}\right) . \prod_{v \in \mathcal{P}_{K, \infty}} T\left(\mathcal{O}_{v}\right)
$$

et un isomorphisme

$$
T\left(\mathbf{A}_{K}\right)_{\mathcal{P}_{K, \infty}}^{1} / \mathbf{K}(T) \cdot T(K) \cap T\left(\mathbf{A}_{K}\right)_{\mathcal{P}_{K, \infty}}^{1} \stackrel{\sim}{\longrightarrow} \operatorname{Ker}(\varepsilon) / T\left(\mathcal{O}_{K}\right)_{\infty}
$$

Ainsi $\operatorname{Ker}(\varepsilon) / T\left(\mathcal{O}_{K}\right)_{\infty}$ est compact et $T\left(\mathcal{O}_{K}\right)_{\infty}$ est un réseau de $\operatorname{Ker}(\varepsilon)$ (lorsque $K=\mathbf{G}_{m}$, ce résultat est le théorème des unités de Dirichlet). 
Le diagramme commutatif suivant

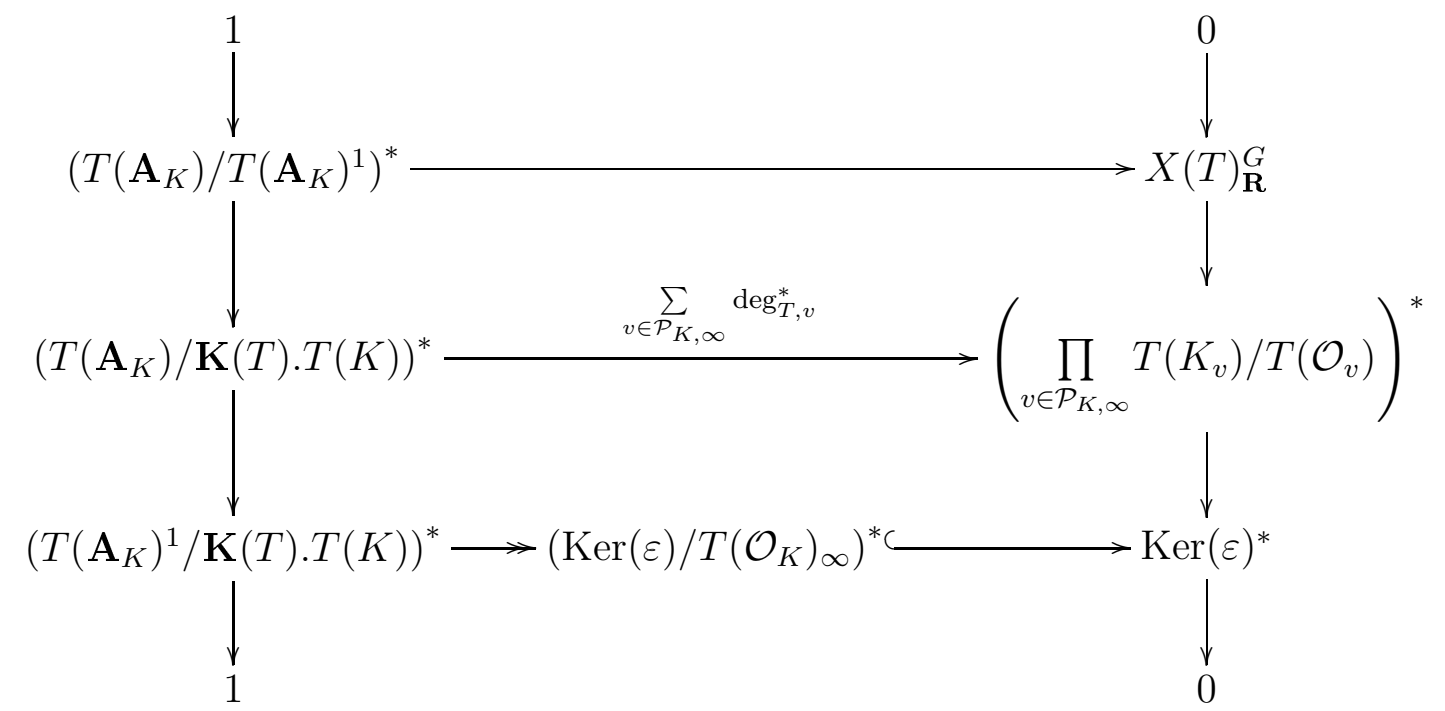

où les deux colonnes sont exactes, montre alors le point 3 .

Pour alléger les notations, on identifie dans l'énoncé et la démonstration de la proposition 5.46 l'espace vectoriel $X(T)_{\mathbf{R}}^{G}$ à son image par $\gamma_{\mathbf{R}}$ dans $\operatorname{PL}(\Sigma)_{\mathbf{R}}^{G}$.

\section{Proposition 5.46}

1. La série de fonctions holomorphes

$$
\boldsymbol{s} \mapsto \sum_{\chi \in \mathcal{U}_{T}}\left(\prod_{\alpha \in \Sigma(1) / G} L_{K_{\alpha}}\left(\chi_{\alpha}, s_{\alpha}\right)\right) f(\chi, s) f_{\infty}(\chi, s)
$$

converge uniformément sur tout compact de $\mathcal{T}\left(\boldsymbol{R}_{>1}^{\Sigma(1) / G}\right)$ et définit donc une fonction holomorphe sur ce domaine, notée $G(s)$.

2. On note $F$ la fonction décalée

$$
s \mapsto \frac{1}{[A(T)]} G\left(s-\varphi_{0}\right) .
$$

Il existe alors un réel $\varepsilon>0$ tel que la fonction

$$
\boldsymbol{s} \mapsto\left(\prod_{\alpha \in \Sigma(1)} s_{\alpha}\right) F(\boldsymbol{s})
$$


se prolonge en une fonction holomorphe $\operatorname{sur} \mathcal{T}\left(\boldsymbol{R}_{>-\varepsilon}^{\Sigma(1) / G}\right)$, dont la valeur en $s=0$ est

$$
\lim _{s \rightarrow 1}(s-1)^{[\Sigma(1) / G]} \int_{\overline{T(K)} \cap T\left(\boldsymbol{A}_{K}\right)} H\left(-s \varphi_{0}, t\right) \omega_{T}(t) .
$$

3. Soit $\delta>0$. Il existe un réel $\varepsilon>0$ tel que la propriété suivante est vérifiée : pour tout $\delta^{\prime}$ vérifiant $0<\delta^{\prime}<1$ et tout compact $\boldsymbol{K}$ de $\boldsymbol{R}_{>-\varepsilon}^{\Sigma(1) / G}$, il existe une constante $C>0$ et un ensemble fini $\left(\ell_{i j}\right)_{i \in I, j \in J}$ de formes linéaires sur $\boldsymbol{R}^{\Sigma(1) / G}$ tels que :

- pour tout $i \in I$, la famille $\left(\ell_{i j}\right)_{j \in J}$ restreinte à $X(T)_{\boldsymbol{R}}^{G}$ est une base du dual de $X(T)_{\mathbf{R}}^{G}$;

- pour tout $\boldsymbol{s}$ de $\mathcal{T}(\boldsymbol{K})$ et tout $y \in X(T)_{\boldsymbol{R}}^{G}$, on a la majoration

$$
\begin{aligned}
& \left|\left(\prod_{\alpha \in \Sigma(1) / G} \frac{s_{\alpha}+i y_{\alpha}}{1+s_{\alpha}+i y_{\alpha}}\right) F(s+i y)\right| \\
& \leqslant C \frac{(1+\|\Im(s)\|)^{1+\delta}}{(1+\|y\|)^{1-\delta^{\prime}}} \sum_{i \in I} \prod_{j \in J} \frac{1}{1+\left|\ell_{i, j}(\Im(s)+y)\right|} .
\end{aligned}
$$

Remarques 5.47:

1. La démonstration qui suit est fortement inspirée de [CLTs].

2. Pour $s \in \mathcal{T}\left(\mathbf{R}_{>0}^{\Sigma(1) / G}\right)$, soit

$$
\widetilde{G}(\boldsymbol{s})=\sum_{\chi \in \mathcal{U}_{T}}\left|\left(\prod_{\alpha \in \Sigma(1) / G} L_{K_{\alpha}}\left(\chi_{\alpha}, s_{\alpha}\right)\right) f(\chi, s) f_{\infty}(\chi, s)\right|
$$

et $\widetilde{F}(\boldsymbol{s})=\frac{1}{[A(T)]} \widetilde{G}\left(\boldsymbol{s}-\varphi_{0}\right)$. La démonstration de la proposition 5.46 montrera en fait que $\widetilde{F}$ vérifie la majoration (5.28).

3. Le point 3 montre que la fonction

$$
\boldsymbol{s} \mapsto\left(\prod_{\alpha \in \Sigma(1) / G} \frac{s_{\alpha}}{1+s_{\alpha}}\right) F(s)
$$

est $X(T)_{\mathbf{R}}^{G}$-contrôlée au sens de [CLTs, Définition 3.13].

Démonstration : 
D'après la proposition 2.1 et les lemmes $5.1,5.32$ et 5.33 , pour tout $\chi$, la fonction

$$
\boldsymbol{s} \mapsto\left(\prod_{\alpha \in \Sigma(1) / G} \frac{s_{\alpha}-1}{s_{\alpha}} L_{K_{\alpha}}\left(\chi_{\alpha}, s_{\alpha}\right)\right) f(\chi, s) f_{\infty}(\chi, s)
$$

se prolonge en une une fonction holomorphe sur $\mathcal{T}\left(\mathbf{R}_{>\frac{1}{2}}^{\Sigma(1) / G}\right)$, que l'on note $g(\chi, s)$.

Montrons alors qu'il existe un réel $\varepsilon$ vérifiant $0<\varepsilon<\frac{1}{2}$ tel que la série de fonctions

$$
\sum_{\chi \in \mathcal{U}_{T}} g(\chi, s)
$$

converge uniformément sur tout compact de $\mathcal{T}\left(\mathbf{R}_{>1-\varepsilon}^{\Sigma(1) / G}\right)$, ce qui donnera les points 1 et 2, à l'exception de (5.27).

Soit $\delta>0$. D'après la proposition 5.43, il existe un réel $\varepsilon>0$ et une constante $C_{1}>0$ telle qu'on ait, pour tout $\boldsymbol{s} \in \mathcal{T}\left(\mathbf{R}_{>1-\varepsilon}^{\Sigma(1) / G}\right)$ et tout $\chi \in$ $\left(T\left(\mathbf{A}_{K}\right) / \mathbf{K}(T)\right)^{*}$

$\left|\prod_{\alpha \in \Sigma(1) / G}\left(\frac{s_{\alpha}-1}{s_{\alpha}}\right) L_{K_{\alpha}}\left(s_{\alpha}, \chi_{\alpha}\right)\right| \leqslant C_{1} \prod_{\alpha \in \Sigma(1) / G}\left(1+\left|\Im\left(s_{\alpha}\right)\right|\right)^{\delta}\left(1+\left\|\left(\chi_{\alpha}\right)_{\infty}\right\|\right)^{\delta}$

soit

$$
\left|\prod_{\alpha \in \Sigma(1) / G}\left(\frac{s_{\alpha}-1}{s_{\alpha}}\right) L_{K_{\alpha}}\left(s_{\alpha}, \chi_{\alpha}\right)\right| \leqslant C_{1}(1+\|\Im(s)\|)^{\delta}\left(1+\left\|\chi_{\infty}\right\|\right)^{\delta} .
$$

Soit $\mathbf{K}$ un compact de $\mathcal{T}\left(\mathbf{R}_{>1-\varepsilon}^{\Sigma(1) / G}\right)$. D'après le corollaire 5.14, il existe une constante $C_{2}>0$ telle que pour tout $s \in \mathbf{K}$ et tout $\chi \in \mathcal{U}_{T}$ on a

$$
\left|f_{\infty}(\chi, s)\right| \leqslant \frac{C_{2}}{1+\left\|\chi_{\infty}\right\|} \sum_{\widetilde{\sigma} \in \Sigma_{\max }^{\infty}} \frac{1}{\prod_{i \in \widetilde{\sigma}(1)}\left(1+\left|\left\langle\rho_{i}, \chi_{\infty}\right\rangle+\Im\left(s_{i}\right)\right|\right)}
$$

D'après le lemme 5.35, il existe une constante $C_{3}$ telle que pour tout $s \in \mathbf{K}$ et tout $\chi \in \mathcal{U}_{T}$ on a

$$
|f(\chi, s)| \leqslant C_{3}
$$


Finalement, on a montré qu'il existe une constante $C>0$ telle que pour tout $s \in \mathbf{K}$ et tout $\chi \in \mathcal{U}_{T}$ on a la majoration

$$
|g(\chi, s)| \leqslant C \frac{1}{\left(1+|| \chi_{\infty}||\right)^{1-\delta}} \sum_{\widetilde{\sigma} \in \Sigma_{\max }^{\infty}} \frac{1}{\prod_{i \in \widetilde{\sigma}(1)}\left(1+\left|\left\langle\rho_{i}, \chi_{\infty}\right\rangle+\Im\left(s_{i}\right)\right|\right)}
$$

Pour montrer que la série de fonctions (5.32) converge uniformément sur $\mathbf{K}$ il suffit donc de montrer que pour tout $\widetilde{\sigma} \in \Sigma_{\max }^{\infty}$ la série

$$
\sum_{\chi \in \mathcal{U}_{T}} \frac{1}{\left(1+\| \chi_{\infty}||\right)^{1-\delta}} \frac{1}{\prod_{i \in \widetilde{\sigma}(1)}\left(1+\left|\left\langle\rho_{i}, \chi_{\infty}\right\rangle+\Im\left(s_{i}\right)\right|\right)}
$$

est convergente uniformément localement en $\boldsymbol{s}$.

D'après le lemme 5.45, il suffit de montrer que la série

$$
\sum_{m \in \mathcal{U}_{T, \infty}} \frac{1}{(1+\| m||)^{1-\delta}} \frac{1}{\prod_{i \in \widetilde{\sigma}(1)}\left(1+\left|\left\langle\rho_{i}, m\right\rangle+\Im\left(s_{i}\right)\right|\right)}
$$

est convergente uniformément localement en $\boldsymbol{s}$.

Mais cette série est majorée par l'intégrale

$$
\int_{m \in \mathcal{U}_{T, \infty} \otimes \mathbf{R}} \frac{1}{(1+|| m||)^{1-\delta}} \frac{d m}{\prod_{i \in \widetilde{\sigma}(1)}\left(1+\left|\left\langle\rho_{i}, m\right\rangle+\Im\left(s_{i}\right)\right|\right)},
$$

où $d m$ est la mesure de Lebesgue sur $\mathcal{U}_{T, \infty} \otimes \mathbf{R}$ normalisée par le réseau $\mathcal{U}_{T, \infty}$. Pour conclure, on applique alors la proposition 5.44 avec $V=X(T)_{\mathbf{R}, \infty}$, $M=\mathcal{U}_{T, \infty} \otimes \mathbf{R}, V^{\prime}=X(T)_{\mathbf{R}}^{G}$ (qui est bien un supplémentaire de $M$ dans $V$ d'après le point 3 du lemme 5.45) $v_{1}=0, v_{2}=\left(\Im\left(s_{i}\right)\right)_{i \in \widetilde{\sigma}(1)}$, et en prenant pour base du dual de $V$ la famille $\left(\left\langle\rho_{i}, .\right\rangle\right)_{i \in \widetilde{\sigma}(1)}$.

Montrons à présent (5.27). Il s'agit de montrer qu'on a

$$
\sum_{\chi \in \mathcal{U}_{T}} g\left(\chi, \varphi_{0}\right)=[A(T)] \lim _{s \rightarrow 1}(s-1)^{[\Sigma(1) / G]} \int_{\overline{T(K)} \cap T\left(\mathbf{A}_{K}\right)} H\left(-s \varphi_{0}, t\right) \omega_{T}(t) .
$$

Compte tenu de la définition de $g(\chi, s)$ et du fait que $L_{K_{\alpha}}\left(\chi_{\alpha},.\right)$ est holomorphe sur $\mathbf{C}$ si $\chi_{\alpha}$ est non trivial, $g\left(\chi, \varphi_{0}\right)$ est nul dès qu'il existe un $\alpha$ tel que $\chi_{\alpha}$ est non trivial, en d'autre termes dès que $\chi$ n'est pas dans $\operatorname{Ker}\left(\gamma^{*}\right)$. Le premier membre de (5.41) s'écrit donc

$$
\sum_{\chi \in \mathcal{U}_{T} \cap \operatorname{Ker}\left(\gamma^{*}\right)}\left(\prod_{\alpha \in \Sigma(1) / G} \operatorname{Res}_{s=1} \zeta_{K_{\alpha}}(s)\right) f\left(\chi, \varphi_{0}\right) f_{\infty}\left(\chi, \varphi_{0}\right)
$$


Par ailleurs, pour $s$ complexe tel que $\Re(s)>1$, appliquons la formule de Poisson 4.17 avec $\mathcal{G}=T\left(\mathbf{A}_{K}\right), \mathcal{H}=\overline{T(K)} \cap T\left(\mathbf{A}_{K}\right)$, dh $=d g=\omega_{T}$ et $F=$ $H\left(-s \varphi_{0},.\right)$. On obtient d'après le lemme 5.41 et le fait que $\mathcal{F} H\left(\chi,-s \varphi_{0}\right)$ est nulle dès que $\chi$ n'est pas trivial sur $\mathbf{K}(T)$

$$
\begin{aligned}
\int_{\overline{T(K)} \cap T\left(\mathbf{A}_{K}\right)} H\left(-s \varphi_{0}, t\right) \omega_{T}(t) & =\frac{1}{\left[\operatorname{Ker}\left(\gamma^{*}\right)\right]} \sum_{\chi \in \operatorname{Ker}\left(\gamma^{*}\right)} \mathcal{F} H\left(\chi,-s \varphi_{0}\right) \\
& =\frac{1}{[A(T)]} \sum_{\chi \in \operatorname{Ker}\left(\gamma^{*}\right) \cap \mathcal{U}_{T}} \mathcal{F} H\left(\chi,-s \varphi_{0}\right)
\end{aligned}
$$

Mais d'après (5.7) on a pour tout $\chi \in \operatorname{Ker}\left(\gamma^{*}\right) \cap \mathcal{U}_{T}$

$$
\mathcal{F} H\left(\chi,-s \varphi_{0}\right)=\left(\prod_{\alpha \in \Sigma(1) / G} \zeta_{K_{\alpha}}(s)\right) f\left(\chi, s \varphi_{0}\right) f_{\infty}\left(\chi, s \varphi_{0}\right) .
$$

De (5.42), (5.44) et (5.45), on déduit aussitôt la relation (5.41).

Montrons à présent le point 3. Soit $\delta>0$. D'après la proposition 5.43, il existe un réel $\varepsilon>0$ et une constante $C_{1}>0$ telle qu'on ait, pour tout $\boldsymbol{s} \in \mathcal{T}\left(\mathbf{R}_{>-\varepsilon}^{\Sigma(1) / G}\right)$ et tout $\chi \in \mathcal{U}_{T}$

$$
\begin{aligned}
\left|\left(\prod_{\alpha \in \Sigma(1) / G} \frac{s_{\alpha}+i y_{\alpha}}{s_{\alpha}+1+i y_{\alpha}}\right) L_{K_{\alpha}}\left(\chi_{\alpha}, s_{\alpha}+1+i y_{\alpha}\right)\right| \\
\leqslant C_{1}(1+\|\Im(s)+y\|)^{\delta}\left(1+\left\|\chi_{\infty}\right\|\right)^{\delta} .
\end{aligned}
$$

Soit $\mathbf{K}$ un compact de $\mathbf{R}_{>-\varepsilon}^{\Sigma(1) / G}$. D'après (5.46), le lemme 5.35 et le corollaire 5.14 , il existe une constante $C_{2}>0$ telle que pour tout $s \in \mathcal{T}(\mathbf{K})$, tout $y \in X(T)_{\mathbf{R}}^{G}$ et tout $\chi \in \mathcal{U}_{T}$ l'expression

$$
\left|g\left(\chi,\left(s_{\alpha}-1+i y_{\alpha}\right)\right)\right|=\left|g\left(\chi \cdot \chi_{y}, s-\varphi_{0}\right)\right|
$$

est majorée par

$$
C_{2} \frac{(1+\|\Im(s)+y\|)^{\delta}\left(1+\left\|\chi_{\infty}\right\|\right)^{\delta}}{1+\left\|\chi_{\infty}+y\right\|} \sum_{\widetilde{\sigma} \in \Sigma_{\max }^{\infty}} \frac{1+\sum_{i \in \widetilde{\sigma}(1)}\left|\Im\left(s_{i}\right)\right|}{\prod_{i \in \widetilde{\sigma}(1)}\left(1+\left|\left\langle\rho_{i}, \chi_{\infty}+y\right\rangle+\Im\left(s_{i}\right)\right|\right)} .
$$


On en déduit que pour tout $\boldsymbol{s} \in \mathcal{T}(\mathbf{K})$ et tout $y \in X(T)_{\mathbf{R}}^{G}$, l'expression

$$
\left|\left(\prod_{\alpha \in \Sigma(1) / G} \frac{s_{\alpha}+i y_{\alpha}}{s_{\alpha}+1+i y_{\alpha}}\right) F(s+i y)\right|
$$

est majorée par

$$
C_{2}(1+\|\Im(s)+y\|)^{\delta} \sum_{\widetilde{\sigma} \in \Sigma_{\max }^{\infty}}\left(1+\sum_{i \in \widetilde{\sigma}(1)}\left|\Im\left(s_{i}\right)\right|\right) \phi_{\widetilde{\sigma}}(s, y)
$$

où $\phi_{\widetilde{\sigma}}(s, y)$ est défini par la série

$$
\phi_{\widetilde{\sigma}}(\boldsymbol{s}, y)=\sum_{\chi \in \mathcal{U}_{T}} \frac{\left(1+\left\|\chi_{\infty}\right\|\right)^{\delta}}{1+\left\|\chi_{\infty}+y\right\|} \prod_{i \in \widetilde{\sigma}(1)} \frac{1}{1+\left|\left\langle\rho_{i}, \chi_{\infty}\right\rangle+\Im\left(s_{i}\right)+y_{i}\right|} .
$$

On obtient finalement que l'expression (5.49) est majorée par

$$
C_{2}(1+\|\Im(s)\|)^{1+\delta}(1+\|y\|)^{1+\delta} \sum_{\widetilde{\sigma} \in \Sigma_{\max }^{\infty}} \phi_{\widetilde{\sigma}}(s, y) .
$$

Fixons $\widetilde{\sigma} \in \Sigma_{\max }^{\infty}$. De la majoration

$$
1+\left\|\chi_{\infty}\right\| \leqslant 1+\left\|\chi_{\infty}+y\right\|+\|y\| \leqslant\left(1+\left\|\chi_{\infty}+y\right\|\right)(1+\|y\|),
$$

on déduit qu'on a

$$
\phi_{\widetilde{\sigma}}(s, y) \leqslant \sum_{\chi \in \mathcal{U}_{T}} \frac{(1+\|y\|)^{\delta}}{\left(1+\left\|\chi_{\infty}+y\right\|\right)^{1-\delta}} \prod_{i \in \widetilde{\sigma}(1)} \frac{1}{1+\left|\left\langle\rho_{i}, \chi_{\infty}\right\rangle+\Im\left(s_{i}\right)+y_{i}\right|} .
$$

D'après le lemme 5.45, il existe alors une constante $C_{3}>0$ telle qu'on ait

$$
\phi_{\widetilde{\sigma}}(s, y) \leqslant C_{3} \sum_{m \in \mathcal{U}_{T, \infty}} \frac{(1+\|y\|)^{\delta}}{(1+\| m+y||)^{1-\delta}} \prod_{i \in \widetilde{\sigma}(1)} \frac{1}{1+\left|\left\langle\rho_{i}, m\right\rangle+\Im\left(s_{i}\right)+y_{i}\right|}
$$

soit

$$
\begin{aligned}
& \phi_{\widetilde{\sigma}}(\boldsymbol{s}, y) \\
& \leqslant C_{3}(1+\|y\|)^{\delta} \int_{m \in \mathcal{U}_{T, \infty} \otimes \mathbf{R}} \frac{1}{(1+|| m+y \|)^{1-\delta}} \frac{d m}{\prod_{i \in \widetilde{\sigma}(1)}\left(1+\left|\left\langle\rho_{i}, m\right\rangle+\Im\left(s_{i}\right)+y_{i}\right|\right)} .
\end{aligned}
$$

On conclut comme ci-dessus en appliquant la proposition 5.44 avec $V=$ $X(T)_{\mathbf{R}, \infty}, M=\mathcal{U}_{T, \infty}, V^{\prime}=X(T)_{\mathbf{R}}^{G}, v_{1}=y, v_{2}=\left(\Im\left(s_{i}\right)+y_{i}\right)_{i \in \widetilde{\sigma}(1)}$, et en prenant pour base du dual de $V$ la famille $\left(\left\langle\rho_{i}, .\right\rangle\right)_{i \in \widetilde{\sigma}(1)}$. 


\section{Corollaire 5.48}

Dans le cas arithmétique, pour tout $\boldsymbol{s} \in \mathcal{T}\left(\boldsymbol{R}_{>0}^{\Sigma(1) / G}\right)$ on a la représentation intégrale

$$
\sum_{t \in T(K)} H\left(t,-s-\varphi_{0}\right)=\frac{[A(T)]}{(2 \pi)^{\operatorname{rg}\left(X(T)^{G}\right)} b(T)} \int_{X(T)_{\boldsymbol{R}}^{G}} F\left(s-i \gamma_{\boldsymbol{R}}(y)\right) d y
$$

où $F$ est la fonction définie dans l'énoncé de la proposition 5.46.

Démonstration: On veut appliquer le lemme 4.20. On sait déjà (lemme 5.31) que $H\left(.,-\boldsymbol{s}-\varphi_{0}\right)$ est intégrable sur $T\left(\mathbf{A}_{K}\right)$, et il s'agit de montrer que $\chi \mapsto \mathcal{F} H\left(\chi,-\boldsymbol{s}-\varphi_{0}\right)$ est intégrable sur $\left(T\left(\mathbf{A}_{K}\right) / \mathbf{K}(T)\right)^{*}$. Or on a, d'après le lemme 5.42,

$$
\begin{aligned}
& \int_{\left(T\left(\mathbf{A}_{K}\right) / \mathbf{K}(T)\right)^{*}}\left|\mathcal{F} H\left(\chi,-s-\varphi_{0}\right)\right| d \chi \\
= & \frac{1}{(2 \pi)^{\operatorname{rg}\left(X(T)^{G}\right)} b(T)} \int_{X(T)_{\mathbf{R}}^{G}}\left(\sum_{\chi \in \mathcal{U}_{T}}\left|\mathcal{F} H\left(\chi_{y} \cdot \chi,-\boldsymbol{s}-\varphi_{0}\right)\right|\right) d y \\
= & \frac{1}{(2 \pi)^{\operatorname{rg}\left(X(T)^{G}\right) b(T)}} \int_{X(T)_{\mathbf{R}}^{G}}\left(\sum_{\chi \in \mathcal{U}_{T}}\left|\mathcal{F} H\left(\chi,-\boldsymbol{s}+i \gamma_{\mathbf{R}}(y)-\varphi_{0}\right)\right|\right) d y \\
= & \frac{[A(T)]}{(2 \pi)^{\operatorname{rg}\left(X(T)^{G}\right) b(T)}} \int_{X(T)_{\mathbf{R}}^{G}} \widetilde{F}\left(s-i \gamma_{\mathbf{R}}(y)\right) d y
\end{aligned}
$$

où $\widetilde{F}$ est la fonction définie à la remarque 5.47. Cette même remarque montre en outre que la dernière intégrale est finie.

On peut donc appliquer le lemme 4.20, ce qui donne la formule 5.57, compte tenu du lemme 5.42.

La technique pour évaluer le comportement analytique de la fonction définie par l'intégrale apparaissant dans (5.57) est développée par Batyrev et Tschinkel dans [BaTs2], et raffinée par Chambert-Loir et Tschinkel dans [CLTs]. Dans la section 6, nous rappelons le lemme technique principal de [CLTs]. Puis nous expliquons dans la section 8.2 comment appliquer ce résultat à l'évaluation de cette intégrale et donc à la fonction zêta des hauteurs des variétés toriques (ceci est également expliqué dans [CLTs], à ceci près que seules des variétés toriques déployées sont considérées; l'adaptation au cas non déployé est cependant aisée). 


\subsubsection{Cas fonctionnel}

Rappelons que $d \chi$ est par définition la mesure duale de $\widetilde{\omega_{T}}$, où $\widetilde{\omega_{T}}$ est la mesure quotient de $\omega_{T}$ sur $T\left(\mathbf{A}_{K}\right) / T(K), T(K)$ étant muni de la mesure discrète. Comme dans le cas arithmétique, en vue d'obtenir une formule intégrale explicite, il nous faut déterminer la restriction de la mesure de Haar $d \chi$ au sous-groupe ouvert $\left(T\left(\mathbf{A}_{K}\right) / T\left(\mathbf{A}_{K}\right)^{1}\right)^{*}$.

Rappelons que $\left(T\left(\mathbf{A}_{K}\right) / T\left(\mathbf{A}_{K}\right)^{1}\right)^{*}$ est un sous-groupe de $\left(T\left(\mathbf{A}_{K}\right) / \mathbf{K}(T) \cdot T(K)\right)^{*}$ d'indice fini égal au cardinal de $T\left(\mathbf{A}_{K}\right)^{1} / \mathbf{K}(T) . T(K)$. Si on note $\operatorname{cl}(T)$ ce cardinal, on a donc

$$
\int_{\left(T\left(\mathbf{A}_{K}\right) / T\left(\mathbf{A}_{K}\right)^{1}\right)^{*}} d \chi=\frac{1}{\mathrm{cl}(T)} \int_{\left(T\left(\mathbf{A}_{K}\right) / \mathbf{K}(T) \cdot T(K)\right)^{*}} d \chi .
$$

Appliquons à présent le corollaire 4.18 de la formule de Poisson avec $\mathcal{G}=$ $T\left(\mathbf{A}_{K}\right), d g=\omega_{T}, \mathcal{H}=T(K)$, dh la mesure discrète, $\mathcal{K}=\mathbf{K}(T)$ (rappelons que $\int \omega_{T}$ est non nul, cf. section 3.9) et $F$ l'indicatrice de $\mathbf{K}(T)$, on obtient $\mathbf{K}(T)$

$$
[\mathbf{K}(T) \cap T(K)]=\int_{\mathbf{K}(T)} \omega_{T} \int_{\left(T\left(\mathbf{A}_{K}\right) / \mathbf{K}(T) \cdot T(K)\right)^{*}} d \chi .
$$

Or on a

$$
\begin{aligned}
\int_{\mathbf{K}(T)} \omega_{T} & =[\mathbf{K}(T) \cap T(K)] \int_{\mathbf{K}(T) \cdot T(K) / T(K)} \omega_{T}^{1} \\
& =\frac{[\mathbf{K}(T) \cap T(K)]}{\operatorname{cl}(T)} \int_{T\left(\mathbf{A}_{K}\right)^{1} / T(K)} \omega_{T}^{1} \\
& \left.=\frac{[\mathbf{K}(T) \cap T(K)]}{\operatorname{cl}(T)} \log (q)^{\operatorname{rg}\left(X(T)^{G}\right.}\right) b(T) .
\end{aligned}
$$

En combinant (5.62), (5.63), et (5.66), on obtient

$$
\begin{aligned}
\int_{\left(T\left(\mathbf{A}_{K}\right) / T\left(\mathbf{A}_{K}\right)^{1}\right)^{*}} d \chi & =\frac{1}{\operatorname{cl}(T)}[\mathbf{K}(T) \cap T(K)] \frac{\operatorname{cl}(T)}{[\mathbf{K}(T) \cap T(K)] \log (q)^{\operatorname{rg}\left(X(T)^{G}\right)} b(T)} \\
& =\frac{1}{\log (q)^{\operatorname{rg}\left(X(T)^{G}\right) b(T)}} .
\end{aligned}
$$

Soit $\widetilde{d \chi}$ la mesure de Haar sur $\left(T\left(\mathbf{A}_{K}\right) / T\left(\mathbf{A}_{K}\right)^{1}\right)^{*}$ de masse totale 1. On déduit de ce qui précède le lemme suivant. 
Lemme 5.49

Pour toute fonction $\varphi$ intégrable sur $\left(T\left(\boldsymbol{A}_{K}\right) / T(K)\right)^{*}$, on a

$$
\int_{\left(T\left(\boldsymbol{A}_{K}\right) / T(K)\right)^{*}} \varphi(\chi) d \chi=\frac{1}{\log (q)^{\operatorname{rg}\left(X(T)^{G}\right)} b(T)} \sum_{\chi^{\prime} \in \widetilde{\mathcal{U}_{T}}} \int_{\left(T\left(\boldsymbol{A}_{K}\right) / T\left(\boldsymbol{A}_{K}\right)^{1}\right)^{*}} \varphi\left(\chi \cdot \chi^{\prime}\right) \widetilde{d \chi}
$$

Convention 5.50: Soit $N$ un $\mathbf{Z}$-module libre de rang fini. On munira toujours $N_{\boldsymbol{U}}$ de la mesure de Haar $d \boldsymbol{z}$ de volume total 1. En d'autres termes, dans toute expression du type

$$
\int_{N_{U}} \ldots d \boldsymbol{z}
$$

il sera toujours sous-entendu que $d \boldsymbol{z}$ est la mesure de Haar de volume total 1.

Pour tout élément $n^{\vee}$ de $N^{\vee}$, on a donc

$$
\int_{N_{U}}\left\langle\boldsymbol{z}, n^{\vee}\right\rangle d \boldsymbol{z}= \begin{cases}0 & \text { si } n^{\vee} \neq 0 \\ 1 & \text { si } n^{\vee}=0\end{cases}
$$

\section{Lemme 5.51}

Soient $M$ un $Z$-module libre de rang fini, $N$ un sous-groupe de $M$ d'indice fini, et $\varphi$ une fonction intégrable sur $M_{\boldsymbol{U}}$. On a alors

$$
\int_{N_{U}} \varphi(\boldsymbol{z}) d \boldsymbol{z}=\int_{M_{U}} \varphi(\boldsymbol{z}) d \boldsymbol{z} .
$$

Démonstration: Soit $\left(e_{i}\right)_{i \in I}$ une base de $M$ adaptée à $N$. Au moyen de cette base, on identifie $M_{\boldsymbol{U}}$ à $\boldsymbol{U}^{I}$. Si $d z$ désigne la mesure uniforme sur $\boldsymbol{U}$, le membre de droite de (5.72) s'écrit alors

$$
\int_{\boldsymbol{U}^{I}} \varphi\left(z_{i}\right)_{i \in I} \underset{i \in I}{\otimes} d z_{i}
$$

Soit $\left(d_{i}\right) \in \mathbf{N}_{>0}^{I}$ tel que $\left(d_{i} e_{i}\right)$ soit une base de $N$. Au moyen de cette base, on identifie $N_{\boldsymbol{U}}$ à $\boldsymbol{U}^{I}$. Le membre de gauhe de (5.72) s'écrit alors

$$
\int_{\boldsymbol{U}^{I}} \varphi\left(z_{i}^{d_{i}}\right)_{i \in I} \underset{i \in I}{\otimes} d z_{i}
$$


Pour conclure, on remarque que si $d$ est un entier non nul on a pour toute fonction $\psi$ intégrable sur $\boldsymbol{U}$

$$
\int_{\boldsymbol{U}} \psi\left(z^{d}\right) d z=\int_{\boldsymbol{U}} \psi(z) d z .
$$

\section{Corollaire 5.52}

Pour toute fonction $\varphi$ intégrable sur $\left(T\left(\boldsymbol{A}_{K}\right) / T\left(\boldsymbol{A}_{K}\right)^{1}\right)^{*}$, on a

$$
\int_{\left(T\left(\boldsymbol{A}_{K}\right) / T\left(\boldsymbol{A}_{K}\right)^{1}\right)^{*}} \varphi(\chi) \widetilde{d \chi}=\int_{X(T)_{U}^{G}} \varphi\left(\chi_{\boldsymbol{z}}\right) d \boldsymbol{z}
$$

Démonstration : On applique le lemme 5.51 avec $M=\left(T\left(\mathbf{A}_{K}\right) / T\left(\mathbf{A}_{K}\right)^{1}\right)^{\vee}$ et $N=X(T)^{G}$.

\section{Lemme 5.53}

Pour $\boldsymbol{s} \in \mathcal{T}\left(\boldsymbol{R}_{>1}^{\Sigma(1) / G}\right)$, on a l'égalité

$$
\begin{gathered}
\sum_{\substack{\chi^{\prime} \in \mathcal{U}_{T} \\
\chi^{\prime} \in \operatorname{Ker}\left(\gamma^{*}\right) \cdot\left(T\left(\boldsymbol{A}_{K}\right) / T\left(\boldsymbol{A}_{K}\right)^{1}\right)^{*}}} \int_{\left(T\left(\boldsymbol{A}_{K}\right) / T\left(\boldsymbol{A}_{K}\right)^{1}\right)^{*}} \mathcal{F} H\left(\chi \cdot \chi^{\prime},-\boldsymbol{s}\right) \widetilde{d \chi} \\
=\frac{[A(T)]}{\left[\mathcal{K}_{T}\right]} \int_{\left(T\left(\boldsymbol{A}_{K}\right) / T\left(\boldsymbol{A}_{K}\right)^{1}\right)^{*}}\left[\int_{\left.\frac{T(K)}{T(T)} \boldsymbol{A}_{K}\right)} H(-\boldsymbol{s}, t) \chi(t) \omega_{T}(t)\right] \widetilde{d \chi}
\end{gathered}
$$

Démonstration : Soit $\chi \in\left(T\left(\mathbf{A}_{K}\right) / T\left(\mathbf{A}_{K}\right)^{1}\right)^{*}$ et $\boldsymbol{s} \in \mathcal{T}\left(\mathbf{R}_{>1}^{\Sigma(1) / G}\right)$. Appliquons le corollaire 4.18 de la formule de Poisson avec $\mathcal{G}=T\left(\mathbf{A}_{K}\right)$, $\mathcal{H}=\overline{T(K)} \cap T\left(\mathbf{A}_{K}\right), d h=d g=\omega_{T}, \mathcal{K}=\mathbf{K}(T)$ et $F=H(-\boldsymbol{s},.) \chi$.

On obtient, compte tenu du point $1 \mathrm{du}$ lemme 5.41, et du fait que la transformée de Fourier en un caractère non trivial sur $\mathbf{K}(T)$ est nulle

$$
\int_{\overline{T(K)} \cap T\left(\mathbf{A}_{K}\right)} H(-\boldsymbol{s}, t) \chi(t) \omega_{T}(t)=\frac{1}{[A(T)]} \sum_{\substack{\chi^{\prime} \in \operatorname{Ker}\left(\gamma^{*}\right) \\ \chi_{\mid \mathbf{K}(T)}^{\prime}=1}} \mathcal{F} H\left(\chi^{\prime} \cdot \chi,-s\right)
$$


On intègre à présent les deux membre de (5.78) $\operatorname{sur}\left(T\left(\mathbf{A}_{K}\right) / T\left(\mathbf{A}_{K}\right)^{1}\right)^{*}$ :

$$
\begin{aligned}
\int_{\left(T\left(\mathbf{A}_{K}\right) / T\left(\mathbf{A}_{K}\right)^{1}\right)^{*}} & {\left[\int_{\overline{T(K)} \cap T\left(\mathbf{A}_{K}\right)} H(-\boldsymbol{s}, t) \chi(t) \omega_{T}(t)\right] \widetilde{d \chi} } \\
& =\frac{1}{[A(T)]} \sum_{\substack{\chi^{\prime} \in \operatorname{Ker}\left(\gamma^{*}\right) \\
\chi_{\mid \mathbf{K}(T)}^{\prime}=1}} \int_{\left(T\left(\mathbf{A}_{K}\right) / T\left(\mathbf{A}_{K}\right)^{1}\right)^{*}} \mathcal{F} H\left(\chi^{\prime} \cdot \chi,-\boldsymbol{s}\right) \widetilde{d \chi} .
\end{aligned}
$$

Or on a d'après (5.3)

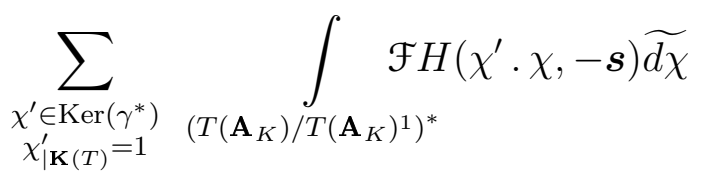

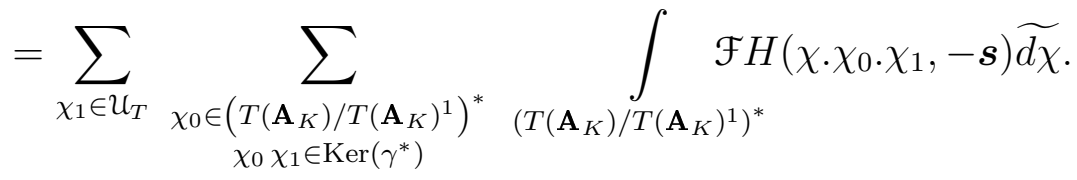

Comme $\widetilde{d \chi}$ est une mesure de Haar sur $\left(T\left(\mathbf{A}_{K}\right) / T\left(\mathbf{A}_{K}\right)^{1}\right)^{*}$, cette dernière expression se réécrit

$$
\sum_{\chi_{1} \in \mathcal{U}_{T}}\left[\left\{\chi_{0} \in\left(T\left(\mathbf{A}_{K}\right) / T\left(\mathbf{A}_{K}\right)^{1}\right)^{*}, \chi_{0} \chi_{1} \in \operatorname{Ker}\left(\gamma^{*}\right)\right\}\right] \int_{\left(T\left(\mathbf{A}_{K}\right) / T\left(\mathbf{A}_{K}\right)^{1}\right)^{*}} \mathcal{F} H\left(\chi \cdot \chi_{1},-\boldsymbol{s}\right) \widetilde{d \chi} .
$$

Pour conclure, on remarque que pour $\chi_{1} \in \mathcal{U}_{T}$, on a, si $\chi_{1} \notin \operatorname{Ker}\left(\gamma^{*}\right) .\left(T\left(\mathbf{A}_{K}\right) / T\left(\mathbf{A}_{K}\right)^{1}\right)^{*}$,

$$
\left[\left\{\chi_{0} \in\left(T\left(\mathbf{A}_{K}\right) / T\left(\mathbf{A}_{K}\right)^{1}\right)^{*}, \chi_{0} \chi_{1} \in \operatorname{Ker}\left(\gamma^{*}\right)\right\}\right]=0
$$

et, si $\chi_{1} \in \operatorname{Ker}\left(\gamma^{*}\right) .\left(T\left(\mathbf{A}_{K}\right) / T\left(\mathbf{A}_{K}\right)^{1}\right)^{*}$,

$$
\left[\left\{\chi_{0} \in\left(T\left(\mathbf{A}_{K}\right) / T\left(\mathbf{A}_{K}\right)^{1}\right)^{*}, \chi_{0} \chi_{1} \in \operatorname{Ker}\left(\gamma^{*}\right)\right\}\right]=\left[\left(T\left(\mathbf{A}_{K}\right) / T\left(\mathbf{A}_{K}\right)^{1}\right)^{*} \cap \operatorname{Ker}\left(\gamma^{*}\right)\right]
$$

soit d'après le point $3 \mathrm{du}$ lemme 5.41

$$
\left[\chi_{0} \in\left(T\left(\mathbf{A}_{K}\right) / T\left(\mathbf{A}_{K}\right)^{1}\right)^{*}, \chi_{0} \chi_{1} \in \operatorname{Ker}\left(\gamma^{*}\right)\right]=\mathcal{K}_{T} .
$$

Soit $I_{1}$ la fonction définie pour $\boldsymbol{s} \in \mathcal{T}\left(\mathbf{R}_{>1}^{\Sigma(1) / G}\right)$ par l'intégrale

$$
I_{1}(\boldsymbol{s})=\int_{X(T)_{U}^{G}}\left[\int_{\frac{T(K)}{T\left(T\left(\mathbf{A}_{K}\right)\right.}} H(-\boldsymbol{s}, t) \chi_{\boldsymbol{z}}(t) \omega_{T}(t)\right] d \boldsymbol{z} .
$$


Pour $\chi \in \mathcal{U}_{T}$, soit $I_{\chi}$ la fonction définie pour $s \in \mathcal{T}\left(\mathbf{R}_{>1}^{\Sigma(1) / G}\right)$ par l'intégrale

$$
I_{\chi}(\boldsymbol{s})=\int_{X(T)_{U}^{G}} \mathcal{F} H\left(\chi_{\boldsymbol{z}} \chi,-\boldsymbol{s}\right) d \boldsymbol{z}
$$

\section{Corollaire 5.54}

Dans le cas fonctionnel, pour tout élément $\boldsymbol{s} \operatorname{de} \mathcal{T}\left(\boldsymbol{R}_{>1}^{\Sigma(1) / G}\right)$, on a la représentation intégrale suivante

$$
\begin{aligned}
& \sum_{t \in T(K)} H(t,-s) \\
& =\frac{1}{\log (q)^{\operatorname{rg}\left(X(T)^{G}\right) b(T)}}\left(\frac{[A(T)]}{\left[\mathcal{K}_{T}\right]} I_{1}(\boldsymbol{s})+\sum_{\substack{\chi \in \mathcal{U}_{T} \\
\chi \notin \operatorname{Ker}\left(\gamma^{*}\right) \cdot\left(T\left(\boldsymbol{A}_{K}\right) / T\left(\boldsymbol{A}_{K}\right)^{1}\right)^{*}}} I_{\chi}(\boldsymbol{s})\right)
\end{aligned}
$$

où $I_{1}(\boldsymbol{s})$ est l'intégrale donnée par l'expression (5.85) et, pour $\chi \in \mathcal{U}_{T} \backslash$ $\operatorname{Ker}\left(\gamma^{*}\right) .\left(T\left(\boldsymbol{A}_{K}\right) / T\left(\boldsymbol{A}_{K}\right)^{1}\right)^{*}, I_{\chi}(\boldsymbol{s})$ est l'intégrale donnée par l'expression (5.86).

Démonstration : Soit $\boldsymbol{s}$ un élément de $\mathcal{T}\left(\mathbf{R}_{>1}^{\Sigma(1) / G}\right)$. On a vu (lemme $5.31)$ que $H(-\boldsymbol{s},$.$) est intégrable sur T\left(\mathbf{A}_{K}\right)$. D'après la remarque 4.21 , on peut alors appliquer le lemme 4.20. On obtient donc, compte tenu du lemme 5.49, l'égalité

$$
\sum_{t \in T(K)} H(t,-\boldsymbol{s})=\frac{1}{\log (q)^{\mathrm{rg}\left(X(T)^{G}\right)} b(T)} \sum_{\chi^{\prime} \in \mathcal{U}_{T}} \int_{\left(T\left(\mathbf{A}_{K}\right) / T\left(\mathbf{A}_{K}\right)^{1}\right)^{*}} \mathcal{F} H\left(\chi \chi^{\prime},-\boldsymbol{s}\right) \widetilde{d \chi} .
$$

On décompose la somme $\sum_{\chi^{\prime} \in \mathcal{U}_{T}}$ apparaissant dans le membre de droite de (5.88) (qui est, rappelons-le, une somme finie) suivant que $\chi^{\prime}$ appartient ou non à $\operatorname{Ker}\left(\gamma^{*}\right) .\left(T\left(\mathbf{A}_{K}\right) / T\left(\mathbf{A}_{K}\right)^{1}\right)^{*}$.

D'après le lemme 5.53 , on a

$$
\begin{gathered}
\sum_{\substack{\chi \in \mathcal{U}_{T} \\
\chi^{\prime} \in \operatorname{Ker}\left(\gamma^{*}\right) \cdot\left(T\left(\mathbf{A}_{K}\right) / T\left(\mathbf{A}_{K}\right)^{1}\right)^{*}}} \mathcal{F} H\left(\chi \cdot \chi^{\prime},-\boldsymbol{s}\right) \widetilde{d \chi} \\
=\frac{[A(T)]}{\left[\mathcal{K}_{T}\right]} \int_{\left(T\left(\mathbf{A}_{K}\right) / T\left(\mathbf{A}_{K}\right)^{1}\right)^{*}} \int_{\left.T\left(\mathbf{A}_{K}\right)^{1}\right)^{*}}\left[\int_{\frac{T(K)}{T(T)}\left(\mathbf{A}_{K}\right)} H(-\boldsymbol{s}, t) \chi(t) \omega_{T}(t)\right] \widetilde{d \chi}
\end{gathered}
$$


D'après le corollaire 5.52, on a

$$
\begin{gathered}
\int_{\left(T\left(\mathbf{A}_{K}\right) / T\left(\mathbf{A}_{K}\right)^{1}\right)^{*}}\left[\int_{\overline{T(K)} \cap T\left(\mathbf{A}_{K}\right)} H(-\boldsymbol{s}, t) \chi(t) \omega_{T}(t)\right] \widetilde{d \chi} \\
=\int_{X(T) G}^{G}\left[\int_{\frac{G}{T(K)} \cap T\left(\mathbf{A}_{K}\right)} H(-\boldsymbol{s}, t) \chi_{\boldsymbol{z}}(t) \omega_{T}(t)\right] d \boldsymbol{z} .
\end{gathered}
$$

Toujours d'après le corollaire 5.52, pour $\chi^{\prime} \in \mathcal{U}_{T} \backslash \operatorname{Ker}\left(\gamma^{*}\right) .\left(T\left(\mathbf{A}_{K}\right) / T\left(\mathbf{A}_{K}\right)^{1}\right)^{*}$, on a

$$
\int_{\left(T\left(\mathbf{A}_{K}\right) / T\left(\mathbf{A}_{K}\right)^{1}\right)^{*}} \mathcal{F} H\left(\chi \chi^{\prime},-\boldsymbol{s}\right) \widetilde{d \chi}=\int_{X(T)_{U}^{G}} \mathcal{F} H\left(\chi_{\boldsymbol{z}} \chi^{\prime},-\boldsymbol{s}\right) d \boldsymbol{z} .
$$

On en déduit le résultat annoncé.

Le cas où l'hypothèse (4.8) est vérifiée pour toute place. Si toutes les places $v$ de $K$ vérifient l'hypothèse (4.8), d'après les lemmes 5.38 et 5.40 on a pour tout $\boldsymbol{s} \in \mathcal{T}\left(\mathbf{R}_{>1}^{\Sigma(1) / G}\right)$ et tout $\chi \in\left(T\left(\mathbf{A}_{K}\right) / \mathbf{K}(T)\right)^{*}$

$$
I_{1}(\boldsymbol{s})=\int_{X(T)_{U}^{G}}\left(\prod_{\alpha \in \Sigma(1) / G} Z_{K_{\alpha}}\left(\left\langle\boldsymbol{z}, d_{\alpha} \rho_{\alpha}\right\rangle q^{-d_{\alpha} s_{\alpha}}\right)\right) \mathfrak{f}\left(\gamma_{\boldsymbol{U}}(\boldsymbol{z}) q^{-\boldsymbol{s}}\right) d \boldsymbol{z} .
$$

et

$$
I_{\chi}(\boldsymbol{s})=\int_{X(T)_{U}^{G}}\left(\prod_{\alpha \in \Sigma(1) / G} \mathcal{L}_{K_{\alpha}}\left(\chi_{\alpha},\left\langle\boldsymbol{z}, d_{\alpha} \rho_{\alpha}\right\rangle q^{-d_{\alpha} s_{\alpha}}\right)\right) \mathfrak{f}\left(\chi, \gamma_{\boldsymbol{U}}(\boldsymbol{z}) q^{-\boldsymbol{s}}\right) d \boldsymbol{z}
$$

La technique pour évaluer de telles intégrales est développée à la section 7 .

Le cas d'une extension de déploiement non ramifiée. Le but de ce paragraphe est de décrire une situation techniquement plus simple que la situation générale dans le cas fonctionnel, pour laquelle un lemme technique simplifié sera suffisant. La compréhension préalable de ce qui se passe dans ce cas peut aider à la compréhension du traitement du cas général. 


\section{Corollaire 5.55}

On se place dans le cas fonctionnel et on suppose que l'extension de déploiement $L / K$ est non ramifiée. Pour tout élément de $\boldsymbol{s}$ de $\mathcal{T}\left(\boldsymbol{R}_{>1}^{\Sigma(1) / G}\right)$, on a la relation

$$
\sum_{t \in T(K)} H(t,-s)=\frac{1}{\log (q)^{\operatorname{rg}\left(X(T)^{G}\right)} b(T)} \sum_{\chi \in \mathcal{U}_{T}} I_{\chi}(\boldsymbol{s})
$$

où, pour $\chi \in \mathcal{U}_{T}, I_{\chi}(s)$ est l'intégrale donnée par

$$
I_{\chi}(\boldsymbol{s})=\int_{X(T)_{U}^{G}}\left(\prod_{\alpha \in \Sigma(1) / G} \mathcal{L}_{K_{\alpha}}\left(\chi_{\alpha},\left\langle\boldsymbol{z}, d_{\alpha} \rho_{\alpha}\right\rangle q^{-d_{\alpha} s_{\alpha}}\right)\right) \mathfrak{Q}\left(\chi, \gamma_{\boldsymbol{U}}(\boldsymbol{z}) q^{-\boldsymbol{s}}\right) d \boldsymbol{z} \text {. }
$$

Démonstration : D'après (5.88) et le corollaire 5.52 on a pour tout $s \in$ $\mathcal{T}\left(\mathbf{R}_{>1}^{\Sigma(1) / G}\right)$

$$
\sum_{t \in T(K)} H(t,-\boldsymbol{s})=\frac{1}{\log (q)^{\operatorname{rg}\left(X(T)^{G}\right)} b(T)} \sum_{\chi \in \mathcal{U}_{T}} \int_{X(T)_{U}^{G}} \mathcal{F} H\left(\chi \chi_{\boldsymbol{z}},-\boldsymbol{s}\right) d \boldsymbol{z} .
$$

D'après le lemme 5.38 , on a pour tout $\chi \in \mathcal{U}_{T}$

$$
\mathcal{F} H\left(\chi \cdot \chi_{\boldsymbol{z}},-\boldsymbol{s}\right)=\left(\prod_{\alpha \in \Sigma(1) / G} \mathcal{L}_{K_{\alpha}}\left(\chi_{\alpha},\left\langle\boldsymbol{z}, d_{\alpha} \rho_{\alpha}\right\rangle q^{-d_{\alpha} s_{\alpha}}\right)\right) \mathfrak{f}\left(\chi, \gamma_{\boldsymbol{U}}(\boldsymbol{z}) q^{-\boldsymbol{s}}\right)
$$

Comme la variété $X_{\Sigma}$ est déployée par une extension non ramifiée, d'après la définition $(5.16)$ de $\mathfrak{f}(\chi,$.$) on a \mathfrak{f}(\chi,)=.\mathfrak{Q}(\chi,$.$) d'où le résultat.$

\section{6 Évaluation de l'intégrale : le cas arithmétique}

Soit $N$ un Z-module libre de rang fini et $\Lambda$ un cône strictement convexe de $N_{\mathbf{R}}$. On définit, pour tout élément $s$ de $\mathcal{T}(\operatorname{intrel}(\Lambda)$,

$$
\Xi_{N, \Lambda}(\boldsymbol{s})=\int_{\Lambda^{\vee}} e^{-\langle y, \boldsymbol{s}\rangle} d y
$$

où $d y$ est la mesure de Lebesgue sur $N_{\mathbf{R}}^{\vee}$, normalisée de sorte que le réseau $N^{\vee}$ soit de covolume 1 . Cette fonction sera appelée $\Xi$-fonction du cône $\Lambda$. Notons que si $\boldsymbol{s}_{0}$ est un élément de $\mathcal{T}(\operatorname{intrel}(\Lambda))$ on a

$$
\Xi_{N, \Lambda}\left(\boldsymbol{s}_{0}\right)=\lim _{t \rightarrow 0} t^{\mathrm{rg}(N)} \Xi_{N, \Lambda}\left(t \boldsymbol{s}_{0}\right)
$$


et que si $N^{\prime} \subset N$ est un sous-groupe d'indice fini on a

$$
\Xi_{N, \Lambda}(s)=\left[N: N^{\prime}\right] \Xi_{N^{\prime}, \Lambda}(s) .
$$

Remarque 6.1: D'après (2.11), on a en particulier, pour toute variété projective et lisse $V$ telle que la classe du faisceau anticanonique appartient à l'intérieur du cône effectif de $V$

$$
\alpha^{*}(V)=\Xi_{\operatorname{Pic}\left(X_{\Sigma}\right), C_{\text {eff }}(V)}\left(\omega_{V}^{-1}\right)
$$

où $C_{\text {eff }}(V)$ est le cône effectif de $V$.

L'évaluation est basée sur le résultat suivant, cas particulier de [CLTs, Thm 3.1.14].

\section{Théorème 6.2}

Soit $n \geqslant 1$ un entier et $\Gamma$ un sous-groupe de $\boldsymbol{Z}^{n}$ tel que $N=Z^{n} / \Gamma$ est sans torsion et $\Gamma \cap \boldsymbol{R}_{\geqslant 0}^{n}=\{0\}$. On note $j$ le morphisme quotient $\boldsymbol{Z}^{n} \rightarrow N$.

Soit $f$ une fonction holomorphe sur $\mathcal{T}\left(\boldsymbol{R}_{>0}^{n}\right)$. On suppose qu'il existe un $\varepsilon>0$ tel que la fonction

$$
s \mapsto f(s) \prod_{1 \leqslant i \leqslant n} \frac{s_{i}}{1+s_{i}}
$$

se prolonge en une fonction holomorphe sur $\mathcal{T}\left(\boldsymbol{R}_{>-\varepsilon}^{n}\right)$ qui est $\Gamma_{\boldsymbol{R}^{-c o n t r o ̂ l e ́ e ~}}$ au sens de [CLTs, Définition 3.13]. On note $C$ la valeur de ce prolongement en 0 .

Alors l'intégrale

$$
\frac{1}{(2 \pi)^{\operatorname{rg}(\Gamma)}} \int_{y \in \Gamma_{\boldsymbol{R}}} f(\boldsymbol{s}+i y) d y
$$

converge absolument en tout $\boldsymbol{s}$ de $\mathcal{T}\left(\boldsymbol{R}_{>0}^{n}\right)$ et définit une fonction holomorphe

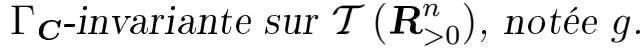

Il existe en outre un $\varepsilon^{\prime}>0$ tel que la fonction

$$
R: s \mapsto g(s)-C \Xi_{N, j\left(\boldsymbol{R}_{\geqslant 0}^{n}\right)}(j(s))
$$

se prolonge en une fonction méromorphe sur $\mathcal{T}\left(\boldsymbol{R}_{>-\varepsilon^{\prime}}^{n}\right)$

Pour tout $\boldsymbol{s}_{0} \in \mathcal{T}\left(\boldsymbol{R}_{>0}^{n}\right)$, on a

$$
\lim _{s \rightarrow 0} s^{\operatorname{rg}(N)} R\left(s \boldsymbol{s}_{0}\right)=0 .
$$

Remarques 6.3: 
1. La $\Gamma_{\mathbf{R}}$-contrôlabilité de $f$ signifie en deux mots que l'on dispose d'un bon contrôle de la croissance de $f$ sur les bandes en $\Gamma_{\mathbf{R}}$. Nous n'avons pas estimé utile de rappeler ici la définition précise de cette notion. Il suffit de savoir que, pour l'application du théorème à l'évaluation du comportement asymptotique de la fonction zêta des hauteurs, cette hypothèse est vérifiée grâce au point 3 de la proposition 5.46 , comme déjà signalé à la remarque 5.47 .

2. Le résultat obtenu en appliquant [CLTs, Thm 3.1.14] est en fait plus précis. On obtient une description des pôles de $g$ au voisinage de zéro, et un contrôle de $g$ dans les bandes verticales. Une fois le résultat appliqué à la fonction zêta des hauteurs, ce contrôle joint à un théorème taubérien adéquat permet de donner un développement asymptotique du nombre de points de hauteur bornée plus précis que celui qui découle du théorème 4.12 .

3. L'idée générale de la preuve de [CLTs, Thm 3.1.14] est de mettre en oeuvre une récurrence sur le rang de $\Gamma$ utilisant le théorème des résidus. La notion de contrôlabilité sert à assurer l'intégrabilité (et le contrôle dans les bandes) des fonctions obtenues par intégrations successives. Cette preuve nous semble inadaptable telle quelle au type de fonctions que l'on a à traiter dans le cas fonctionnel. Une des raisons est l'apparition de pôles supplémentaires. En outre, ce que devrait être la définition de la contrôlabilité dans ce cadre n'est pas clair a priori ; notamment, au vu de la périodicité des fonctions mises en jeu, la notion de majoration dans les bandes perd tout son intérêt.

\section{7 Évaluation de l'intégrale : le cas fonctionnel}

Le but de cette partie est d'obtenir un résultat analogue au théorème 6.2 , adapté à la forme des fonctions obtenues dans le cas fonctionnel.

\subsection{Définition d'une certaine classe de fonctions}

Soit $N$ un Z-module libre de rang fini. Pour toute partie $A$ de $N_{\mathbf{R}}$ et toute application $a: A \cap N \rightarrow \mathbf{C}$ on définit la série formelle

$$
\mathcal{L}_{N, A, a}(T)=\sum_{y \in A \cap N} a_{y} T^{y} .
$$


On pose

$$
\mathcal{L}_{N, A, a}(\boldsymbol{z})=\sum_{y \in A \cap N} a_{y}\langle\boldsymbol{z}, y\rangle
$$

pour tout élément $\boldsymbol{z}$ de $N_{\mathbf{C}^{\times}}^{\vee}$ telle que le membre de droite de (7.2) est une série absolument convergente. On a ainsi

$$
\mathcal{L}_{N, A, a}\left(q^{-\boldsymbol{s}}\right)=\sum_{y \in A \cap N} a_{y} q^{-\langle y, s\rangle}
$$

pour tout élément $s$ de $N_{\mathbf{C}}^{\vee}$ tel que telle que le membre de droite de (7.3) est une série absolument convergente. Pour un tel $s$ on a donc, pour tout élément $\boldsymbol{z}$ de $N_{\boldsymbol{U}}^{\vee}$

$$
\mathcal{L}_{N, A, a}\left(\boldsymbol{z} q^{-\boldsymbol{s}}\right)=\sum_{y \in A \cap N} a_{y}\langle\boldsymbol{z}, y\rangle q^{-\langle y, \boldsymbol{s}\rangle} .
$$

Si $a$ est la fonction constante égale à 1 , on notera $\mathcal{L}_{N, A}$ pour $\mathcal{L}_{N, A, a}$.

\subsection{Un premier exemple}

Soit $\Upsilon$ un cône de $N_{\mathbf{R}}$, tel que $\Upsilon^{\vee}$ soit d'intérieur non vide. La série définissant $\mathcal{L}_{N, \Upsilon}\left(q^{-s}\right)$ converge alors absolument pour tout $s$ de $\mathcal{T}\left(\operatorname{int}\left(\Upsilon^{\vee}\right)\right.$ ). La fonction $\mathcal{L}_{N, \Upsilon}\left(q^{-\boldsymbol{s}}\right)$ est utilisée par Peyre dans [Pe3] pour une définition alternative de l'invariant $\alpha^{*}(V)$ attaché à une variété $V$ (cf. la formule (2.11) pour la définition adoptée dans ce texte). On a en effet le résultat suivant :

\section{Lemme 7.1}

Soit $\lambda_{0}$ un élément de l'intérieur de $\Upsilon^{\vee}$. L'application

$$
s \longmapsto \mathcal{L}_{N, \Upsilon}\left(q^{-s \cdot \lambda_{0}}\right)
$$

est bien définie et holomorphe sur $\mathcal{T}\left(\boldsymbol{R}_{>0}\right)$, et se prolonge en une fonction méromorphe sur $\boldsymbol{C}$, avec un pôle d'ordre au plus la dimension de $\Upsilon$ en $s=0$. Si de plus $\Upsilon^{\vee}$ est strictement convexe, l'ordre de ce pôle est exactement $\operatorname{rg}(N)$, et on a

$$
\lim _{s \rightarrow 0}\left(s^{\operatorname{rg}(N)} \mathcal{L}_{N, \Upsilon}\left(q^{-s \cdot \lambda_{0}}\right)\right)=\log (q)^{-\operatorname{rg}(N)} \Xi_{N, \Upsilon \vee}\left(\lambda_{0}\right) .
$$

Remarque 7.2: On a donc en particulier d'après (6.4)

$$
\alpha^{*}\left(X_{\Sigma}\right)=\log (q)^{\operatorname{rg}\left(\operatorname{Pic}\left(X_{\Sigma}\right)\right)} \lim _{s \rightarrow 0}\left[s^{\operatorname{rg}\left(\operatorname{Pic}\left(X_{\Sigma}\right)\right)} \mathcal{L}_{\operatorname{Pic}\left(X_{\Sigma}\right)^{\vee}, C_{\text {eff }}\left(X_{\Sigma}\right)^{\vee}}\left(q^{-s\left[-\mathcal{K}_{X_{\Sigma}}\right]}\right)\right] .
$$


Remarque 7.3: Si $N^{\prime} \subset N$ est un sous-groupe d'indice fini de $N$ on a

$$
\lim _{s \rightarrow 0}\left[s^{\operatorname{rg}(N)} \mathcal{L}_{N, \Upsilon}\left(q^{-s \lambda_{0}}\right)\right]=\left[N: N^{\prime}\right] \lim _{s \rightarrow 0}\left[s^{\operatorname{rg}(N)} \mathcal{L}_{N^{\prime}, \Upsilon}\left(q^{-s \lambda_{0}}\right)\right] .
$$

Démonstration : On écrit $\Upsilon$ comme le support d'un éventail régulier $\Delta$. Concernant cet éventail, on reprend les notations introduites à la section 4.1.

On a alors

$$
\mathcal{L}_{N, \Upsilon}(T)=\sum_{\delta \in \Delta} L_{N, N \cap \operatorname{intrel}(\delta)}(T),
$$

soit encore en termes plus explicites

$$
\mathcal{L}_{N, \Upsilon}(T)=\sum_{\delta \in \Delta} \prod_{l \in \delta(1)}\left(\frac{1}{1-T^{\rho_{l}}}-1\right) .
$$

On a donc

$$
\mathcal{L}_{N, \Upsilon}\left(q^{-\boldsymbol{s}}\right)=\sum_{\delta \in \Delta} \prod_{l \in \delta(1)}\left(\frac{1}{1-q^{-\left\langle\rho_{l}, \boldsymbol{s}\right\rangle}}-1\right),
$$

d'où le résultat, car le cardinal maximal des ensembles $\delta(1)$ est égal à la dimension de $\Upsilon$.

Supposons à présent $\Upsilon$ de dimension $\operatorname{rg}(N)$, et montrons la dernière assertion du lemme. Comme les cônes de dimension maximale de $\Delta$ recouvrent $\Upsilon$ et que leurs intersections sont de mesure de Lebesgue nulle, on peut écrire

$$
\begin{aligned}
& \Xi_{N, \Upsilon \vee}\left(\lambda_{0}\right)=\int_{\Upsilon} e^{-\left\langle y, \lambda_{0}\right\rangle} d y, \\
& =\sum_{\substack{\delta \in \Delta \\
\operatorname{dim}(\delta)=\operatorname{rg}(N)}} \int_{\delta} e^{-\left\langle y, \lambda_{0}\right\rangle} d y,
\end{aligned}
$$

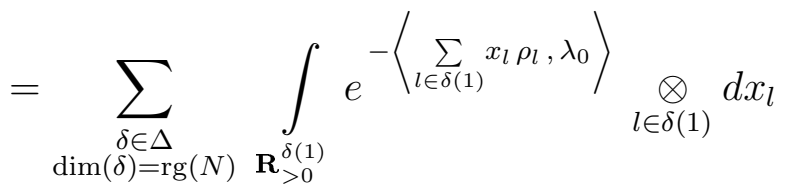

$$
\begin{aligned}
& =\sum_{\substack{\delta \in \Delta \\
\operatorname{dim}(\delta)=\operatorname{rg}(N)}} \prod_{l \in \delta(1)} \frac{1}{\left\langle\rho_{l}, \lambda_{0}\right\rangle} .
\end{aligned}
$$

Or, d'après (7.7) et le fait que pour tout complexe non nul $z$ on a

$$
\lim _{s \rightarrow 0} \frac{s}{1-q^{s z}}=-\frac{1}{\log (q) z}
$$


on obtient

$$
\lim _{s \rightarrow 0} s^{\operatorname{rg}(N)} \mathcal{L}_{N, \Upsilon}\left(q^{-s \lambda_{0}}\right)=\sum_{\substack{\delta \in \Delta \\ \operatorname{dim}(\delta)=\operatorname{rg}(N)}} \log (q)^{-\operatorname{rg}(N)} \prod_{l \in \delta(1)} \frac{1}{\left\langle\rho_{l}, \lambda_{0}\right\rangle},
$$

d'où le résultat.

\subsection{Encore quelques définitions}

On fixe désormais pour toute la suite de la section 7 une base $\left(\lambda_{i}\right)_{i \in I}$ de $N$. On note $\left(\lambda_{i}^{\vee}\right)_{i \in I}$ la base duale d'une telle base, $\Lambda$ le cône simplicial de $N_{\mathbf{R}}$ engendré par cette base et

$$
\lambda^{\vee}=\sum_{i \in I} \lambda_{i}^{\vee}
$$

On définit, pour tout réel $\eta$,

$$
\Lambda_{>\eta}^{\vee}=\sum_{i \in I} \mathbf{R}_{>\eta} \lambda_{i}^{\vee} \subset N_{\mathbf{R}}^{\vee}
$$

Ainsi $\Lambda_{>0}^{\vee}$ est l'intérieur de $\Lambda^{\vee}$.

Soit

$$
a: \Lambda \cap N \rightarrow \mathbf{C}
$$

une application et $\varepsilon>0$ un réel tels que la série définissant $\mathcal{L}_{N, \Lambda, a}\left(q^{-\boldsymbol{s}}\right)$ converge absolument pour tout $s$ du domaine $\mathcal{T}\left(\Lambda_{>-\varepsilon}^{\vee}\right)$. Ceci équivaut à demander la convergence de la série

$$
\sum_{y \in \Lambda \cap N}\left|a_{y}\right| q^{\eta\left\langle y, \lambda^{\vee}\right\rangle}
$$

pour tout $\eta<\varepsilon$.

La fonction

$$
\boldsymbol{s} \mapsto \mathcal{L}_{N, \Lambda, a}\left(q^{-s}\right) .
$$

est donc holomorphe sur le domaine $\mathcal{T}\left(\Lambda_{>-\varepsilon}^{\vee}\right)$. Une telle fonction sera appelée fonction admissible élémentaire de multiplicité supérieure à zéro.

Si $r \geqslant 1$ est un entier, une fonction $f$ holomorphe sur $\mathcal{T}\left(\Lambda_{>0}^{\vee}\right)$ sera appelée fonction admissible élémentaire de multiplicité supérieure $\grave{a}-r$ s'il existe une écriture

$$
f(s)=g(s) \mathcal{L}_{N^{\prime}, \operatorname{intrel}(\Upsilon)}\left(q^{-s}\right)
$$

où $\Upsilon \subset \Lambda$ est un cône de dimension inférieure à $r, N^{\prime}$ est un sous-groupe de $N$ et $g$ est admissible élémentaire de multiplicité supérieure à 0 . Une telle 
fonction $f$ se prolonge donc en une fonction méromorphe sur le domaine $\mathcal{T}\left(\Lambda_{>-\varepsilon}^{\vee}\right)$ pour un certain $\varepsilon>0$.

Si $r \geqslant 0$ est un entier, on appellera fonction admissible de multiplicité supérieure $\grave{a}-r$ une fonction $f$ holomorphe sur le domaine $\mathcal{T}\left(\Lambda_{>0}^{\vee}\right)$ qui s'écrit comme une somme finie de fonction admissibles élémentaires de multiplicité supérieure à $-r$.

Une telle fonction $f$ se prolonge donc en une fonction méromorphe sur le domaine $\mathcal{T}\left(\Lambda_{>-\varepsilon}^{\vee}\right)$ pour un certain $\varepsilon>0$. Par ailleurs, pour tout $\lambda_{0}^{\vee}$ élément de l'intérieur de $\Lambda^{\vee}$, la fonction d'une variable complexe

$$
s \mapsto f\left(s \lambda_{0}^{\vee}\right)
$$

est méromorphe sur un voisinage de zéro, et a un pôle d'ordre au plus $r$ en zéro.

\subsection{Avertissement au lecteur}

Nous allons donner ci-dessous trois versions du lemme technique d'intégration destiné à évaluer le comportement analytique de la fonction zêta des hauteurs à partir de la représentation intégrale obtenue à la sous-section 5.6.2. Ces trois versions seront de généralité (et de difficulté technique) croissante. Le parti pris de ne pas présenter directement la version la plus générale nous semble utile pour la compréhension de la technique employée.

La première version (lemme 7.8) est une version-jouet, très simple et transparente. Elle est destinée à faire comprendre l'idée élémentaire de base qui sous-tend les versions plus élaborées qui vont suivre, mais ne nous servira pas pour l'évaluation de la fonction zêta des hauteurs.

La deuxième version (lemme 7.10) est une généralisation naturelle de la première, et est suffisante pour traiter le cas des variétés toriques déployées par une extension non ramifiée. Elle s'appuie sur un «lemme de décomposition» de certaines fonctions caractéristiques «tordues» associées à des cônes (lemme 7.4).

La troisième version (lemmes 7.12 et 7.14), la plus générale, est nécessaire pour traiter le cas d'une extension de déploiement quelconque, et présente quelques complications techniques qui peuvent la rendre un peu obscure au premier abord. Elle s'appuie sur des généralisations du lemme de décomposition 7.4 (lemmes 7.5 et 7.6 )

Nous conseillons donc de ne pas aborder en première lecture les démonstrations des lemmes 7.5, 7.6 7.12 et 7.14 (i.e. les sous-sections 7.5.2 et 7.6.3), ainsi que l'application qui en est faite à l'évaluation du comportement analytique de la fonction zêta des hauteurs des variétés toriques dans le cas général (sous-section 8.3.2). 


\subsection{Un lemme de décomposition}

\subsubsection{Version simple}

Soit $\Upsilon$ un cône de $N_{\mathbf{R}}$ contenu dans $\Lambda$. On notera $\langle\Upsilon\rangle$ le sous-espace vectoriel de $N_{\mathbf{R}}$ engendré par $\Upsilon$.

On écrit $\Upsilon$ comme le support d'un éventail régulier $\Delta$. Concernant cet éventail, on reprend les notations introduites à la section 4.1. Pour $l \in \Delta(1)$, $\rho_{l}$ désigne donc le générateur du monoïde $N \cap l$. Pour toute partie $I$ de $\Delta(1)$ nous noterons $\mathcal{C}(I)$ le cône engendré par les $\left(\rho_{i}\right)_{i \in I}$.

On fixe un élément $z$ de $\Lambda \cap N$. On pose

$$
\Upsilon_{z}=\Upsilon \cap\{z+\Lambda \cap N\}
$$

On veut étudier la série formelle

$$
\mathcal{L}_{N, \Upsilon_{z}}(T)=\sum_{\substack{y \in \Upsilon \cap N \\ y \in z+\Lambda \cap N}} T^{y}
$$

Nous utilisons la même technique que dans la section 4.3.3. de [Bo2]. Nous écrivons d'abord

$$
\mathcal{L}_{N, \Upsilon_{z}}(T)=\sum_{\delta \in \Delta} \mathcal{L}_{N, \Upsilon_{z} \cap \operatorname{intrel}(\delta)}(T) .
$$

Pour $\delta \in \Delta$, notons qu'on peut écrire

$$
\mathcal{L}_{N, \Upsilon_{z} \cap \operatorname{intrel}(\delta)}(T)=\sum_{\substack{y \in \operatorname{intrel}(\delta) \\ y-z \in \Lambda}} T^{y}=\sum_{\substack{y \in \operatorname{intrel}(\delta) \\ \forall i \in I,\left\langle\lambda_{i}^{\vee}, y\right\rangle \geqslant\left\langle\lambda_{i}^{\vee}, z\right\rangle}} T^{y} .
$$

Pour tout sous-ensemble $K$ de $I$ et tout cône $C$ de $N_{\mathbf{R}}$, on note $C(K, z)$ le sous-ensemble de intrel $(C)$ formé des éléments $y$ vérifiant la condition

$$
\forall i \in K,\left\langle\lambda_{i}^{\vee}, y\right\rangle<\left\langle\lambda_{i}^{\vee}, z\right\rangle .
$$

En utilisant (7.4), on voit alors qu'on a une décomposition

$$
\mathcal{L}_{N, \Upsilon_{z} \cap \operatorname{intrel}(\delta)}(T)=\sum_{K \subset I}(-1)^{[K]} \mathcal{L}_{N, \delta(K, z)}(T) .
$$

Posons

$$
\mathcal{M}=[I] \operatorname{Sup}_{l \in \Delta(1)}\left\langle\rho_{l}, \lambda\right\rangle
$$


Lemme 7.4

Soient $\delta$ un cône de $\Delta$, et $K$ une partie de $I$. Soit $\delta(1)_{K}$ le sous-ensemble de $\delta(1)$ donné par

$$
\delta(1)_{K}=\left\{l \in \delta(1), \forall i \in K, \quad\left\langle\rho_{l}, \lambda_{i}\right\rangle=0\right\}
$$

et $F(\delta, K, z)$ le sous-ensemble de $\delta(K, z)$ donné par

$$
F(\delta, K, z)=\mathcal{C}\left(\delta(1) \backslash \delta(1)_{K}\right)(K, z) .
$$

Alors $\delta(1)_{K}$ et $F(\delta, K, z)$ vérifient les propriétés suivantes :

- On a une écriture

$$
\mathcal{L}_{N, \delta(K, z)}(T)=\mathcal{L}_{N, \text { intrel }\left(\mathcal{e}\left(\delta(1)_{K}\right)\right)}(T) \mathcal{L}_{N, F(\delta, K, z)}(T)
$$

- $F(\delta, K, z)$ est fini. Plus précisément on a la majoration

$$
[F(\delta, K, z)] \leqslant\langle z, \lambda\rangle^{\operatorname{rg}(N)} .
$$

- Pour tout $y \in F(\delta, K, z)$ on a

$$
\langle y, \lambda\rangle \leqslant \mathcal{M}\langle z, \lambda\rangle .
$$

- Si $K$ est vide, on a $\delta(1)_{K}=\delta(1)$ et $F(\delta, K, z)=\varnothing$.

- On suppose en outre que $(N /\langle\Upsilon\rangle)^{\vee} \cap \Lambda^{\vee}=\{0\}$, que $\delta$ est de dimension maximale, et que $K$ est non vide. Alors $\delta(1)_{K}$ est un sous-ensemble strict de $\delta(1)$.

Démonstration : Cette démonstration est très similaire à celle du lemme 3 de [Bo2].

Soit $y$ un élément de intrel $(\delta)$. Il s'écrit donc de manière unique $y_{1}+y_{2}$ avec $y_{1} \in \operatorname{intrel}\left(\mathcal{C}\left(\delta(1)_{K}\right)\right)$ et $y_{2} \in \operatorname{intrel}\left(\mathcal{C}\left(\delta(1) \backslash \delta(1)_{K}\right)\right)$.

Au vu de la définition $(7.8)$ de $\delta(1)_{K}$, on a

$$
\forall i \in K, \quad\left\langle y, \lambda_{i}\right\rangle=\left\langle y_{2}, \lambda_{i}\right\rangle
$$

Supposons en outre que $y$ est dans $\delta(K, z)$, i.e. vérifie

$$
\forall i \in K, \quad\left\langle y, \lambda_{i}^{\vee}\right\rangle\left\langle\left\langle z, \lambda_{i}^{\vee}\right\rangle .\right.
$$

On a donc

$$
\forall i \in K, \quad\left\langle y_{2}, \lambda_{i}^{\vee}\right\rangle<\left\langle z, \lambda_{i}^{\vee}\right\rangle,
$$

en d'autres termes $y_{2}$ est dans $\mathcal{C}\left(\delta(1) \backslash \delta(1)_{K}, K, z\right)=F(\delta, K, z)$. 
Réciproquement, on constate que si on a

$$
y_{1} \in \operatorname{intrel}\left(\mathcal{C}\left(\delta(1)_{K}\right)\right)
$$

et

$$
y_{2} \in \mathcal{C}\left(\delta(1) \backslash \delta(1)_{K}, K, z\right)=F(\delta, K, z)
$$

alors $y_{1}+y_{2}$ est un élément de $\delta(K, z)$.

Ceci montre qu'on a une décomposition

$$
\mathcal{L}_{N, \delta(K, z)}(T)=\mathcal{L}_{N, \operatorname{intrel}\left(e\left(\delta(1)_{K}\right)\right)}(T) \mathcal{L}_{N, F(\delta, K, z)}(T) .
$$

Montrons que $F(\delta, K, z)$ est fini et majorons son cardinal. Soit $y_{2}$ un élément de $F(\delta, K, z)$, qu'on écrit

$$
y_{2}=\sum_{l \in \delta(1) \backslash \delta(1)_{K}} \mu_{l} \rho_{l}
$$

avec les $\mu_{l}$ dans $\mathbf{N}_{>0}$.

Par définition de $\delta(1)_{K}$, pour tout $l$ de $\delta(1) \backslash \delta(1)_{K}$, il existe $i$ dans $K$ vérifiant $\left\langle\rho_{l}, \lambda_{i}\right\rangle \geqslant 1$ (rappelons que pour tout $i$ on a $\left\langle\rho_{l}, \lambda_{i}\right\rangle \geqslant 0$ ). Comme $y_{2}$ vérifie

$$
\forall i \in K, \quad\left\langle y_{2}, \lambda_{i}\right\rangle<\left\langle z, \lambda_{i}\right\rangle
$$

on a

$$
\mu_{l}<\operatorname{Sup}_{i \in K}\left\langle z, \lambda_{i}\right\rangle \leqslant\langle z, \lambda\rangle .
$$

Ainsi $F(\delta, K, z)$ est fini et son cardinal est majoré par

$$
\langle z, \lambda\rangle^{\left[\delta(1) \backslash \delta(1)_{K}\right]} \leqslant\langle z, \lambda\rangle^{\operatorname{rg}(N)} .
$$

Par ailleurs un $y_{2}$ de $F(\delta, K, z)$ vérifie

$$
0 \leqslant\left\langle y_{2}, \lambda\right\rangle \leqslant \sum_{l \in \delta(1) \backslash \delta(1)_{K}}\langle z, \lambda\rangle\left\langle\rho_{l}, \lambda\right\rangle \leqslant M\langle z, \lambda\rangle
$$

où on rappelle que

$$
\mathcal{M}=[I] \operatorname{Sup}_{l \in \Delta(1)}\left(\left\langle\rho_{l}, \lambda\right\rangle\right) .
$$

Supposons $(N /\langle\Upsilon\rangle)^{\vee} \cap \Lambda^{\vee}=\{0\}$. Ceci équivaut à dire que si $\lambda \in \Lambda^{\vee}$ vérifie $\langle m, \lambda\rangle=0$ pour tout $m \in\langle\Upsilon\rangle$ alors $\lambda=0$. Mais si $\delta$ est de dimension maximale, les $\left(\rho_{l}\right)_{l \in \delta(1)}$ engendrent $\langle\Upsilon\rangle$, et donc si $K$ n'est pas vide, on ne peut avoir $\delta(1)_{K}=\delta(1)$. 


\subsubsection{Version générale}

On considère toujours $\Upsilon$ un cône de $N_{\mathbf{R}}$ contenu dans $\Lambda$. On se donne en outre $N^{\prime} \subset N$ un sous-groupe d'indice fini.

On écrit cette fois $\Upsilon$ comme le support d'un éventail $N^{\prime}$-régulier $\Delta$ (i.e les cônes de $\Delta$ sont engendrés par des parties de bases de $N^{\prime}$ ), et on reprend à cet effet les notations introduites à la section 4.1. Soulignons que pour $l \in \Delta(1)$, $\rho_{l}$ désigne le générateur du monoïde $N^{\prime} \cap l$.

On fixe un élément $z$ de $\Lambda \cap N$. On pose

$$
\Upsilon_{z}=\Upsilon \cap\left\{z+\Lambda \cap N^{\prime}\right\}
$$

On veut étudier la série formelle

$$
\mathcal{L}_{N, \Upsilon_{z}}(T)=\sum_{\substack{y \in \Upsilon \cap N \\ y \in z+\Lambda \cap N^{\prime}}} T^{y} .
$$

Nous écrivons d'abord

$$
\mathcal{L}_{N, \Upsilon_{z}}(T)=\sum_{\delta \in \Delta} \mathcal{L}_{N, \Upsilon_{z} \cap \operatorname{intrel}(\delta)}(T)
$$

Pour $\delta \in \Delta$, notons qu'on peut écrire

$$
\mathcal{L}_{N, \Upsilon_{z} \cap \operatorname{intrel}(\delta)}(T)=\sum_{\substack{y \in \operatorname{intrel}(\delta) \\ y-z \in \Lambda \\ y-z \in N^{\prime}}} T^{y}=\sum_{\substack{y \in \operatorname{intrel}(\delta) \\ \forall i \in I,\left\langle\lambda_{i}^{\vee}, y\right\rangle \geqslant\left\langle\lambda_{i}^{\vee}, z\right\rangle \\ y-z \in N^{\prime}}} T^{y} .
$$

Pour tout sous-ensemble $K$ de $I$ et tout cône $C$ de $N_{\mathbf{R}}$, on note $C(K, z)$ le sous-ensemble de intrel $(C)$ formé des éléments $y$ vérifiant la condition

$$
\forall i \in K,\left\langle\lambda_{i}^{\vee}, y\right\rangle<\left\langle\lambda_{i}^{\vee}, z\right\rangle .
$$

Pour tout sous-ensemble $A$ de $N_{\mathbf{R}}$, on note $A_{z}$ l'ensemble des éléments $y$ de $A$ vérifiant la condition

$$
y-z \in N^{\prime}
$$

En utilisant (7.28), on voit alors qu'on a une décomposition

$$
\mathcal{L}_{N, \Upsilon_{z} \cap \operatorname{intrel}(\delta)}(T)=\sum_{K \subset I}(-1)^{[K]} \mathcal{L}_{N, \delta(K, z)_{z}}(T) .
$$

Posons

$$
\mathcal{M}=[I] \operatorname{Sup}_{l \in \Delta(1)}\left\langle\lambda^{\vee}, \rho_{l}\right\rangle
$$




\section{Lemme 7.5}

Soient $\delta$ un cône de $\Delta$, et $K$ une partie de $I$. Soit $\delta(1)_{K}$ le sous-ensemble de $\delta(1)$ défini par

$$
\delta(1)_{K}=\left\{l \in \delta(1), \forall i \in K, \quad\left\langle\lambda_{i}^{\vee}, \rho_{l}\right\rangle=0\right\} .
$$

On complète $\left(\rho_{l}\right)_{l \in \delta(1)}$ en une base $\left(\rho_{l}\right)_{l \in L}$ de $N^{\prime}$. Soit $N_{1}^{\prime}$ (respectivement $N_{2}^{\prime}$, respectivement $N_{3}^{\prime}$ ) le sous-groupe de $N$ engendré par les $\left(\rho_{l}\right)_{l \in \delta(1)_{K}}$ (respectivement $\left(\rho_{l}\right)_{l \in \delta(1) \backslash \delta(1)_{K}}$, respectivement $\left.\left(\rho_{l}\right)_{l \in L \backslash \delta(1)}\right)$.

On écrit

$$
z=z_{1}+z_{2}+z_{3}
$$

avec pour $i=1,2,3, z_{i} \in\left(N_{i}^{\prime}\right)_{\boldsymbol{Q}}$.

On note $z_{1}^{\prime}$ l'unique élément de $N^{\prime}$ vérifiant

$$
z_{1}^{\prime}-z_{1}=\sum_{l \in \delta(1)_{K}} \mu_{l} \rho_{l} \text { avec } 0 \leqslant \mu_{l}<1
$$

Soit $F(\delta, K, z)$ l'ensemble des éléments de $N$ qui s'écrivent

$$
y_{2}+z_{1}-z_{1}^{\prime}
$$

où $y_{2}$ est un élément de $\mathrm{C}\left(\delta(1) \backslash \delta(1)_{K}\right)(K, z)_{z_{2}}$.

Alors $\delta(1)_{K}, \delta(K, z)_{z}$ et $F(\delta, K, z)$ vérifient les propriétés suivantes.

1. Si $\delta(K, z)_{z}$ est non vide, alors $z_{3}$ est un élément de $N^{\prime}$.

2. Si $z_{3}$ est un élément de $N^{\prime}$, on a une décomposition

$$
\mathcal{L}_{N, \delta(K, z)_{z}}(T)=L_{N^{\prime}, \operatorname{intrel}\left(\mathcal{e}\left(\delta(1)_{K}\right)\right)}(T) \mathcal{L}_{N, F(\delta, K, z)}(T) .
$$

3. $F(\delta, K, z)$ est fini. Plus précisément, on a la majoration

$$
[F(\delta, K, z)] \leqslant\left(\left[N: N^{\prime}\right]\left\langle\lambda^{\vee}, z\right\rangle\right)^{\operatorname{rg}(N)} .
$$

4. Pour tout $y \in F(\delta, K, z)$ on a

$$
-\mathcal{M} \leqslant\left\langle\lambda^{\vee}, y\right\rangle \leqslant \mathcal{M}\left\langle\lambda^{\vee}, z\right\rangle .
$$

5. Si $K$ est vide, on a $\delta(1)_{K}=\delta(1)$ et $F(\delta, K, z)=\left\{z_{1}-z_{1}^{\prime}\right\}$.

6. On suppose en outre que $(N /\langle\Upsilon\rangle)^{\vee} \cap \Lambda^{\vee}=\{0\}$, que $\delta$ est de dimension maximale, et que $K$ est non vide. Alors $\delta(1)_{K}$ est un sous-ensemble strict de $\delta(1)$. 
Démonstration :

Soit $y$ un élément de intrel $(\delta)$. Il s'écrit donc de manière unique $y_{1}+y_{2}$ avec $y_{1} \in \operatorname{intrel}\left(\mathcal{C}\left(\delta(1)_{K}\right)\right)$ et $y_{2} \in \operatorname{intrel}\left(\mathcal{C}\left(\delta(1) \backslash \delta(1)_{K}\right)\right)$.

Supposons en outre que $y$ est dans $\delta(K, z)_{z}$, i.e. vérifie d'une part

$$
\forall i \in K, \quad\left\langle y, \lambda_{i}^{\vee}\right\rangle\left\langle\left\langle z, \lambda_{i}^{\vee}\right\rangle\right.
$$

et d'autre part

$$
y-z \in N^{\prime}
$$

Au vu de la définition (7.33) de $\delta(1)_{K}$, on a

$$
\forall i \in K, \quad\left\langle y, \lambda_{i}^{\vee}\right\rangle=\left\langle y_{2}, \lambda_{i}^{\vee}\right\rangle
$$

Ainsi la condition (7.40) montre que $y_{2}$ vérifie

$$
\forall i \in K, \quad\left\langle y_{2}, \lambda_{i}^{\vee}\right\rangle\left\langle\left\langle z, \lambda_{i}^{\vee}\right\rangle .\right.
$$

Par ailleurs on a $y-z=\left(y_{1}-z_{1}\right)+\left(y_{2}-z_{2}\right)+z_{3}$. En outre $y-z$ est dans $N^{\prime}$, et $y_{1}-z_{1}$ (respectivement $y_{2}-z_{2}$, respectivement $z_{3}$ ) est dans $\left(N_{1}^{\prime}\right)_{\mathbf{Q}}$ (respectivement $\left(N_{2}^{\prime}\right)_{\mathbf{Q}}$, respectivement $\left.\left(N_{3}^{\prime}\right)_{\mathbf{Q}}\right)$. On en déduit que $y_{1}-z_{1}$, $y_{2}-z_{2}$ et $z_{3}$ sont dans $N^{\prime}$.

Ainsi on a

$$
y_{2} \in \mathcal{C}\left(\delta(1) \backslash \delta(1)_{K}\right)(K, z)_{z_{2}}
$$

et

$$
y_{1} \in \operatorname{intrel}\left[\mathcal{C}\left(\delta(1)_{K}\right)\right]_{z_{1}} .
$$

En outre ceci montre que la non vacuité de $\delta(K, z)_{z}$ entraîne que $z_{3}$ est dans $N^{\prime}$.

Réciproquement, on constate que si on a

$$
\begin{gathered}
y_{1} \in \operatorname{intrel}\left[\mathcal{C}\left(\delta(1)_{K}\right)\right]_{z_{1}}, \\
y_{2} \in \mathcal{C}\left(\delta(1) \backslash \delta(1)_{K}\right)(K, z)_{z_{2}},
\end{gathered}
$$

et

$$
z_{3} \in N^{\prime}
$$

alors $y_{1}+y_{2}$ est un élément de $\delta(K, z)_{z}$.

Ceci montre que si $z_{3}$ est dans $N^{\prime}$ on a une décomposition

$$
\mathcal{L}_{N, \delta(K, z)}(T)=\left(\sum_{y_{2} \in \mathcal{C}\left(\delta(1) \backslash \delta(1)_{K}\right)(K, z)_{z_{2}}} T^{y_{2}}\right)\left(\sum_{y_{1} \in \operatorname{intrel}\left(\mathcal{e}\left(\delta(1)_{K}\right)\right)_{z_{1}}} T^{y_{1}}\right) .
$$


Par ailleurs on a

$$
\sum_{y_{1} \in \operatorname{intrel}\left(\mathcal{e}\left(\delta(1)_{K}\right)\right)_{z_{1}}} T^{y_{1}}=\sum_{\substack{y_{1} \in \operatorname{intrel}\left(\mathcal{C}\left(\delta(1)_{K}\right)\right) \\ y_{1}-z_{1} \in N^{\prime}}} T^{y_{1}}=T^{z_{1}-z_{1}^{\prime}} \sum_{\substack{y \in \operatorname{intrel}\left(\mathcal{C}\left(\delta(1)_{K}\right)\right)+z_{1}^{\prime}-z_{1} \\ y \in N^{\prime}}} T^{y}
$$

Or, au vu de la définition de $z_{1}^{\prime}$, on a

$$
\left[\operatorname{intrel}\left(\mathcal{C}\left(\delta(1)_{K}\right)\right)+z_{1}^{\prime}-z_{1}\right] \cap N^{\prime}=\operatorname{intrel}\left[\mathcal{C}\left(\delta(1)_{K}\right)\right] \cap N^{\prime}
$$

soit

$$
\sum_{\substack{y_{1} \in \operatorname{intrel}\left(\mathcal{C}\left(\delta(1)_{K}\right)\right) \\ y_{1}-z_{1} \in N^{\prime}}} T^{y_{1}}=T^{z_{1}-z_{1}^{\prime}} L_{N^{\prime}, \operatorname{intrel}\left(\mathcal{C}\left(\delta(1)_{K}\right)\right)}(T)
$$

d'où

$$
\begin{aligned}
\mathcal{L}_{N, \delta(K, z)}(T) & =\left(\sum_{y_{2} \in \mathcal{C}\left(\delta(1) \backslash \delta(1)_{K}\right)(K, z)_{z_{2}}} T^{y_{2}+z_{1}-z_{1}^{\prime}}\right) L_{N^{\prime}, \operatorname{intrel}\left(\mathrm{e}\left(\delta(1)_{K}\right)\right)}(T) \\
& =\mathcal{L}_{N, F(\delta, K, z)}(T) L_{N^{\prime}, \operatorname{intrel}\left(\mathcal{e}\left(\delta(1)_{K}\right)\right)}(T) .
\end{aligned}
$$

Montrons que $F(\delta, K, z)$ est fini et estimons son cardinal. On commence par remarquer que $F(\delta, K, z)$ est en bijection avec $\mathcal{C}\left(\delta(1) \backslash \delta(1)_{K}\right)\left(K, z_{2}\right)$. Soit $y_{2}$ un élément de $\mathcal{C}\left(\delta(1) \backslash \delta(1)_{K}\right)\left(K, z_{2}\right)$, qu'on écrit

$$
y_{2}=\sum_{l \in \delta(1) \backslash \delta(1)_{K}} \mu_{l} \rho_{l}
$$

avec les $\mu_{l}$ dans $\mathbf{R}_{>0}$.

Comme $z \in N$, on a $\left[N: N^{\prime}\right] z \in N^{\prime}$, donc $\left[N: N^{\prime}\right] z_{2} \in N^{\prime}$ Comme $y_{2}-z_{2} \in N^{\prime},\left[N: N^{\prime}\right] y_{2}$ est dans $N^{\prime}$. Ainsi les $\mu_{l}$ sont dans $\frac{1}{\left[N: N^{\prime}\right]} \mathbf{Z}_{>0}$.

Par définition de $\delta(1)_{K}$, pour tout $l$ de $\delta(1) \backslash \delta(1)_{K}$, il existe $i$ dans $K$ vérifiant $\left\langle\rho_{l}, \lambda_{i}^{\vee}\right\rangle \geqslant 1$ (rappelons que pour tout $i$ on a $\left\langle\rho_{l}, \lambda_{i}^{\vee}\right\rangle \geqslant 0$ ). Comme $y_{2}$ vérifie

$$
\forall i \in K, \quad\left\langle y_{2}, \lambda_{i}^{\vee}\right\rangle<\left\langle z, \lambda_{i}^{\vee}\right\rangle
$$

on a

$$
\mu_{l}<\operatorname{Sup}_{i \in K}\left\langle z, \lambda_{i}^{\vee}\right\rangle \leqslant\left\langle z, \lambda^{\vee}\right\rangle .
$$

Ainsi $\mathcal{C}\left(\delta(1) \backslash \delta(1)_{K}\right)\left(K, z_{2}\right)$ est fini et son cardinal est majoré par

$$
\left[N: N^{\prime}\right]^{\left[\delta(1) \backslash \delta(1)_{K}\right]}\left\langle z, \lambda^{\vee}\right\rangle^{\left[\delta(1) \backslash \delta(1)_{K}\right]},
$$


cette quantité étant elle-même majorée par

$$
\left(\left[N: N^{\prime}\right]\left\langle z, \lambda^{\vee}\right\rangle\right)^{\operatorname{rg}(N)} .
$$

Par ailleurs un élément $y_{2}$ de $\mathcal{C}\left(\delta(1) \backslash \delta(1)_{K}\right)\left(K, z_{2}\right)$ vérifie

$$
0 \leqslant\left\langle y_{2}, \lambda^{\vee}\right\rangle \leqslant \sum_{l \in \delta(1) \backslash \delta(1)_{K}}\left\langle z, \lambda^{\vee}\right\rangle\left\langle\rho_{l}, \lambda^{\vee}\right\rangle \leqslant \mathcal{M}\left\langle z, \lambda^{\vee}\right\rangle
$$

où on rappelle que

$$
\mathcal{M}=[I] \operatorname{Sup}_{l \in \Delta(1)}\left(\left\langle\rho_{l}, \lambda^{\vee}\right\rangle\right)
$$

En outre on a

$$
0 \geqslant\left\langle z_{1}-z_{1}^{\prime}, \lambda^{\vee}\right\rangle \geqslant-\sum_{l \in \delta(1)_{K}}\left\langle\rho_{l}, \lambda^{\vee}\right\rangle \geqslant-\mathcal{M}
$$

et finalement si $y$ est un élément de $F(\delta, K, z)$ on a

$$
-\mathcal{M} \leqslant\left\langle y, \lambda^{\vee}\right\rangle \leqslant \mathcal{M}\left\langle z, \lambda^{\vee}\right\rangle \text {. }
$$

Supposons $(N /\langle\Upsilon\rangle)^{\vee} \cap \Lambda^{\vee}=\{0\}$. Ceci équivaut à dire que si $\lambda \in \Lambda^{\vee}$ vérifie $\langle m, \lambda\rangle=0$ pour tout $m \in\langle\Upsilon\rangle$ alors $\lambda=0$. Mais si $\delta$ est de dimension maximale, les $\left(\rho_{l}\right)_{l \in \delta(1)}$ engendrent $\langle\Upsilon\rangle$, et donc si $K$ n'est pas vide, on ne peut avoir $\delta(1)_{K}=\delta(1)$.

Nous pouvons généraliser le lemme 7.5 de la manière suivante. On conserve les notations introduites avant l'énoncé du lemme 7.5. On considère en outre $\widetilde{I} \subset I$ un sous-ensemble strict de $I$ et $\widetilde{\Lambda} \subset \Lambda$ le cône engendré par les $\left(\lambda_{i}\right)_{i \in \widetilde{I}}$.

Soit

$$
\widetilde{\Upsilon_{z}}=\Upsilon \cap\left\{z+\widetilde{\Lambda} \cap N^{\prime}\right\}
$$

On veut étudier la série

$$
\mathcal{L}_{N, \widetilde{\Upsilon_{z}}}(T)=\sum_{\substack{y \in \Upsilon \cap N \\ y \in z+\widetilde{\Lambda} \cap N^{\prime}}} T^{y} .
$$

Nous écrivons d'abord

$$
\mathcal{L}_{N, \widetilde{\Upsilon_{z}}}(T)=\sum_{\delta \in \Delta} \mathcal{L}_{N, \widetilde{\Upsilon_{z}} \cap \operatorname{intrel}(\delta)}(T) .
$$


Pour $\delta \in \Delta$, notons qu'on peut écrire

$$
\begin{aligned}
& \mathcal{L}_{N, \widetilde{\Upsilon_{z}} \cap \operatorname{intrel}(\delta)}(s)=\sum_{y \in \operatorname{intrel}(\delta)} T^{y}=\sum_{y \in \operatorname{intrel}(\delta)} T^{y} . \\
& y-z \in \widetilde{\Lambda} \quad \forall i \in \widetilde{I},\left\langle\lambda_{i}^{\vee}, y\right\rangle \geqslant\left\langle\lambda_{i}^{\vee}, z\right\rangle \\
& y-z \in N^{\prime} \quad \forall i \in I \backslash \widetilde{I},\left\langle\lambda_{i}^{\vee}, y\right\rangle=\left\langle\lambda_{i}^{\vee}, z\right\rangle \\
& y-z \in N^{\prime}
\end{aligned}
$$

Pour tout sous-ensemble $K$ de $\widetilde{I}$ et tout cône $C$ de $N_{\mathbf{R}}$, soit $\widetilde{C}(K, z)$ le sousensemble de intrel $(C)$ formé des éléments $y$ vérifiant les conditions

$$
\forall i \in K,\left\langle\lambda_{i}^{\vee}, y\right\rangle<\left\langle\lambda_{i}^{\vee}, z\right\rangle
$$

et

$$
\forall i \in I \backslash \widetilde{I},\left\langle\lambda_{i}^{\vee}, y\right\rangle=\left\langle\lambda_{i}^{\vee}, z\right\rangle .
$$

On a alors une décomposition

$$
\mathcal{L}_{N, \widetilde{\Upsilon}_{z} \cap \operatorname{intrel}(\delta)}(T)=\sum_{K \subset \widetilde{I}}(-1)^{[K]} \mathcal{L}_{N, \widetilde{\delta}(K, z) z}(T) .
$$

Une légère adaptation de la preuve du lemme 7.5 permet alors de montrer le lemme suivant.

\section{Lemme 7.6}

Soient $\delta$ un cône de $\Delta$, et $K$ une partie de $\widetilde{I}$.

Soit $\widetilde{\delta(1)_{K}}$ le sous-ensemble de $\delta(1)$ défini par

$$
\widetilde{\delta(1)_{K}}=\left\{l \in \delta(1), \forall i \in K \cup(I \backslash \widetilde{I}), \quad\left\langle\rho_{l}, \lambda_{i}^{\vee}\right\rangle=0\right\} .
$$

On complète $\left(\rho_{l}\right)_{l \in \delta(1)}$ en une base $\left(\rho_{l}\right)_{l \in L}$ de $N^{\prime}$. Soit $N_{1}^{\prime}$ (respectivement $N_{2}^{\prime}$, respectivement $N_{3}^{\prime}$ ) le sous-module de $N$ engendré par les $\left(\rho_{l}\right)_{l \in \widetilde{\delta(1)_{K}}}$ (respectivement $\left(\rho_{l}\right)_{l \in \delta(1) \backslash \widetilde{\delta(1)_{K}}}$, respectivement $\left.\left(\rho_{l}\right)_{l \in L \backslash \delta(1)}\right)$.

On écrit $z=z_{1}+z_{2}+z_{3}$ avec pour $i=1,2,3, z_{i} \in\left(N_{i}^{\prime}\right)_{\boldsymbol{Q}}$.

On note $z_{1}^{\prime}$ l'unique élément de $N^{\prime}$ vérifiant

$$
z_{1}^{\prime}-z_{1}=\sum_{l \in \widetilde{\delta(1)_{K}}} \mu_{l} \rho_{l} \text { avec } 0 \leqslant \mu_{l}<1 .
$$

Soit $\widetilde{F}(\delta, K, z)$ l'ensemble des éléments qui s'écrivent $y_{2}+z_{1}-z_{1}^{\prime}$ où $y_{2}$ est un élément de $\mathrm{C}\left(\delta(1) \backslash \widetilde{\delta(1)_{K}}\right)(K, z)_{z_{2}}$.

Alors les ensembles $\widehat{\delta(1)_{K}}, \widetilde{\delta}(K, z)_{z}$ et $\widetilde{F}(\delta, K, z)$ vérifient les propriétés suivantes : 
1. Si $\widetilde{\delta}(K, z)_{z}$ est non vide, alors $z_{3}$ est un élément de $N^{\prime}$.

2. Si $z_{3}$ est un élément de $N^{\prime}$, on a une décomposition

$$
\mathcal{L}_{N, \widetilde{\delta}(K, z)_{z}}(T)=\mathcal{L}_{N^{\prime}, \operatorname{intrel}\left(\mathrm{e}\left(\widetilde{\delta(1)_{K}}\right)\right)}(T) \mathcal{L}_{N, \widetilde{F}(\delta, K, z)}(T)
$$

3. $\widetilde{F}(\delta, K, z)$ est fini. Plus précisément, on a la majoration

$$
[\widetilde{F}(\delta, K, z)] \leqslant\left(\left[N: N^{\prime}\right]\left\langle z, \lambda^{\vee}\right\rangle\right)^{\mathrm{rg}(N)} .
$$

4. Pour tout $y \in F \widetilde{(\delta, K, z)}$ on a

$$
-\mathcal{M} \leqslant\left\langle y, \lambda^{\vee}\right\rangle \leqslant \mathcal{M}\left\langle z, \lambda^{\vee}\right\rangle .
$$

5. On suppose en outre que $(N /\langle\Upsilon\rangle)^{\vee} \cap \Lambda^{\vee}=\{0\}$ et que $\delta$ est de dimension maximale. Alors $\widetilde{\delta(1)_{K}}$ est un sous-ensemble strict de $\delta(1)$.

\subsection{Comportement des fonctions étudiées par intégra- tion}

Rappelons que nous avons fixé un Z-module libre de rang fini $N$, et $\Lambda$ un cône simplicial de $N_{\mathbf{R}}$. On considère en outre désormais un sous groupe $M$ de $N$ tel que le quotient $\Gamma=N / M$ soit sans torsion. On notera $j$ l'application quotient $N \rightarrow \Gamma$ et $i$ le morphisme d'inclusion $M \rightarrow N$. On a donc une suite exacte de $\mathbf{Z}$-module libres de rang fini

$$
0 \longrightarrow M \stackrel{i}{\longrightarrow} N \stackrel{j}{\longrightarrow} \Gamma \longrightarrow 0
$$

et la suite exacte duale

$$
0 \longrightarrow \Gamma^{\vee} \stackrel{j^{\vee}}{\longrightarrow} N^{\vee} \stackrel{i^{\vee}}{\longrightarrow} M^{\vee} \longrightarrow 0 .
$$

Nous aurons en particulier par la suite à considérer la situation où $\Gamma^{\vee} \cap$ $\Lambda^{\vee}=\{0\}$. Cette hypothèse équivaut au fait que $i^{\vee}\left(\Lambda^{\vee}\right)$ est strictement convexe, ou encore que $\Lambda \cap M_{\mathbf{R}}=\left[i^{\vee}\left(\Lambda^{\vee}\right)\right]^{\vee}$ est d'intérieur non vide, ou encore que l'intérieur de $\Lambda$ rencontre $M$.

\section{Lemme 7.7}

Pour tout $s \in \mathcal{T}\left(\Lambda_{>0}^{\vee}\right)$ on a

$$
\mathcal{L}_{N, \Lambda \cap M_{\boldsymbol{R}}}\left(q^{-s}\right)=\mathcal{L}_{M, \Lambda \cap M_{\boldsymbol{R}}}\left(q^{-i^{\vee}(\boldsymbol{s})}\right) .
$$


Démonstration : En effet on peut écrire

$$
\begin{aligned}
\mathcal{L}_{N, \Lambda \cap M_{\mathbf{R}}}\left(q^{-s}\right) & =\sum_{y \in \Lambda \cap M_{\mathbf{R}} \cap N} q^{-\langle y, s\rangle} \\
& =\sum_{y \in \Lambda \cap M} q^{-\langle y, \boldsymbol{s}\rangle} \\
& =\sum_{y \in \Lambda \cap M} q^{-\langle i(y), \boldsymbol{s}\rangle} \\
& =\sum_{y \in \Lambda \cap M} q^{-\left\langle y, i^{\vee}(\boldsymbol{s})\right\rangle} \\
& =\mathcal{L}_{M, \Lambda \cap M_{\mathbf{R}}}\left(q^{-i^{\vee}(\boldsymbol{s})}\right) .
\end{aligned}
$$

\subsubsection{Le lemme technique : forme dépouillée}

\section{Lemme 7.8}

On a

$$
\int_{\Gamma_{\boldsymbol{U}}^{\vee}} \mathcal{L}_{N, \Lambda}\left(j_{\boldsymbol{U}}^{\vee}(\boldsymbol{z}) q^{-\boldsymbol{s}}\right) d \boldsymbol{z}=\mathcal{L}_{N, \Lambda \cap M_{\boldsymbol{R}}}\left(q^{-\boldsymbol{s}}\right) .
$$

Démonstration : Il suffit d'écrire

$$
\begin{aligned}
\int_{\Gamma_{\boldsymbol{U}}^{\vee}} \sum_{y \in \Lambda \cap N}\left\langle j_{\boldsymbol{U}}^{\vee}(\boldsymbol{z}), y\right\rangle q^{-\langle y, \boldsymbol{s}\rangle} d \boldsymbol{z} & =\sum_{y \in \Lambda \cap N} q^{-\langle y, \boldsymbol{s}\rangle} \int_{\Gamma_{\boldsymbol{U}}^{\vee}}\left\langle j_{\boldsymbol{U}}^{\vee}(\boldsymbol{z}), y\right\rangle d \boldsymbol{z} \\
& =\sum_{y \in \Lambda \cap N} q^{-\langle y, \boldsymbol{s}\rangle} \int_{\Gamma_{\boldsymbol{U}}^{\vee}}\langle\boldsymbol{z}, j(y)\rangle d \boldsymbol{z} \\
& =\sum_{\substack{y \in \Lambda \cap N \\
j(y)=0}} q^{-\langle y, \boldsymbol{s}\rangle} \\
& =\sum_{y \in \Lambda \cap M} q^{-\langle y, \boldsymbol{s}\rangle} \\
& =\mathcal{L}_{N, \Lambda \cap M_{\mathbf{R}}}\left(q^{-\boldsymbol{s}}\right)
\end{aligned}
$$

d'où le lemme.

\subsubsection{Le lemme technique : forme simple}

On se donne $a: \Lambda \cap N \rightarrow \mathrm{C}$ et $\varepsilon>0$ tels que la série définissant $\mathcal{L}_{N, \Lambda, a}\left(q^{-s}\right)$ converge absolument pour tout $s \in \mathcal{T}\left(\Lambda_{>-\varepsilon}^{\vee}\right)$. Comme déjà in- 
diqué, ceci équivaut à la condition suivante :

pour tout $\eta<\varepsilon$, la série $\sum_{y \in \Lambda \cap N}\left|a_{y}\right| q^{\eta\langle y, \lambda\rangle}$ est convergente.

Pour $\boldsymbol{s} \in \mathcal{T}\left(\Lambda_{>0}^{\vee}\right)$, posons

$$
f_{1}(\boldsymbol{s})=\int_{\Gamma_{U}^{\vee}} \mathcal{L}_{N, \Lambda, a}\left(j_{\boldsymbol{U}}^{\vee}(\boldsymbol{z}) q^{-\boldsymbol{s}}\right) \mathcal{L}_{N, \Lambda}\left(j_{\boldsymbol{U}}^{\vee}(\boldsymbol{z}) q^{-\boldsymbol{s}}\right) d \boldsymbol{z} .
$$

Cela définit une fonction $f_{1}$ holomorphe sur $\mathcal{T}\left(\Lambda_{>0}^{\vee}\right)$.

Remarque 7.9 : D'après le lemme 5.51, on peut, dans la définition (7.16) de $f_{1}$, remplacer l'intégrale sur $\Gamma_{\boldsymbol{U}}^{\vee}$ par une intégrale sur $\Gamma_{\boldsymbol{U}}^{\prime}$ où $\Gamma^{\prime}$ est n'importe quel sous-groupe d'indice fini de $\Gamma^{\vee}$.

\section{Lemme 7.10}

On fait l'hypothèse que $\Gamma^{\vee} \cap \Lambda^{\vee}=\{0\}$. Alors la fonction

$$
\boldsymbol{s} \mapsto f_{1}(s)-\mathcal{L}_{N, \Lambda, a}(1) \mathcal{L}_{N, \Lambda \cap M_{\mathbf{R}}}\left(q^{-s}\right)
$$

est admissible de multiplicité supérieure à $1-\operatorname{rg}(M)$.

Démonstration : On a pour tout $s$ de $\mathcal{T}\left(\Lambda_{>0}^{\vee}\right)$,

$$
\begin{aligned}
\mathcal{L}_{N, \Lambda, a}\left(j_{\boldsymbol{U}}^{\vee}(\boldsymbol{z}) q^{-\boldsymbol{s}}\right) \mathcal{L}_{N, \Lambda}\left(j_{\boldsymbol{U}}^{\vee}(\boldsymbol{z}) q^{-\boldsymbol{s}}\right) & \left(\sum_{y \in \Lambda \cap N} a_{y}\left\langle j_{\boldsymbol{U}}^{\vee}(\boldsymbol{z}), y\right\rangle q^{-\langle y, \boldsymbol{s}\rangle}\right)\left(\sum_{y \in \Lambda \cap N}\left\langle j_{\boldsymbol{U}}^{\vee}(\boldsymbol{z}), y\right\rangle q^{-\langle y, \boldsymbol{s}\rangle}\right) \\
= & \sum_{\left(y_{0}, y_{1}\right) \in(\Lambda \cap N)^{2}} a_{y_{1}}\left\langle j_{\boldsymbol{U}}^{\vee}(\boldsymbol{z}), y_{0}+y_{1}\right\rangle q^{-\left\langle y_{0}+y_{1}, \boldsymbol{s}\right\rangle} .
\end{aligned}
$$

d'où

$\int_{\Gamma_{\boldsymbol{U}}^{\vee}} \mathcal{L}_{N, \Lambda, a}\left(j_{\boldsymbol{U}}^{\vee}(\boldsymbol{z}) q^{-\boldsymbol{s}}\right) \mathcal{L}_{N, \Lambda}\left(j_{\boldsymbol{U}}^{\vee}(\boldsymbol{z}) q^{-\boldsymbol{s}}\right) d \boldsymbol{z}=\sum_{\left(y_{0}, y_{1}\right) \in(\Lambda \cap N)^{2}} a_{y_{1}} q^{-\left\langle y_{0}+y_{1}, \boldsymbol{s}\right\rangle} \int_{\Gamma_{\boldsymbol{U}}^{\vee}}\left\langle\boldsymbol{z}, j\left(y_{0}+y_{1}\right)\right\rangle d \boldsymbol{z}$

$$
\begin{aligned}
& =\sum_{y_{1} \in \Lambda \cap N} a_{y_{1}} \sum_{\substack{y_{0} \in \Lambda \cap N \\
j\left(y_{0}+y_{1}\right)=0}} q^{-\left\langle y_{0}+y_{1}, s\right\rangle} \\
& =\sum_{y_{1} \in \Lambda \cap N} a_{y_{1}} \sum_{\substack{y \in \Lambda \cap M \\
y \in y_{1}+\Lambda \cap N}} q^{-\langle y, s\rangle} .
\end{aligned}
$$


On applique alors le lemme 7.4 avec $\Upsilon=\Lambda \cap M_{\mathbf{R}}$. On obtient la décomposition

$$
f_{1}(s)=\sum_{\delta \in \Delta} \sum_{K \subset I}(-1)^{[K]} \mathcal{L}_{N, \operatorname{intrel}\left(\mathcal{e}\left(\delta(1)_{K}\right)\right)}\left(q^{-s}\right) \sum_{y_{1} \in \Lambda \cap N} a_{y_{1}} \mathcal{L}_{N, F\left(\delta, K, y_{1}\right)}\left(q^{-s}\right) .
$$

Soient $\delta$ et $K$ donnés. D'après les majorations (7.11) et (7.12) on a pour tout $\eta$

$$
\sum_{y_{1} \in \Lambda \cap N}\left|a_{y_{1}}\right| \sum_{y \in F\left(\delta, K, y_{1}\right)} q^{\eta\langle y, \lambda\rangle} \leqslant \sum_{y_{1} \in \Lambda \cap N}\left|a_{y_{1}}\right|\left\langle y_{1}, \lambda\right\rangle^{\operatorname{rg}(N)} q^{\eta M\left\langle y_{1}, \lambda\right\rangle} .
$$

et cette dernière série converge pour tout $\eta<\frac{\varepsilon}{M}$. Ainsi la série

$$
\sum_{y \in \Lambda \cap N}\left(\sum_{\substack{y_{1} \in \Lambda \cap N \\ y \in F\left(\delta, K, y_{1}\right)}} a_{y_{1}}\right) q^{-\langle y, s\rangle}
$$

définit une fonction admissible de multiplicité supérieure à 0 . Comme $\Gamma^{\vee} \cap$ $\Lambda^{\vee}=\{0\}$, d'après le point $7.4 \mathrm{du}$ lemme 7.4 la fonction

$$
\mathcal{L}_{N, \operatorname{intrel}\left(\mathcal{e}\left(\delta(1)_{K}\right)\right)}\left(q^{-\boldsymbol{s}}\right) \sum_{y_{1} \in \Lambda \cap N} a_{y_{1}} \mathcal{L}_{N, F\left(\delta, K, y_{1}\right)}\left(q^{-\boldsymbol{s}}\right) .
$$

est, si $K \neq \varnothing$, une fonction admissible de multiplicité supérieure à $1-\operatorname{rg}(M)$.

La contribution des termes correspondant à $K=\varnothing$ dans la décomposition ci-dessus est

$$
\left(\sum_{y_{1} \in \Lambda \cap N} a_{y_{1}}\right) \times\left(\sum_{\delta \in \Delta} \mathcal{L}_{N, \operatorname{intrel}(\delta)}\left(q^{-s}\right)\right)=\mathcal{L}_{N, \Lambda, a}(1) \times \mathcal{L}_{M, \Lambda \cap M_{\mathbf{R}}}\left(q^{-s}\right)
$$

d'où le lemme.

\subsubsection{Le lemme technique : forme générale}

Comme dans la sous-section précédente, on commence par se donner $a$ : $\Lambda \cap N \rightarrow \mathrm{C}$ et $\varepsilon>0$ tels que la série définissant $\mathcal{L}_{N, \Lambda, a}\left(q^{-s}\right)$ converge absolument pour tout $s \in \mathcal{T}\left(\Lambda_{>-\varepsilon}^{\vee}\right)$.

On se donne en outre $N^{\prime}$ un sous-groupe d'indice fini de $N$.

Pour $s \in \mathcal{T}\left(\Lambda_{>0}^{\vee}\right)$, posons

$$
f_{2}(\boldsymbol{s})=\int_{\boldsymbol{z} \in \Gamma_{\boldsymbol{U}}^{\vee}} \mathcal{L}_{N, \Lambda, a}\left(j_{\boldsymbol{U}}^{\vee}(\boldsymbol{z}) q^{-\boldsymbol{s}}\right) \mathcal{L}_{N^{\prime}, \Lambda}\left(j_{\boldsymbol{U}}^{\vee}(\boldsymbol{z}) q^{-\boldsymbol{s}}\right) d \boldsymbol{z} .
$$


Cela définit une fonction $f_{2}$ holomorphe sur $\mathcal{T}\left(\Lambda_{>0}^{\vee}\right)$.

Remarque 7.11: D'après le lemme 5.51, on peut, dans la définition (7.27) de $f_{2}$, remplacer l'intégrale sur $\Gamma_{\boldsymbol{U}}^{\vee}$ par une intégrale sur $\Gamma_{\boldsymbol{U}}^{\prime}$ où $\Gamma^{\prime}$ est n'importe quel sous-groupe d'indice fini de $\Gamma^{\vee}$.

\section{Lemme 7.12}

On suppose que $\Gamma^{\vee} \cap \Lambda^{\vee}=\{0\}$.

1. Il existe alors une fonction $g$ admissible de multiplicité supérieure à zéro telle que la fonction

$$
\boldsymbol{s} \mapsto f_{2}(\boldsymbol{s})-g(\boldsymbol{s}) \mathcal{L}_{N^{\prime}, \Lambda \cap M_{\boldsymbol{R}}}\left(q^{-\boldsymbol{s}}\right)
$$

est admissible de multiplicité supérieure à $1-\operatorname{rg}(M)$.

2. On suppose en outre que la condition suivante est vérifiée : pour tout $y \in \Lambda \cap N$, si $a_{y}$ est non nul alors $j(y)$ est un élément de $j\left(N^{\prime}\right)$. Alors on peut choisir $g$ de sorte qu'on ait

$$
g(0)=\mathcal{L}_{N, \Lambda, a}(1) .
$$

Démonstration : On a pour tout $\boldsymbol{s}$ de $\mathcal{T}\left(\Lambda_{>0}^{\vee}\right)$, et tout $\boldsymbol{z}$ de $\Gamma_{\boldsymbol{U}}^{\vee}$

$$
\begin{aligned}
\mathcal{L}_{N, \Lambda, a} & \left(j_{\boldsymbol{U}}^{\vee}(\boldsymbol{z}) q^{-\boldsymbol{s}}\right) \mathcal{L}_{N^{\prime}, \Lambda}\left(j_{\boldsymbol{U}}^{\vee}(\boldsymbol{z}) q^{-\boldsymbol{s}}\right) \\
= & \left(\sum_{y \in \Lambda \cap N} a_{y}\langle\boldsymbol{z}, j(y)\rangle q^{-\langle y, \boldsymbol{s}\rangle}\right)\left(\sum_{y \in \Lambda \cap N^{\prime}}\langle\boldsymbol{z}, j(y)\rangle q^{-\langle y, \boldsymbol{s}\rangle}\right)
\end{aligned}
$$

d'où

$$
\begin{aligned}
& \int_{\boldsymbol{z} \in \Gamma_{U}^{\vee}} \mathcal{L}_{N, \Lambda, a}\left(j_{\boldsymbol{U}}^{\vee}(\boldsymbol{z}) q^{-\boldsymbol{s}}\right) \mathcal{L}_{N^{\prime}, \Lambda}\left(j_{\boldsymbol{U}}^{\vee}(\boldsymbol{z}) q^{-\boldsymbol{s}}\right) d \boldsymbol{z} \\
= & \sum_{(y, z) \in\left(\Lambda \cap N^{\prime}\right) \times(\Lambda \cap N)} a_{z} q^{-\langle y+z, \boldsymbol{s}\rangle} \int_{\Gamma_{\boldsymbol{U}}^{\vee}}\langle\boldsymbol{z}, j(y+z)\rangle d \boldsymbol{z} \\
= & \sum_{z \in \Lambda \cap N} a_{z} \sum_{\substack{y \in \Lambda \cap N^{\prime} \\
j(y+z)=0}} q^{-\langle y+z, \boldsymbol{s}\rangle} \\
= & \sum_{z \in \Lambda \cap N} a_{z} \sum_{\substack{y \in \Lambda \cap N^{\prime} \\
y+z \in M}} q^{-\langle y+z, \boldsymbol{s}\rangle} \\
= & \sum_{z \in \Lambda \cap N} a_{z} \sum_{\substack{y \in \Lambda \cap M \\
y \in z+\Lambda \cap N^{\prime}}} q^{-\langle y, s\rangle} .
\end{aligned}
$$


On applique alors le lemme 7.5 avec $\Upsilon=\Lambda \cap M_{\mathbf{R}}$. On reprend à cet effet les notations du lemme 7.5. On obtient la décomposition

$$
f_{2}(\boldsymbol{s})=\sum_{\delta \in \Delta} \sum_{K \subset I}(-1)^{[K]} \mathcal{L}_{N^{\prime}, \operatorname{intrel}\left(\mathcal{e}\left(\delta(1)_{K}\right)\right)}\left(q^{-\boldsymbol{s}}\right) \sum_{\substack{z \in \Lambda \cap N \\ z 3 \in N^{\prime}}} a_{z} \mathcal{L}_{N, F(\delta, K, z)}\left(q^{-\boldsymbol{s}}\right) .
$$

Soient $\delta$ et $K$ donnés. D'après les majorations (7.38) et (7.39) on a pour tout $\eta$ la majoration

$$
\sum_{\substack{z \in \Lambda \cap N \\ z_{3} \in N^{\prime}}}\left|a_{z}\right| \sum_{y \in F(\delta, K, z)} q^{\eta\langle y, \lambda\rangle} \leqslant \sum_{z \in \Lambda \cap N}\left|a_{z}\right|\left(\left[N: N^{\prime}\right]\langle z, \lambda\rangle\right)^{\operatorname{rg}(N)} q^{\eta \mathcal{M}\langle z, \lambda\rangle} .
$$

D'après la condition (7.15), cette dernière série converge pour tout $\eta<\frac{\varepsilon}{\mathcal{M}}$. Ainsi la série

$$
\sum_{\substack{z \in \Lambda \cap N \\ z 3 \in N^{\prime}}} a_{z} \mathcal{L}_{N, F(\delta, K, z)}\left(q^{-s}\right)
$$

définit une fonction admissible de multiplicité supérieure à 0 .

Comme $\Gamma^{\vee} \cap \Lambda^{\vee}=\{0\}$, l'assertion 6 du lemme 7.5 montre que si $K \neq \varnothing$ ou $\operatorname{dim}(\delta)<\operatorname{rg}(M)$, la fonction

$$
\mathcal{L}_{N^{\prime}, \operatorname{intrel}\left(\mathcal{C}\left(\delta(1)_{K}\right)\right)}\left(q^{-\boldsymbol{s}}\right) \sum_{z \in \Lambda \cap N} a_{z} L_{N, F(\delta, K, z)}\left(q^{-\boldsymbol{s}}\right) .
$$

est une fonction admissible de multiplicité supérieure à $1-\operatorname{rg}(M)$.

Écrivons la contribution des termes correspondant à $K=\varnothing$ et $\operatorname{dim}(\delta)=$ $\operatorname{rg}(M)$ dans la décomposition (7.36). Pour cela, on commence par remarquer la chose suivante : soit $\delta$ tel que $\operatorname{dim}(\delta)=\operatorname{rg}(M)$ et $K=\varnothing$. Avec les notations du lemme 7.5, on a $N_{1}^{\prime}=M \cap N^{\prime}, N_{2}^{\prime}=0$ et $N^{\prime}=\left(N^{\prime} \cap M\right) \oplus N_{3}^{\prime}$. Ainsi, si $z \in N$ s'écrit $z=z_{1}+z_{3}$ avec $z_{1} \in\left(N^{\prime} \cap M\right)_{\mathbf{Q}}$ et $z_{3} \in\left(N_{3}^{\prime}\right)_{\mathbf{Q}}$, la condition $z_{3} \in N^{\prime}$ est équivalente à la condition $j(z) \in j\left(N^{\prime}\right)$. Ainsi la contribution considérée s'écrit

$$
\left(\sum_{\substack{z \in \Lambda \cap N, j(z) \in j\left(N^{\prime}\right)}} a_{z} q^{-\left\langle z_{1}-z_{1}^{\prime}, s\right\rangle}\right) \times\left(\sum_{\substack{\delta \in \Delta \\ \operatorname{dim}(\delta)=\operatorname{rg}(M)}} \mathcal{L}_{N^{\prime}, \text { intrel }(\delta)}\left(q^{-s}\right) .\right)
$$

Pour tout $z \in \Lambda \cap N$, on a $-\mathcal{M} \leqslant\left\langle z_{1}-z_{1}^{\prime}, \lambda\right\rangle \leqslant 0$. Ainsi, pour $\eta \geqslant 0$ on a

$$
\sum_{\substack{z \in \Lambda \cap N, j(z) \in j\left(N^{\prime}\right)}}\left|a_{z} q^{\eta\left\langle z_{1}-z_{1}^{\prime}, \lambda\right\rangle}\right| \leqslant \sum_{z \in \Lambda \cap N,}\left|a_{z}\right|<+\infty
$$


et pour $\eta \leqslant 0$ on a

$$
\sum_{\substack{z \in \Lambda \cap N, j(z) \in j\left(N^{\prime}\right)}}\left|a_{z} q^{\eta\left\langle z_{1}-z_{1}^{\prime}, \lambda\right\rangle}\right| \leqslant q^{-\eta \mathcal{M}} \sum_{z \in \Lambda \cap N,}\left|a_{z}\right|<+\infty .
$$

Ceci montre que la fonction $g$ définie par

$$
g(\boldsymbol{s})=\sum_{\substack{z \in \Lambda \cap N, j(z) \in j\left(N^{\prime}\right)}} a_{z} q^{\left\langle z_{1}-z_{1}^{\prime}, s\right\rangle}
$$

est admissible de multiplicité positive.

Comme on a la décomposition

$$
\mathcal{L}_{N^{\prime}, \Lambda \cap M_{\mathbf{R}}}\left(q^{-s}\right)=\sum_{\delta \in \Delta} \sum_{K \subset I}(-1)^{[K]} \mathcal{L}_{N^{\prime}, \operatorname{intrel}\left(\mathfrak{e}\left(\delta(1)_{K}\right)\right)}\left(q^{-s}\right)
$$

et que, pour $K \neq \varnothing$ ou $\operatorname{dim}(\delta)<\operatorname{rg}(M)$, la fonction $\boldsymbol{s} \mapsto \mathcal{L}_{N^{\prime}, \operatorname{intrel}\left(\mathfrak{e}\left(\delta(1)_{K}\right)\right)}\left(q^{-\boldsymbol{s}}\right)$ est admissible de multiplicité supérieure à $1-\operatorname{rg}(M)$, on déduit de ce qui précède que

$$
\boldsymbol{s} \mapsto f_{2}(s)-g(\boldsymbol{s}) \mathcal{L}_{N^{\prime}, \Lambda \cap M_{\mathbf{R}}}\left(q^{-\boldsymbol{s}}\right)
$$

est admissible de multiplicité supérieure à $1-\operatorname{rg}(M)$.

Supposons à présent que pour $z \in \Lambda \cap N$ la condition $a_{z} \neq 0$ entraîne $j(z) \in j\left(N^{\prime}\right)$. On a alors l'égalité

$$
\sum_{\substack{z \in \Lambda \cap N, j(z) \in j\left(N^{\prime}\right)}} a_{z}=\sum_{z \in \Lambda \cap N} a_{z}
$$

ce qui signifie exactement que $g(0)=\mathcal{L}_{N, \Lambda, a}(1)$.

Remarque 7.13 : S'il existe $y$ tel que $a_{y} \neq 0$ et $j(y) \notin j\left(N^{\prime}\right)$, la conclusion du point $2 \mathrm{du}$ lemme 7.12 peut être mise en défaut : il se peut que $f_{2}(\boldsymbol{s})$ soit admissible de multiplicité supérieure à $1-\operatorname{rg}(M)$, même si $\mathcal{L}_{N, \Lambda, a}(1)$ est non nul. En d'autres termes, dans ce cas de figure, on «perd» le contrôle du terme principal (ce qu'il faudra bien sûr éviter absolument lors de l'application au calcul de la fonction zêta des hauteurs).

À titre d'exemple, considérons le cas où $N=\mathbf{Z}^{2}, \Lambda=\mathbf{R}_{\geqslant 0}^{2}, M=\mathbf{Z}(1,1)$, $N^{\prime}=2 N$, et $a: \mathbf{N}^{2} \rightarrow \mathbf{C}$ est donnée par $a_{(2,1)}=1$ et $a_{y}=0$ si $y \neq(2,1)$. On a alors $j(1,0)=-j(0,1)$ et on identifie $\Gamma$ à $\mathbf{Z}$ au moyen de la base $j(1,0)$. 
Pour $\boldsymbol{z} \in \Gamma_{\boldsymbol{U}}^{\vee} \stackrel{\sim}{\rightarrow} \boldsymbol{U}$, on a alors

$$
\begin{aligned}
\mathcal{L}_{N, \Lambda, a}\left(j_{\boldsymbol{U}}^{\vee}(\boldsymbol{z}) q^{-\boldsymbol{s}}\right) \mathcal{L}_{N^{\prime}, \Lambda}\left(j_{\boldsymbol{U}}^{\vee}(\boldsymbol{z}) q^{-\boldsymbol{s}}\right) & =\frac{\boldsymbol{z}^{j(2,1)} q^{-2 s_{1}-s_{2}}}{\left(1-\boldsymbol{z}^{j(2,0)} q^{-2 s_{1}}\right)\left(1-\boldsymbol{z}^{j(0,2)} q^{\left.-2 s_{2}\right)}\right.} \\
& =\frac{\boldsymbol{z} q^{-2 s_{1}-s_{2}}}{\left(1-\boldsymbol{z}^{2} q^{-2 s_{1}}\right)\left(1-\boldsymbol{z}^{-2} q^{-2 s_{2}}\right)} \\
& =\sum_{\left(n_{1}, n_{2}\right) \in \mathbf{N}^{2}} \boldsymbol{z}^{2 n_{1}-2 n_{2}+1} q^{-2\left(n_{1}+1\right) s_{1}-\left(2 n_{2}+1\right) s_{2}}
\end{aligned}
$$

et de cette dernière expression, on déduit aussitôt qu'on a

$$
\int_{\boldsymbol{z} \in \Gamma_{\boldsymbol{U}}^{\vee}} \mathcal{L}_{N, \Lambda, a}\left(j_{\boldsymbol{U}}^{\vee}(\boldsymbol{z}) q^{-\boldsymbol{s}}\right) \mathcal{L}_{N^{\prime}, \Lambda}\left(j_{\boldsymbol{U}}^{\vee}(\boldsymbol{z}) q^{-\boldsymbol{s}}\right)=0 .
$$

On pourra constater par contre que si on définit $a$ par $a_{(3,1)}=1$ et $a_{y}=0$ si $y \neq(3,1)$, les hypothèses du point 2 du lemme 7.12 sont vérifiées. De fait, un calcul similaire montre qu'on a alors

$$
\begin{aligned}
\int_{\boldsymbol{z} \in \Gamma_{\boldsymbol{U}}^{\vee}} \mathcal{L}_{N, \Lambda, a}\left(j_{\boldsymbol{U}}^{\vee}(\boldsymbol{z}) q^{-\boldsymbol{s}}\right) \mathcal{L}_{N^{\prime}, \Lambda}\left(j_{\boldsymbol{U}}^{\vee}(\boldsymbol{z}) q^{-\boldsymbol{s}}\right) & =\frac{q^{-3 s_{1}-3 s_{2}}}{1-q^{-2 s_{1}-2 s_{2}}} \\
& =q^{-3 s_{1}-3 s_{2}} \mathcal{L}_{N^{\prime}, \Lambda \cap M_{\mathbf{R}}}\left(q^{-\left(s_{1}, s_{2}\right)}\right) .
\end{aligned}
$$

Le lemme 7.12 va en fait nous servir à traiter le terme principal de la fonction zêta des hauteurs, qui correspond (à une constante multiplicative près) au terme $I_{1}$ dans la formule (5.87). Nous avons besoin d'un autre lemme pour traiter les termes restants, à savoir les $I_{\chi}$, pour $\chi \in \mathcal{U}_{T} \backslash \operatorname{Ker}\left(\gamma^{*}\right)$, apparasissant dans cette même formule. On conserve les notations introduites avant l'énoncé du lemme 7.12. On considère en outre $\widetilde{I} \subset I$ un sous-ensemble strict de $I$, et $\widetilde{\Lambda} \subset \Lambda$ le cône engendré par les $\left(\lambda_{i}\right)_{i \in \widetilde{I}}$.

Si on suppose que $\Gamma$ vérifie $\Gamma^{\vee} \cap \Lambda^{\vee}=\{0\}, \widetilde{\Lambda} \cap M_{\mathbf{R}}$ est d'intérieur vide dans $M_{\mathbf{R}}$. En effet ce cône est le dual du cône $i(\widetilde{\Lambda})$, qui contient la droite engendrée par l'élément non nul $i\left(\lambda_{i_{0}}\right)$, où $i_{0} \notin I$.

Pour $s \in \mathcal{T}\left(\Lambda_{>0}^{\vee}\right)$, posons

$$
f_{3}(\boldsymbol{s})=\int_{\Gamma_{\boldsymbol{U}}^{\vee}} \mathcal{L}_{N, \Lambda, a}\left(j_{\boldsymbol{U}}^{\vee}(\boldsymbol{z}) q^{-\boldsymbol{s}}\right) \mathcal{L}_{N^{\prime}, \widetilde{\Lambda}}\left(j_{\boldsymbol{U}}^{\vee}(\boldsymbol{z}) q^{-\boldsymbol{s}}\right) d \boldsymbol{z} .
$$


Cela définit une fonction $f_{3}$ holomorphe sur $\mathcal{T}\left(\Lambda_{>0}^{\vee}\right)$.

\section{Lemme 7.14}

On fait l'hypothèse que $\Gamma^{\vee} \cap \Lambda^{\vee}=\{0\}$. Alors la fonction $f_{3}$ est admissible de multiplicité supérieure à $1-\operatorname{rg}(M)$.

Démonstration : On a pour tout $\boldsymbol{s}$ de $\mathcal{T}\left(\Lambda_{>0}^{\vee}\right)$, et tout $\boldsymbol{z}$ de $\Gamma_{\boldsymbol{U}}^{\vee}$

$$
\begin{aligned}
\mathcal{L}_{N, \Lambda, a} & \left(j_{\boldsymbol{U}}^{\vee}(\boldsymbol{z}) q^{-\boldsymbol{s}}\right) \mathcal{L}_{N^{\prime}, \widetilde{\Lambda}}\left(j_{\boldsymbol{U}}^{\vee}(\boldsymbol{z}) q^{-\boldsymbol{s}}\right) \\
= & \left(\sum_{y \in \Lambda \cap N} a_{y}\langle\boldsymbol{z}, j(y)\rangle q^{-\langle y, \boldsymbol{s}\rangle}\right)\left(\sum_{y \in \widetilde{\Lambda} \cap N^{\prime}}\langle\boldsymbol{z}, j(y)\rangle q^{-\langle y, \boldsymbol{s}\rangle}\right)
\end{aligned}
$$

d'où

$$
\begin{aligned}
& \int_{\Gamma_{\boldsymbol{U}}^{\vee}} \mathcal{L}_{N, \Lambda, a}\left(j_{\boldsymbol{U}}^{\vee}(\boldsymbol{z}) q^{-\boldsymbol{s}}\right) \mathcal{L}_{N^{\prime}, \widetilde{\Lambda}}\left(j_{\boldsymbol{U}}^{\vee}(\boldsymbol{z}) q^{-\boldsymbol{s}}\right) d \boldsymbol{z} \\
= & \sum_{(y, z) \in\left(\widetilde{\Lambda} \cap N^{\prime}\right) \times(\Lambda \cap N)} a_{z} q^{-\langle y+z, \boldsymbol{s}\rangle} \int_{\Gamma_{\boldsymbol{U}}^{\vee}} \boldsymbol{z}^{j(y+z)} d \boldsymbol{z} \\
= & \sum_{z \in \Lambda \cap N} a_{z} \sum_{\substack{y \in \widetilde{\Lambda} \cap N^{\prime} \\
j(y+z)=0}} q^{-\langle y+z, \boldsymbol{s}\rangle} \\
= & \sum_{z \in \Lambda \cap N} a_{z} \sum_{\substack{y \in \widetilde{\Lambda} \cap N^{\prime} \\
y+z \in M}} q^{-\langle y+z, \boldsymbol{s}\rangle} \\
= & \sum_{z \in \Lambda \cap N} a_{z} \sum_{\substack{y \in \Lambda \cap M \\
y \in z+\widetilde{\Lambda} \cap N^{\prime}}} q^{-\langle y, s\rangle} .
\end{aligned}
$$

On applique alors le lemme 7.6 avec $\Upsilon=\Lambda \cap M_{\mathbf{R}}$. On reprend à cet effet les notations du lemme 7.6. On obtient la décomposition

$$
f_{3}(\boldsymbol{s})=\sum_{\delta \in \Delta} \sum_{K \subset \widetilde{I}}(-1)^{[K]} \mathcal{L}_{N^{\prime}, \operatorname{intrel}\left(\mathfrak{e}\left(\widetilde{\left.\delta(1)_{K}\right)}\right)\right.}\left(q^{-\boldsymbol{s}}\right) \sum_{\substack{z \in \Lambda \cap N \\ z_{3} \in N^{\prime}}} a_{z} \mathcal{L}_{N, \widetilde{F}(\delta, K, z)}\left(q^{-\boldsymbol{s}}\right)
$$

Soient $\delta$ et $K$ donnés. D'après les majorations (7.74) et (7.75) on a pour tout $\eta$ la majoration

$$
\sum_{\substack{z \in \Lambda \cap N \\ z \\ z \in N^{\prime}}}\left|a_{z}\right| \sum_{y \in \tilde{F}(\delta, K, z)} q^{\eta\langle y, \lambda\rangle} \leqslant \sum_{z \in \Lambda \cap N}\left|a_{z}\right|(d\langle z, \lambda\rangle)^{\operatorname{rg}(N)} q^{\eta \mathcal{M}\langle z, \lambda\rangle} .
$$


D'après la condition (7.15), cette dernière série converge pour tout $\eta<\frac{\varepsilon}{\mathcal{M}}$. Ainsi la série

$$
\sum_{\substack{z \in \Lambda \cap N \\ z \\ z \in N^{\prime}}} a_{z} L_{N, F(\delta, K, z)}\left(q^{-s}\right)
$$

définit une fonction admissible de multiplicité supérieure à 0 .

Comme $\Gamma^{\vee} \cap \Lambda^{\vee}=\{0\}$, le point 5 du lemme 7.6 montre que la fonction

$$
s \mapsto \mathcal{L}_{N^{\prime}, \operatorname{intrel}\left(\mathcal{e}\left(\widetilde{\left.\delta(1)_{K}\right)}\right)\right.}\left(q^{-s}\right) \sum_{z \in \Lambda \cap N} a_{z} \mathcal{L}_{N, \widetilde{F}(\delta, K, z)}\left(q^{-s}\right)
$$

est une fonction admissible de multiplicité supérieure à $1-\operatorname{rg}(M)$.

\section{Application aux fonctions zêta des hauteurs}

\subsection{Préliminaires}

Rappelons que $X(T)^{G}$ s'identifie à un sous-groupe de $\mathbf{Z}^{\Sigma(1) / G}$ via la suite exacte

$$
0 \longrightarrow X(T)^{G} \stackrel{\gamma}{\longrightarrow} \mathbf{Z}^{\Sigma(1) / G} \stackrel{\pi}{\longrightarrow} \operatorname{Pic}\left(X_{\Sigma}\right) \longrightarrow H^{1}(G, X(T)) \longrightarrow 0
$$

tirée de la suite exacte (4.11) en prenant les $G$-invariants.

Soit $m \in X(T)^{G}$ vérifiant

$$
\forall \alpha \in \Sigma(1) / G, \quad\left\langle m, \rho_{\alpha}\right\rangle \geqslant 0 .
$$

Alors on a

$$
\forall \alpha \in \Sigma(1), \quad \forall \rho \in \alpha, \quad\langle m, \rho\rangle \geqslant 0 .
$$

L'éventail $\Sigma$ étant complet, ses rayons engendrent $X(T)^{\vee}$, ce qui entraîne $m=0$. Nous avons donc

$$
X(T)^{G} \cap \mathbf{Z}_{\geqslant 0}^{\Sigma(1) / G}=\{0\}
$$

(on aurait $\mathrm{pu}$ aussi invoquer directement le fait général que $C_{\mathrm{eff}}\left(X_{\Sigma}\right)$ est strictement convexe).

Dans le cas fonctionnel, on tire par ailleurs de (4.11) un complexe

$$
\mathcal{D}_{T_{\mathrm{NS}}} \longrightarrow \mathcal{D}_{T_{P_{\Sigma}}} \longrightarrow \mathcal{D}_{T}
$$

Notons $\mathcal{D}_{T_{\mathrm{NS}}}^{0}$ le noyau du morphisme $\mathcal{D}_{T_{P_{\Sigma}}} \rightarrow \mathcal{D}_{T}$. Rappelons que nous avons noté $\mathcal{D}_{T}^{0}$ l'image de ce morphisme dans $\mathcal{D}_{T}$. On a donc une suite exacte de Z-modules libre de rang fini.

$$
0 \longrightarrow \mathcal{D}_{T_{\mathrm{NS}}}^{0} \stackrel{\pi^{\vee}}{\longrightarrow} \mathcal{D}_{T_{P_{\Sigma}}} \stackrel{\gamma^{\vee}}{\longrightarrow} \mathcal{D}_{T}^{0} \longrightarrow 0
$$


et la suite exacte duale

$$
0 \longrightarrow\left(\mathcal{D}_{T}^{0}\right)^{\vee} \stackrel{\gamma}{\longrightarrow}\left(\mathcal{D}_{T_{P_{\Sigma}}}\right)^{\vee} \stackrel{\pi}{\longrightarrow}\left(\mathcal{D}_{T_{\mathrm{NS}}}^{0}\right)^{\vee} \longrightarrow 0 .
$$

Comme (4.11) est une résolution flasque de $X(T)$ (lemme 4.2), d'après la proposition 3.40 , l'indice dans $\mathcal{D}_{T_{\mathrm{NS}}}^{0}$ de l'image de $\mathcal{D}_{T_{\mathrm{NS}}}$ dans $\mathcal{D}_{T_{P_{\Sigma}}}$ est égal à

$$
\frac{\left[H^{1}(G, X(T))\right]\left[\mathcal{C}_{T_{\mathrm{NS}}}\right]\left[\mathcal{C}_{T}\right] \mathcal{K}_{T}}{\left[\mathcal{C}_{T_{P_{\Sigma}}}\right]} .
$$

On pose

$$
C_{0}=\lim _{s \rightarrow 1}(s-1)^{[\Sigma(1) / G]} \int_{\overline{T(K)} \cap T\left(\mathbf{A}_{K}\right)} H\left(-s \varphi_{0}, t\right) \omega_{T}(t) .
$$

On montrera à la sous-section 8.4.1 que $C_{0}$ est non nulle.

\subsection{Application du lemme technique dans le cas ari- thmétique}

Rappelons que d'après le corollaire 5.48 , on a pour tout élément $s$ de $\mathcal{T}\left(\mathbf{R}_{>0}^{\Sigma(1) / G}\right)$ la représentation intégrale

$$
\begin{aligned}
\zeta_{H}\left(\varphi_{0}+s\right) & =\sum_{t \in T(K)} H\left(t,-s-\varphi_{0}\right) \\
& =\frac{[A(T)]}{(2 \pi)^{\operatorname{rg}\left(X(T)^{G}\right)} b(T)} \int_{y \in X(T)_{\mathbf{R}}^{G}} F\left(s-i \gamma_{\mathbf{R}}(y)\right) d y .
\end{aligned}
$$

où $F$ est la fonction définie dans l'énoncé de la proposition 5.46. En particulier $F$ est une fonction holomorphe sur $\mathcal{T}\left(\mathbf{R}_{>0}^{\Sigma(1) / G}\right)$, et il existe un $\varepsilon>0$ tel que la fonction

$$
s \mapsto F(s) \prod_{\alpha \in \Sigma(1) / G} \frac{s_{\alpha}}{1+s_{\alpha}}
$$

se prolonge en une fonction holomorphe sur $\mathcal{T}\left(\mathbf{R}_{>-\varepsilon}^{\Sigma(1) / G}\right)$ qui d'après le point 3 de la proposition 5.46 est $X(T)_{\mathbf{R}}^{G}$-contrôlée au sens de [CLTs, Définition 3.13].

D'après (5.27), on a

$$
C_{0}=\lim _{s \rightarrow 0} s^{[\Sigma(1) / G]} F\left(s \varphi_{0}\right) .
$$


On déduit alors du théorème 6.2 qu'il existe un $\varepsilon^{\prime}>0$ tel que la fonction

$$
R(\boldsymbol{s})=\zeta_{H}\left(\varphi_{0}+\boldsymbol{s}\right)-\frac{[A(T)] C_{0}}{b(T)} \Xi_{\pi\left(\mathbf{z}^{\Sigma(1) / G}\right), \pi\left(\mathbf{R}_{\geqslant 0}^{\Sigma(1) / G}\right)}(\pi(\boldsymbol{s})),
$$

se prolonge en une fonction méromorphe sur $\mathcal{T}\left(\mathbf{R}_{>-\varepsilon^{\prime}}^{\Sigma(1) / G}\right)$, vérifiant

$$
\forall \boldsymbol{s} \in \mathcal{T}\left(\mathbf{R}_{>0}^{\Sigma(1) / G}\right), \quad \lim _{s \rightarrow 0} s^{\operatorname{rg} \operatorname{Pic}\left(X_{\Sigma}\right)} R\left(s \varphi_{0}\right)=0
$$

La formule (6.3) et la suite exacte (8.1) montrent par ailleurs qu'on a

$$
\Xi_{\pi\left(\mathbf{z}^{\Sigma(1) / G}\right), \pi\left(\mathbf{R}_{\geqslant 0}^{\Sigma(1) / G}\right)}(\pi(\boldsymbol{s}))=\left[H^{1}(G, X(T))\right] \Xi_{\operatorname{Pic}\left(X_{\Sigma}\right), C_{\mathrm{eff}}\left(X_{\Sigma}\right)}(\pi(\boldsymbol{s}))+R(\boldsymbol{s}) .
$$

On en déduit que la fonction

$$
s \longmapsto \zeta_{\varphi_{0}}(s)=\zeta_{H}\left(s \varphi_{0}\right)
$$

prolonge en une fonction méromorphe sur $\mathcal{T}\left(\mathbf{R}_{>1-\varepsilon^{\prime}}\right)$ avec un pôle d'ordre $\operatorname{rg}\left(\operatorname{Pic}\left(X_{\Sigma}\right)\right)$ en $s=1$, de terme principal en $s=1$ égal à

$$
\left[H^{1}(G, X(T))\right] \alpha^{*}\left(X_{\Sigma}\right) \frac{[A(T)] C_{0}}{b(T)} .
$$

\subsection{Application du lemme technique dans le cas fonc- tionnel}

\subsubsection{Le cas d'une extension de déploiement non ramifiée}

On suppose dans toute la sous-section 8.3.1 que la variété torique $X_{\Sigma}$ est déployée par une extension non ramifiée.

Rappelons que d'après le corollaire 5.55 on a alors pour tout élément $\boldsymbol{s}$ de $\mathcal{T}\left(\mathbf{R}_{>1}^{\Sigma(1) / G}\right)$ la représentation intégrale

$$
\zeta_{H}(\boldsymbol{s})=\frac{1}{\log (q)^{\operatorname{rg}\left(X(T)^{G}\right)} b(T)} \sum_{\chi \in \mathcal{U}_{T}} I_{\chi}(\boldsymbol{s})
$$

où, pour $\chi \in \mathcal{U}_{T}, I_{\chi}(s)$ est la fonction donnée par l'intégrale

$$
\int_{\boldsymbol{z} \in X(T)_{U}^{G}}\left(\prod_{\alpha \in \Sigma(1) / G} \mathcal{L}_{K_{\alpha}}\left(\left\langle\boldsymbol{z}, d_{\alpha} \rho_{\alpha}\right\rangle q^{-d_{\alpha} s_{\alpha}}, \chi_{\alpha}\right)\right) \mathfrak{Q}\left(\chi, \gamma_{\boldsymbol{U}}(\boldsymbol{z}) q^{-\boldsymbol{s}}\right) d \boldsymbol{z} .
$$


Soit $P$ la série formelle

$$
\begin{aligned}
& P\left(\left(z_{\alpha}\right)\right)=\prod_{\alpha \in \Sigma(1) / G}\left(1-z_{\alpha}\right) \\
& \quad \times \sum_{\chi \in \mathcal{U}_{T}} \prod_{\alpha \in \Sigma(1) / G} \mathcal{L}_{K_{\alpha}}\left(q^{-d_{\alpha}} z_{\alpha}, \chi_{\alpha}\right) \prod_{v \in \mathcal{P}_{K}} Q_{\Sigma, v}\left(\chi_{\beta}\left(\pi_{w_{\beta}}\right) q^{-d_{\beta}} z_{\beta}^{f_{w_{\beta}}}\right) .
\end{aligned}
$$

D'après (2.6), le lemme 5.1 et le lemme $5.36, P$ a un rayon de convergence supérieur à $q^{\frac{1}{2}} ;$ en outre, pour tout $\boldsymbol{s} \in \mathcal{T}\left(\mathbf{R}_{>-\frac{1}{2}}^{\Sigma(1) / G}\right)$ et tout $\boldsymbol{z} \in X(T)_{\boldsymbol{U}}^{G}$, on a d'après le lemme 5.36 et le lemme 5.16

$$
\begin{aligned}
& \frac{P\left(\left\langle\boldsymbol{z}, d_{\alpha} \rho_{\alpha}\right\rangle q^{-\left(d_{\alpha} s_{\alpha}\right)}\right)}{\prod_{\alpha \in \Sigma(1) / G}\left(1-\left\langle\boldsymbol{z}, d_{\alpha} \rho_{\alpha}\right\rangle q^{\left.-d_{\alpha} s_{\alpha}\right)}\right.} \\
= & \sum_{\chi \in \mathcal{U}_{T}} \prod_{\alpha \in \Sigma(1) / G} \mathcal{L}_{K_{\alpha}}\left(\chi_{\alpha},\left\langle\boldsymbol{z}, d_{\alpha} \rho_{\alpha}\right\rangle q^{-d_{\alpha}\left(s_{\alpha}+1\right)}\right) \mathfrak{Q}\left(\chi, \gamma_{\boldsymbol{U}}(\boldsymbol{z}) q^{-\boldsymbol{s}-\varphi_{0}}\right) .
\end{aligned}
$$

On peut donc écrire

$$
\zeta_{H}\left(\boldsymbol{s}+\varphi_{0}\right)=\frac{1}{\log (q)^{\operatorname{rg}\left(X(T)^{G}\right)} b(T)} \int_{\boldsymbol{z} \in X(T)_{U}^{G}} \frac{P\left(\left\langle\boldsymbol{z}, d_{\alpha} \rho_{\alpha}\right\rangle q^{-d_{\alpha} s_{\alpha}}\right)_{\alpha \in \Sigma(1) / G}}{\prod_{\alpha \in \Sigma(1) / G}\left(1-\left\langle\boldsymbol{z}, d_{\alpha} \rho_{\alpha}\right\rangle q^{\left.-d_{\alpha} s_{\alpha}\right)}\right.} d \boldsymbol{z} .
$$

\section{Lemme 8.1}

On a

$$
P(1)=\log (q)^{[\Sigma(1) / G]}\left(\prod_{\alpha} d_{\alpha}\right) C_{0}
$$

Démonstration : Comme $X_{\Sigma}$ est déployée par une extension non ramifiée, d'après le corollaire 3.34 on a $\overline{T(K)} \cap T\left(\mathbf{A}_{K}\right)=T\left(\mathbf{A}_{K}\right)$. Ainsi on a

$$
C_{0}=\lim _{s \rightarrow 1}(s-1)^{[\Sigma(1) / G]} \mathcal{F} H\left(\mathbf{1},-s \varphi_{0}\right) .
$$

D'après (5.97) et la remarque qui suit, on a

$$
\mathcal{F} H\left(\mathbf{1},-s \varphi_{0}\right)=\left(\prod_{\alpha \in \Sigma(1) / G} Z_{K_{\alpha}}\left(\chi_{\alpha}, q^{-d_{\alpha} s}\right)\right) \mathfrak{Q}\left(\mathbf{1}, q^{-s \varphi_{0}}\right)
$$

d'où

$$
C_{0}=\lim _{s \rightarrow 0} s^{[\Sigma(1) / G]} \prod_{\alpha \in \Sigma(1) / G} Z_{K_{\alpha}}\left(q^{-d_{\alpha}(s+1)}\right) \mathfrak{Q}\left(\mathbf{1}, q^{-(s+1) \varphi_{0}}\right)
$$


D'après le lemme 5.41, $\operatorname{Ker}\left(\gamma^{*}\right)$ s'identifie à $A(T)^{*}$. Or, comme l'extension de déploiement $L / K$ est non ramifiée, d'après le corollaire $3.34 A(T)$ est trivial, donc $\operatorname{Ker}\left(\gamma^{*}\right)$ également. En particulier, si $\chi \in \mathcal{U}_{T} \backslash\{\mathbf{1}\}$, $\chi$ n'est pas dans $\operatorname{Ker}\left(\gamma^{*}\right)$, et donc l'un au moins des caractères $\chi_{\alpha}$ est non trivial. D'après le lemme 5.1, ceci montre l'égalité

$$
\begin{aligned}
& \lim _{s \rightarrow 0} s^{[\Sigma(1) / G]} \prod_{\alpha \in \Sigma(1) / G} Z_{K_{\alpha}}\left(q^{-d_{\alpha}(s+1)}\right) \mathfrak{Q}\left(\mathbf{1}, q^{-(s+1) \varphi_{0}}\right) \\
& =\lim _{s \rightarrow 0} s^{[\Sigma(1) / G]} \sum_{\chi \in \mathcal{U}_{T}} \prod_{\alpha \in \Sigma(1) / G} \mathcal{L}_{K_{\alpha}}\left(\chi_{\alpha}, q^{-d_{\alpha}\left(s_{\alpha}+1\right)}\right) \mathfrak{Q}\left(\chi, q^{(s+1) \varphi_{0}}\right) .
\end{aligned}
$$

Mais d'après (8.4), on a

$$
\begin{aligned}
& \lim _{s \rightarrow 0} s^{[\Sigma(1) / G]} \sum_{\chi \in \mathcal{U}_{T}} \prod_{\alpha \in \Sigma(1) / G} \mathcal{L}_{K_{\alpha}}\left(\chi_{\alpha}, q^{-d_{\alpha}(s+1)}\right) \mathfrak{Q}\left(\chi, q^{(s+1) \varphi_{0}}\right) . \\
& =\lim _{s \rightarrow 0} s^{[\Sigma(1) / G]} \frac{P\left(q^{-d_{\alpha} s}\right)}{\prod_{\alpha \in \Sigma(1) / G}\left(1-q^{-d_{\alpha} s}\right)}=P(1) \lim _{s \rightarrow 0} \frac{s^{[\Sigma(1) / G]}}{\prod_{\alpha \in \Sigma(1) / G}\left(1-q^{-d_{\alpha} s}\right)}
\end{aligned}
$$

d'où le résultat.

Reprenons à présent les notations de la section 7 . Le rôle de la suite exacte (7.1) est ici joué par la suite exacte

$$
0 \longrightarrow \mathcal{D}_{T_{\mathrm{NS}}}^{0} \stackrel{\pi^{\vee}}{\longrightarrow} \mathcal{D}_{T_{P_{\Sigma}}} \stackrel{\gamma^{\vee}}{\longrightarrow} \mathcal{D}_{T}^{0} \longrightarrow 0
$$

On pose $I=\Sigma(1) / G$, et on prend pour base $\left(\lambda_{i}\right)$ la base $\left(d_{\alpha} D_{\alpha}^{\vee}\right)$ de $\mathcal{D}_{T_{P_{\Sigma}}}$. On prend pour $a$ la fonction donnée par les coefficients de la série formelle $P$. On a donc, d'après (8.5) et le lemme 5.16

$$
\zeta_{H}\left(\varphi_{0}+\boldsymbol{s}\right)=\frac{1}{\log (q)^{\operatorname{rg}\left(X(T)^{G}\right)} b(T)} \int_{\boldsymbol{z} \in X(T)_{U}^{G}} \mathcal{L}_{N, \Lambda, a}\left(\gamma_{\boldsymbol{U}}(\boldsymbol{z}) q^{-\boldsymbol{s}}\right) \mathcal{L}_{N, \Lambda}\left(\gamma_{\boldsymbol{U}}(\boldsymbol{z}) q^{-\boldsymbol{s}}\right) d \boldsymbol{z}
$$

D'après le lemme 8.1 on a

$$
\mathcal{L}_{N, \Lambda, a}(1)=\log (q)^{[\Sigma(1) / G]}\left(\prod_{\alpha} d_{\alpha}\right) C_{0} \neq 0 .
$$

Nous appliquons alors le lemme 7.10 (ce qui est licite d'après (8.4)), en tenant compte de la remarque 7.9 et du fait que $X(T)^{G}$ est un sous-groupe d'indice fini de $\left(\mathcal{D}_{T}^{0}\right)^{\vee}$.

Nous obtenons ainsi le lemme suivant. 


\section{Lemme 8.2}

\section{La fonction}

$$
\boldsymbol{s} \mapsto \zeta_{H}\left(\boldsymbol{s}+\varphi_{0}\right)-\frac{\log (q)^{[\Sigma(1) / G]}\left(\prod_{\alpha} d_{\alpha}\right) C_{0}}{\log (q)^{\operatorname{rg}\left(X(T)^{G}\right)} b(T)} \mathcal{L}_{\mathcal{D}_{T_{\mathrm{NS}}}^{0}, \Lambda \cap \mathcal{D}_{T_{\mathrm{NS}}}^{0}}\left(q^{-\boldsymbol{s}}\right)
$$

est admissible de multiplicité supérieure à $1-\operatorname{rg}\left(\operatorname{Pic}\left(X_{\Sigma}\right)\right)$.

\section{Lemme 8.3}

Il existe un $\varepsilon>0$ tel que la fonction

$$
s \mapsto \zeta_{H}\left(s \phi_{0}\right)
$$

se prolonge en une fonction méromorphe sur $\mathcal{T}\left(\boldsymbol{R}_{>1-\varepsilon}\right)$ avec un pôle d'ordre $\operatorname{rg}\left(\operatorname{Pic}\left(X_{\Sigma}\right)\right)$ en $s=0$, et vérifiant

$$
\lim _{s \rightarrow 0} s^{\operatorname{rg}\left(\operatorname{Pic}\left(X_{\Sigma}\right)\right)} \zeta_{H}\left(s \varphi_{0}\right)=\left[H^{1}(G, X(T))\right]\left[\mathcal{C}_{T}\right] \frac{[A(T)] C_{0}}{b(T)} \alpha^{*}\left(X_{\Sigma}\right) .
$$

Démonstration : Du lemme 8.2, on déduit aussitôt qu'il existe un $\varepsilon>0$ tel que la fonction $s \mapsto \zeta_{H}\left(s \varphi_{0}\right)$ se prolonge en une fonction méromorphe sur $\mathcal{T}\left(\mathbf{R}_{>1-\varepsilon}\right)$ avec un pôle d'ordre $\operatorname{rg}\left(\operatorname{Pic}\left(X_{\Sigma}\right)\right)$ en $s=0$, et vérifiant

$$
\begin{aligned}
\lim _{s \rightarrow 0} s^{\operatorname{rg}\left(\operatorname{Pic}\left(X_{\Sigma}\right)\right)} \zeta_{H}\left(s \varphi_{0}\right)= & \log (q)^{\operatorname{rg}\left(\operatorname{Pic}\left(X_{\Sigma}\right)\right)}\left(\prod_{\alpha} d_{\alpha}\right) C_{0} \frac{1}{b(T)} \\
& \times \lim _{s \rightarrow 0}\left[s^{\operatorname{rg}\left(\operatorname{Pic}\left(X_{\Sigma}\right)\right)} \mathcal{L}_{\left.\mathcal{D}_{T_{\mathrm{NS}}}^{0}, \Lambda \cap \mathcal{D}_{T_{\mathrm{NS}}}^{0}\left(q^{-s \varphi_{0}}\right)\right] .}\right.
\end{aligned}
$$

Mais on a

$$
\begin{aligned}
\lim _{s \rightarrow 0} s^{\operatorname{rg}\left(\operatorname{Pic}\left(X_{\Sigma}\right)\right)} \mathcal{L}_{\mathcal{D}_{T_{\mathrm{NS}}}^{0}, \Lambda \cap \mathcal{D}_{T_{\mathrm{NS}}}^{0}}\left(q^{-s \varphi_{0}}\right) \\
=\frac{\left[H^{1}(G, X(T))\right]\left[\mathcal{C}_{T}\right]}{\prod_{\alpha} d_{\alpha}} \log (q)^{-\operatorname{rg}\left(\operatorname{Pic}\left(X_{\Sigma}\right)\right)} \alpha^{*}\left(X_{\Sigma}\right) .
\end{aligned}
$$

La relation (8.19) sera démontré à la sous-section suivante (démonstration du lemme 8.7) dans le cas où l'extension de déploiement n'est plus supposée non ramifiée. Supposer l'extension de déploiement non ramifiée ne simplifie pas notoirement le calcul (on y gagne simplement le fait que certains termes apparaissant dans le calcul, comme $[A(T)]$ et $\mathcal{K}_{T}$, sont triviaux). 


\subsubsection{Un cas plus général}

On suppose dans toute la sous-section 8.3.2, que le G-éventail $\Sigma$ est tel que toutes les places de $K$ vérifient l'hypothèse 4.8. Nous expliquerons en appendice comment adapter le raisonnement au cas général.

Rappelons que d'après le corollaire 5.54 on a alors pour tout élément $s$ de $\mathcal{T}\left(\mathbf{R}_{>1}^{\Sigma(1) / G}\right)$ la représentation intégrale

$$
\zeta_{H}(\boldsymbol{s})=\frac{1}{\log (q)^{\operatorname{rg}\left(X(T)^{G}\right)} b(T)}\left(\frac{[A(T)]}{\left[\mathcal{K}_{T}\right]} I_{1}(\boldsymbol{s})+\sum_{\chi \in \mathcal{U}_{T} \backslash \operatorname{Ker}\left(\gamma^{*}\right)} I_{\chi}(\boldsymbol{s})\right)
$$

où $I_{1}(\boldsymbol{s})$ est la fonction donnée par l'intégrale

$$
\int_{\boldsymbol{z} \in X(T) G}\left(\prod_{U \in \Sigma(1) / G} Z_{K_{\alpha}}\left(\left\langle\boldsymbol{z}, d_{\alpha} \rho_{\alpha}\right\rangle q^{-d_{\alpha} s_{\alpha}}\right)\right) \mathfrak{f}\left(\gamma_{\boldsymbol{U}}(\boldsymbol{z}) q^{-\boldsymbol{s}}\right) d \boldsymbol{z}
$$

et $I_{\chi}(s)$ est la fonction donnée par l'intégrale

$$
\int_{\boldsymbol{z} \in X(T)_{U}^{G}}\left(\prod_{\alpha \in \Sigma(1) / G} \mathcal{L}_{K_{\alpha}}\left(\chi_{\alpha},\left\langle\boldsymbol{z}, d_{\alpha} \rho_{\alpha}\right\rangle q^{-d_{\alpha} s_{\alpha}}\right)\right) \mathfrak{f}\left(\chi, \gamma_{\boldsymbol{U}}(\boldsymbol{z}) q^{-\boldsymbol{s}}\right) d \boldsymbol{z} .
$$

Étude de $\boldsymbol{I}_{\chi}$ pour $\chi \in \mathcal{U}_{\boldsymbol{T}} \backslash \operatorname{Ker}\left(\gamma^{*}\right)$. Soit $\chi \in \mathcal{U}_{T} \backslash \operatorname{Ker}\left(\gamma^{*}\right)$. Étudions l'intégrale

$$
I_{\chi}(\boldsymbol{s})=\int_{\boldsymbol{z} \in X(T)_{U}^{G}}\left(\prod_{\alpha \in \Sigma(1) / G} \mathcal{L}_{K_{\alpha}}\left(\chi_{\alpha},\left\langle\boldsymbol{z}, d_{\alpha} \rho_{\alpha}\right\rangle q^{-d_{\alpha} s_{\alpha}}\right)\right) \mathfrak{f}\left(\chi, \gamma_{\boldsymbol{U}}(\boldsymbol{z}) q^{-\boldsymbol{s}}\right) d \boldsymbol{z} .
$$

Rappelons que d'après le lemme 5.37 la série $\mathfrak{f}(\chi,$.$) est une série formelle$ de rayon de convergence supérieur à $q^{-\frac{1}{2}}$ et $\mathcal{D}_{T}$-compatible.

Comme $\chi$ n'est pas dans $\operatorname{Ker}\left(\gamma^{*}\right)$ l'ensemble $(\Sigma(1) / G)_{\chi}$ des $\alpha \in \Sigma(1) / G$ tel que $\chi_{\alpha}$ est non trivial est non vide, et, par le lemme 5.1, pour de tels $\alpha$ la fonction $\mathcal{L}_{K_{\alpha}}\left(\chi_{\alpha},.\right)$ est un polynôme. Pour les autres $\alpha$ la fonction $\mathcal{L}$ correspondante est une fonction zêta. 
Ceci montre, compte tenu également de la proposition 2.1, que la série

$$
\begin{aligned}
P\left(\chi,\left(z_{\alpha}\right)\right) \stackrel{\text { déf }}{=} & \left(\prod_{\alpha \notin(\Sigma(1) / G)_{\chi}}\left(1-z_{\alpha}^{d_{\alpha}}\right) Z_{K_{\alpha}}\left(q^{-d_{\alpha}} z_{\alpha}^{d_{\alpha}}\right)\right) \\
& \times\left(\prod_{\alpha \in(\Sigma(1) / G)_{\chi}} \mathcal{L}_{K_{\alpha}}\left(\chi_{\alpha}, q^{-d_{\alpha}} z_{\alpha}^{d_{\alpha}}\right)\right) \mathfrak{f}\left(\chi,\left(q^{-1} z_{\alpha}\right)\right)
\end{aligned}
$$

a un rayon de convergence supérieur à $q^{\frac{1}{2}}$ et est $\mathcal{D}_{T}$-compatible. On a pour tout $\boldsymbol{s} \in \mathcal{T}\left(\mathbf{R}_{>0}^{\Sigma(1) / G}\right)$ et tout $\boldsymbol{z} \in X(T)_{\boldsymbol{U}}^{G}$

$$
\begin{aligned}
P & \left(\chi, \gamma_{\boldsymbol{U}}(\boldsymbol{z}) q^{-\boldsymbol{s}}\right) \\
& =\left(\prod_{\alpha \notin(\Sigma(1) / G)_{\chi}}\left(1-\left\langle\boldsymbol{z}, d_{\alpha} \rho_{\alpha}\right\rangle q^{-d_{\alpha} s_{\alpha}}\right) Z_{K_{\alpha}}\left(\left\langle\boldsymbol{z}, d_{\alpha} \rho_{\alpha}\right\rangle q^{-d_{\alpha}\left(s_{\alpha}+1\right)}\right)\right) \\
& \times\left(\prod_{\alpha \in(\Sigma(1) / G)_{\chi}} \mathcal{L}_{K_{\alpha}}\left(\chi_{\alpha},\left\langle\boldsymbol{z}, d_{\alpha} \rho_{\alpha}\right\rangle q^{-d_{\alpha}\left(s_{\alpha}+1\right)}\right)\right) \mathfrak{f}\left(\chi, \gamma_{\boldsymbol{U}}(\boldsymbol{z}) q^{-\left(\boldsymbol{s}+\varphi_{0}\right)}\right)
\end{aligned}
$$

On a donc

$$
I_{\chi}\left(s+\varphi_{0}\right)=\int_{\boldsymbol{z} \in X(T)_{U}^{G}} \frac{P\left(\chi, \gamma_{\boldsymbol{U}}(\boldsymbol{z}) q^{-\boldsymbol{s}}\right)_{\alpha \in \Sigma(1) / G}}{\prod_{\alpha \notin(1) / G)_{\chi}}\left(1-\left\langle\boldsymbol{z}, d_{\alpha} \rho_{\alpha}\right\rangle q^{-d_{\alpha} s_{\alpha}}\right)} d \boldsymbol{z} .
$$

Reprenons les notations de la section 7. Le rôle de la suite exacte (7.1) est ici joué par la suite exacte

$$
0 \longrightarrow\left(\pi\left(P_{\Sigma}^{G}\right)\right)^{\vee} \stackrel{\pi^{\vee}}{\longrightarrow}\left(P_{\Sigma}^{G}\right)^{\vee} \stackrel{\gamma^{\vee}}{\longrightarrow}\left(X(T)^{G}\right)^{\vee} \longrightarrow 0 .
$$

On pose $N^{\prime}=\mathcal{D}_{T_{P_{\Sigma}}}, I=\Sigma(1) / G, \widetilde{I}=(\Sigma(1) / G) \backslash(\Sigma(1) / G)_{\chi}$, et on prend pour base $\left(\lambda_{i}\right)$ de $N$ la base $\left(D_{\alpha}^{\vee}\right)_{\alpha \in \Sigma(1) / G}$. On prend pour $a$ la fonction donnée par les coefficients de la série formelle $P(\chi,$.$) . On peut alors écrire$

$$
I_{\chi}\left(\boldsymbol{s}+\varphi_{0}\right)=\int_{\boldsymbol{z} \in X(T)_{U}^{G}} \mathcal{L}_{N, \Lambda, a}\left(\gamma_{\boldsymbol{U}}(\boldsymbol{z}) q^{-\boldsymbol{s}}\right) \mathcal{L}_{N^{\prime}, \widetilde{\Lambda}}\left(\gamma_{\boldsymbol{U}}(\boldsymbol{z}) q^{-\boldsymbol{s}}\right) d \boldsymbol{z}
$$

On applique alors le lemme 7.14 et on obtient ainsi le lemme suivant. 


\section{Lemme 8.4}

\section{La fonction}

$$
s \mapsto I_{\chi}\left(s+\varphi_{0}\right)
$$

est une fonction admissible de multiplicité au plus

$$
[\Sigma(1) / G]-\operatorname{rg}\left(X(T)^{G}\right)-1=\operatorname{rg}\left(\operatorname{Pic}\left(X_{\Sigma}\right)\right)-1 .
$$

En particulier, il existe un $\varepsilon>0$, tel que la fonction $s \mapsto I_{\chi}\left((s+1) \varphi_{0}\right)$ se prolonge en une fonction méromorphe sur $\mathcal{T}\left(\boldsymbol{R}_{>-\varepsilon}\right)$, avec un pôle d'ordre au plus $\operatorname{rg}\left(\operatorname{Pic}\left(X_{\Sigma}\right)\right)-1$ en zéro.

Étude de $\boldsymbol{I}_{\mathbf{1}}$. Pour mémoire, $I_{1}(\boldsymbol{s})$ est donnée par l'intégrale

$$
\int_{\boldsymbol{z} \in X(T)_{U}^{G}}\left(\prod_{\alpha \in \Sigma(1) / G} Z_{K_{\alpha}}\left(\left\langle\boldsymbol{z}, d_{\alpha} \rho_{\alpha}\right\rangle q^{-d_{\alpha} s_{\alpha}}\right)\right) \mathfrak{f}\left(\gamma_{\boldsymbol{U}}(\boldsymbol{z}) q^{-\boldsymbol{s}}\right) d \boldsymbol{z} .
$$

Soit $P$ la série formelle

$$
P(\boldsymbol{z}) \stackrel{\text { déf }}{=}\left(\prod_{\alpha \in \Sigma(1) / G}\left(1-q^{-d_{\alpha}} z_{\alpha}^{d_{\alpha}}\right) Z_{K_{\alpha}}\left(q^{-d_{\alpha}} z_{\alpha}^{d_{\alpha}}\right)\right) \times \mathfrak{f}\left(\left(q^{-1} z_{\alpha}\right)\right) .
$$

D'après (2.6), le lemme 5.39 et le lemme $5.19 P$ est une série formelle de rayon de convergence supérieur à $q^{\frac{1}{2}}$ et $\mathcal{D}_{T}^{0}$-compatible. On a, pour tout $\boldsymbol{s} \in \mathcal{T}\left(\mathbf{R}_{>-\frac{1}{2}}^{\Sigma(1) / G}\right)$ et tout $\boldsymbol{z} \in X(T)_{U}^{G}$

$$
\begin{aligned}
& \frac{P\left(\gamma_{\boldsymbol{U}}(\boldsymbol{z}) q^{-\boldsymbol{s}}\right)}{\prod_{\alpha \in \Sigma(1) / G}\left(1-\left\langle\boldsymbol{z}, d_{\alpha} \rho_{\alpha}\right\rangle q^{-d_{\alpha} s_{\alpha}}\right)} \\
& \quad=\left(\prod_{\alpha \in \Sigma(1) / G} Z_{K_{\alpha}}\left(\left\langle\boldsymbol{z}, d_{\alpha} \rho_{\alpha}\right\rangle q^{-d_{\alpha}\left(s_{\alpha}+1\right)}\right)\right) \mathfrak{f}\left(\gamma_{\boldsymbol{U}}(\boldsymbol{z}) q^{-\left(\boldsymbol{s}+\varphi_{0}\right)}\right) .
\end{aligned}
$$

On a ainsi, pour tout $s \in \mathcal{T}\left(\mathbf{R}_{>-\frac{1}{2}}^{\Sigma(1) / G}\right)$

$$
I_{1}\left(\boldsymbol{s}+\varphi_{0}\right)=\int_{\boldsymbol{z} \in X(T)_{U}^{G}} \frac{P\left(\gamma_{\boldsymbol{U}}(\boldsymbol{z}) q^{-\boldsymbol{s}}\right)_{\alpha \in \Sigma(1) / G}}{\prod_{\alpha \in \Sigma(1) / G}\left(1-\left\langle\boldsymbol{z}, d_{\alpha} \rho_{\alpha}\right\rangle q^{-d_{\alpha} s_{\alpha}}\right)} d \boldsymbol{z} .
$$




\section{Lemme 8.5}

On a

$$
P(1)=\log (q)^{[\Sigma(1) / G]}\left(\prod_{\alpha} d_{\alpha}\right) C_{0}
$$

Démonstration : D'après le lemme 5.40, on a pour tout $s \in \mathcal{T}\left(\mathbf{R}_{>0}\right)$

$\left.\int_{\overline{T(K)} \cap T\left(\mathbf{A}_{K}\right)} H\left(-(s+1) \varphi_{0}\right), t\right) \omega_{T}(t)=\left(\prod_{\alpha \in \Sigma(1) / G} Z_{K_{\alpha}}\left(q^{-d_{\alpha}(s+1)}\right)\right) \mathfrak{f}\left(q^{-(s+1) \varphi_{0}}\right)$.

Par ailleurs on a, d'après (8.33),

$$
\left(\prod_{\alpha \in \Sigma(1) / G} Z_{K_{\alpha}}\left(q^{-d_{\alpha}(s+1)}\right)\right) \mathfrak{f}\left(q^{-(s+1) \varphi_{0}}\right)=\frac{P\left(q^{-s \varphi_{0}}\right)}{\prod_{\alpha \in \Sigma(1) / G}\left(1-q^{-d_{\alpha} s}\right)}
$$

On en déduit qu'on a

$$
\begin{aligned}
C_{0} & \left.=\lim _{s \rightarrow 0} s^{[\Sigma(1) / G]} \int_{\overline{T(K)} \cap T\left(\mathbf{A}_{K}\right)} H\left(-(s+1) \varphi_{0}\right), t\right) \omega_{T}(t) \\
& =\lim _{s \rightarrow 0} s^{[\Sigma(1) / G]} \frac{P\left(q^{-s \varphi_{0}}\right)}{\prod_{\alpha \in \Sigma(1) / G}\left(1-q^{-d_{\alpha} s}\right)} \\
& =P(1) \lim _{s \rightarrow 0} \frac{s^{[\Sigma(1) / G]}}{\prod_{\alpha \in \Sigma(1) / G}\left(1-q^{-d_{\alpha} s}\right)}
\end{aligned}
$$

d'où le résultat annoncé.

Reprenons les notations de la section 7 . Le rôle de la suite exacte (7.1) est ici joué par la suite exacte

$$
0 \longrightarrow \mathcal{D}_{T_{\mathrm{NS}}}^{0} \stackrel{\pi^{\vee}}{\longrightarrow} \mathcal{D}_{T_{P_{\Sigma}}} \stackrel{\gamma^{\vee}}{\longrightarrow} \mathcal{D}_{T}^{0} \longrightarrow 0 .
$$

On pose $I=\Sigma(1) / G$, et on prend pour base $\left(\lambda_{i}\right)$ la base $\left(d_{\alpha} D_{\alpha}^{\vee}\right)$ de $\mathcal{D}_{T_{P_{\Sigma}}}$. On prend pour $a$ la fonction donnée par les coefficients de la série formelle $P$. On peut alors écrire

$$
I_{1}\left(\boldsymbol{s}+\varphi_{0}\right)=\int_{\boldsymbol{z} \in X(T)_{U}^{G}} \mathcal{L}_{N, \Lambda, a}\left(\gamma_{\boldsymbol{U}}(\boldsymbol{z}) q^{-\boldsymbol{s}}\right) \mathcal{L}_{N^{\prime}, \Lambda}\left(\gamma_{\boldsymbol{U}}(\boldsymbol{z}) q^{-\boldsymbol{s}}\right) d \boldsymbol{z}
$$


D'après le lemme 8.1 on a

$$
\mathcal{L}_{N, \Lambda, a}(1)=\log (q)^{[\Sigma(1) / G]}\left(\prod_{\alpha} d_{\alpha}\right) C_{0} \neq 0 .
$$

Comme on a $j\left(N^{\prime}\right)=\gamma^{\vee}\left(\mathcal{D}_{T_{P_{\Sigma}}}\right)=\mathcal{D}_{T}^{0}$, d'après la définition 5.17, le fait que $P$ soit $\mathcal{D}_{T}^{0}$-compatible signifie que $a$ vérifie la condition suivante : si $a_{y}$ est non nul alors $j(y)$ est un élément de $j\left(N^{\prime}\right)$.

Nous appliquons alors le lemme 7.12 (ce qui est licite d'après (8.4)) en tenant compte de la remarque 7.11 et du fait que $X(T)^{G}$ est un sous-groupe d'indice fini de $\left(\mathcal{D}_{T}^{0}\right)^{\vee}$. Nous obtenons ainsi le lemme suivant.

\section{Lemme 8.6}

Il existe une fonction admissible $g$ de multiplicité supérieure à zéro et vérifiant

$$
g(0)=\log q^{[\Sigma(1) / G]}\left(\prod_{\alpha} d_{\alpha}\right) C_{0}
$$

telle que la fonction

$$
\boldsymbol{s} \mapsto I_{1}\left(\boldsymbol{s}+\varphi_{0}\right)-g(\boldsymbol{s}) \mathcal{L}_{\mathcal{D}_{T_{\mathrm{NS}}}^{0}, \Lambda \cap \mathcal{D}_{T_{\mathrm{NS}}}^{0}}\left(q^{-\boldsymbol{s}}\right)
$$

est admissible de multiplicité supérieure à $1-\operatorname{rg}\left(\operatorname{Pic}\left(X_{\Sigma}\right)\right)$.

\section{Conclusion}

\section{Lemme 8.7}

Il existe un $\varepsilon>0$ tel que la fonction

$$
s \mapsto \zeta_{H}\left(s \varphi_{0}\right)
$$

se prolonge en une fonction méromorphe sur $\mathcal{T}\left(\boldsymbol{R}_{>1-\varepsilon}\right)$ avec un pôle d'ordre $\operatorname{rg}\left(\operatorname{Pic}\left(X_{\Sigma}\right)\right)$ en $s=0$, et vérifiant

$$
\lim _{s \rightarrow 0} s^{\operatorname{rg}\left(\operatorname{Pic}\left(X_{\Sigma}\right)\right)} \zeta_{H}\left(s \varphi_{0}\right)=\left[H^{1}(G, X(T))\right]\left[\mathcal{C}_{T}\right] \frac{[A(T)] C_{0}}{b(T)} \alpha^{*}\left(X_{\Sigma}\right) .
$$

Démonstration : Des lemmes 8.4, 8.6, et de (8.20), on déduit aussitôt qu'il existe un $\varepsilon>0$ tel que la fonction $s \mapsto \zeta_{H}\left(s \varphi_{0}\right)$ se prolonge en une fonction méromorphe sur $\mathcal{T}\left(\mathbf{R}_{>1-\varepsilon}\right)$ avec un pôle d'ordre $\operatorname{rg}\left(\operatorname{Pic}\left(X_{\Sigma}\right)\right)$ en 
$s=0$, et vérifiant

$$
\begin{aligned}
& \lim _{s \rightarrow 0} s^{\operatorname{rg}\left(\operatorname{Pic}\left(X_{\Sigma}\right)\right)} \zeta_{H}\left(s \varphi_{0}\right) \\
= & \frac{[A(T)] \log (q)^{[\Sigma(1) / G]}\left(\prod_{\alpha} d_{\alpha}\right) C_{0}}{\mathcal{K}_{T} \log (q)^{\operatorname{rg}\left(X(T)^{G}\right)} b(T)} \lim _{s \rightarrow 0}\left[s^{\operatorname{rg}\left(\operatorname{Pic}\left(X_{\Sigma}\right)\right)} \mathcal{L}_{\left.\mathcal{D}_{T_{\mathrm{NS}}}^{0}, \Lambda \cap \mathcal{D}_{T_{\mathrm{NS}}}^{0}\left(q^{-s \varphi_{0}}\right)\right] .}\right.
\end{aligned}
$$

Mais on a

$$
\begin{aligned}
& \lim _{s \rightarrow 0} s^{\operatorname{rg}\left(\operatorname{Pic}\left(X_{\Sigma}\right)\right)} \mathcal{L}_{\mathcal{D}_{T_{\mathrm{NS}}}^{0}, \Lambda \cap \mathcal{D}_{T_{\mathrm{NS}}}^{0}}\left(q^{-s \varphi 0}\right) \\
= & \frac{\left[H^{1}(G, X(T))\right]\left[\mathcal{C}_{T_{\mathrm{NS}}}\right]\left[\mathcal{C}_{T}\right] \mathcal{K}_{T}}{\left[\mathcal{C}_{T_{P_{\Sigma}}}\right]} \lim _{s \rightarrow 0} s^{\operatorname{rg}\left(\operatorname{Pic}\left(X_{\Sigma}\right)\right)} \mathcal{L}_{\gamma^{\vee}\left(\mathcal{D}_{T_{\mathrm{NS}}}\right), \Lambda \cap \gamma^{\vee}\left(\mathcal{D}_{T_{\mathrm{NS}}}\right)}\left(q^{-s \varphi_{0}}\right) \\
= & \frac{\left[H^{1}(G, X(T))\right]\left[\mathcal{C}_{T}\right] \mathcal{K}_{T}}{\left[\mathcal{C}_{T_{P_{\Sigma}}}\right]} \lim _{s \rightarrow 0} s^{\operatorname{rg}\left(\operatorname{Pic}\left(X_{\Sigma}\right)\right)} \mathcal{L}_{\operatorname{Pic}\left(X_{\Sigma}\right)^{\vee}, C_{\mathrm{eff}}\left(X_{\Sigma}\right)^{\vee}\left(q^{-s \gamma\left[\varphi_{0}\right]}\right)} \\
= & \frac{\left[H^{1}(G, X(T))\right]\left[\mathcal{C}_{T}\right] \mathcal{K}_{T}}{\left[\mathcal{C}_{T_{P_{\Sigma}}}\right]} \log (q)^{-\operatorname{rg}\left(\operatorname{Pic}\left(X_{\Sigma}\right)\right)} \alpha^{*}\left(X_{\Sigma}\right) .
\end{aligned}
$$

La première égalité vient de (7.4) et (8.8), la seconde de (7.4) et du fait que, par définition, $\mathcal{D}_{T_{\mathrm{NS}}}$ est d'indice $\left[\mathcal{C}_{T_{\mathrm{NS}}}\right]$ dans $\operatorname{Pic}\left(X_{\Sigma}\right)^{\vee}$, et la dernière égalité vient de (7.3). Comme on a $\left[\mathrm{C}_{T_{P_{\Sigma}}}\right]=\prod_{\alpha} d_{\alpha}$ et

$$
\operatorname{rg}\left(\operatorname{Pic}\left(X_{\Sigma}\right)\right)+\operatorname{rg}\left(X(T)^{G}\right)=[\Sigma(1) / G]
$$

on en déduit le résultat annoncé.

\subsection{Calcul du terme principal de la fonction zêta des hauteurs}

\subsubsection{Calcul de $C_{0}$}

Nous calculons à présent la constante $C_{0}$ définie en (8.9) (et montrons en particulier qu'elle est non nulle). 


\section{Lemme 8.8}

On a

$$
\begin{aligned}
& C_{0}=\lim _{s \rightarrow 1}(s-1)^{[\Sigma(1) / G]} \int_{\overline{T(K)} \cap T\left(\boldsymbol{A}_{K}\right)} H\left(-s \varphi_{0}, t\right) \omega_{T}(t) \\
&=\ell(X(T)) \gamma_{H}\left(X_{\Sigma}\right) .
\end{aligned}
$$

Démonstration : La première égalité est la définition de $C_{0}$. Montrons la deuxième égalité. D'après le scindage

$$
\overline{T(K)} \cap T\left(\mathbf{A}_{K}\right)=\overline{T(K)}^{S} \times T\left(\mathbf{A}_{K}\right)^{S}
$$

donné par le lemme 3.32 et la définition (3.6) de $\omega_{T}$ on peut écrire pour tout $s \in \mathcal{T}\left(\mathbf{R}_{>1}\right)$

$$
\begin{aligned}
& \int_{\overline{T(K)} \cap T\left(\mathbf{A}_{K}\right)} H(-(s, \ldots, s), t) \omega_{T} \\
& =c_{K, \operatorname{dim}\left(X_{\Sigma}\right)} \int_{\frac{T(K)}{S}}\left(\prod_{v \in S} H_{v}(-(s, \ldots, s), t)\right) \underset{v \in S}{\otimes} d \mu_{v} \\
& \times \prod_{v \notin S} H_{v}(-(s, \ldots, s), t) d \mu_{v} .
\end{aligned}
$$

D'après le lemme 5.29 , on en déduit

$$
\begin{aligned}
& \int_{\overline{T(K)} \cap T\left(\mathbf{A}_{K}\right)} H(-(s, \ldots, s), t) \omega_{T} \\
& =c_{K, \operatorname{dim}\left(X_{\Sigma}\right)} \int_{\frac{T(K)}{T(K)}}\left(\prod_{v \in S} H_{v}(-(s, \ldots, s), t)\right) \underset{v \in S}{\otimes} d \mu_{v} \\
& \times L_{S}\left(s, P_{\Sigma}\right) \prod_{v \notin S} Q_{\Sigma, v}\left(q_{v}^{-l_{\beta} s}\right)_{\beta \in \Sigma(1) / G_{v}} .
\end{aligned}
$$


On en tire

$$
\begin{aligned}
\lim _{s \rightarrow 1}(s-1)^{[\Sigma(1) / G]} \int_{\overline{T(K)} \cap T\left(\mathbf{A}_{K}\right)} H(-(s, \ldots, s), t) \omega_{T} \\
=\frac{\ell\left(P_{\Sigma}\right)}{\prod_{v \in S} L_{v}\left(1, P_{\Sigma}\right)} c_{K, \operatorname{dim}\left(X_{\Sigma}\right)} \int_{\frac{T(K)}{T(K)}}\left(\prod_{v \in S} H_{v}(-(1, \ldots, 1), t)\right) \underset{v \in S}{\otimes} d \mu_{v} \\
\times \prod_{v \notin S} Q_{\Sigma, v}\left(q_{v}^{-l_{\beta}}\right)_{\beta \in \Sigma(1) / G_{v}} .
\end{aligned}
$$

Par ailleurs, toujours d'après le lemme 5.29, on a pour $v \notin S$

$$
\begin{aligned}
Q_{\Sigma, v}\left(q_{v}^{-l_{\beta}}\right) & =L_{v}\left(1, P_{\Sigma}\right)^{-1} \int_{T\left(K_{v}\right)} H_{v}(-(1, \ldots, 1), t) d \mu_{v} \\
& =\frac{L_{v}(1, X(T))}{L_{v}\left(1, P_{\Sigma}\right)} \int_{T\left(K_{v}\right)} H_{v}(-(1, \ldots, 1), t) \omega_{T, v},
\end{aligned}
$$

la deuxième égalité provenant de la définition (3.3) de $d \mu_{v}$. D'après le lemme 2.4 et la suite exacte (4.11), on a donc

$$
Q_{\Sigma, v}\left(q_{v}^{-l_{\beta}}\right)=\int_{T\left(K_{v}\right)} H_{v}(-(1, \ldots, 1), t) \frac{\omega_{T, v}}{L_{v}\left(1, \operatorname{Pic}\left(X_{\Sigma, L}\right)\right)}
$$

On en déduit que la limite

$$
\lim _{s \rightarrow 1}(s-1)^{[\Sigma(1) / G]} \int_{\overline{T(K)} \cap T\left(\mathbf{A}_{K}\right)} H(-(s, \ldots, s), t) \omega_{T}
$$

est égale à

$$
\begin{aligned}
\ell\left(P_{\Sigma}\right) & \frac{\prod_{v \in S} L_{v}(1, X(T)) L_{v}\left(1, \operatorname{Pic}\left(X_{\Sigma, L}\right)\right)}{\prod_{v \in S} L_{v}\left(1, P_{\Sigma}\right)} \\
\times c_{K, \operatorname{dim}\left(X_{\Sigma}\right) \int_{\frac{1}{T(K)}}} & \left(\prod_{v \in S} H_{v}(-(1, \ldots, 1), t)\right) \otimes_{v \in S} \frac{\omega_{T, v}}{L_{v}\left(1, \operatorname{Pic}\left(X_{\Sigma, L}\right)\right)} \\
\times & \prod_{v \notin S_{T\left(K_{v}\right)}} \int_{v} H_{v}(-(1, \ldots, 1), t) \frac{\omega_{T, v}}{L_{v}\left(1, \operatorname{Pic}\left(X_{\Sigma, L}\right)\right)}
\end{aligned}
$$


D'après le lemme 4.10 , on a

$$
\begin{array}{r}
c_{K, \operatorname{dim}\left(X_{\Sigma}\right)} \int_{\frac{T}{T(K)}}\left(\prod_{v \in S} H_{v}(-(1, \ldots, 1), x)\right) \otimes_{v \in S} \frac{\omega_{T, v}}{L_{v}\left(1, \operatorname{Pic}\left(X_{\Sigma, L}\right)\right)} \\
\prod_{v \notin S_{T\left(K_{v}\right)}} \int_{K_{v}} H_{v}(-(1, \ldots, 1), x) \frac{\omega_{T, v}}{L_{v}\left(1, \operatorname{Pic}\left(X_{\Sigma, L}\right)\right)} \\
=c_{K, \operatorname{dim}\left(X_{\Sigma}\right)} \int_{\frac{T(K)}{T}} \otimes_{v \in S} \frac{\omega_{X_{\Sigma}, v}}{L_{v}\left(1, \operatorname{Pic}\left(X_{\Sigma, L}\right)\right)} \prod_{v \notin S_{T\left(K_{v}\right)}} \int_{L_{v}\left(1, \operatorname{Pic}\left(X_{\Sigma, L}\right)\right)}
\end{array}
$$

En utilisant encore une fois le scindage (8.2) et la définition (4.11) de $\omega_{X_{\Sigma}}$ on trouve

$$
\begin{array}{r}
c_{K, \operatorname{dim}\left(X_{\Sigma}\right)} \int_{\frac{T}{T(K)}} \otimes_{v \in S} \frac{\omega_{X_{\Sigma}, v}}{L_{v}\left(1, \operatorname{Pic}\left(X_{\Sigma, L}\right)\right)} \times \prod_{v \notin S_{T\left(K_{v}\right)}} \int_{\frac{\omega_{X_{\Sigma}, v}}{L_{v}\left(1, \operatorname{Pic}\left(X_{\Sigma, L}\right)\right)}} \\
=\ell\left(\operatorname{Pic}\left(X_{\Sigma, L}\right)\right)^{-1} \int_{\overline{T(K)} \cap T\left(\mathbf{A}_{K}\right)} \omega_{X_{\Sigma}} .
\end{array}
$$

D'après le lemme 4.11 on a

$$
\int_{\overline{T(K)} \cap T\left(\mathbf{A}_{K}\right)} \omega_{X_{\Sigma}}=\int_{\frac{X_{\Sigma}(K)}{X_{X}}} \omega_{X_{\Sigma}} .
$$

Finalement, on trouve

$$
\begin{aligned}
\lim _{s \rightarrow 1}(s-1)^{[\Sigma(1) / G]} \int_{\overline{T(K)} \cap T\left(\mathbf{A}_{K}\right)} H(-(s, \ldots, s), x) \omega_{T} \\
=\frac{\ell\left(P_{\Sigma}\right)}{\ell\left(\operatorname{Pic}\left(X_{\Sigma, L}\right)\right)} \frac{\prod_{v \in S} L_{v}(1, X(T)) L_{v}\left(1, \operatorname{Pic}\left(X_{\Sigma, L}\right)\right)}{\prod_{v \in S} L_{v}\left(1, P_{\Sigma}\right)} \int_{\frac{X_{\Sigma}(K)}{X_{X}}} \omega_{X_{\Sigma}} .
\end{aligned}
$$

D'après le lemme 2.4 et la suite exacte $(4.11)$, on a

$$
\ell\left(P_{\Sigma}\right)=\ell(X(T)) \ell\left(\operatorname{Pic}\left(X_{\Sigma, L}\right)\right)
$$

et

$$
\frac{\prod_{v \in S} L_{v}(1, X(T)) L_{v}\left(1, \operatorname{Pic}\left(X_{\Sigma, L}\right)\right)}{\prod_{v \in S} L_{v}\left(1, P_{\Sigma}\right)}=1
$$

d'où le résultat annoncé, au vu de la définition (4.10) de $\gamma_{H}\left(X_{\Sigma}\right)$. 


\subsubsection{Cas arithmétique}

D'après la relation (3.15) du lemme 3.32, on a

$$
[A(T)]=\frac{\left[H^{1}\left(G, \operatorname{Pic}\left(X_{\Sigma, L}\right)\right)\right]}{[\amalg(T)]} .
$$

De plus, par le théorème d'Ono (théorème 3.41), et la définition (3.12) de $\tau(T)$, on a

$$
\ell(X(T))=\frac{b(T)}{\tau(T)}=\frac{b(T)[\amalg(T)]}{\left[H^{1}(G, X(T))\right]} .
$$

On en déduit, compte tenu du lemme 8.8,

$$
\begin{aligned}
{[A(T)] C_{0} } & =[A(T)] \ell(X(T)) \gamma_{H}\left(X_{\Sigma}\right) \\
& =\frac{b(T)}{\left[H^{1}(G, X(T))\right]}\left[H^{1}\left(G, \operatorname{Pic}\left(X_{\Sigma, L}\right)\right)\right] \gamma_{H}\left(X_{\Sigma}\right) \\
& =\frac{b(T)}{\left[H^{1}(G, X(T))\right]} \beta\left(X_{\Sigma}\right) \gamma_{H}\left(X_{\Sigma}\right) .
\end{aligned}
$$

Dans le cas arithmétique, le terme principal de la fonction zêta des hauteurs en $s=1$ est donc, d'après (8.9),

$$
\begin{aligned}
{\left[H^{1}(G, X(T))\right] \alpha^{*}\left(X_{\Sigma}\right) \frac{1}{\left[H^{1}(G, X(T))\right]} } & \beta\left(X_{\Sigma}\right) \gamma_{H}\left(X_{\Sigma}\right) \\
& =\alpha^{*}\left(X_{\Sigma}\right) \beta\left(X_{\Sigma}\right) \gamma_{H}\left(X_{\Sigma}\right)
\end{aligned}
$$

\subsubsection{Cas fonctionnel}

D'après la relation (3.15) du lemme 3.32, on a

$$
[A(T)][\amalg(T)]=H^{1}\left(G, \operatorname{Pic}\left(X_{\Sigma, L}\right)\right) .
$$

Par le théorème d'Oesterlé (théorème 3.41) et la définition (3.13) de $\tau(T)$, on a

$$
\ell(X(T))=\frac{b(T)}{\tau(T)\left[\mathcal{C}_{T}\right]}=\frac{b(T)[\amalg(T)]}{\left[\mathcal{C}_{T}\right]\left[H^{1}(G, X(T))\right]} .
$$

On en déduit, compte tenu du lemme 8.8,

$$
\begin{aligned}
{[A(T)] C_{0} } & =[A(T)] \ell(X(T)) \gamma_{H}\left(X_{\Sigma}\right) \\
& =\frac{b(T)}{\left[\mathcal{C}_{T}\right]\left[H^{1}(G, X(T))\right]}\left[H^{1}\left(G, \operatorname{Pic}\left(X_{\Sigma, L}\right)\right)\right] \gamma_{H}\left(X_{\Sigma}\right) \\
& =\frac{b(T)}{\left[\mathfrak{C}_{T}\right]\left[H^{1}(G, X(T))\right]} \beta\left(X_{\Sigma}\right) \gamma_{H}\left(X_{\Sigma}\right) .
\end{aligned}
$$


Le terme principal de la fonction zêta des hauteurs en $s=1$ est donc, d'après le lemme 8.7,

$$
\begin{array}{r}
{\left[H^{1}(G, X(T))\right]\left[\mathcal{C}_{T}\right] \frac{1}{b(T)} \alpha^{*}\left(X_{\Sigma}\right) \frac{b(T)}{\left[\mathcal{C}_{T}\right]\left[H^{1}(G, X(T))\right]} \beta\left(X_{\Sigma}\right) \gamma_{H}\left(X_{\Sigma}\right)} \\
=\alpha^{*}\left(X_{\Sigma}\right) \beta\left(X_{\Sigma}\right) \gamma_{H}\left(X_{\Sigma}\right) .
\end{array}
$$

Ceci achève la démonstration du théorème 4.13.

\section{A Appendice : le cas où l'hypothèse 4.8 n'est pas vérifiée}

Dans le cas fonctionnel, nous indiquons à présent comment adapter ce qui précède au cas où certaines places de $v$ ne vérifient pas l'hypothèses 4.8 . Soit $e$ un entier strictement positif divisibles par tous les $e_{v}$.

Ainsi, pour tout $v \in \mathcal{P}_{K}$, pour tout $\varphi \in e \operatorname{PL}(\Sigma)^{G}$ et pour tout $t \in T\left(K_{v}\right)$, $f_{v}\langle\varphi, t\rangle_{\Sigma, v}$ est entier

On peut donc définir un accouplement

$$
\begin{aligned}
\mathfrak{H}_{e, v}: \quad\left(e \operatorname{PL}(\Sigma)^{G}\right)_{\mathbf{C}^{\times}} \times T\left(K_{v}\right) & \longrightarrow \mathbf{C}^{*} \\
(\psi, t) & \longmapsto\langle\psi, t\rangle_{\Sigma, v}^{f_{v}}
\end{aligned}
$$

vérifiant

$$
\forall \varphi \in\left(e \operatorname{PL}(\Sigma)^{G}\right)_{\mathbf{C}}=\operatorname{PL}(\Sigma)_{\mathbf{C}}^{G}, \quad \forall t \in T\left(K_{v}\right), \quad H_{v}(\varphi, t)=\mathfrak{H}_{e, v}\left(q^{\varphi}, t\right) .
$$

Dans toute la suite, on identifiera $\operatorname{PL}(\Sigma)_{\mathbf{C}}^{G}$ à $\mathbf{C}^{\Sigma(1) / G}$ non plus au moyen de la base $\left(D_{\alpha}\right)$, mais de la base $\left(e D_{\alpha}\right)$. On notera $\left(s_{\alpha}^{\prime}\right)$ l'élément de $\mathbf{C}^{\Sigma(1) / G}$ correspondant à un élément de $s$ de $\operatorname{PL}(\Sigma)_{\mathbf{C}}^{G}$ via cette identification. Ainsi on a $s_{\alpha}=e s_{\alpha}^{\prime}$ pour tout $\alpha \in \Sigma(1) / G$.

Soit

$$
P=\sum_{\left(n_{\alpha}\right) \in \mathbf{N}^{\Sigma(1) / G}} a_{\left(n_{\alpha}\right)} \prod z_{\alpha}^{n_{\alpha}}
$$

une série formelle, et $\boldsymbol{z}$ un élément de $\left(\mathbf{C}^{\times}\right)^{\Sigma(1) / G}$ tel que la série définissant $P(\boldsymbol{z})$ converge absolument. Si on voit $\boldsymbol{z}$ comme un élément de $\left(e P_{\Sigma}^{G}\right)_{\mathbf{C}^{\times}}$, on a donc

$$
P(\boldsymbol{z})=\sum_{\left(n_{\alpha}\right) \in \mathbf{N}^{\Sigma(1) / G}} a_{\left(n_{\alpha}\right)}\left\langle\boldsymbol{z}, \frac{1}{e} \sum n_{\alpha} D_{\alpha}^{\vee}\right\rangle
$$


Pour tout $\boldsymbol{z} \in\left(e X(T)^{G}\right) \mathbf{C}^{*}$ tel que la série définissant $P\left(\gamma_{\mathbf{C}^{\times}}(\boldsymbol{z})\right)$ converge absolument, on a donc

$$
P\left(\gamma_{\mathbf{C}^{\times}}(\boldsymbol{z})\right)=\sum_{\left(n_{\alpha}\right) \in \mathbf{N}^{\Sigma(1) / G}} a_{\left(n_{\alpha}\right)}\left\langle\boldsymbol{z}, \frac{1}{e} \sum n_{\alpha} \rho_{\alpha}\right\rangle .
$$

La définition de la compatibilté est modifiée comme suit : pour tout sousgroupe $M$ de $\frac{1}{e}\left(X(T)^{G}\right)^{\vee}$ et tout $\left(n_{\alpha}\right) \in \mathbf{N}^{\Sigma(1) / G}$, le monôme $\prod_{\alpha \in \Sigma(1) / G} z_{\alpha}^{n_{\alpha}}$ est $\operatorname{dit} M$-compatible si on a

$$
\gamma^{\vee}\left(\frac{1}{e} \sum n_{\alpha} D_{\alpha}^{\vee}\right) \in M
$$

autrement dit si on a

$$
\frac{1}{e} \sum n_{\alpha} \rho_{\alpha} \in M
$$

On voit qu'un monôme $f$ est $M$-compatible si et seulement s'il existe un élément $m$ de $M$ vérifiant

$$
\forall \boldsymbol{z} \in\left(e X(T)^{G}\right)_{\mathbf{C}^{*}}, \quad f\left(\gamma_{\mathbf{C}^{\times}}(\boldsymbol{z})\right)=\langle\boldsymbol{z}, m\rangle
$$

soit encore

$$
\forall \boldsymbol{z} \in X(T)_{\mathbf{C}^{\times}}^{G}, \quad f\left(\gamma_{\mathbf{C}^{\times}}\left(\boldsymbol{z}^{e}\right)\right)=\left\langle\boldsymbol{z}^{e}, m\right\rangle .
$$

Par ailleurs pour tout $\alpha \in \Sigma(1) / G$, le monôme $z_{\alpha}^{e d_{\alpha}}$ est $\mathcal{D}_{T}^{0}$-compatible.

On peut vérifier alors que tous les résultats de la sous-section 5.4.3 (à partir du lemme 5.21) et de la sous-section 5.5.2 restent valable, en remplac̀ ;ant dans les énoncés et définitions toutes les occurences de $\mathfrak{H}_{v}$ (respectivement de $\chi_{\boldsymbol{z}}$ pour $\boldsymbol{z} \in X(T)_{\boldsymbol{U}}^{G}$, respectivement de $\gamma_{\boldsymbol{U}}(\boldsymbol{z})$ pour $\left.\boldsymbol{z} \in X(T)_{\boldsymbol{U}}^{G}\right)$ par $\mathfrak{H}_{e, v}$ (respectivement $\chi_{\boldsymbol{z}^{e}}$, respectivement $\gamma_{\boldsymbol{U}}(\boldsymbol{z})$ ) et toutes les occurences des expressions du type «de rayon de convergence supérieur à $q^{a} \gg$ avec $a \in \mathbf{R}$ par «de rayon de convergence supérieur à $q^{\frac{a}{e}} 》$. 
On obtient pout tout $\boldsymbol{s} \in \mathcal{T}\left(\mathbf{R}_{>\frac{1}{e}}^{\Sigma(1) / G}\right)$ et tout $\chi \in\left(T\left(\mathbf{A}_{K}\right) / \mathbf{K}(T)\right)^{*}$

$$
\begin{aligned}
I_{1}(\boldsymbol{s}) & =\int_{X(T)_{U}^{G}}\left[\int_{\left.\frac{T(K)}{T(T)} \mathbf{A}_{K}\right)} H(-\boldsymbol{s}, t) \chi_{\boldsymbol{z}}(t) \omega_{T}(t)\right] d \boldsymbol{z} \\
& =\int_{X(T)_{U}^{G}}\left[\int_{\overline{T(K)} \cap T\left(\mathbf{A}_{K}\right)} H(-\boldsymbol{s}, t) \chi_{\boldsymbol{z}^{e}}(t) \omega_{T}(t)\right] d \boldsymbol{z} \\
& =\int_{X(T)_{U}^{G}}\left(\prod_{\alpha \in \Sigma(1) / G} Z_{K_{\alpha}}\left(\left\langle\boldsymbol{z}^{e}, d_{\alpha} \rho_{\alpha}\right\rangle q^{-d_{\alpha} e s_{\alpha}^{\prime}}\right)\right) \mathfrak{f}\left(\gamma_{\boldsymbol{U}}\left(\boldsymbol{z}^{e}\right) q^{-\boldsymbol{s}}\right) d \boldsymbol{z}
\end{aligned}
$$

et

$$
\begin{aligned}
I_{\chi}(\boldsymbol{s}) & =\int_{X(T)_{U}^{G}} \mathcal{F} H\left(\chi_{\boldsymbol{z}} \chi,-\boldsymbol{s}\right) d \boldsymbol{z} \\
& =\int_{X(T)_{U}^{G}} \mathcal{F} H\left(\chi_{\boldsymbol{z}^{e}} \chi,-\boldsymbol{s}\right) d \boldsymbol{z} \\
& =\int_{X(T)_{U}^{G}}\left(\prod_{\alpha \in \Sigma(1) / G} \mathcal{L}_{K_{\alpha}}\left(\chi_{\alpha},\left\langle\boldsymbol{z}^{e}, d_{\alpha} \rho_{\alpha}\right\rangle q^{-d_{\alpha} e s_{\alpha}^{\prime}}\right)\right) \mathfrak{f}\left(\chi, \gamma_{\boldsymbol{U}}\left(\boldsymbol{z}^{e}\right) q^{-\boldsymbol{s}}\right) d \boldsymbol{z}
\end{aligned}
$$

On peut alors adapter le raisonnement de la sous-section 8.3.2. Pour $I_{\chi}$, le rôle de la suite exacte (7.1) est joué par la suite exacte

$$
0 \longrightarrow\left(\pi\left(e P_{\Sigma}^{G}\right)\right)^{\vee} \stackrel{\pi^{\vee}}{\longrightarrow} \frac{1}{e}\left(P_{\Sigma}^{G}\right)^{\vee} \stackrel{\gamma^{\vee}}{\longrightarrow} \frac{1}{e}\left(X(T)^{G}\right)^{\vee} \longrightarrow 0
$$

et pour $I_{1}$, par la suite exacte

$$
0 \longrightarrow \mathcal{D}_{T_{\mathrm{NS}}}^{0} \stackrel{\pi^{\vee}}{\longrightarrow} \mathcal{D}_{T_{P_{\Sigma}}} \stackrel{\gamma^{\vee}}{\longrightarrow} \mathcal{D}_{T}^{0} \longrightarrow 0 .
$$

Comme dans le cas déjà traité, on en déduit qu'il existe une fonction admissible $g$ de multiplicité supérieure à zéro et vérifiant

$$
g(0)=\log q^{[\Sigma(1) / G]}\left(\prod_{\alpha} d_{\alpha}\right) C_{0}
$$


telle que la fonction

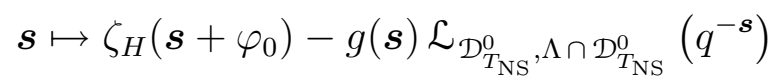

est admissible de multiplicité supérieure à $1-\operatorname{rg}\left(\operatorname{Pic}\left(X_{\Sigma}\right)\right)$.

\section{Références}

[Ar] E. Artin. Über eine neue Art von L-Reihen. Abh. Math. Sem. Univ. Hamburg, 3, 1924. p. 89-108.

[BaTs1] V.V. BATyRev, Y. Tschinkel. Rational points of bounded height on compactifications of anisotropic tori. Int. Math. Res. Notices, 12, 1995. p. 591-635.

[BaTs2] V.V. Batyrev, Y. Tschinkel. Manin's conjecture for toric varieties. J. of Algebraic Geometry, 7, 1998. p. 15-53.

[BaTs3] V.V. Batyrev, Y. Tschinkel. Rational points on some Fano cubic bundles. C. R. Acad. Sci. Paris Sér. I Math., 323, 1996. p. 41-46.

[BaTs4] V.V. BATyrev, Y. Tschinkel. Height zeta functions of toric varieties. J. Math. Sci., 82, 1996. p. 3220-3239.

[BoSp] A. Borel, T.A. Springer. Rationality properties of linear algebraic groups. II. Tôhoku Math. J. (2), 20, 1968. p. 443-497.

[Bki1] N. BouRBAKI. Éléments de mathématiques XXXII : Théories spectrales. Chapitres 1 et 2. Actualités scientifiques et industrielles 1332, Hermann, Paris, 1967.

[Bki2] N. Bourbaki. Éléments de mathématiques XXXVI : Variétés différentielles et analytiques. Fascicule de résultats (Paragraphes 8 à 15). Actualités scientifiques et industrielles 1347, Hermann, Paris, 1971.

[Bo1] D. Bourqui. Fonction zêta des hauteurs des surfaces de Hirzebruch dans le cas fonctionnel. J. of Number Theory, 94, 2002. p. 343-358.

[Bo2] D. Bourqui. Fonction zêta des hauteurs des variétés toriques déployées dans le cas fonctionnel. J. reine angew. Math., 562, 2003. p. 171199.

[Bo3] D. Bourqui. Fonctions zêta des hauteurs des variétés toriques en caractéristique positive. Thèse de doctorat, Grenoble, 2003. Disponible à l'URL http://tel.ccsd.cnrs.fr/tel-00004008

[Bro] T.D. Browning. The Manin conjecture in dimension 2. Preliminary version of lecture notes for the "School and conference on analytic number theory", ICTP, Trieste, 23/04/07-11/05/07, arXiv:0704.1217v1. 
[CLTs] A. Chambert-Loir, Y. Tschinkel. Fonctions zêta des hauteurs des espaces fibrés in Rational points on algebraic varieties, E. Peyre \& Y. Tschinkel, eds. Birkhäuser (2001). p. 71-115.

[CTHaSk] J.-L. Colliot-Thélène, D. Harari, N. Skorobogatov. Compactification équivariante d'un tore (d'après Brylinski et Künnemann) Expo. Math., 23, no. 2, 2005. p. 161-170.

[CTSa] J.-L. Colliot-Thélène, J.-J. SAnsuc. La R-équivalence sur les tores. Ann. scient. Ec. Norm. Sup., 10, 1977. p. 175-230.

[CTSu] J.-L. Colliot-ThÉlène, V. Suresh. Quelques questions d'approximation faible pour les tores algébriques. À paraître aux Annales de l'Institut Fourier.

[Dr] P.K.J. DraxL. L-Funktionen K-algebraischer Tori. J. of Number Theory, 3, 1971. p. 444-467.

[FrMaTs] J. Franke, Y. Manin, Y. Tschinkel. Rational points of bounded height on Fano varieties. Invent. Math., 95, 1989. p. 421-435.

[Fu] W. Fulton. Introduction to toric varieties. Annals of Mathematics Studies 31, Princeton University Press, 1993.

[Ha] H. HASSE. Number theory. Grundlehren der mathematischen Wissenschaften 229, Springer, 1980.

[HoNa] G. Hochschild, T. Nakayama. Cohomology in class field theory. Ann. of Math., 55, 1952. p. 348-366.

[LaYe] K.F. LAI, K.M. Yeung. Rational points in flag varieties over function fields. J. of Number Theory, 95, 2002. p. 142-149.

[Na] T. Nakayama. Cohomology of class field theory and tensor product modules I. Ann. of Math., 65, 1957. p. 265-267.

[Od] T. OdA. Convex bodies and algebraic geometry. Ergebnisse der Mathematik und ihrer Grenzgebiete 15, Springer-Verlag, 1988.

[Oe] J. Oesterlé. Nombre de Tamagawa et groupes unipotents en caractéristique $p$. Invent. Math., 78, 1984. p. 13-88.

[On1] T. Ono. On some arithmetic properties of linear algebraic groups. Ann. of Math., 70, 1959. p. 266-290.

[On2] T. Ono. Arithmetic of algebraic tori. Ann. of Math., 74, 1961. p. 101-139.

[On3] T. Ono. On the Tamagawa number of algebraic tori. Ann. of. Math., 78, 1963. p. 47-73.

[Pe1] E. Peyre. Hauteurs et mesures de Tamagawa sur les variétés de Fano. Duke Math. Journal, 79, 1995. p. 101-218. 
[Pe2] E. PeYRE. Torseurs universels et méthode du cercle in Rational points on algebraic varieties. Progr. Math. 79, Birkhaüser, 2001. p. 221-274.

[Pe3] E. Peyre. Points de hauteur bornée sur les variétés de drapeaux en caractéristique finie. Prépublication, math.NT/0303067.

[Pe4] E. Peyre. Etude asymptotique des points de hauteur bornée. Notes de l'école d'été sur les variétés toriques, Grenoble, juin 2000.

[Pe5] E. Peyre. Points de hauteur bornée et géométrie des variétés, d'après Y. Manin et al. Séminaire Bourbaki 891, juin 2001.

[Pe6] E. Peyre. Points de hauteur bornée, topologie adélique et mesure de Tamagawa. J. Th. Nombres de Bordeaux, 15, 2003. p. 319-349.

[PlRa] V. Platonov, A. Rapinchuk. Algebraic groups and number theory. Pure and Applied Mathematics, 139, Academic Press, Boston, 1994.

[Ra] H. Rademacher. On the Phragmén-Lindelöf theorem and some applications. Math. Z., 72, 1959. p. 192-204.

[Sa] P. SAlBerger. Tamagawa measure on universal torsors and points of bounded height on Fano varieties. Astérisque, 251, 1998. p. 91-258.

[Se1] J.-P. SERRE. Corps locaux. Hermann, 1962.

[Se2] J.-P. SERRE. Lectures on the Mordell-Weil theorem, translated and edited by M. Brown. Aspect of Mathematics, Vieweg, 1989.

[Vo] V.E. VoskresenskiI. Projective invariant Demazure models. Math. USSR Izv., 20, no. 2, 1983. p. 189-202.

[Vo] V.E. VoskresenskiI. Invariants birationnels des tores algébriques (en russe). Uspehi Mat. Nauk, 30, 1975. p. 207-208.

[Wa] D. WAn. Heights and zeta functions in function fields in The arithmetic of function fields, D. Goss, D.R. Hayes \& M.I. Rosen, eds. Mathematical Research Institute Publications, Ohio State University, de Gruyter, 1992. p. 455-463.

[We1] A. WeIL. Basic number theory. Springer Verlag, 1967.

[We2] A. WeIL. Adeles and algebraic groups. Progress in mathematics 23, Birkhaüser, 1982. 


\section{Index des notations}

$\mathcal{O}_{v}, 12$

$A(T), 48$

$C_{K}^{1}, 40$

$C_{V, H}^{*}, 20$

$C_{K}, 23$

$C_{V, H}, 20$

$H_{\mathcal{L}}, 19$

$K_{v}, 12$

$L(s, M), 15$

$L_{E}(\chi, s), 80$

$L_{v}(s, M), 15$

$N^{\vee}, 11$

$N_{G}, 36$

$T\left(C_{K}\right), 37$

$T\left(\mathcal{O}_{v}\right), 23$

$T\left(\mathbf{A}_{K}\right), 23$

$T\left(\mathbf{A}_{K}\right)^{1}, 28$

$T\left(\mathbf{A}_{K}\right)_{S}^{1}, 28$

$T\left(\mathbf{A}_{K}\right)^{S}, 48$

$T\left(\mathbf{A}_{K}\right)_{S}, 23$

$T\left(C_{K}\right)^{1}, 40$

$X(T), 20$

$X(T)_{\mathbf{R}, \infty}, 94$

$Z_{K}, 14$

$\mathrm{e}_{T}, 35$

$\mathcal{D}_{T}, 35$

$\mathcal{D}_{T}^{0}, 83$

$\mathbf{F}_{K}, 12$

$\mathbf{G}_{m}\left(\mathbf{A}_{K}\right), 22$

$\mathbf{G}_{m}\left(\mathbf{A}_{K}\right)^{1}, 25$

$\mathbf{K}(T), 24$

$\mathbf{K}\left(\mathbf{G}_{m}\right), 22$

$\mathcal{K}_{T}, 54$

$K_{\alpha}, 63$

$\mathcal{O}_{S}, 13$

$\operatorname{PL}(\Sigma), 62$

$P_{\Sigma}, 62$

$\operatorname{Res}_{K_{0} / K} \mathbf{G}_{m}, 21$
$\Sigma(1), 61$

|. $\left.\right|_{v}, 13$

$\alpha^{*}(V), 19$

$\beta(V), 20$

Ш $(T), 38$

$\chi_{y}, 81$

$\chi_{\boldsymbol{z}}, 82$

$\mathcal{C}_{K}, 12$

$d_{\alpha}, 83$

$\operatorname{deg}_{T}, 26$

$\operatorname{deg}_{K, v}, 25$

$\operatorname{deg}_{K}, 25$

$\operatorname{deg}_{T, L, \mathcal{V}}, \mathcal{V}$ archimédienne, 31

$\operatorname{deg}_{T, L, \mathcal{V}}, \mathcal{V}$ finie, 30

$\operatorname{deg}_{T, L}$ (cas arithmétique), 29

$\operatorname{deg}_{T, L}$ (cas fonctionnel), 29

$\operatorname{deg}_{T, v}, 27$

$\operatorname{disc}(K), 12$

$d_{K}, 12$

$\mathcal{L}_{E}(\chi,),$.

$\mathcal{L}_{N, A, a}, 129$

$\mathcal{L}_{N, A}, 130$

$\mathcal{U}_{T}, 109$

$\ell(M), 15$

$\mathcal{F} H(-\varphi), 79$

$\mathfrak{H}_{S}(., t), 99$

$\mathfrak{H}_{v}, 70$

$\mathfrak{Q}(\chi,),$.

$\mathfrak{f}(),$.

$\mathfrak{f}(\chi,),$.

$\mathfrak{f}_{v}(\chi,),$.

$G_{\alpha}, 63$

$\gamma^{*}, 109$

$i_{\alpha}, 63$

intrel, 60

$\omega_{T}, 59$

$\omega_{T}^{1}, 59$

$\omega_{V}, 19$ 
$\mathcal{P}_{K, \infty}, 12$

$\mathcal{P}_{K, f}, 12$

$\mathcal{P}_{K}, 12$

$q_{K}, 12$

$\rho_{\alpha}, 63$

$\Sigma(1) / G, 63$

$\sigma(1), 61$

$\tau(T), 60$

$\tau_{H}(V), 20$

$\mathcal{T}(U), 12$

$\boldsymbol{U}, 76$

$\varphi_{0}, 71$

$\zeta_{H}, 71$

$\zeta_{K}, 13$

$c_{K, d}, 19$

$d x_{v}, 13$

$f(\chi,),$.

$f_{K}$, où $f$ est un morphisme de tores algébriques, 22

$f_{v}, 13$

$f_{v}$, où $f$ est un morphisme de tores algébriques, 24

$f_{\mathbf{A}_{K}}$, où $f$ est un morphisme de tores algébriques, 24

$f_{\infty}(\chi,),$.

$g_{K}, 12$

$i_{T, v}, 27$

$q_{v}, 13$

$k_{v}, 12$ 


\section{Index des définitions}

$G$-éventail, 63

$G$-module flasque, 46

$\Xi$-fonction, 127

éventail, 61

cône dual, 60

cône polyédral rationnel, 61

cas arithmétique, 12

cas fonctionnel, 12

corps de fonctions, 12

corps des constantes, 12

fonction admissible de multiplicité

supérieure à $-r, 133$

hauteur d'Arakelov, 18

métrique $v$-adique, 17

métrique adélique, 17

métrique adélique standard, 18

monôme, 95

morphisme «type à l'infini», 94

résolution flasque, 46

rayons d'un éventail, 61

série formelle, 95

tore algébrique, 20

tore algébrique quasi-déployé, 21 
I.R.M.A.R

Campus de Beaulieu

35042 Rennes cedex

France

david.bourqui@univ-rennes 1.fr 